\title{
HIGH-ENERGY PHOTOPRODUCTION OF RHO AND PHI VECTOR MESONS
}

\section{DO NOT MICROFILM COVER}

BY

PAUL HAYWARD CALLAHAN

B.A., State University of New York at Geneseo, 1976

M.S., University of Illinois, 1978

\section{THESIS}

Submitted in partial fulfillment of the requirements

for the degree of Doctor of Philosophy in Physics in the Graduate college of the

University of Illinois at Urbana-Champaign, 1983

Urbana, Illinois

\section{MASTER}

PACS $13.6 \emptyset$. Le 


\section{DISCLAIMER}

This report was prepared as an account of work sponsored by an agency of the United States Government. Neither the United States Government nor any agency Thereof, nor any of their employees, makes any warranty, express or implied, or assumes any legal liability or responsibility for the accuracy, completeness, or usefulness of any information, apparatus, product, or process disclosed, or represents that its use would not infringe privately owned rights. Reference herein to any specific commercial product, process, or service by trade name, trademark, manufacturer, or otherwise does not necessarily constitute or imply its endorsement, recommendation, or favoring by the United States Government or any agency thereof. The views and opinions of authors expressed herein do not necessarily state or reflect those of the United States Government or any agency thereof. 


\section{DISCLAIMER}

Portions of this document may be illegible in electronic image products. Images are produced from the best available original document. 


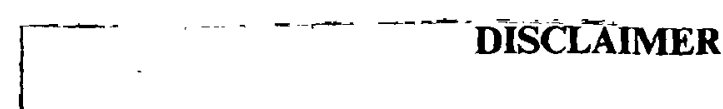

This report was prepared as an account of work sponsored by an agency of the United States Government. Neither the United States Government nor any agency thereof, nor any of their employees, makes any warranty, express or implied, or assumes any legal liability or responsibility for the accuracy, completeness, or usefulness of any information, apparatus, product, or process disclosed, or represents that its use would not infringe privately owned rights. Reference herein to any specific commercial product, process, or service by trade name, trademark, manufacturer, or otherwise does not necessarily constitute or imply its endorsement, recommendation, or favoring by the United States Government or any agency thereof. The views and opinions of authors expressed herein do not necessarily state or reflect those of the -Inited States Government or any agency thereof.

$$
\begin{aligned}
& \text { HIGH-ENERGY PHOTOPRODUCTION OF } \\
& \text { RHO AND PHI VECTOR MESONS } \\
& \text { DDE/ER/OL/ } / 9.5-474
\end{aligned}
$$$$
\text { DOE/ER/01195--474 }
$$$$
\text { DE83 } 007418
$$

BY

\author{
PAUL HAYWARD CALLAHAN \\ B.A., State University of New York at Geneseo, 1976 \\ M.S., University of Illinois, 1978
}

\title{
NOTICE
}

PORTIONS OF THIS REPORT ARE ILLEGIBLE. It has been reproduced from the best available ability.

\section{THESIS}

Submitted in partial fulfillment of the requirements for the degree of Doctor of Philosophy in Physics

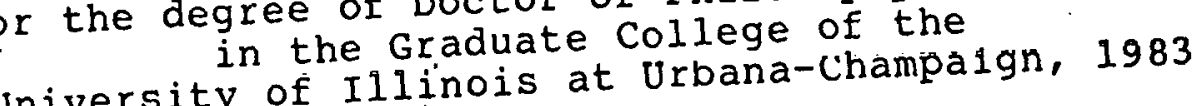

Urbana, Illinois

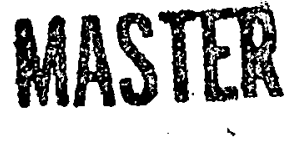

PACS 13.60. Le

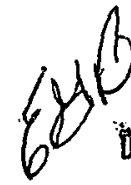




\section{HIGH ENERGY PHOTOPRODUCTJON OF RHO AND PHI VECTOR MESONS \\ Paul Hayward Cállahan, Ph.D. Department of Physics \\ University of Illinois at Urbana-Champaign, 1983}

We have studied the photoproduction of $\rho$ and $\phi$ vector mesons from hydrogen in the Fermilab broad band neutral beam. Forward going two particle final states were detected in a multiparticle spectrometer consisting of two analyzing magnets, a multiwire-proportional-chamber tracking system and a particle identification system. Recoil protons and target fragments were observed in a recoil detector which surrounded the target. The total elastic cross-sections were measured to be independent of energy at the $1 \emptyset \%$ level from 35 to $225 \mathrm{GeV}$ at $10.6 \mu \mathrm{b}$ for the $\rho$ and from 35 to $165 \mathrm{GeV}$ at $0.64 \mu \mathrm{b}$ for the $\phi$. The elastic differential cross-sections were also measured. Approximately 20\% of the diffractive $\rho$ and $\phi$ events were found to be inelastic from an analysis of the recoil detector. The $t$-dependence of the fraction of diffractive events which are inelastic for both the $\phi$ and the $\psi$ are consistent with a universal function determined from the $\rho$ data. 


\section{ACKNOWLEDGMENTS}

The successful completion of an elementary particle physics experiment requires the time and efforts of many people. This acknowledgment is an insufficient "thank you" for the substantial contributions of so many people to the completion of this thesis.

I would like to thank the Accelerator Division personnel at Fermi National Accelerator Laboratory for making the lab one of the finest research facilities in the world. Thanks also go to the Proton Department at Fermilab for their help in making this experiment a successful one.

I am deeply indebted to my hard working colleagues from the Fermilab Phisics Department: Morris Binkley, Joel Butler, John Cumalat, Irwin Gaines, Dave Harding and John Peoples. A special' thank you goes to Mike Gormley for his concern and counsel during hard times. In simplest terms, this thesis would not exist without the many contributions from these people. I cannot satisfactorily express my gratitude to them.

I am equally indebted to my colleagues from the University of Illinois at Urbana-Champaign. Al Wattenberg is a strong leader and positive influence and I was glad, that I had the opportunity to work with him on this experiment. A special salute goes to my co-conspirator and fellow graduate student, Chris Olszewski, for his hard work and many hours of good company. I hope our paths will again cross in the 
future.

Although not directly associated with this experiment, Jim Wiss was always willing to discuss physics problems with me and I thank him for the many "Jim Wiss 5-minute lectures" he gave me during my graduate career.

There is one acknowledgment that stands out among all others. That acknowledgment is for my advisor of the past four years, Gary Gladding. A graduate student's career can be a very positive or a very negative experience depenaing on the quality of the student's relationship with his thesis advisor. Gary's exacting tutelage and active involvement with my work made my graduate school experience one of the best. I hope that the quality of the work in this thesis reflects favorably on him. If it does not, I alone bear the responsibility.

I want to thank Len Seward for the many hours of work he contributed to designing and building equipment for this experiment and for teaching me the tricks of the trade.

Dick Brown, Jerry Wray and Dave Lesny deserve rounds of applause for making the DEC-10 computng facility the best user-oriented timesharing system I have encountered anywnere. Their advice and willing assistance made analyzing millions of events a manageable and even enjoyable task.

I thank Dorothy Schoeps and Wynemia wood for making the many travel arrangements necessary throughout the course of this experiment and for creating a pleasant and helpful 
working environment in the high energy physics office.

One final and very special acknowledgment is for. my lovely, loving wife, Celia. She typed and edited this entire thesis and I give her my heartfelt thanks for her many hours of hard work. This thesis, like my life, was started alone and completed with my wife.

This research was supported under U.S. DOE contract number DE-ACø2-76ERO1195. 
TABLE OF CONTENTS

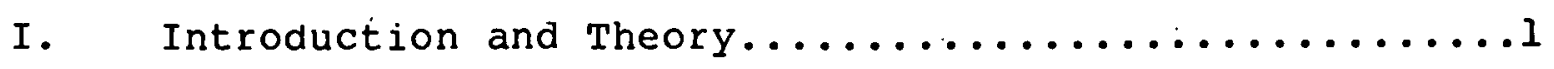

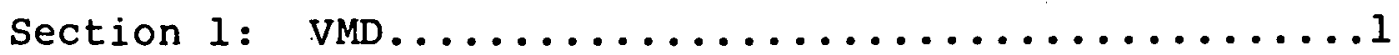

Section 2: Quark Model........................ 6

Section 3: Diffractive Scattering and Factorization.10

Section 4: High Energy Photoproduction of Vector

Mesons.......................11

II. The Experimental Apparatus..................12

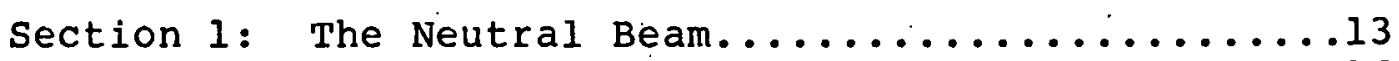

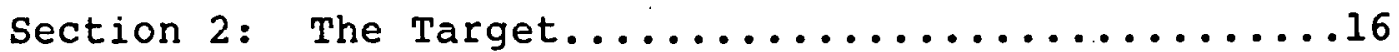

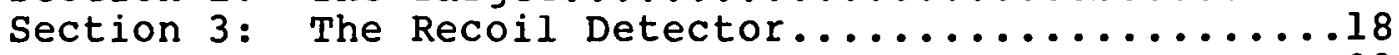

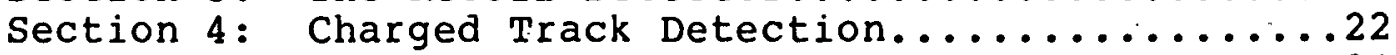

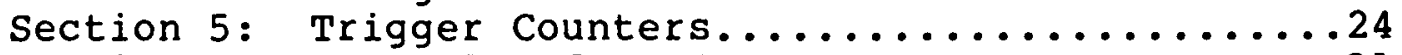

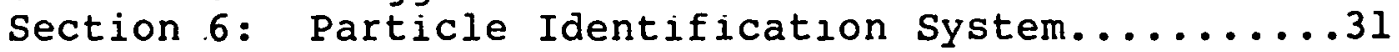

Section 7: The Trigger....................42

Section 8: The Data Aquisition System...............

III. Reconstruction and Edit for Diftractively Produced 2-Track Final states........................ 53

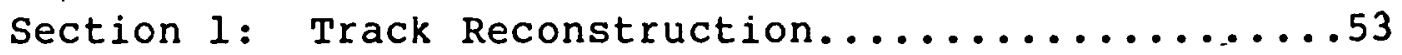

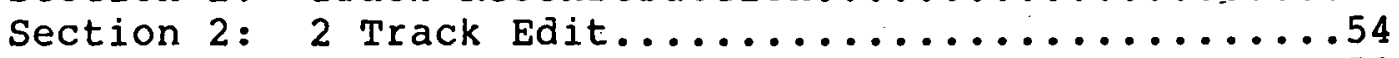

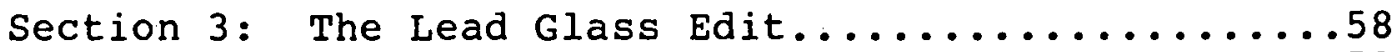

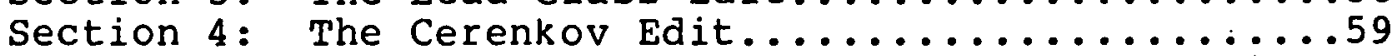

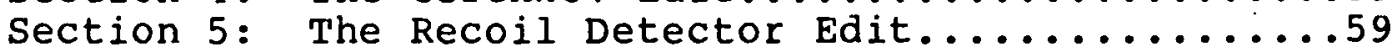

IV. Measurement of the Hadron Calorimeter Threshold.....60

V. Analysis of the Recoil Detector..................

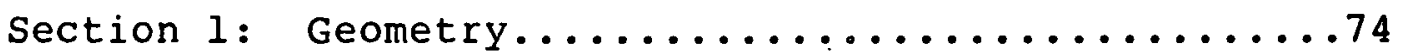

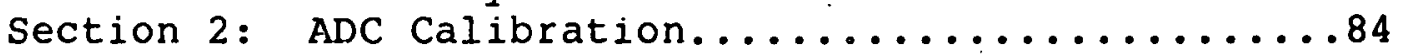

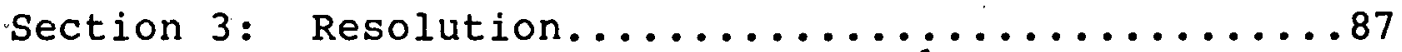

Section 4: Recoil Analysis of the $K_{L}^{0}$ Data.........93

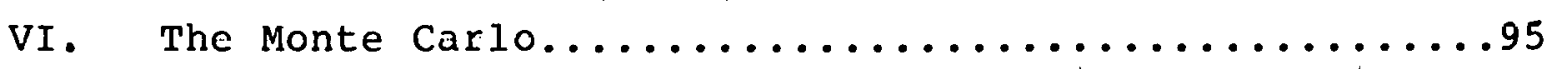


vii

VII. $p$ Analysis.............................

Section 1: Calorimeter Acceptance for the $\rho \ldots \ldots .107$ Section 2: The Energy Dependence of the $\rho$ Cross

Section..........................

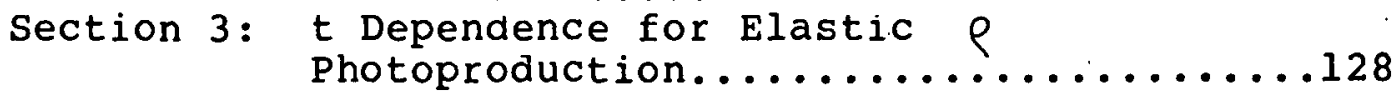

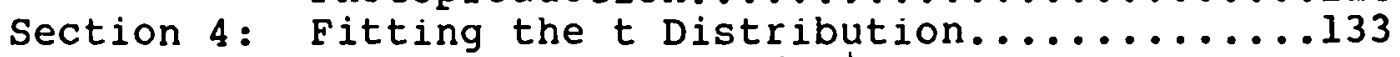

Section 5: Measurement of $\mathrm{d} \sigma /\left.\mathrm{d} t\right|_{t=0} \ldots \ldots \ldots \ldots \ldots \ldots \ldots \ldots \ldots .139$

Section 6: Diffractive Inelastic $\rho$ Production.....146

VIII. $\phi$ analysis............................

Section 1: Calorimeter Acceptance for the $\phi \ldots . . .155$

Section 2: The Energy Dependence of the $\phi$ Cross

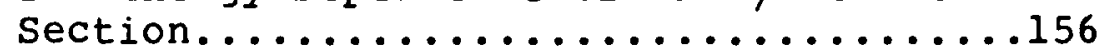

Section 3: $t$ Dependence for Elastic $\phi$

Photoproduction..................164

Section $4:$ Measurement of $\mathrm{d} \sigma /\left.\mathrm{dt}\right|_{t=0} \ldots \ldots \ldots \ldots \ldots \ldots 165$

Section 5: Diffractive Inelastic $\phi$ production.....172

Ix. $\psi$ Analysis............................. $\ldots \ldots$

Section 1: Energy Dependence of $\psi$ Photoproduction.178 Section 2: $t$ Dependence for Elastic $\psi$

Photoproduction.....................

Section 3: Diffractive Inelastic $\psi$ Production.....180

X. Summary and Conclusions................... 186

Section 1: The Energy Dependence of the elastic $\rho$

and $\phi$ Photoproduction Cross Sections...186

Section 2: The $t$ Dependence of the Elastic $\rho, \phi$ and

$\psi$ Photoproduction Cross Sections.......187

Section 3: Diffractive Inelastic $\rho, \phi$ and $\psi$

Photoproduction...................189

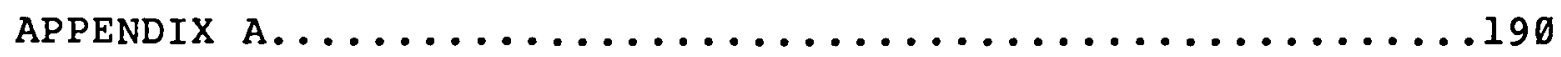

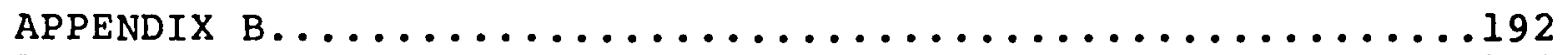

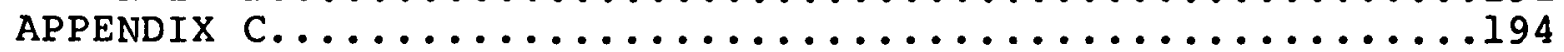

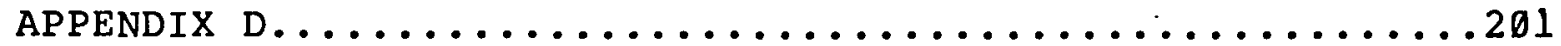

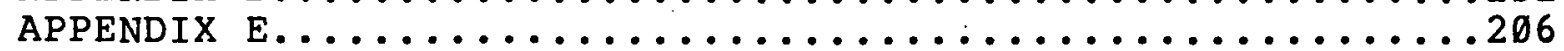

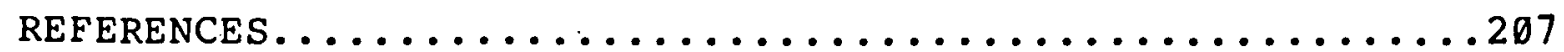

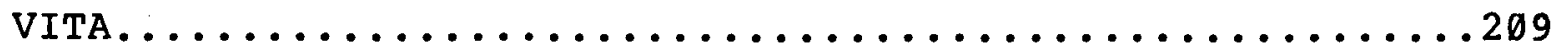




\section{Chapter I}

\section{Introduction and Theory}

The interactions of high energy photons are very similar to hadronic interactions. As far back as 1964, the first systematic study of $\rho$ meson photoproduction by the Cambridge bubble chamber group [1] showed that the cross section had a diffractive character. Above the resonance region, the cross section was approximately independent of energy and had an exponential dependence on the momentum transfer. The only difference between photon induced reactions and hadronic reactions is scale. The photoproduction cross sections are on the order $\sim a$ smaller. Figure 1.1 shows a comparison of the total photon cross section with the average of the $\pi^{+} p$ and $\pi^{-} \rho$ cross sections divided by 200 . The similarity in structure is compelling.

Section 1: VMD

The hadronic interactions of photons are naturally accomodated in the hadron dominance (HD) picture of the photon [2]. Since the photon interacts with hadrons it possesses a hadronic self-energy allowing it to fluctuate with a probability on the order of $\sim \alpha$ into virtual hadronic states. If the photon encounters a target nucleon while in a virtual hadronic state, the scattering will be hadronic in character. The added assumption that these fluctuations are dominated by states with $M_{\gamma}^{2}=M_{V}^{2}$, where $V$ denotes the well defined 


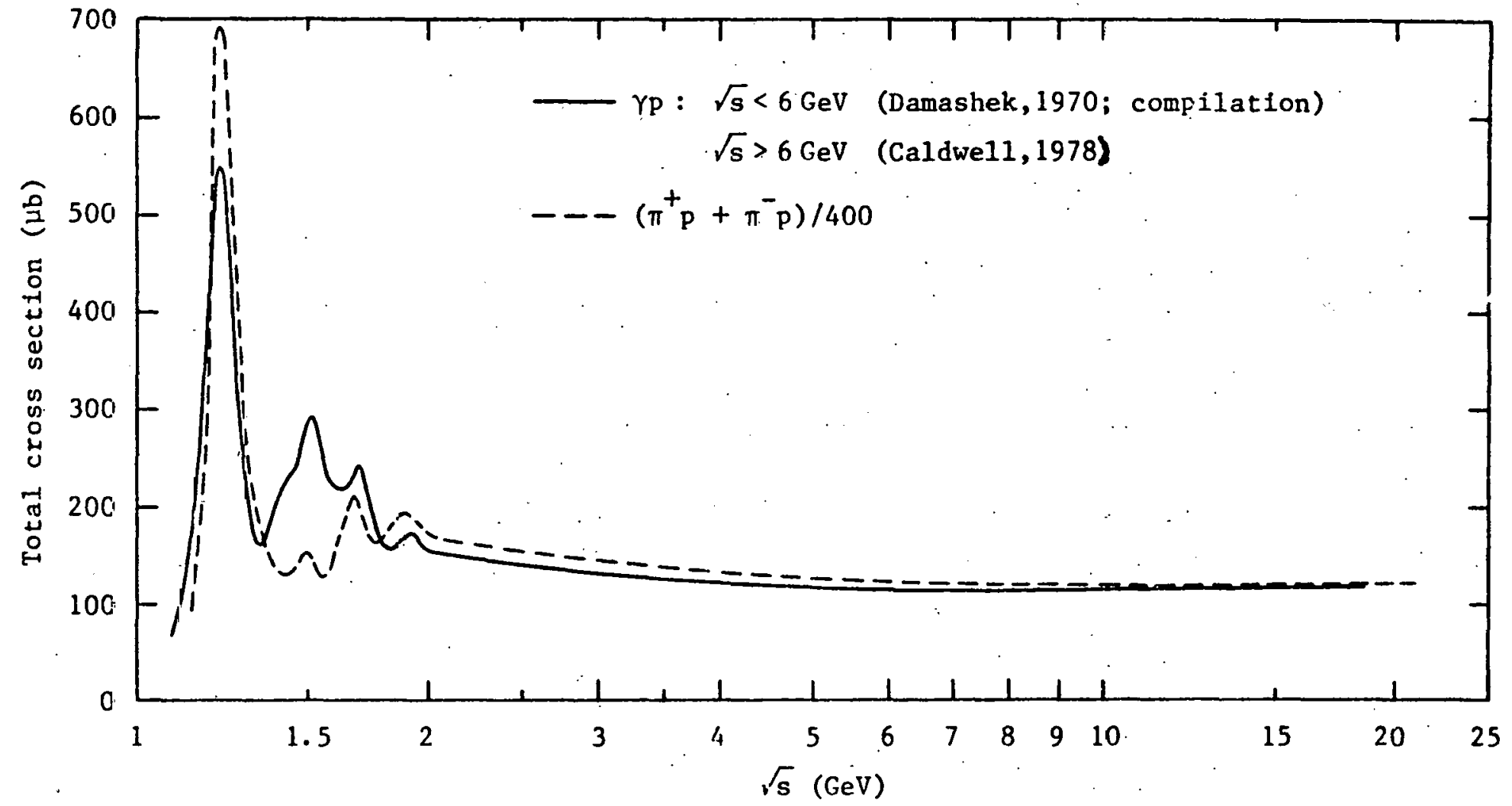

Figure 1.1 
hadronic states with the same quantum numbers as the photon, the vector mesons, is known as the vector. meson dominance mode 1 (VMD) $[3,4,5,6,7]$. This assummption is expressed in the current-field identity relating the electromagnetic current to the vector meson fields:

$f_{\mu}^{e \cdot m}(x)=-\left\{\frac{M_{\rho}^{2}}{2 \gamma_{\rho}} \rho_{\mu}(x)+\frac{M_{\omega}^{2}}{2 \gamma_{\omega}} \omega_{\mu}(x)+\frac{M_{\phi}^{2}}{2 \gamma_{\phi}} \phi_{\mu}(x)+\frac{M_{\psi}^{2}}{2 \gamma_{\psi}} \psi_{\mu}(x)\right\}$

The $\gamma_{V}$ 's are the coupling constants representing the strength of the photon-vector meson coupling. The coupling constants are assumed to be independent of energy and momentum transfer. All such dependence comes from the vector meson propagators. Equation (1.1) is the essence of VMD theory. It relates the matrix elements for photon and vector meson induced reactions:

$$
T(\gamma A \rightarrow B)=e \cdot \sum_{V} \frac{M_{V}^{2}}{2 \gamma_{V}}\left(M_{V}^{2}-Q^{2}\right)^{-1} \cdot T(V A \rightarrow B)
$$

In terms of a Feynman diagram, this can be viewed as:

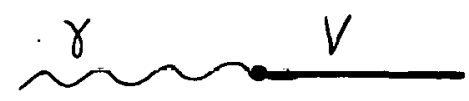


with coupling:

$$
\frac{e M_{V}^{2}}{2 \gamma_{V}} \cdot g \mu v
$$

The diffractive photoproduction amplitude is e/2 $\gamma_{V}$ times the elastic scattering amplitude of the vector meson. The differential cross sections are therefore related by:

$$
\frac{d \sigma}{d t}(\gamma p \rightarrow V P)=\frac{e^{2}}{4 \gamma_{V}^{2}} \cdot \frac{p_{V}^{*^{2}}}{p_{\gamma}^{*^{2}}}: \frac{d \sigma}{d t}(V P \rightarrow V P)
$$

$$
\text { where } \mathrm{p}^{*}=\text { C.M. morientum }
$$

The optical theorem,

$$
\begin{gathered}
\left.\frac{d \sigma}{d t}(A B \rightarrow A B)\right|_{t=0}=\frac{\left(1+n^{2}\right)}{16 \pi} \cdot \sigma^{2}(A B) \\
\text { where } n=\operatorname{Real}(T) / \operatorname{Im}(T),
\end{gathered}
$$

relates the elastic cross section at zero momentum transfer to the square of the total cross section. Together, the optical theorem and VMD allow the measurement of the total meson-nucleon cross section via a photoproduction experiment:

$$
\left.\frac{d \sigma}{d t}(\gamma p \rightarrow V P)\right|_{t=0}=\frac{e^{2}}{4 \gamma_{V}^{2}} \cdot \frac{p_{V}^{*^{2}}}{p_{\gamma}^{*^{2}}} \cdot \frac{\left(1+n^{2}\right)}{16 \pi} \cdot \sigma^{2}(V P)
$$


The progenitor of the experiment analyzed in this thesis was the first experiment to show that the $\psi(3.095)$ was a hadron [8] from just such a measurement of the psi-nucleon total cross section.

If the VMD coupling constants are universal, then the strength of the couplings can be measured directly from the leptonic decay of the vector mesons:

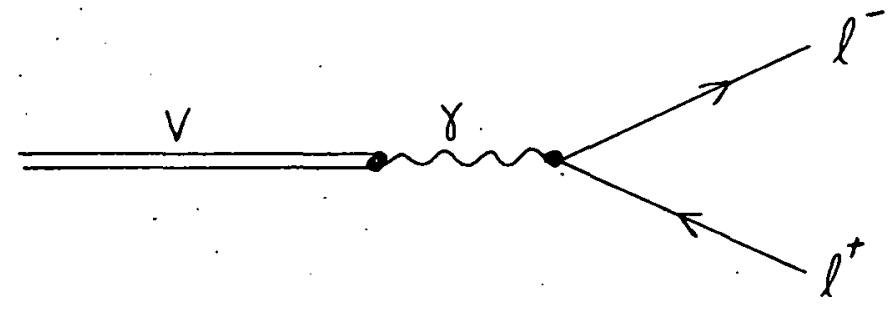

The partial decay width into a two lepton final state, $\Gamma_{l^{+}}^{+} l^{-}$, can be computed exactiy [5]:

$$
\Gamma_{l^{+} l^{-}}\left(V \rightarrow l^{+} l^{-}\right)=\frac{\alpha^{2}}{12}\left(\frac{4 \pi}{\gamma_{V}^{2}}\right) M_{V}\left(1-\frac{4 M_{l}^{2}}{M_{V}^{2}}\right)^{1 / 2}\left(1+\frac{2 M_{l}^{2}}{M_{V}^{2}}\right)
$$

The Leptonic decay widths have been measured in $e^{t} e^{-}$colliding beam experiments [9]. The resulting values for the $\gamma_{V}$ 's, when corrected for tinite width effects [1ø] are listed on the following page. 


$$
\begin{array}{ll} 
& \multicolumn{1}{c}{\gamma_{v}^{2} / 4 \pi} \\
\rho(770) & .64+/-.1 \\
\omega(783) & 4.6+/-.5 \\
\phi(1020) & 2.83+/-.2 \\
\psi(3095) & 2.9+/-.4
\end{array}
$$

The validity of the idea that the hadronic interactions of the photon are dominated by the vector meson resonances, as expressed in VMD theory, is beautifully confirmed in figure 1.2 where the invariant mass distributions for photoproduced $\pi^{+} \pi^{-}, \pi^{+} \pi^{-} \pi^{0}, \mathrm{~K}^{+} \mathrm{K}^{-}$, and $\mu^{+} \mu^{-}$final states from this experiment are shown.

Section 2: Quark Model

Another description of the hadronic interactions of photons within the HD picture is obtained by incorporating the quark-constituent model of the hadrons. The photon undergoes virtual hadronic fluctuations into quark-antiquark pairs, to which the photon is electromagnetically coupled according to the quark charges. This is exactly analogous to the well known virtual fluctuations into $e^{+} e^{-}$pairs. The vector mesons have the following quark content:

$$
\begin{aligned}
& \rho^{0}=1 / \sqrt{2}(u u-d d) \\
& \omega=1 / \sqrt{2}(u u+d d) \\
& \phi=s s \\
& \psi=c c
\end{aligned}
$$




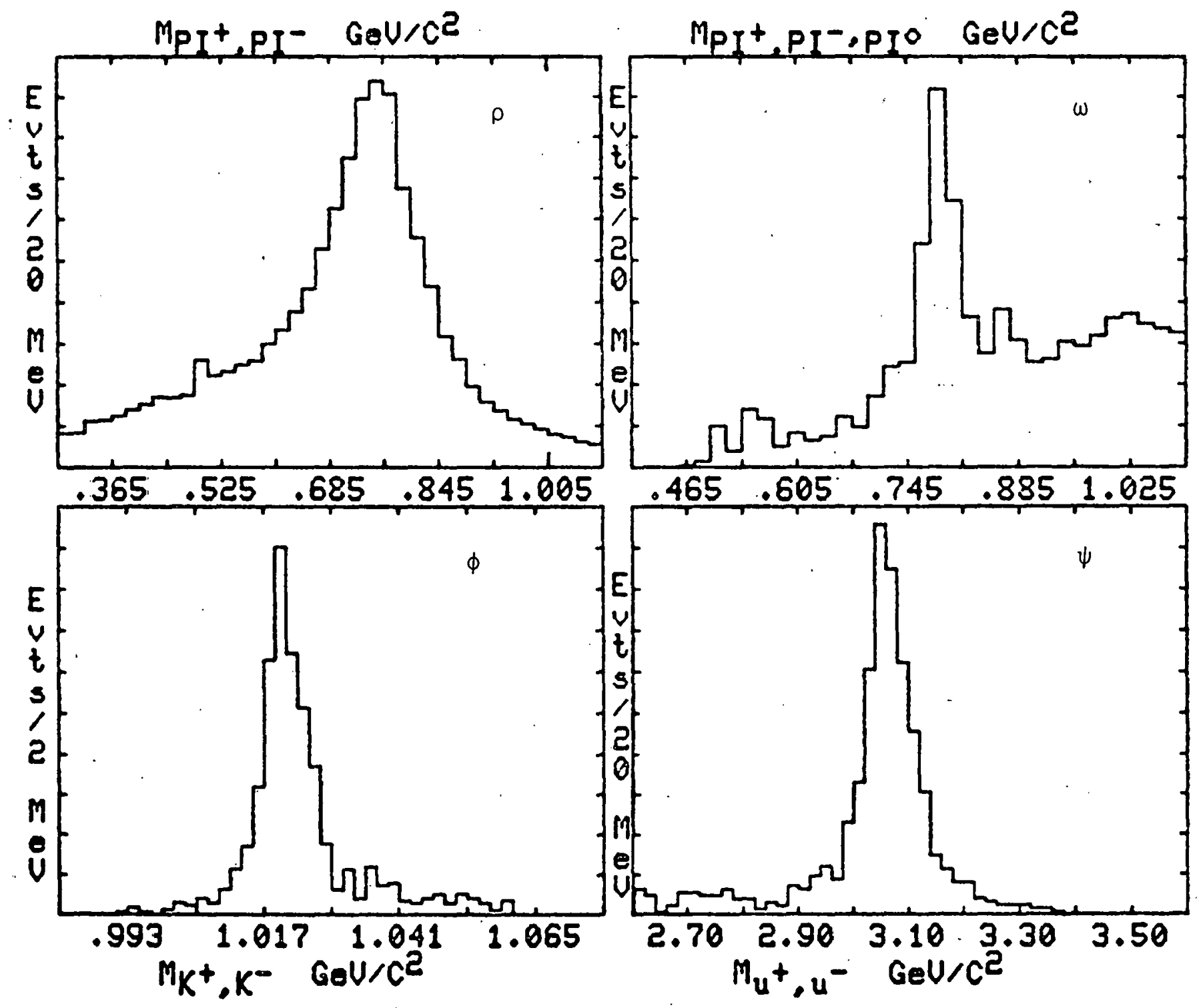

Figure 1.2 
where $u, d, s$, and $c$ are the up, down, strange and charmed quarks, respectively. The quark charges are $u=2 e / 3$, $\mathrm{d}=-\mathrm{e} / 3, \mathrm{~s}=-\mathrm{e} / 3$, and $\mathrm{c}=2 \mathrm{e} / 3$. Comparing with equation (1.1) we see that the simple quark model predicts the ratios of the VMD coupling constants:

$$
\gamma_{\rho}^{-2}: \gamma_{\omega}^{-2}: \gamma_{\phi}^{-2}: \gamma_{\psi}^{-2}=9: 1: 2: 8
$$

Using the measured values for $\gamma_{v}$ from page $(6)$ obtains

$$
\gamma_{\rho}^{-2}: \gamma_{\omega}^{-2}: \gamma_{\phi}^{-2}: \gamma_{\psi}^{-2}=9: 1.25: 2.04: 2.0
$$

The simple quark model prediction agrees very well for the $\rho, \omega$, and $\phi$.

The simple quark model is carried further by assuming the additivity of quark-quark scattering amplitudes, i.e. that scattering from a pion is equivalent to the sum of scattering off of each quark within the pion. In the limit of su(3) symmetry, the $u, d$, and s quarks would all have the same mass. We know that $M_{\omega} \approx M_{\rho}$ but that the strange quarks in the $\phi$ are much heavier. To account for the symmetry breaking of the strange quark, a model was proposed [11] where the quark scattering amplitudes are broken into three independent amplitudes $\mathrm{P}, \mathrm{S}$, and $\mathrm{A}$ representing a non-strange, a strange, and an annihilation amplitude, respectively,

$$
\begin{aligned}
P & =(u d)=(d u)=(\bar{u} d)=(\bar{d} u)=(u u)=(d d) \\
P-S & =(s u)=(s d)=(\bar{s} u)=(\bar{s} d) \\
p+A & =(u \bar{u})=(d \bar{d})
\end{aligned}
$$


where (ud) is the amplitude for an incident u quark to scatter from a d quark, etc. For the scattering of a $\phi$ meson from a proton, for example, we obtain:

$$
\begin{aligned}
(\phi P) & =2(s u)+(s d)+2(\overline{s u})+(\bar{s} d) \\
& =6(P-s)
\end{aligned}
$$

Carrying out identical manipulations for the ( $\rho \mathrm{P}),(\omega \mathrm{P})$, $\left(K^{+} P\right),\left(K^{-} P\right),\left(\pi^{+} P\right)$, and $\left(\pi^{-} P\right)$ scattering amplitudes obtains the predictions of the additive quark model (AQM) that:

$$
\begin{aligned}
& \sigma(\rho P)=\sigma(\omega P)=1 / 2\left[\sigma\left(\pi^{+} P\right)+\sigma\left(\pi^{-} P\right)\right] \\
& \sigma(\phi P)=\sigma\left(K^{+} P\right)+\sigma\left(K^{-} P\right)-\sigma\left(\pi^{-} P\right)
\end{aligned}
$$

The pseudo-scalar meson scattering cross sections can therefore be used with equation [5] to predict vector meson cross sections. The relative normalization between the photoproduction cross sections for vector mesons and pseudo-scalar meson cross sections can then be taken as either a test of the additive quark model using $\gamma_{y}$ from colliding beam measurements, or a measurement of $\gamma_{v}$. assuming quark additivity. 
Section 3: Diffractive Scattering and Factorization

If we assume that elastic vector meson scattering is mediated by Pomeron exchange:

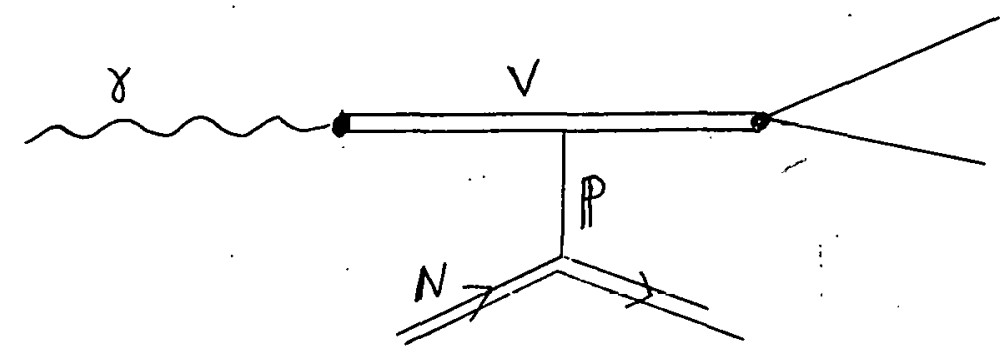

Figure 1.3

then the scattering amplitude should factor into the product of the amplitudes at each vertex in figure 1.3. This is because the Pomeron only carries the quantum numbers of the vacuum ( $I=S=B=0)$. This implies that excitation of the target nucleon should be independent of the mass and quark content of the scattered meson. The photoproduction of the $\phi$ and the $\psi$ vector mesons is particularly valuable for studying Pomeron exchange because $\phi \mathrm{P}$ and $\psi \mathrm{P}$ elastic scattering is exotic (no resonance possible) in the s-channel (assuming the $\phi$ and $\psi$ are pure $s \bar{s}$ and $c \bar{c}$ respectively) restricting t-channel exchanges to the Pomeron. 
'Section 4: High Energy Photoproduction of Vector Mesons

This thesis presents new results on the high energy photoproduction of three of the vector mesons, the $\rho^{\sigma}(770)$, the $\phi(1020)$, and the $\psi(3100)$ : Many of the results for $\psi$ photoproduction have been published already [12], so this thesis deals primarily with the high energy photoproduction of the $\rho$ and $\phi$. New results on diffractive inelastic $\psi$ photoproduction are then presented at the end in chapter 10. It should be emphasized that this is primarily a "measurement" thesis and not a "bump-hunt" thesis. The objective was to measure carefully, in a new kinematic regime, the properties of states known to exist from low energy experiments. This is the first time that the invariant mass distributions and 4 -momentum transfer squared $(t)$ distributions have been measured for photoproduced $\pi^{+} \pi^{-}$and $\mathrm{K}^{+} \mathrm{K}^{-}$final states above $20 \mathrm{GeV}$. We extend these measurements from $35 \mathrm{GeV}$ to $225 \mathrm{GeV}$. The opportunity to investigate a new kinematic regime should never be by-passed because very often new aspects of dynamics become visible. 


\section{Chapter II}

\section{The Experimental Apparatus}

Experiment 401 (E-401) was performed at Fermi National Accelerator Laboratory from February to December of 1979. The data were taken in the photon-enhanced broad band neutral beam in the Proton East experimental area. The three main goals of E-401 were:

1) Measurement of the $s$ and $t$ dependence of elastic $e, \omega, \phi$, and $\psi$ vector meson photoproduction

2) Measurement of the $\Psi^{\prime}(3.7)$-nucleon total cross section

3) Measurement of the photoproduced $\mathrm{K}^{+} \mathrm{K}^{-}, \mathrm{p}^{+} \mathrm{p}^{-}, \mu^{+} \mu^{-}$, and $e^{+} e^{-}$mass spectra off resonance for high sensitivity particle searches.

This thesis is primarily. concerned with the completion of objective number one.

The completion of these goals required producing: a high energy photon beam on target and building a multiparticle spectrometer for final state detection. Any of the decays ( $\rho \rightarrow \pi^{+} \pi^{-}, \omega \rightarrow \pi^{+} \pi^{+} \pi^{0}, \phi \rightarrow \mathrm{K}^{+} \mathrm{K}^{-}, \psi \rightarrow \mu^{+} \mu^{-}, \psi \rightarrow \mathrm{e}^{+} \mathrm{e}^{-}$, required charged track detection for their observation. The production angles and momenta of these charged particles were measured with a magnetic spectrometer consisting of 5 multiwire proportional chambers and 2 magnets. Distinguishing between, and among, hadrons,leptons and photons required added particle indentification capabilities. Particle identification was achieved using gas Cerenkov counters, steel-scintillator and lead-scintillator shower detectors, and a "fly's-eye" array of 
lead glass blocks. Elastically produced events were identified with a recoil proton detector suspended around a liquid hydrogen target. The rest of the apparatus was completed with various scintillation counters for triggering and vetoing purposes. Figure 2.1 shows a plan view drawing of the complete multiparticle spectrometer in proton East used for this experiment. The following sections describe the individual detectors in detail.

Section 1: The Neutral Beam

The $350 \mathrm{GeV}$ primary proton beam impinged on a $30.5 \mathrm{~cm}$ berylluim target 450 feet upstream of the experimental area. Charged particle secondaries were magnetically swept into a tungsten dump. The resulting neutral beam contained neutrons, $\mathrm{K}_{L}^{0}$ 's and photons from the decay of secondary $\pi^{\circ}$ 's. Two liquid deuterium filters, 11 meters and 23 meters long respectively, preferentially passed the photons and filtered other neutrals because of the high ratio of interaction length to radiation length for deuteruim. The beam emerged from the deuterium filters with a photon/neutron ratio enhanced by a factor of 200 . The resulting $1 " \times 2 "$ collimated photon beam had a residual 18 hadronic contamination. Figure 2.2 shows a drawing of the neutral beam line.

The residual 18 hadronic contamination was still a problem because the $20 \mathrm{mb}$ total cross section for hadroproduction is 200 times larger than the $110 \mu b$ total 

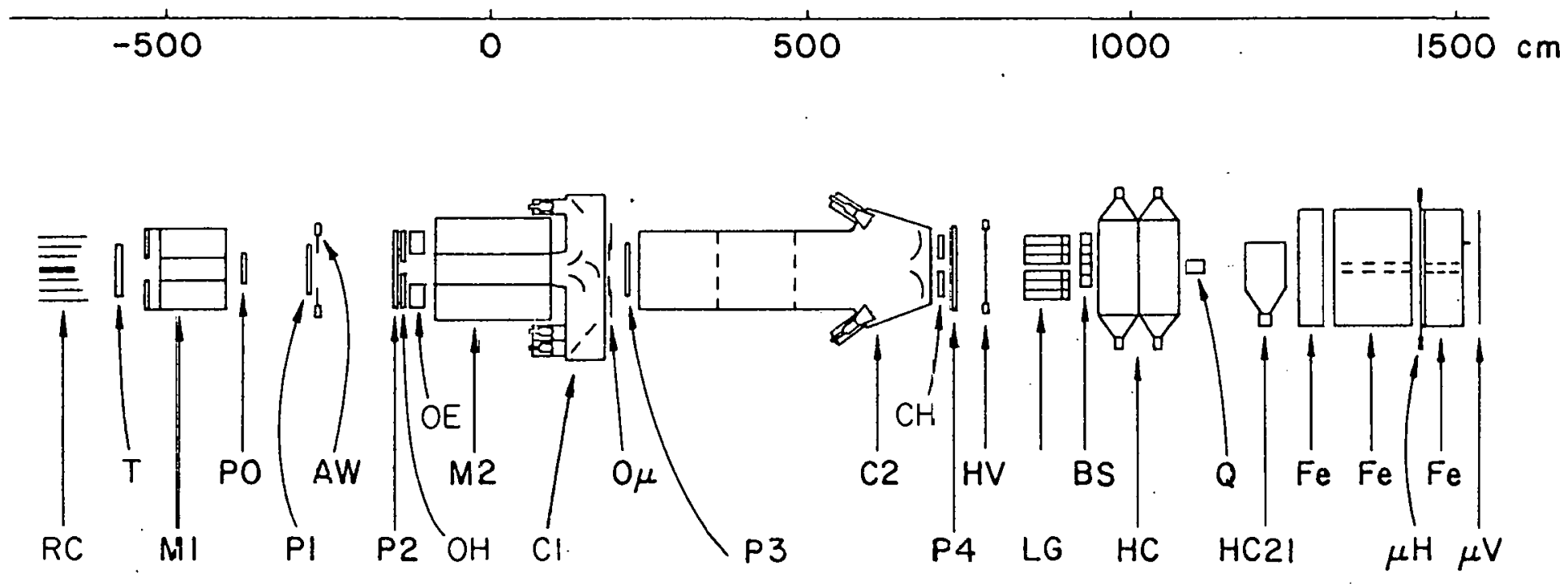

Figure 2.1 


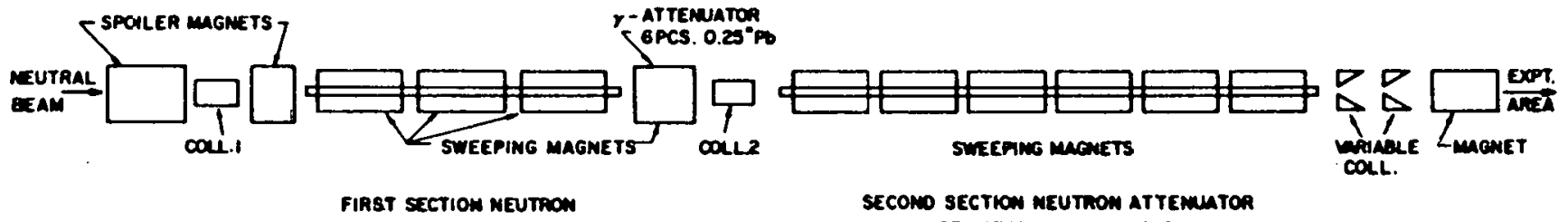
ATtEmutión 11 METEAS $\mathrm{D}_{2}$ CaYostat

SECONO SECTKON MEUTROW ATTEMUTTOR 
cross section for photoproduction[13]. The effects of the hadronic background were measured by inserting 6 radiation lengths of lead into the neutral beam. The lead attenuated the photon component of the beam by a factor 350 with respect to the attenuation of the hadronic component of the beam. The change in the trigger rate for our 2-track hadronic final state trigger, indicated that $25 \%$ of our events were hadron induced.

Figure 2.3 shows the photon spectrum measured by inserting a lead glass block into the beam. The lead glass block was calibrated with a low intensity momentum analyzed electron beam produced by inserting a thin lead foil into the photon beam. The photon beam power was recorded with a small wilson-type quantameter. A description of this quantameter can be found in reference [14].

Section 2: The Target

A liquid hydrogen $\left(\mathrm{LH}_{2}\right)$ target was used to facilitate the measurement of the $t$ distribution (4-momentum transfer) and $\mathrm{d} \sigma /\left.\mathrm{d} t\right|_{t=0}$ for low and high mass vector mesons. A target with a larger atomic number would cause many events to be produced coherently at small $t$, thereby depopulating the sample at larger $t$, and would obscure the measurement of $\mathrm{d} \sigma /\left.\mathrm{dt}\right|_{t=0}$. The use of a liquid hydrogen target had the advantage that the recoil nucleon was not trapped in the target material and could therefore be observed in a recoil detector. Further, 


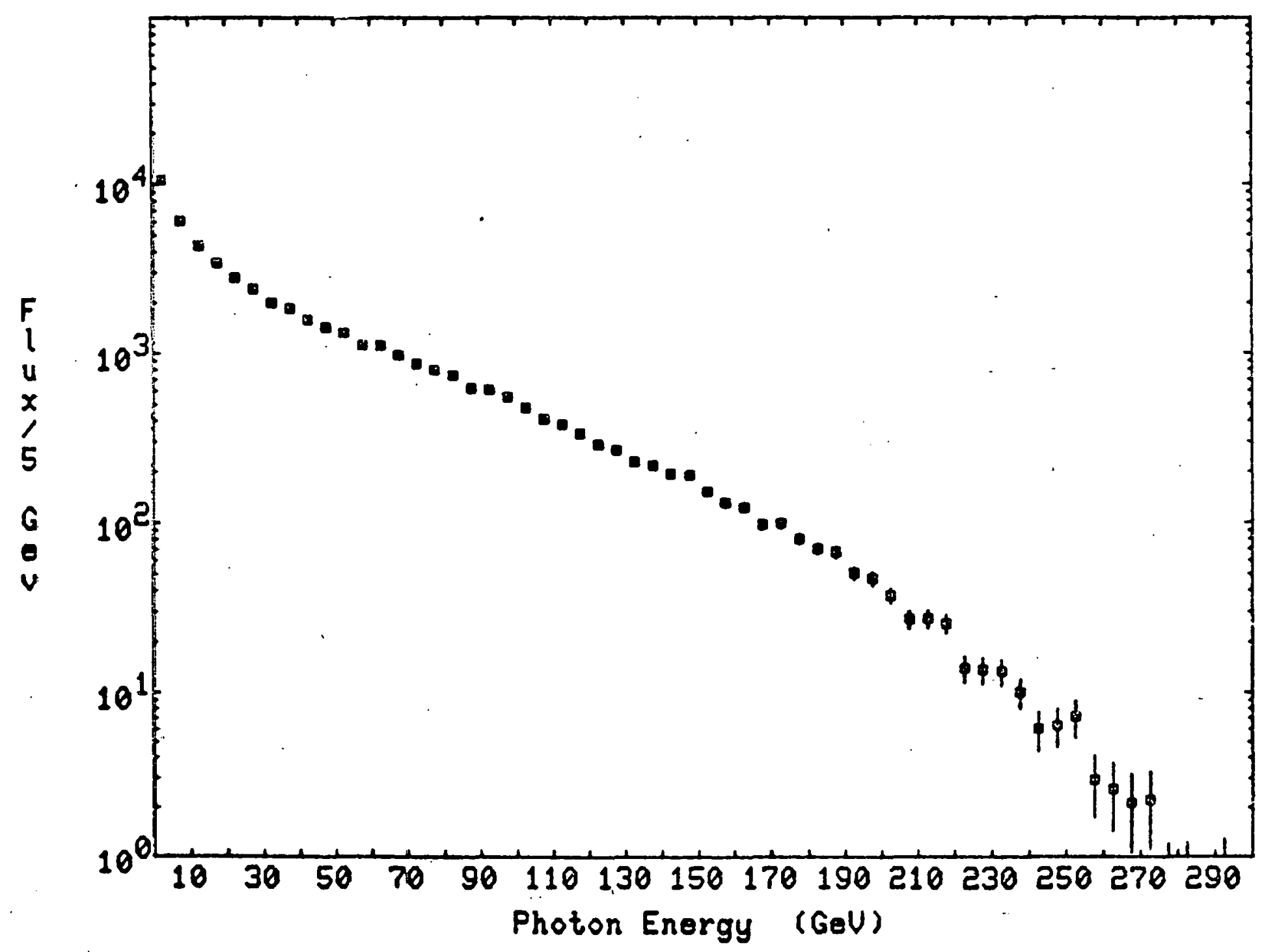

Figure 2.3 
the use of liquid hydrogen had the added benefit of having a small electromagnetic pair production cross section compared to higher $z$ materials. Electromagnetic production of $\mathrm{e}^{+} \mathrm{e}^{-}$ pairs is the major background to an experiment of this sort. The $\mathrm{e}^{+} \mathrm{e}^{-}$pair cross section is $\sim .58 \times \mathrm{z}^{2} \mathrm{mb}$ compared to the $110 \mu b$ cross section for the hadronic interactions of photons. Liquid hydrogen offered a very favorable interaction length/radiation length ratio to keep this background to a minimum. A diagram of our target is shown in figure 2.4. The 3.3 liter target presented $2.88 \mathrm{~g} / \mathrm{cm}^{2}$ of target material to the beam.

Section 3: The Recoil Detector

The recoil detector consisted of 24 scintillation counters and 12 lucite Cerenkov counters arrayed in three concentric hexagonal rings around the $\mathrm{LH}_{2}$ target. Figures $2.5 \mathrm{~A}$ and $2.5 \mathrm{~B}$ show the beam view and side view of this detector. The inner ring contained 6 19" $\times 22 " \times 1 / 4 "$ scintillation counters. The middle ring contained 18 $5 " \times 24 " \times 1 / 4 "$ scintillation counters. These 24 counters were observed with adiabatic plexiglass light pipes and 2" phototubes (RCA8575). The inner 2 rings provided azumithal angle resolution of $+/-.17$ radians for a recoil nucleon emerging from the $\mathrm{LH}_{2}$ target. The outer ring contained 12 11" $\times 27 " \times 1 "$ lucite Cerenkov counters with a threshold velocity $\beta=2 / 3$ providing $\pi / \rho$ identification. These were observed with 5" phototubes (RCA4522). The anode signals from 


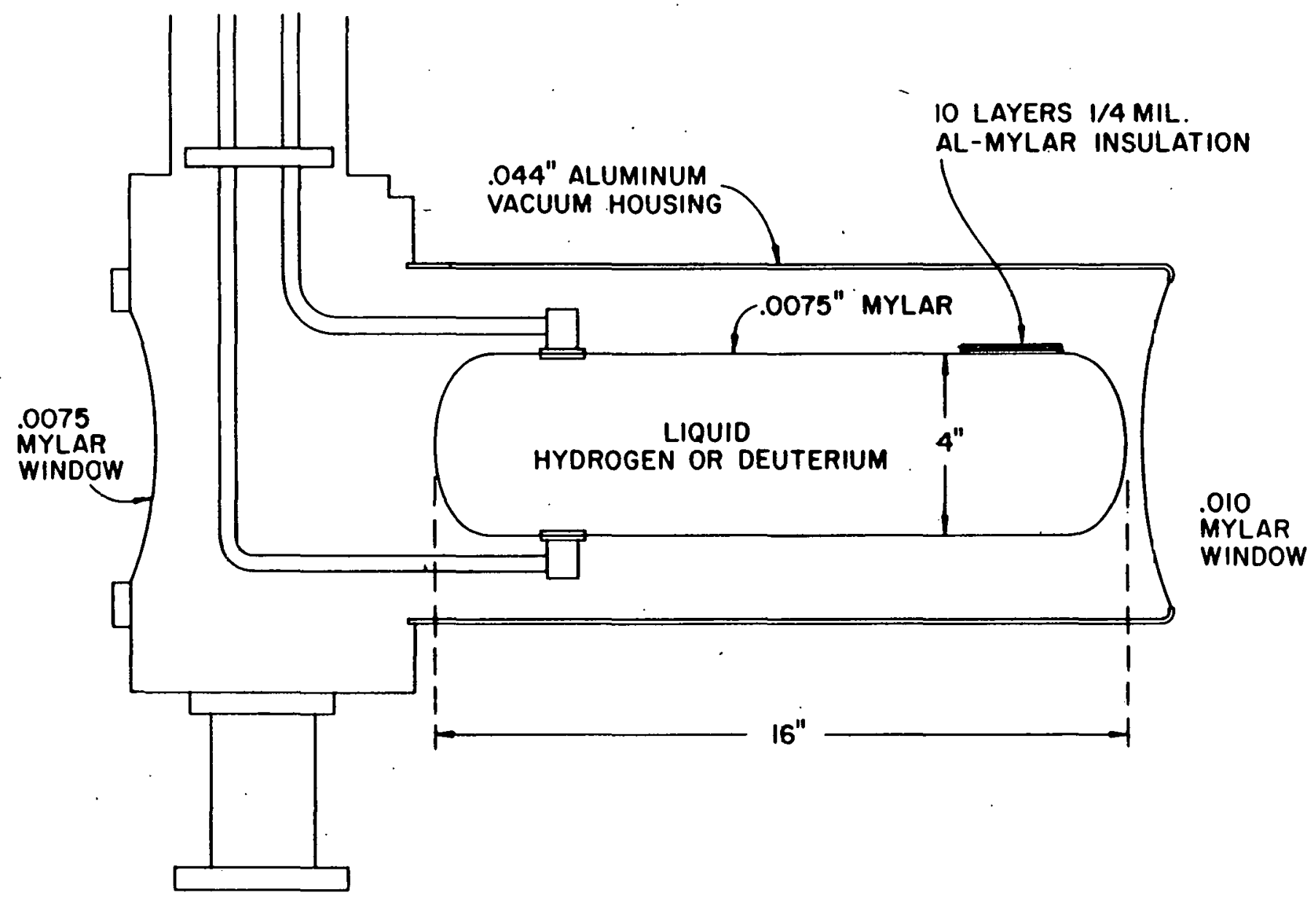




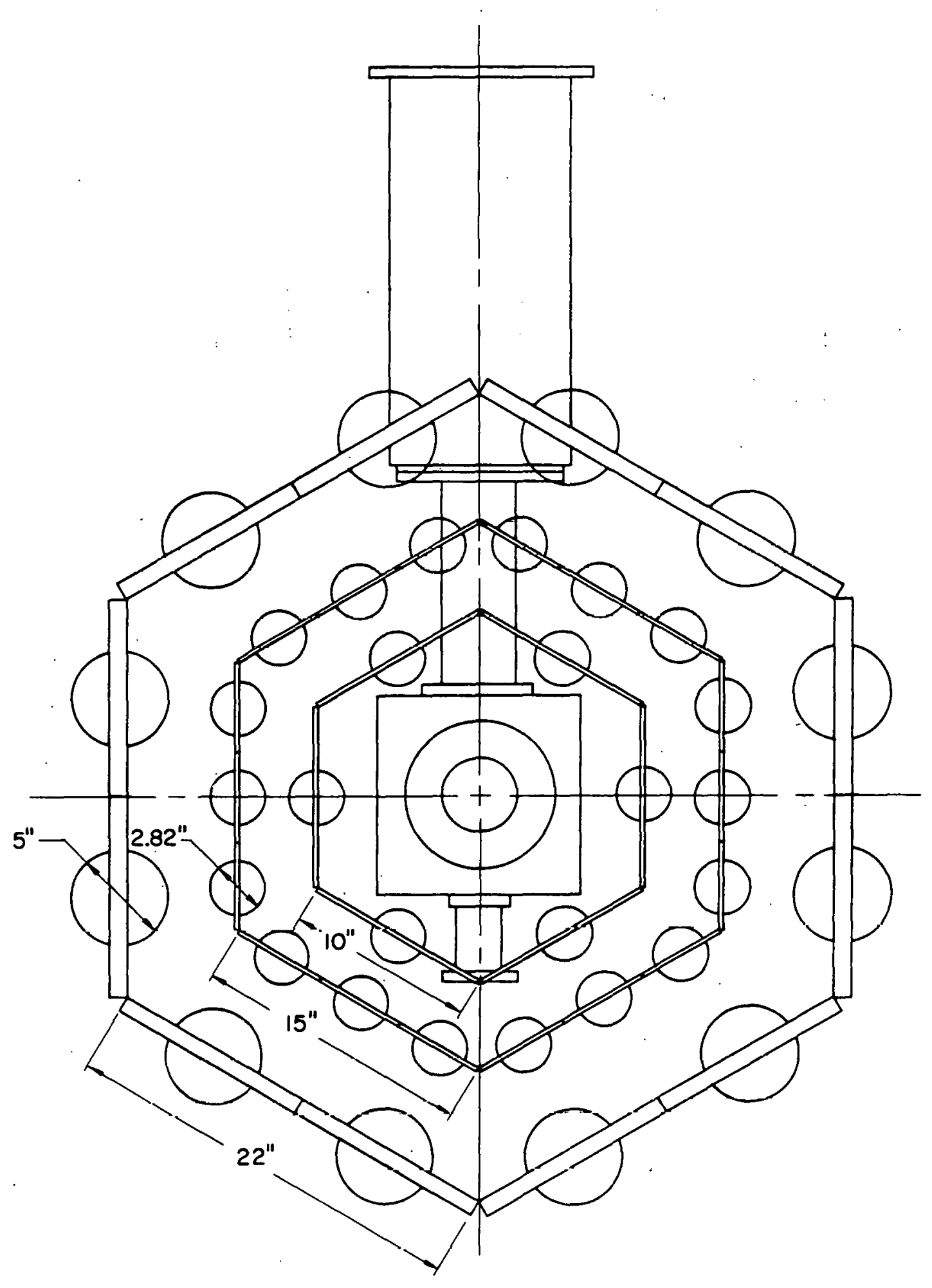

Figure $2.5 \mathrm{~A}$ 


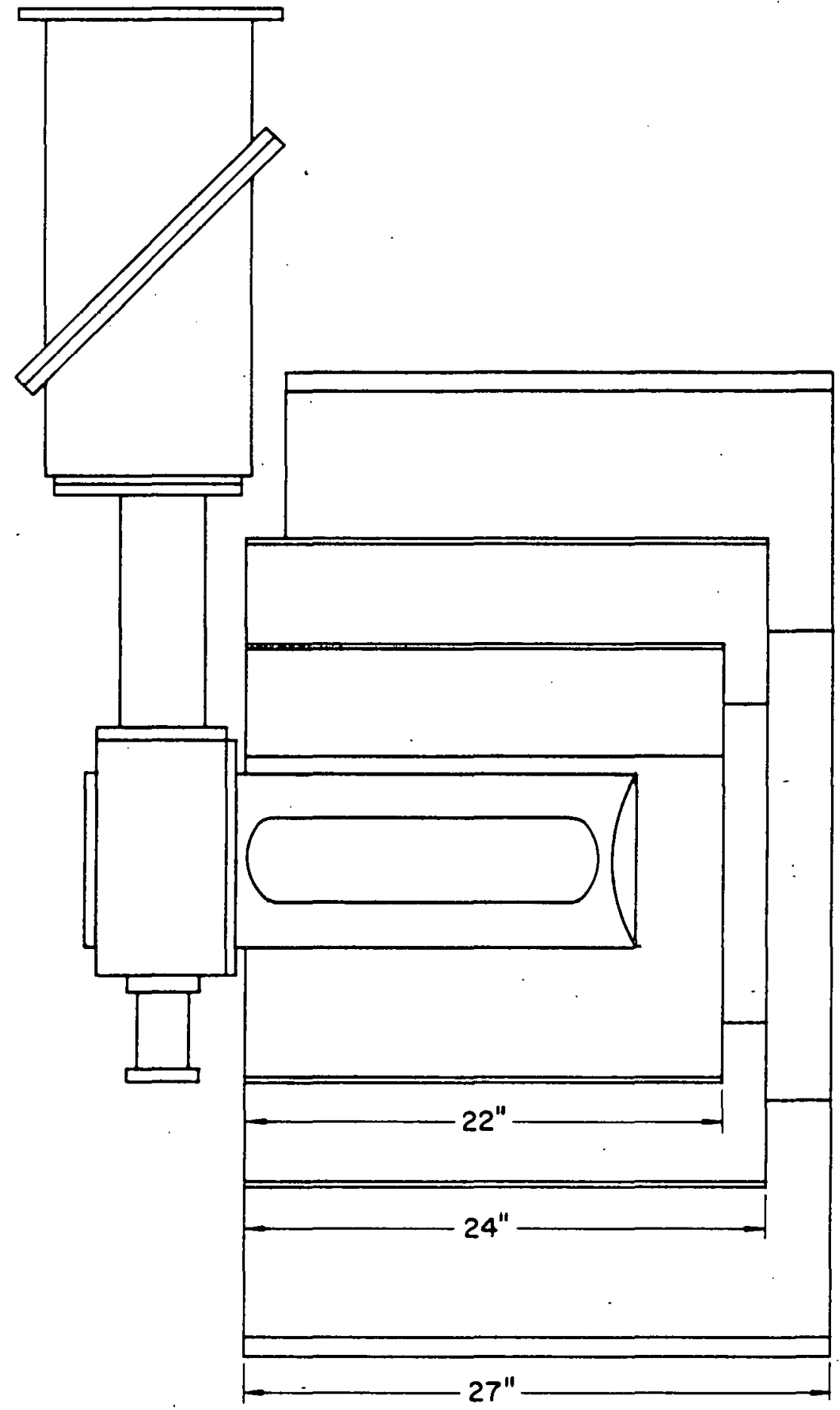

Figure 2.5B 
the scintillation counters in the two inner rings were discriminated at $30 \mathrm{MV}$ and latched at the coincidence register. The dynode signals from these counters were ganged together in groups of 3 and recorded by ADC's. The anode signals from the outer ring were recorded by ADC's. The analysis of this detector is described in detail in chapter VI.

Section 4: Charged Track Detection

The magnetic spectrometer consisted of 5 multiwire proportional chambers ( $\mathrm{P} \emptyset-\mathrm{P} 4$ in figure 2.3$)$ and two vertical bending magnets ( $M I$ and M2 in figure 2.3) downstream of the target. Each chamber contained 3 planes of wires $X, V$ and $U$. The vertical $x$ wires measured coordinates in the non-bend plane. The $\mathrm{V}$ and $\mathrm{U}$ wires were inclined to the horizontal by $-1+11.31^{\circ}$ respectively for a stereoscopic measurement in the bend plane. Table 2.1 summarizes the MWPC properties. A detailed description of their construction can be found in $[15]$.

The chambers were used for triggering purposes by grouping together "bands" of wires, 32 bands/chamber, and using the logical "OR" from each band at a fast logic rack to make a crude multiplicity bussline.

Tracks making it through both bending magnets and all 5 chambers are called "inner" tracks. Tracks going through MI and $\mathrm{P} \emptyset-\mathrm{P} 2$ (see figure 2.3) only are called "outer" tracks. 
Tab1.è 2.1

\begin{tabular}{|c|c|c|c|c|c|}
\hline \multirow{2}{*}{$\begin{array}{l}\text { Chamber } \\
\text { Po }\end{array}$} & \multirow{2}{*}{$\begin{array}{l}\text { Aperture } \\
11.7^{\prime \prime} \times 16.6^{n}\end{array}$} & \multirow{2}{*}{$\begin{array}{l}\text { Distance } \\
\text { from M2 } \\
-155.7^{\prime \prime}\end{array}$} & \multicolumn{2}{|c|}{$\begin{array}{r}\text { Number } \\
\text { wires }\end{array}$} & \multirow{2}{*}{$\begin{array}{l}\text { Wire } \\
\text { spacing } \\
.04^{n} \\
.04^{n} \\
.04^{n}\end{array}$} \\
\hline & & & $\begin{array}{l}288 \\
416 \\
416\end{array}$ & $\begin{array}{l}X \\
V \\
U\end{array}$ & \\
\hline P1 & $20.4^{\prime \prime} \times 30.0^{n}$ & -112.11 & $\begin{array}{l}256 \\
384 \\
384\end{array}$ & $\begin{array}{l}X \\
V \\
U\end{array}$ & $\begin{array}{l}.08^{n} \\
.08^{n} \\
.08^{n}\end{array}$ \\
\hline P2 & $30.8^{\prime \prime} \times 45.2^{n}$ & $-61.4^{\prime \prime}$ & $\begin{array}{l}384 \\
576 \\
576\end{array}$ & $\begin{array}{l}X \\
V \\
U\end{array}$ & $\begin{array}{l}.08015^{n} \\
.08015^{n} \\
.08015^{n}\end{array}$ \\
\hline P3 & $32.8 n \times 44.4^{n}$ & $82.6^{n}$ & $\begin{array}{l}416 \\
576 \\
576\end{array}$ & $\begin{array}{l}X \\
V \\
U\end{array}$ & $\begin{array}{l}.07874^{n} \\
.07863^{n} \\
.07863^{n}\end{array}$ \\
\hline P4 & $39.8^{\prime \prime} \times 60.1^{n}$ & $292.1^{n}$ & $\begin{array}{l}336 \\
768 \\
768\end{array}$ & $\begin{array}{l}X \\
V \\
U\end{array}$ & $\begin{array}{l}.11895^{n} \\
.08 n \\
.08 n\end{array}$ \\
\hline
\end{tabular}


For this reason these two regions of the detector are referred to as the "inner detector" and the "outer detector". This thesis is concerned with events in the inner detector only.

Section 5: Trigger Counters

5a) Inner Detector

Figure 2.6 shows the highly segmented T-counter (target counter) located just downstream of the $\mathrm{LH}_{2}$ target and upstream of Ml. The T-counter provided a fast trigger signal flagging charged particles emerging from the target. off-line, the segmentation of the T-counter provided a measurement of the bend plane coordinate upstream of $\mathrm{Ml}$ improving the vertex resoltution for tracks in the outer detector.

Figure 2.7 shows the Cerenkov counter hodoscope (CH). The $\mathrm{CH}$ counters were mounted directly behind, and with a 1-to-l correspondence to, the mirrors of Cerenkov counter $C_{2}$. A heavy charged particle candidate was flagged when the $C_{2}$ mirror was "off" and the associated $\mathrm{CH}$ counter was "on". A detailed description of the "heavy particle" bussline can be found in reference [16].

The HxV trigger hodoscope is shown in figure 2.8. Located just downstream of $\mathrm{P} 4$, the HV counters were mounted 12 horizontally and 8 vertically with a $1 "$ gap in the middle to prevent triggering the apparatus on $e^{+} e^{-}$pairs. Section $7 a$ descrihes how the HV counters were used in coincidence with 


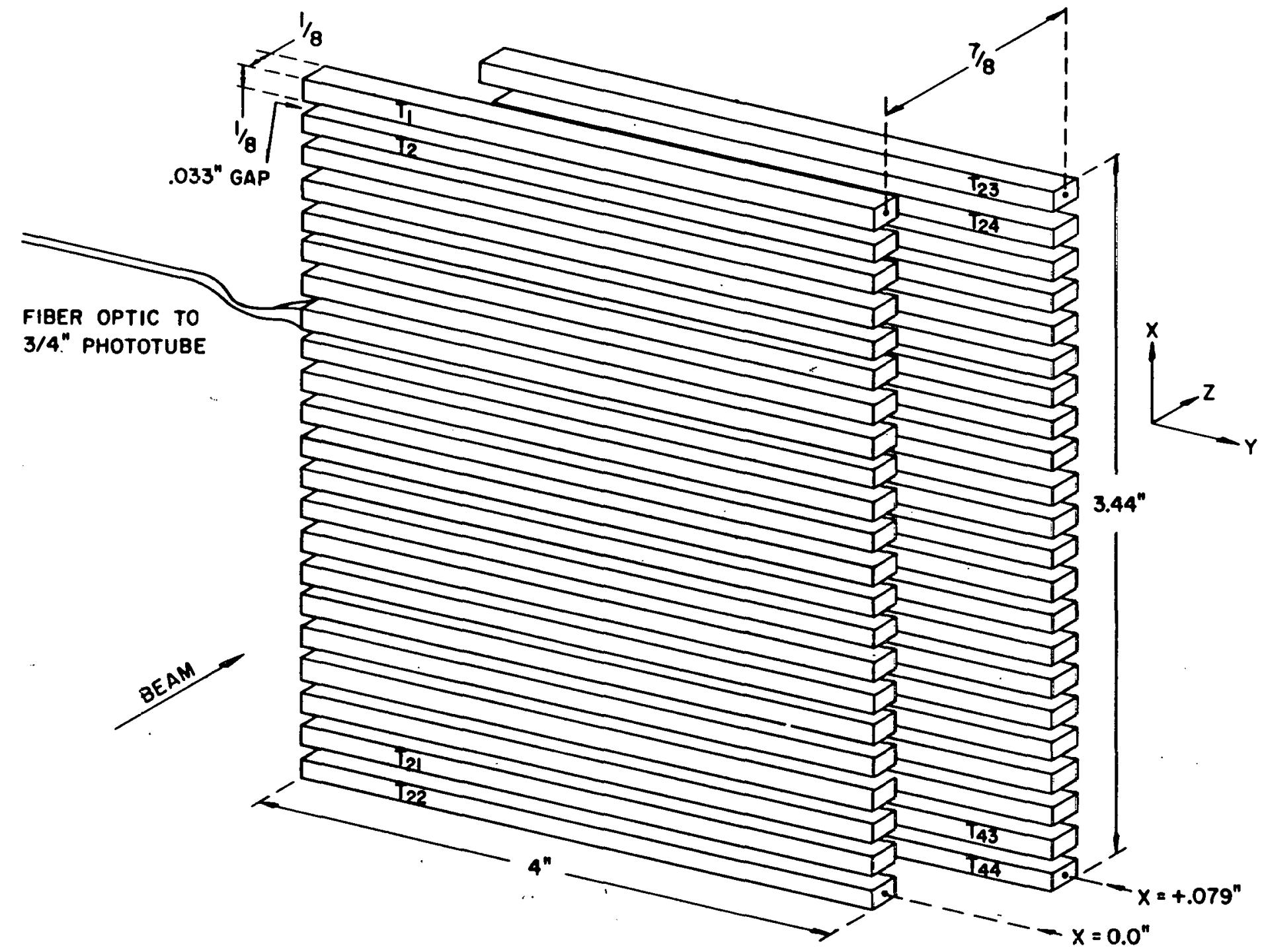

Figure 2.6 


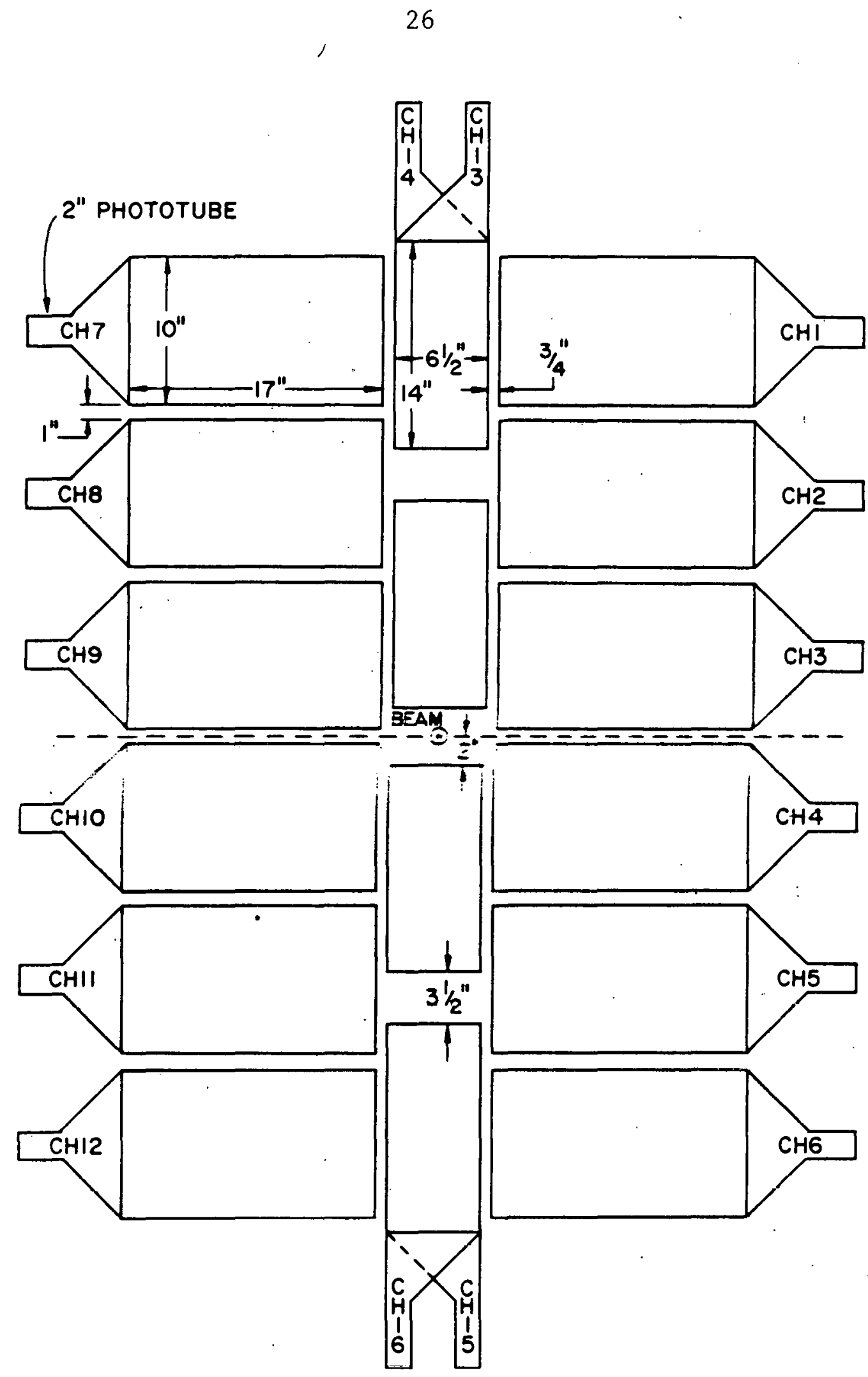

Figure 2.7 


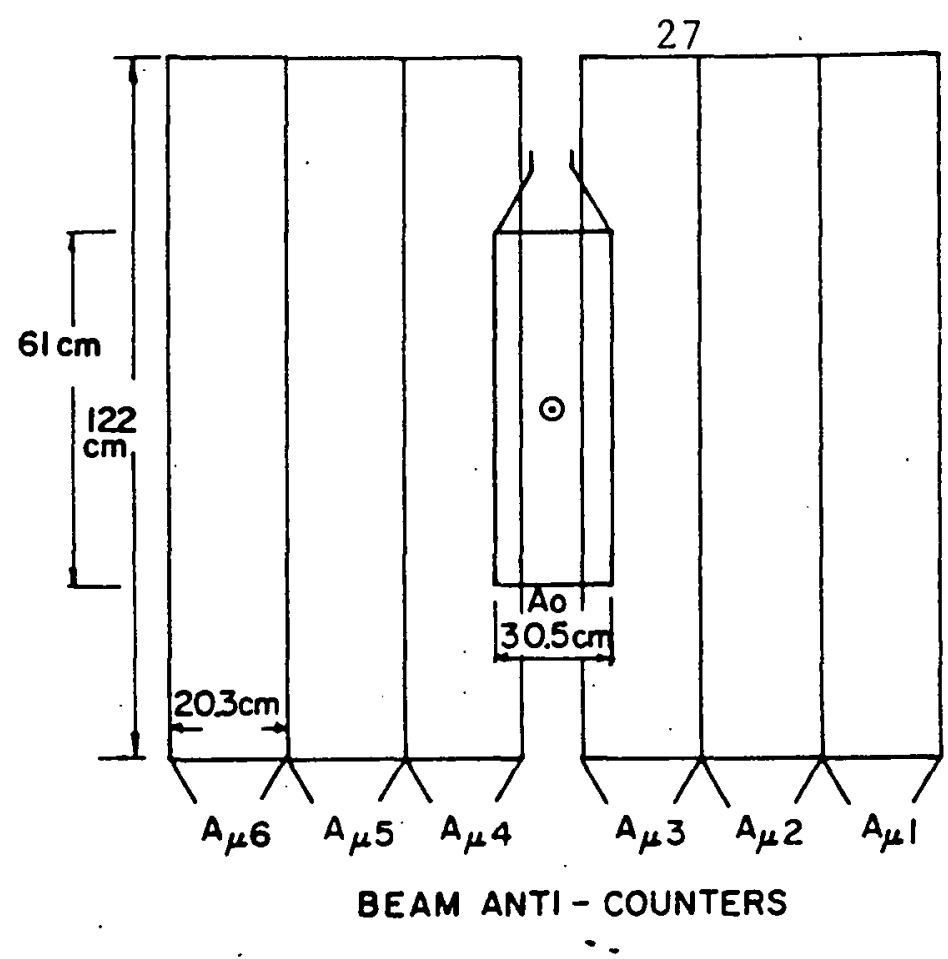

(a)

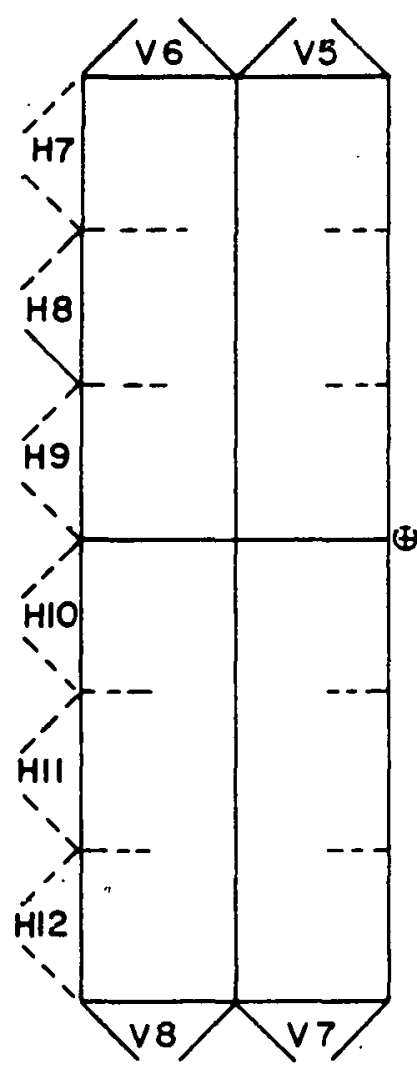

(va)

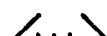


the T-counters to form the 2-body master gate for the inner detector.

\section{5b) Outer Detector}

Figure 2.9 shows the outer detector trigger counters $(\mathrm{OH})$. Mounted just, downstream of P2, the $\mathrm{OH}$ counters were used in coincidence with the T-counters to generate a 1 body master gate for the outer detector.

\section{5c) Veto Counters}

Figure 2.10 shows the AW (anti-wide angle) counters which were mounted around the perimeter of Pl. Charged tracks striking. the AW counters miss $\mathrm{Pl}$ and are not reconstructed as outer detector tracks. A bussline was formed as the logical "OR" of the 4 AW counters which was subsequently used as a veto for a 2-track inner detector event in this analysis. Figure 2.8 shows the 7 beam anti-counters that were located just upstream of the $\mathrm{LH}_{2}$ target. A coincidence between any of these counters and a downstream event flagged an event that was initiated by a charged particle from the beam halo (muons) and not by a photon, or by a muon in accidental coincidence with a photon-induced event. A correction for accidental vetos is made (see appendix $\mathrm{C}$ ). 


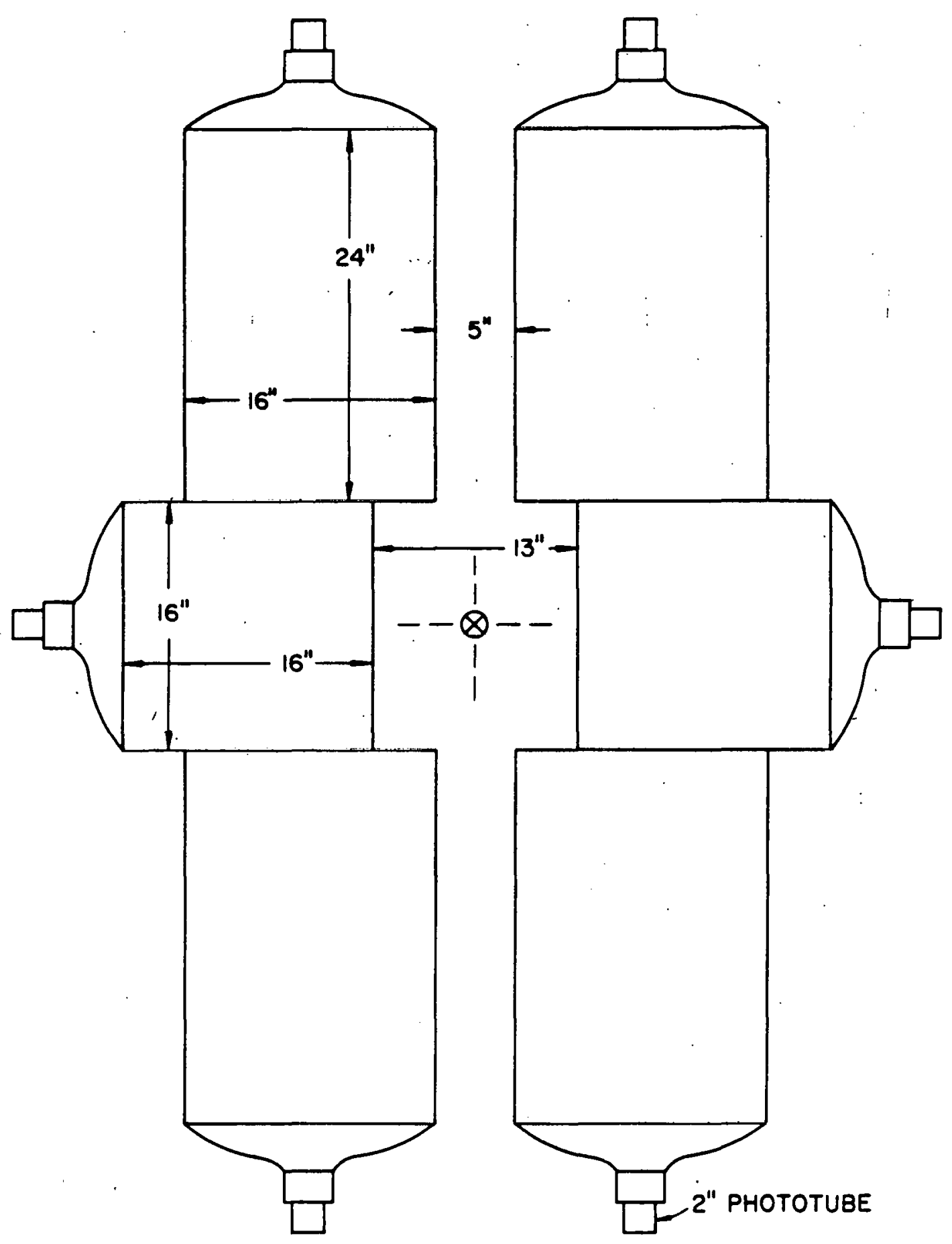

Figure 2.9 


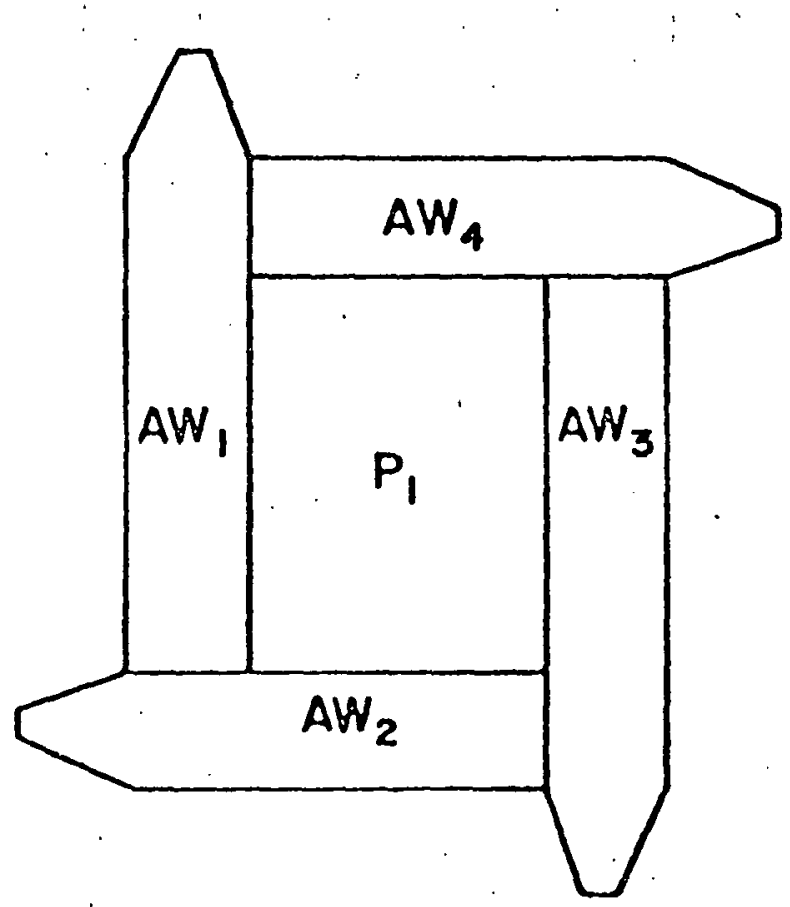

Figure 2.10 
Section 6: Particle Identification System

\section{6a) Inner Detector}

Two Cerenkov counters, $C_{1}$ and $C_{2}$, provided charged particle identification for the inner detector. $C$, (see figures 2.11A to 2.11B) is a 12-celled nitrogen filled counter with a $\pi / \mathrm{K} / \mathrm{P}$ threshold of $5.94 / 21.0 / 39.9 \mathrm{GeV} / \mathrm{c}_{0} \cdot \mathrm{C}_{2}$ (see figures $2.12 \mathrm{~A}$ to $2.12 \mathrm{~B})$ is a 16 -celled 208 nitrogen $80 \%$ heluim filled counter with a $\pi / K / P$ threshold of $10.7 / 37.8 / 71.9 . \mathrm{GeV} / \mathrm{C}$. Figure 2.13 shows the observed $\pi / \mathrm{K} / \mathrm{P}$ thresholds for each counter. The reader is referred to reference [16] for details of the Cerenkov system and the Cerenkov analysis.

The lead glass array (see figure 2.14), located behind P4, provided neutral particle detection. The array consisted of $422.5 " \times 2.5 " \times 23 "$ blocks surrounded by 48 6" $\times 6 " \times 18 "$ blocks. A 3.5" gap in the center of the array prevented "darkening" the lead glass by $\mathrm{e}^{+} \mathrm{e}^{-}$pairs and non-interacting photons. The reader is referred to reference [17] for a detailed description of the lead glass and its properties.

The back shower counters (BS) were located directly behind the lead glass gap. Stacked 8 high, these 6" $x 20 " 16$ radiation length lead-scintillator sandwich shower counters were used to attenuate the $e^{+} e^{-}$flux into the hadron calorimeter. Reference [18] contains more information on the BS counters. 

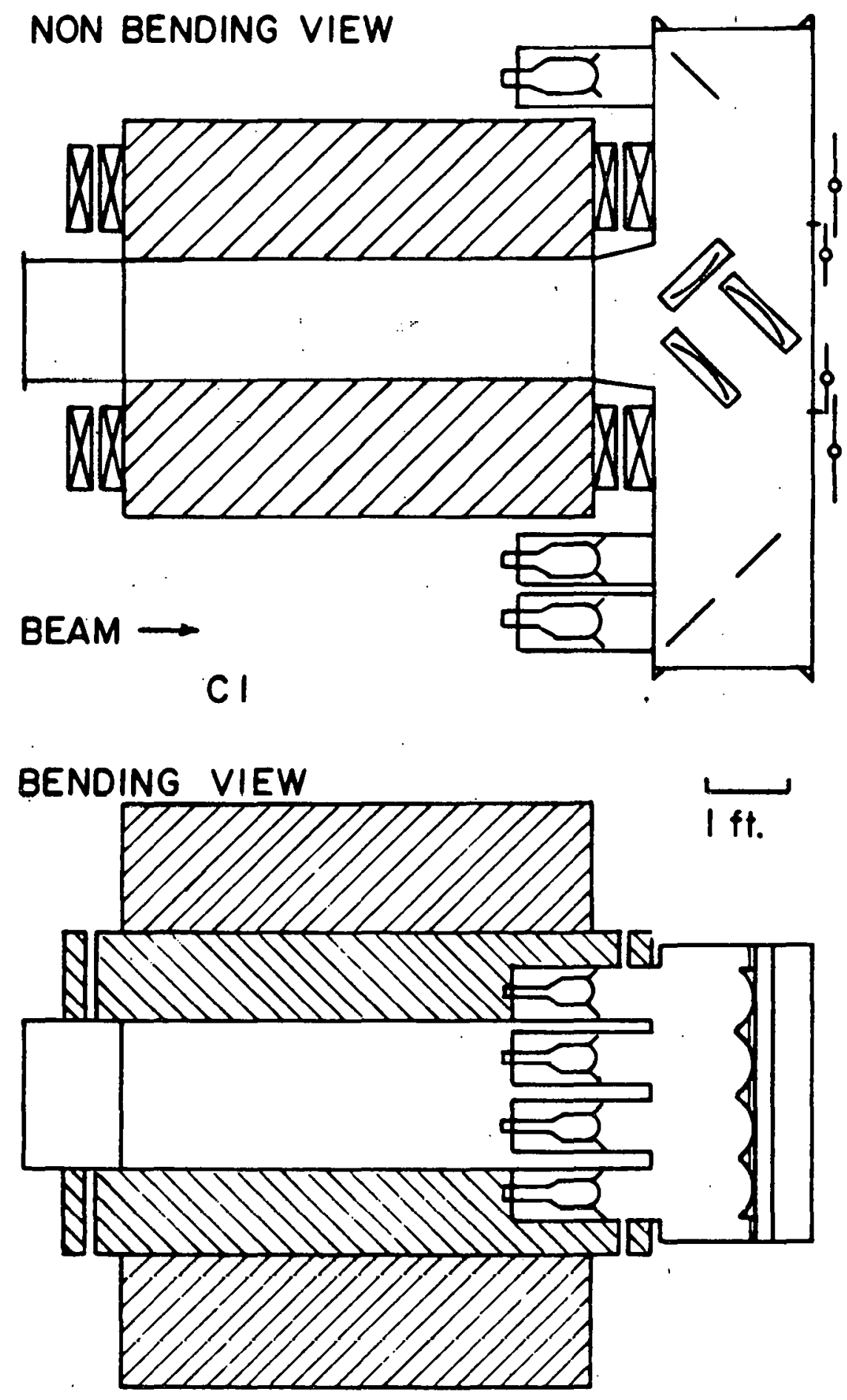

Figure 2.11A 
33

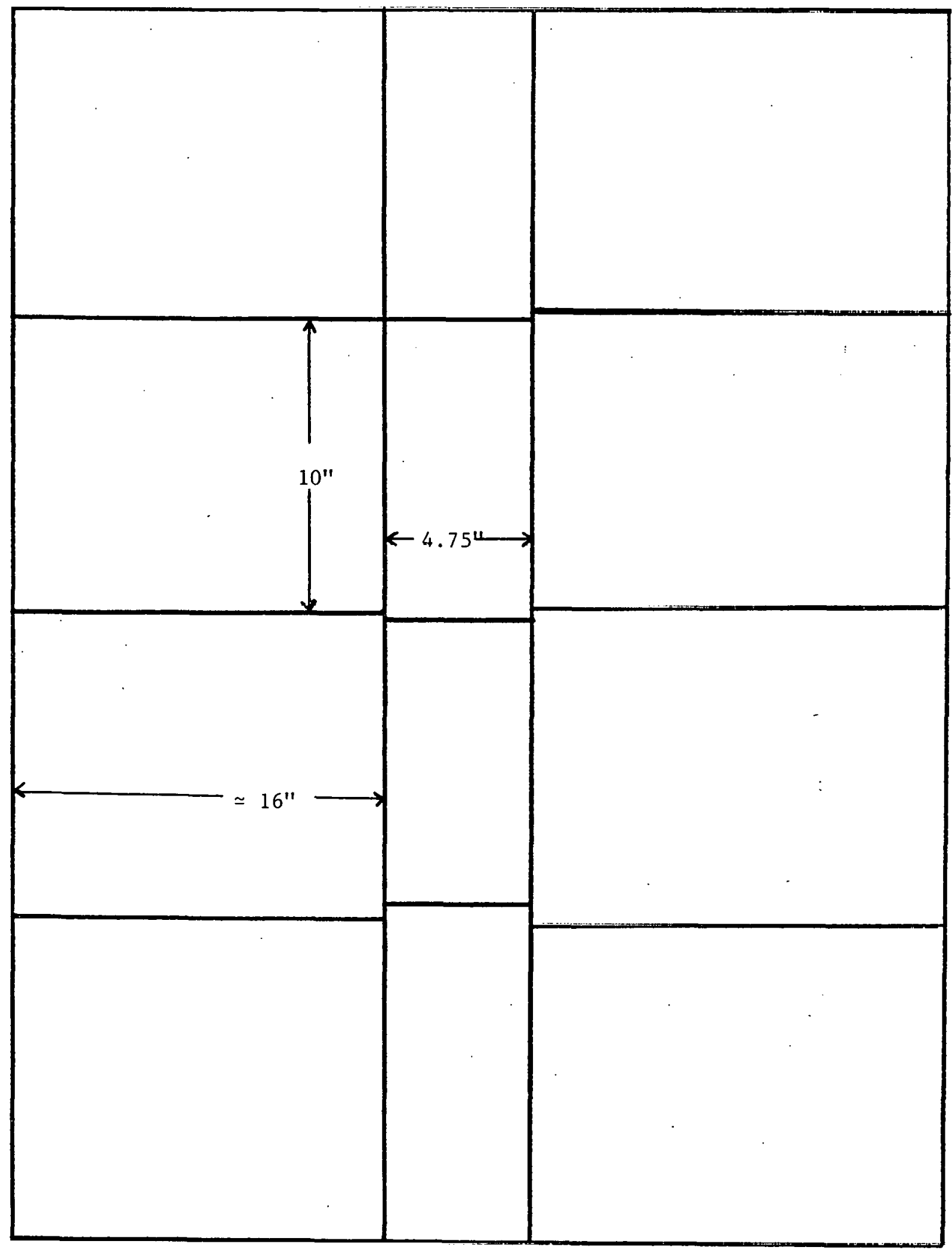

Figure 2.11B 


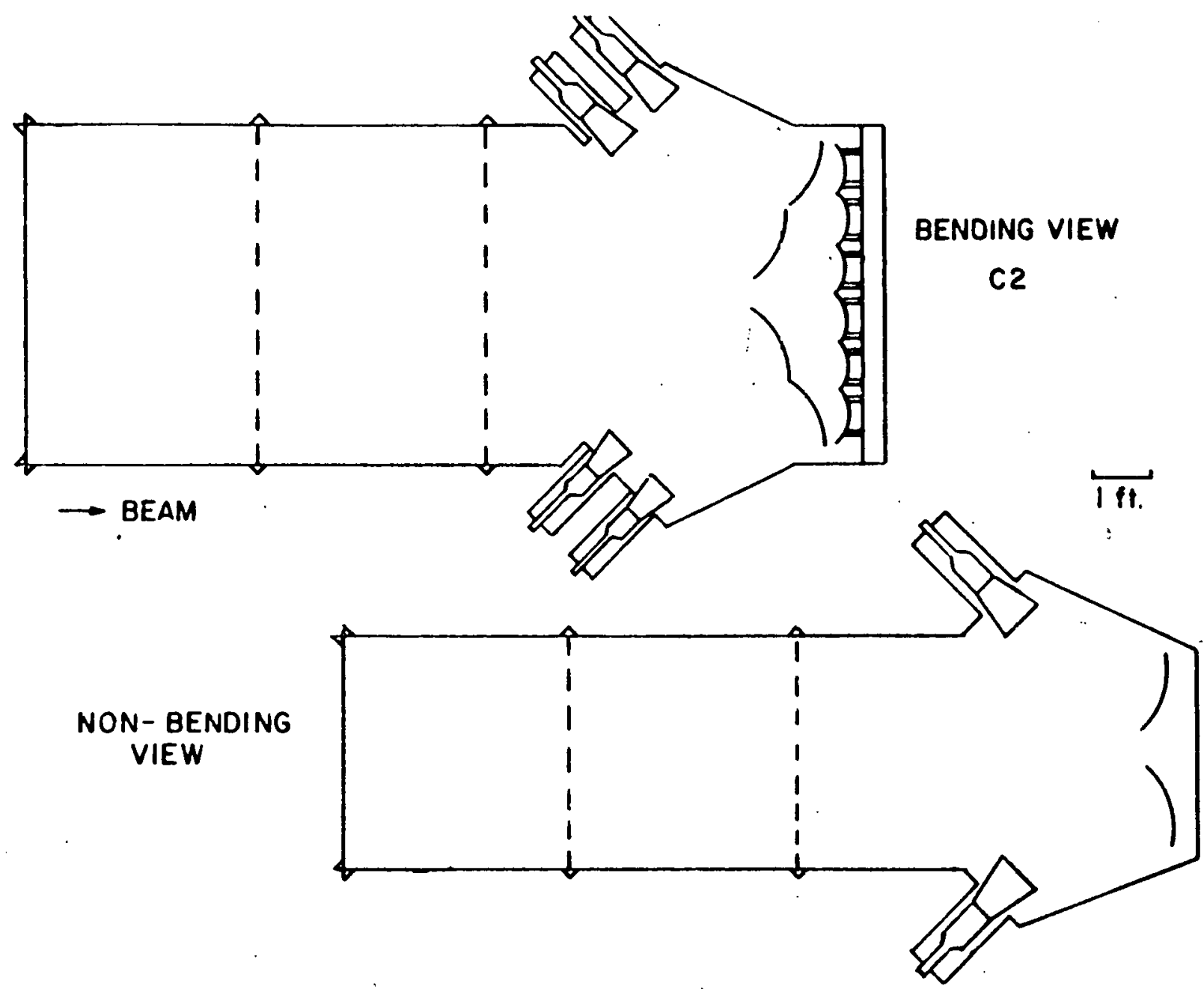




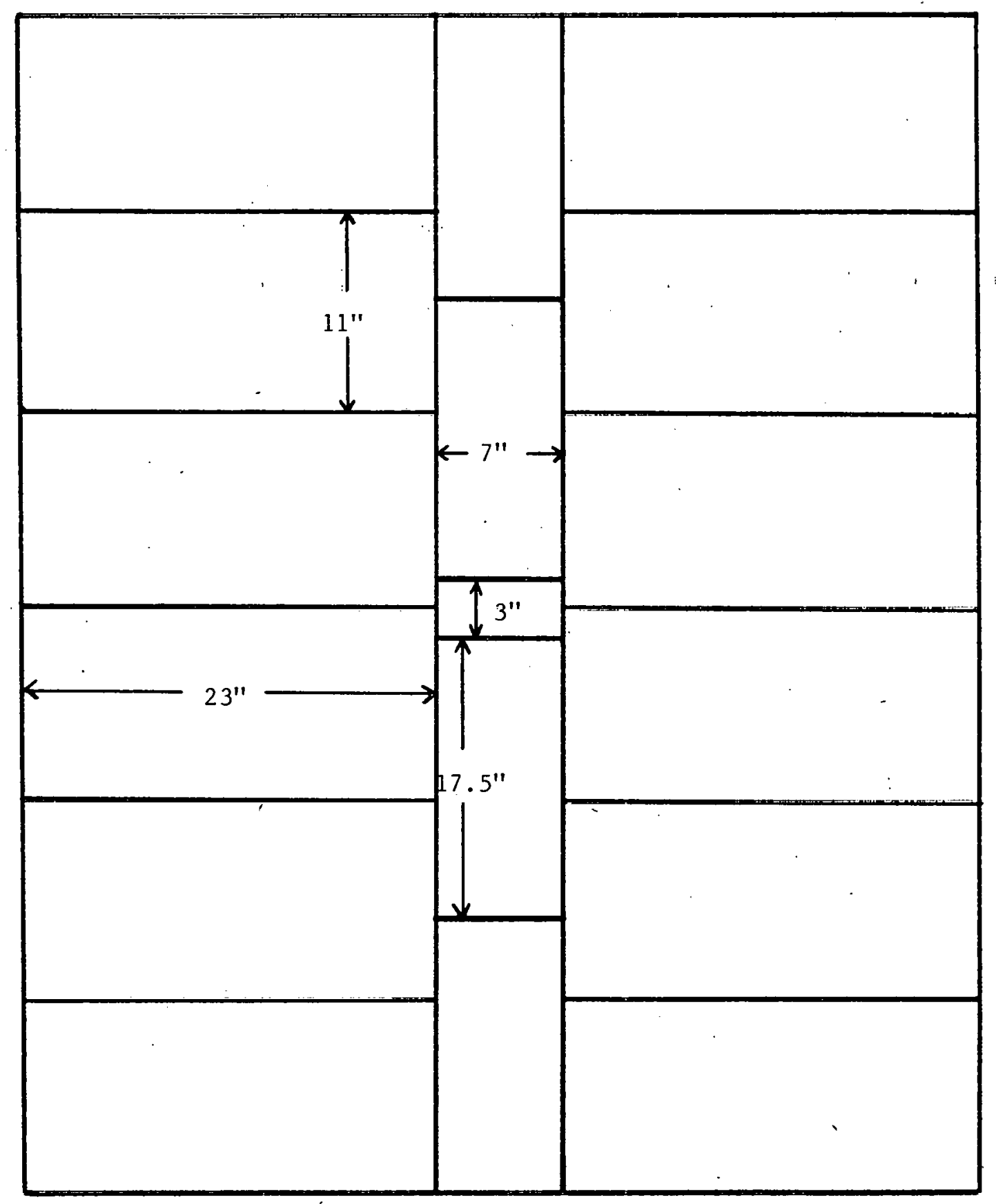

Figure 2.12B' 


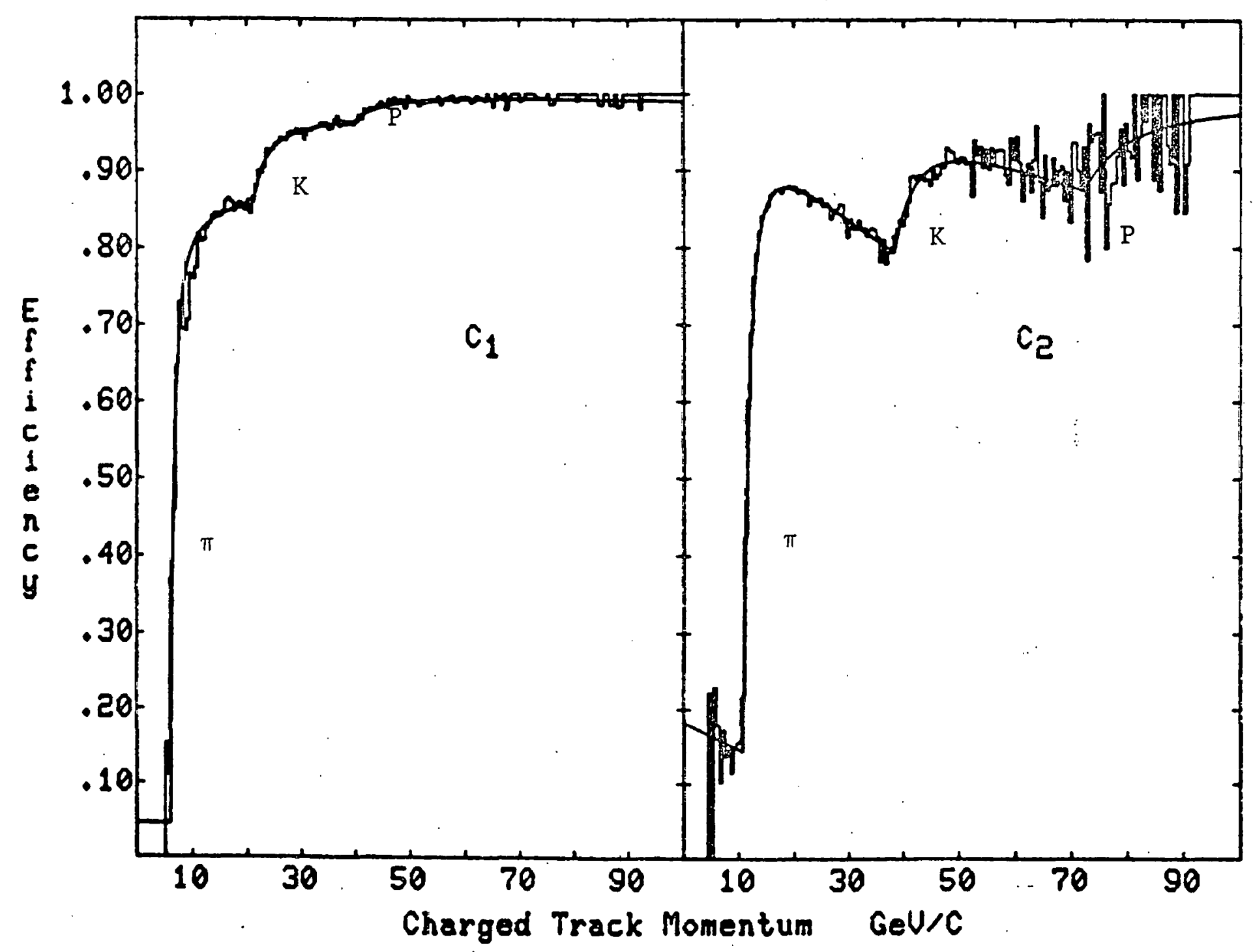

Figure 2.13 


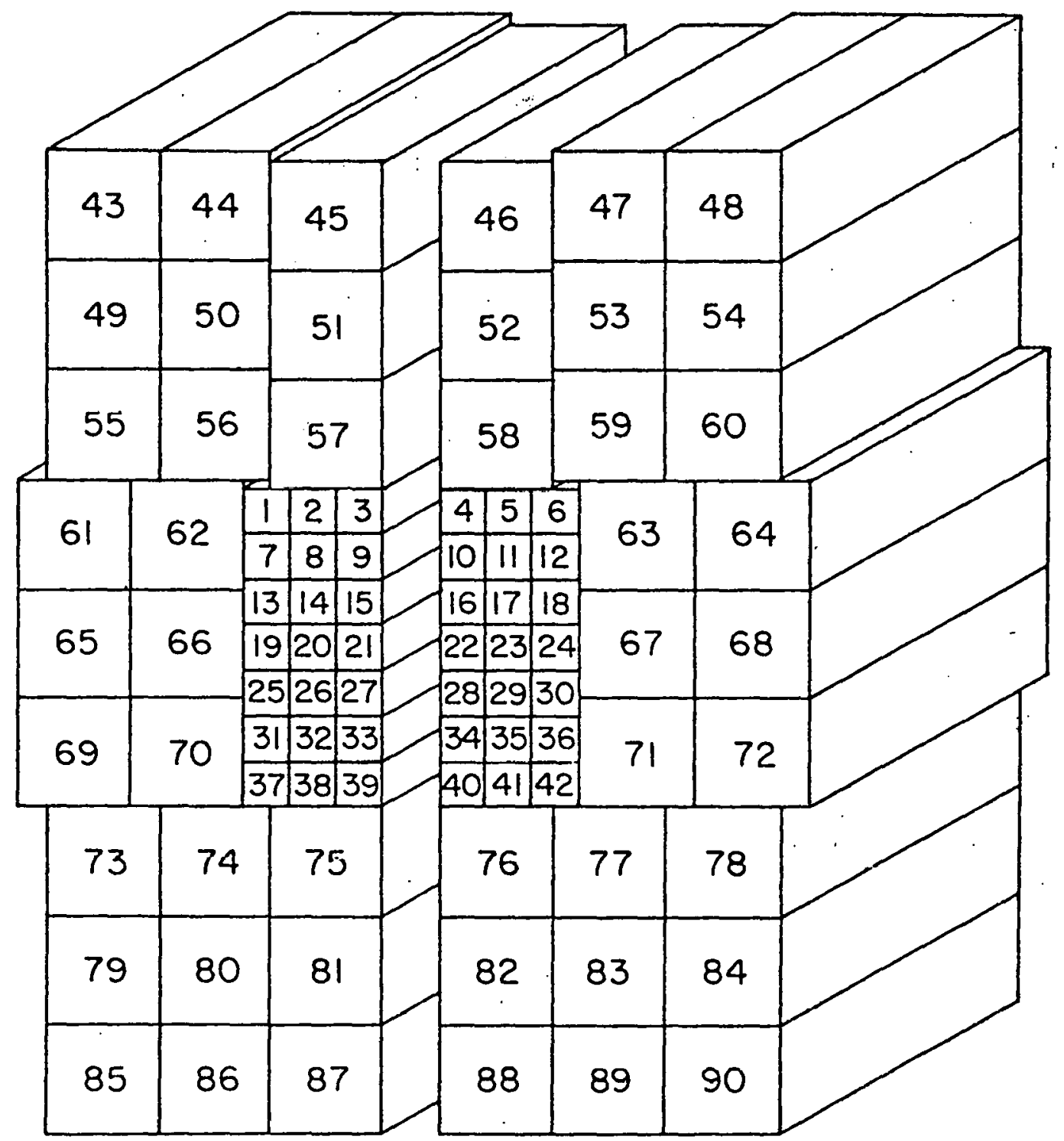

Figure 2.14 
The hadron calorimeter (HC) shown in figure 2.15 consisted of 20 steel-scintillator sandwich modules stacked five on a side and two deep. A 6" $\times 6 "$ hole in the middle allowed the passage of the primary photon beam to the quantameter directly behind it. Each module consisted of 12 1.75" steel plates interleaved with .25" scintillator sheets fanned into an optical mixing block viewed with a 5 " phototube (Amperex 58AVP). Another module was placed directly behind the quantameter to capture any hadronic energy lost down the hole. The HC was used to form the hadronic energy bussline for the trigger (see section 7c).

Muon identification is provided at the end of the inner detector by the $\mu \mathrm{H}$ (horizontal) and $\mu \mathrm{V}$ (vertical) scintillation counter arrays (figure 2.16). The $\mu \mathrm{H}$ - and $\mu \mathrm{V}$ counters are sandwiched between and behind large steel blocks that absorb everything else.

6h) Outer Detect.or

Photon and electron identification was provided by the outer electron detector (OE) located just upstream of M2 (see figure 2.17). Arrayed on each side of the M2 aperture were 6 6" $\times 20 " 3.4$ radiation length lead scintillator sandwich shower counters stacked horizontally in front of one 18 " $x$ " 35 " 18.4 radiation length lead-scintillator sandwich shower counter deployed vertically. Shower development in the first 3.4 radiation length section provided $e / \pi$ rejection. Stray 


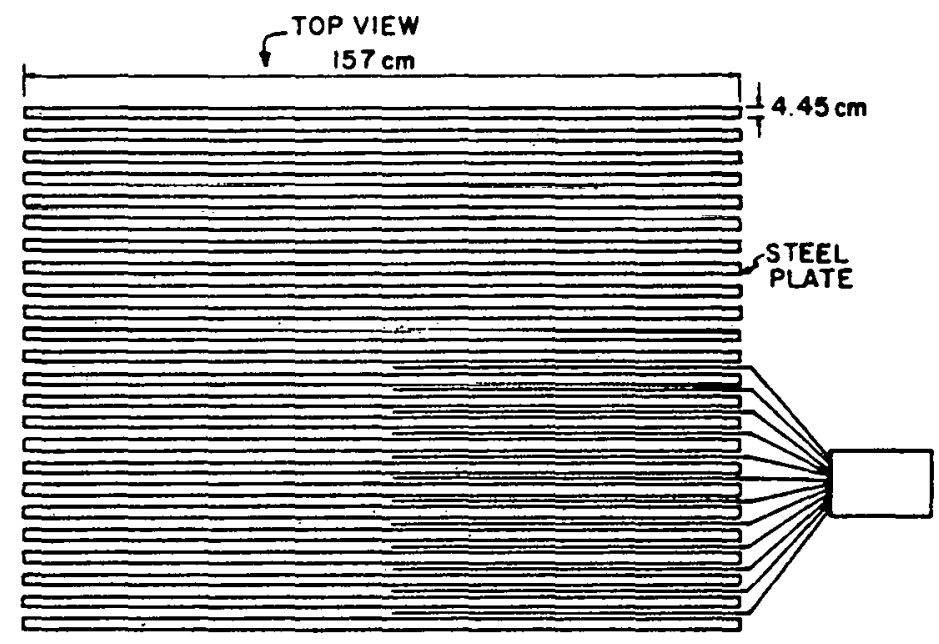

BEAM VIEW OF HADRON CALORIMETER

W WITH DETECTOR ARRANGEMENT SHOWN

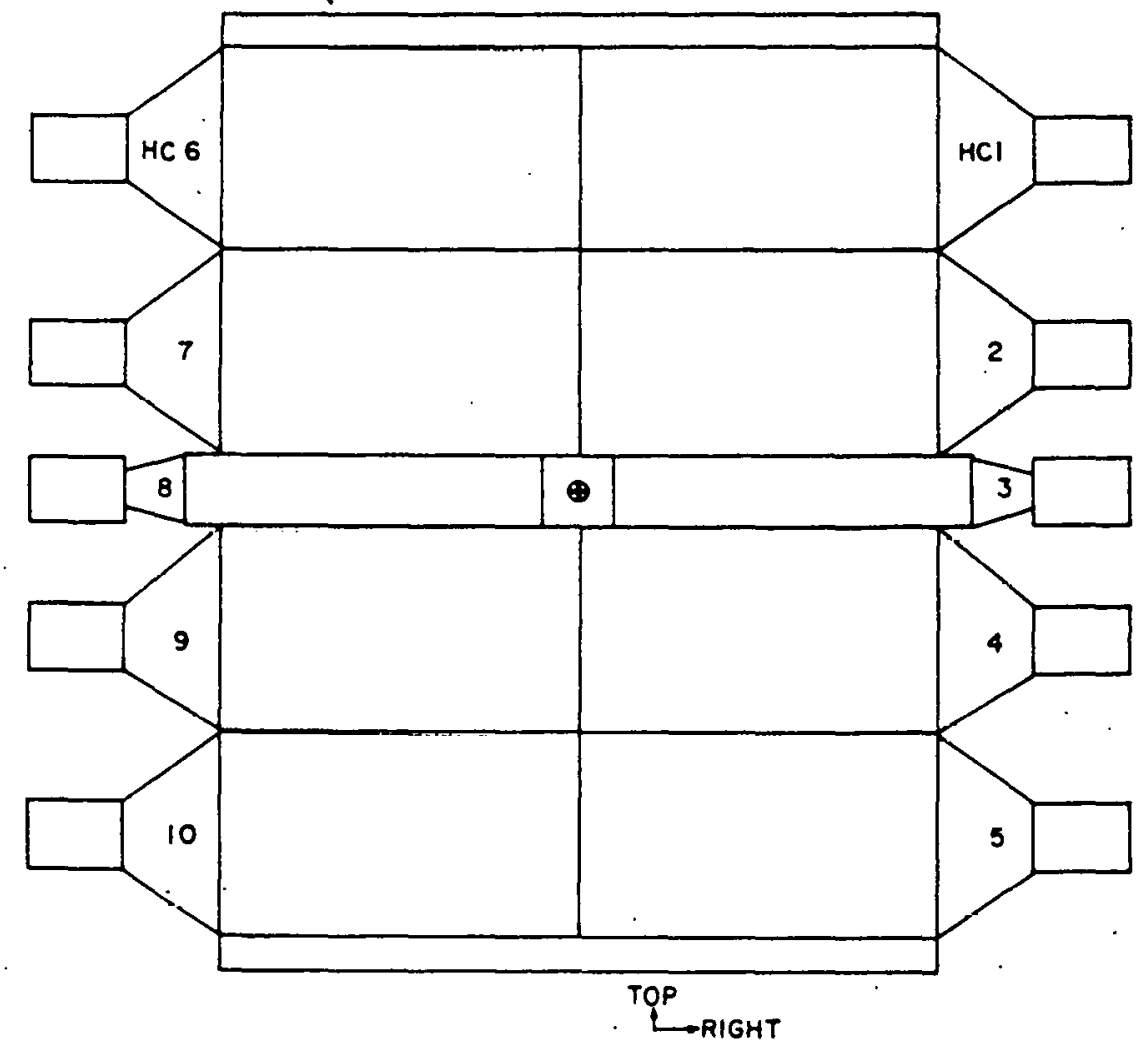

Figure 2.15 


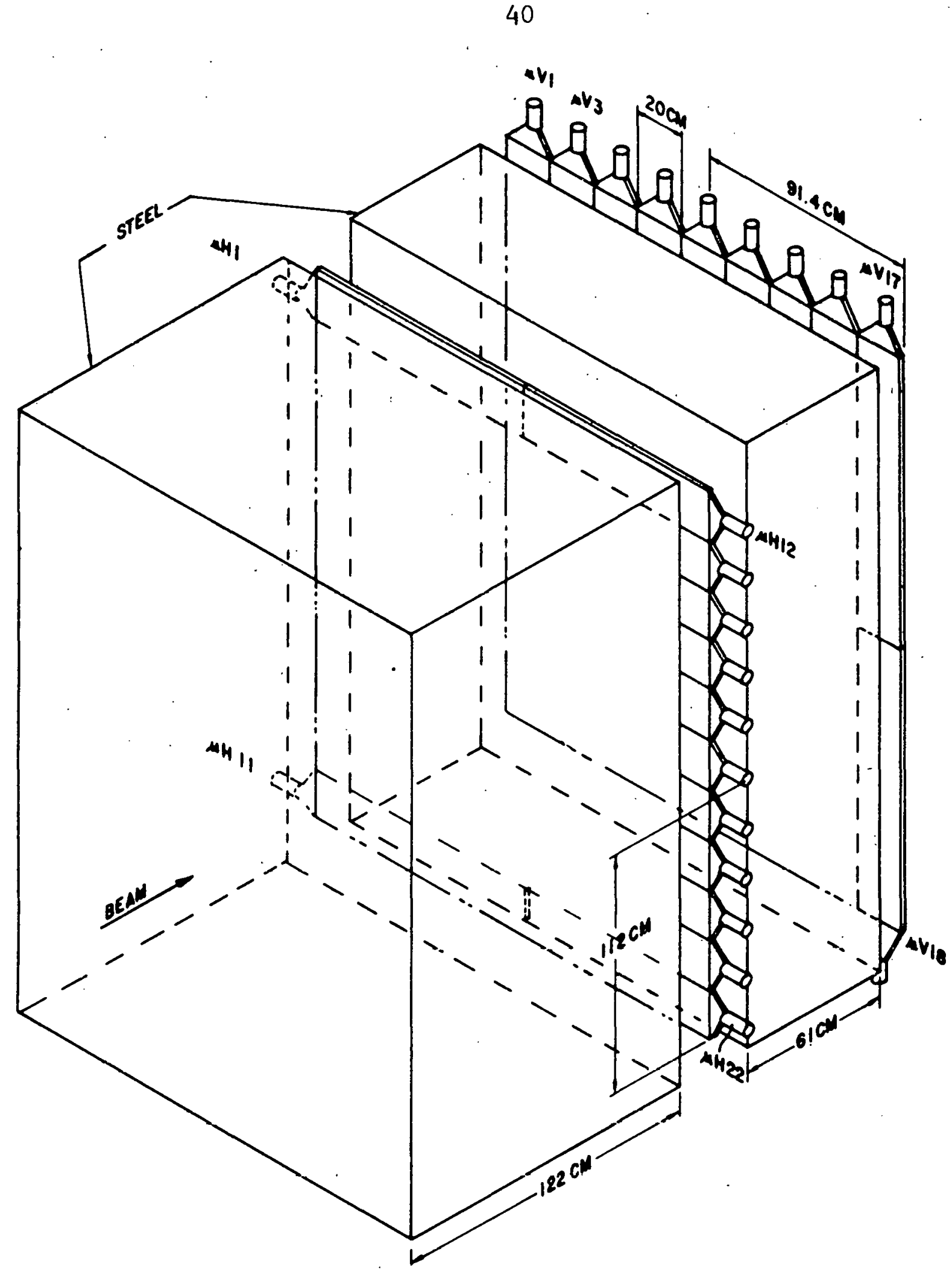

Figure 2.16 

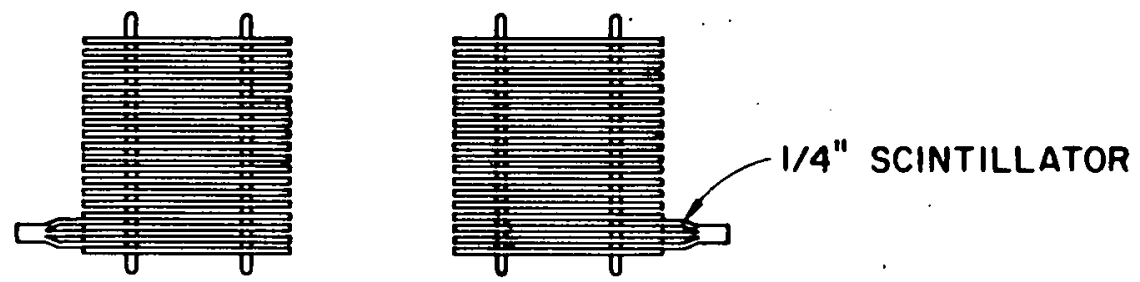

1/4" LEAD SHEETS HANGING FROM 2 STEEL BARS
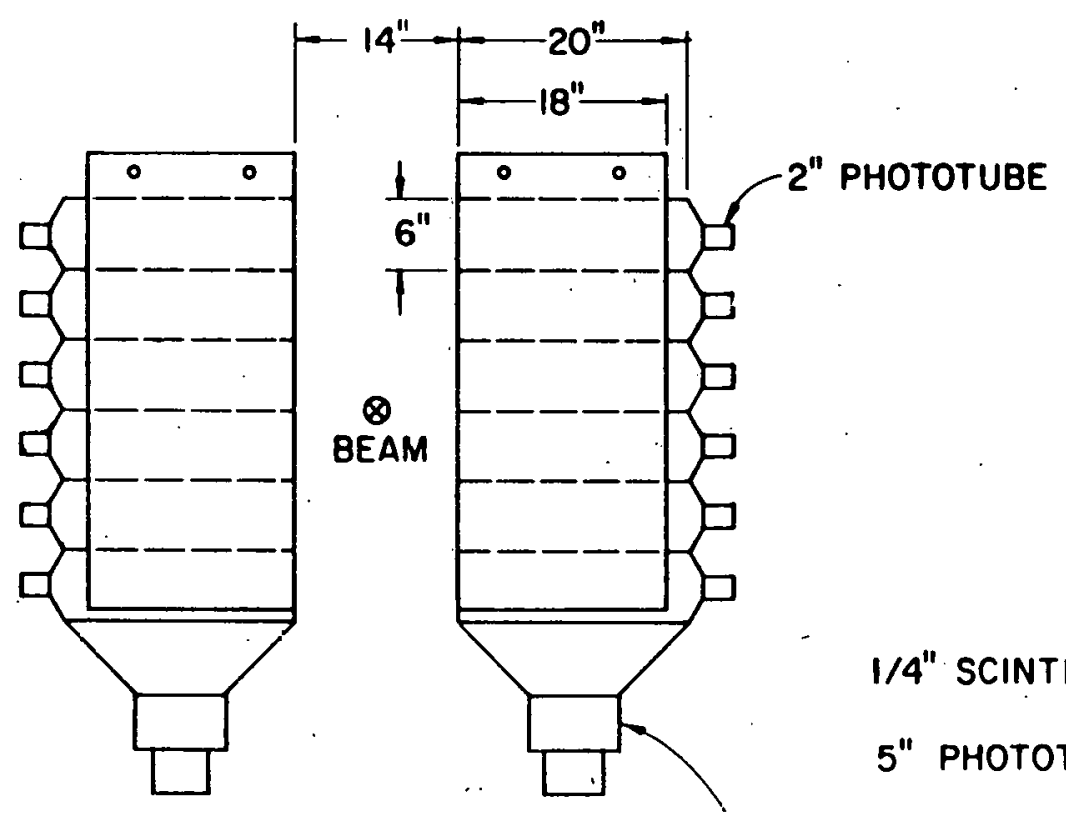

Figure 2.17 
flux from M2 necessitated shielding the 12 front bank counters with .375" wall iron shields and the two back counters with DC-current bucking coils and .75" wall iron shields.

Outer detector muon identification was provided by 8 scintillation counters $(0 \mu)$ located around the M2 aperture on the downstream side thereby using the magnet steel as an absorber.

Section 7: The Trigger

The trigger was comprised of a 2 tiered logic system. A fast master gate (MG) was generated for any event satisfying minimal topological requirements. The slower second level (DC logic), activated for each master gate, made a more. sophisticated trigger decision based on further information from the detector. If an event satisfied the DC logic requirements, an output trigger was generated and the event was recorded.

\section{7a) The Master Gate}

The 2-body inner detector master gate was generated if the pattern of signals from the HxV trigger hodoscope was consistent with 2 tracks in one quadrant and/or 1 track in the two different quadrants. A coincidence with the T-counters was required to ensure that the event candidate was target associated. 
Symbolically, we have:

$$
M G_{I I}=T \cdot H V_{2 \text { BOOY }}
$$

The 2-body outer detector master gate was generated if the pattern of signals from the $\mathrm{HxV}$ hodoscope was consistent with I track in coincidence, with another track at the $\mathrm{OH}$ counters. A coincidence with the T-counters, was also required. Symbolically we have:

$$
M G_{I O}=\mathrm{T} \cdot \mathrm{OH} \cdot \mathrm{HV}_{1 \mathrm{BOSY}}
$$

The HV counters went directly to a fast logic module (figure 2.18) that generated 1 and 2 body outputs. The logical "OR"'s of the 44 T-counters and the 6 OH counters were used for " $\mathrm{T}$ " and "OH" respectively. The $\mathrm{T}, \mathrm{OH}, \mathrm{HV}_{2 \text { Boor }}$ and $\mathrm{HV}_{1 \text { BOOY }}$ signals were input to another fast logic module (figure 2.19) that generated a 2-body master gate if either $M G_{I I}$ or $M_{\text {IO }}$ was satisfied. The master gate was fanned out to a scaler that continualiy monitored the master gate rate (called MG>1 $1_{N D T}$ for "no dead time") and to the confusion logic. The confusion logic module (figure 2.20) gated the DC logic for 200 nanoseconds to allow the higher level logic to settle. 


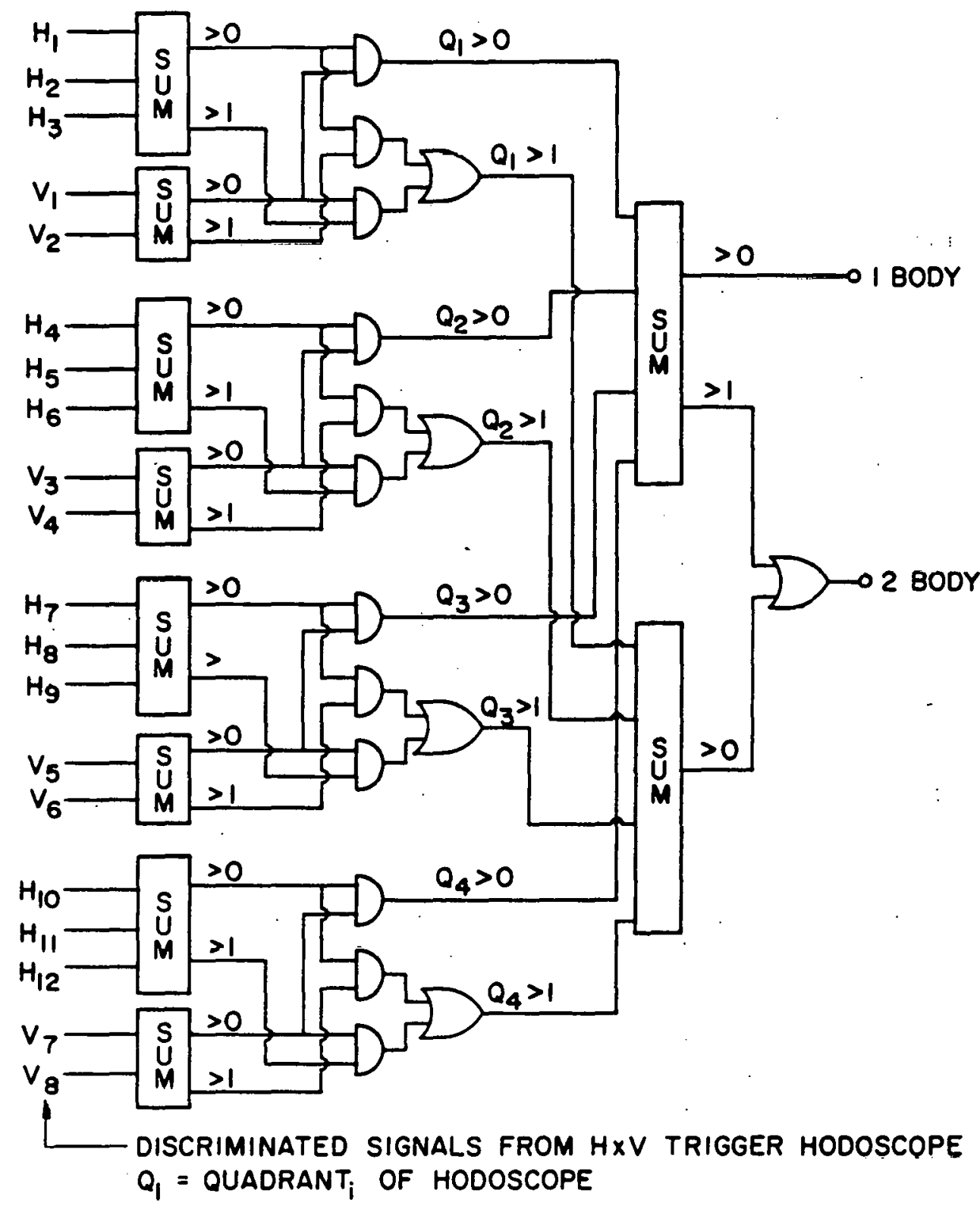

Figure 2.18 


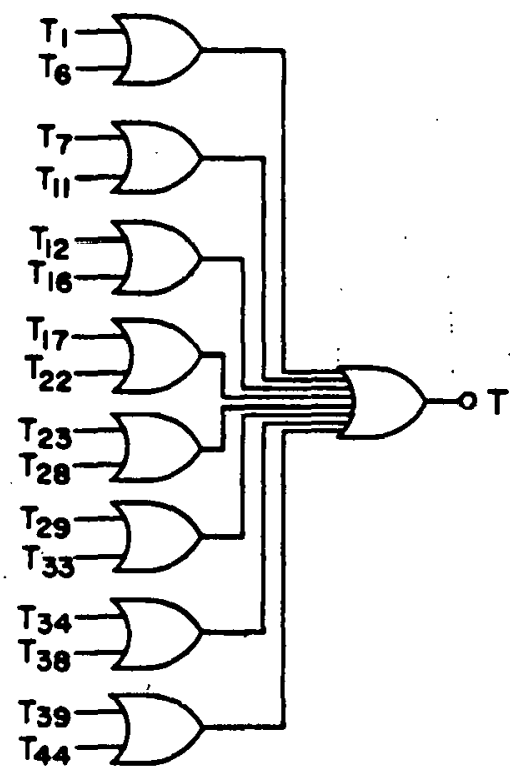

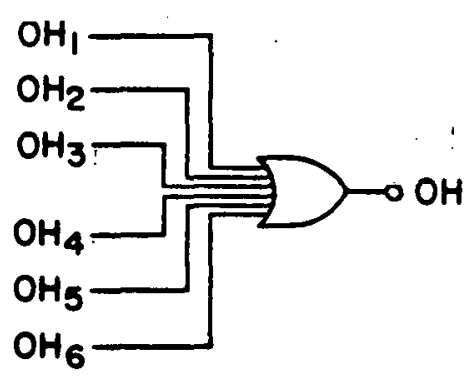

(b)

(a)

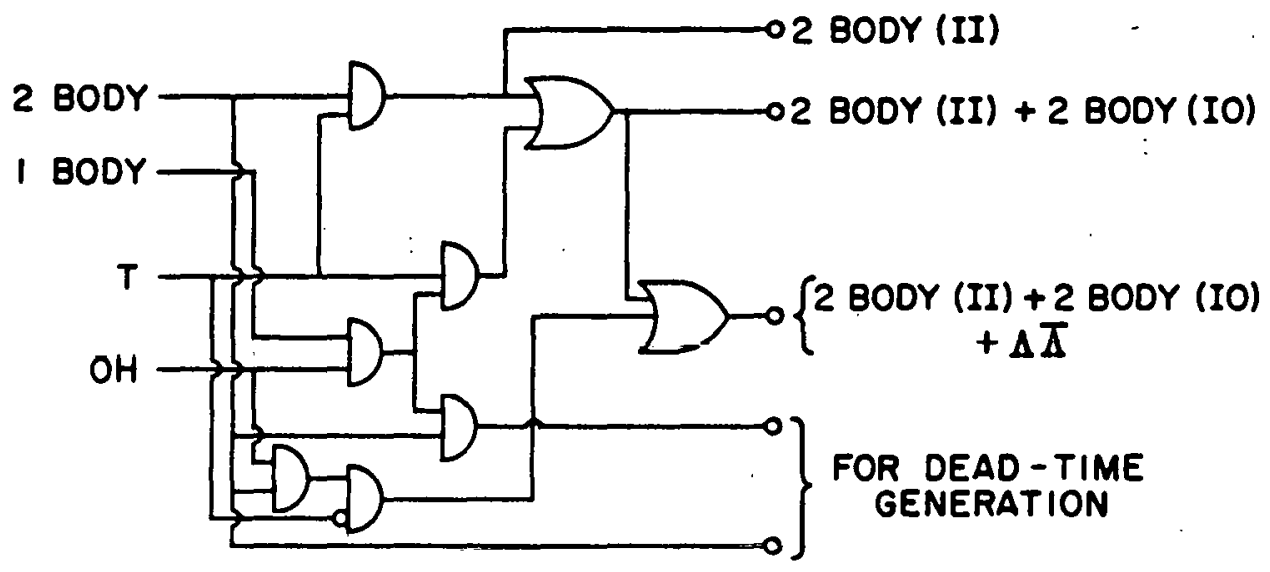

$$
(I I)=\text { INNER }- \text { INNER } \quad(I O)=\text { INNER }- \text { OUTER }
$$

(c) 


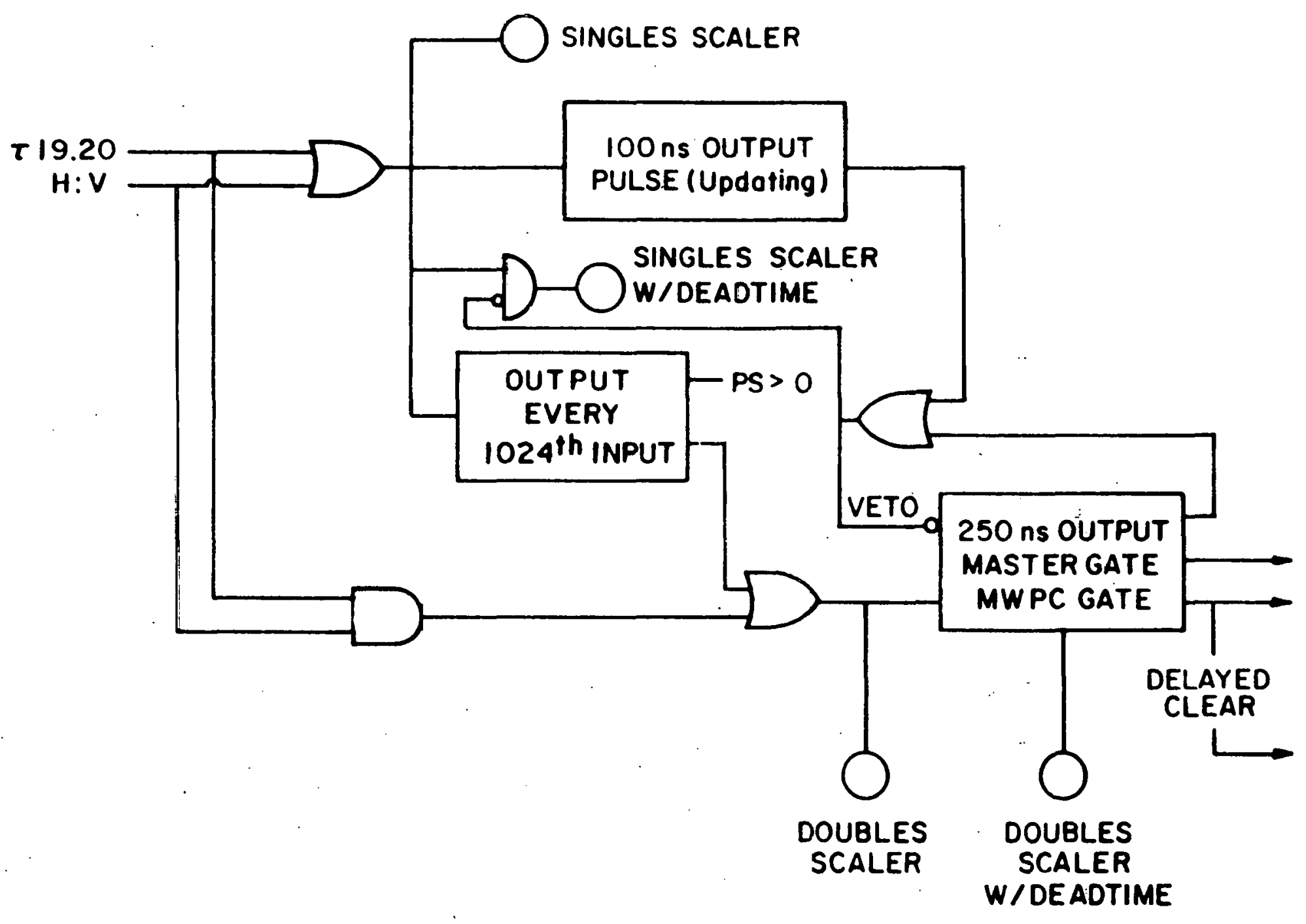


The number of gates to the DC logic from the confusion logic was also recorded by a scaler (called MG $>1_{D T}$ for including "dead time") to monitor the master gate livetime, LT $_{M G^{\prime}}$ :

$$
L T_{M G}=\frac{M G>1_{D T}}{M G>1_{N D T}}
$$

If, after $200 \mathrm{nsec}$, no output trigger was generated, the confusion logic issued a "clear" pulse to the DC logic and waited for the next master gate.

7b) The DC Logic

The DC logic selected events satisfying more complex criteria than those of the master gate. Information from scintillation counters, the MWPC's, the Cerenkov counters, and the hadron calorimeter was grouped together in different logical combinations and encoded as 16 different logic levels, called busslines, by the DC logic. These busslines were "bussed" to logic modules called "pin-logics" (see figure 2.21) that could require, veto, or ignore logical combinations of busslines. The name "pin-logic" comes about because little wire pins are inserted into the modules to hardwire the required logical combinations of busslines. The pin-logics went through prescalers to the output trigger generator. For any event satisfying a pin-logic and its prescale, a signal was sent to the data aquisition system to record the event. The ADC's were gated and an "inhibit" was 
PIN LOGIC

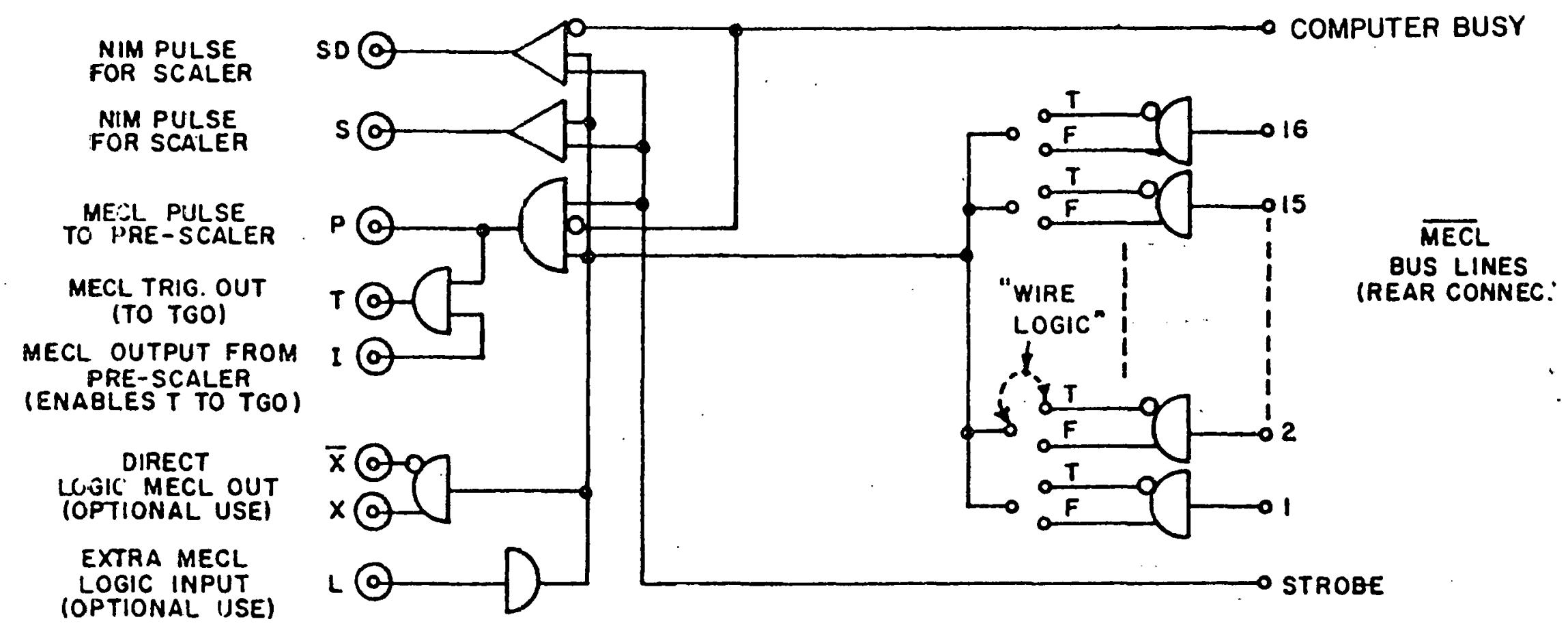


sent to the confusion logic to prevent clearing the data registers before the event was read out.

The data registers (called coincidence registers, or $\mathrm{CR}^{\prime} \mathrm{s}$ ) were double buffered. Upon the generation of an output trigger the CR's were "jam transferred" to the second buffer and held for readout. This released the front-end CR's to continue functioning, forming busslines, and setting pin-logics during event read-out. This allowed the measurement of the DC logic deadtime. Two scalers were output from the pin-logics. One recorded the number of triggers any, time the DC logic was gated by the confusion logic (called Pins for scaler). The other stopped counting triggers whenever an event was being read out (called Pin SD for scaler with deadtime). The master gate was set up as Pin 1 at the DC logic rack to measure the DC logic livetime, ${ }^{L T} T_{C^{\prime}}$ :

$$
L T_{D C}=\frac{\operatorname{PiN} 1 \text { sD }}{\operatorname{PiN} 1_{\mathrm{s}}}
$$

7c) Pin 5

The data analyzed in this thesis were recorded under a 2-track hadronic final state trigger called Pin 5 . Pin 5 satisfied the following logical equation:

$$
\operatorname{Pin} 5=1 \cdot \overline{2} \cdot \overline{7} \cdot 12
$$


where the right hand side refers to bussline numbers. Bussline \#l is the few-body inner detector bussline determined from the MWPC band signals. Bussline $\# 2$ is the multi-body inner detector MWPC bussline. Reference [19] contains a complete description of how the various MWPC busslines were formed from the MWPC band signals. Bussline \#7 is the sum of the beam anti-counters described in section 5c. Bussline \#12 is the hadron calorimeter energy bussline. The dynode signals from the HC were summed and sent to a fast pulse-area-digitizer, the PAD, as illustrated in figure 2.22. If the PAD count was above some preset level, requiring a minimum amount of hadronic energy in the calorimeter, bussline \#12 was set. The PAD was also fanned out and recorded by an $A D C$. This is discussed further in chapter 4.

Section 8: The Data Aquisition System

Data aquisition was handied by the Automatic Control Entry system [18], ACE, anytime an output trigger was generated. ACE was preprogrammed with the addresses for all relevant information from an event. The data were read out and stored in a $64 \mathrm{~K}$ buffer memory at $250 \mu$ sec per event. The buffer was written out to magnetic, tape between beam spills from the main accelerator ring ( 1 every 8 seconds) at 1600 BPI by a PDP-1l on line computer. Figure 2.23 gives an overview of the data aquisition system. 


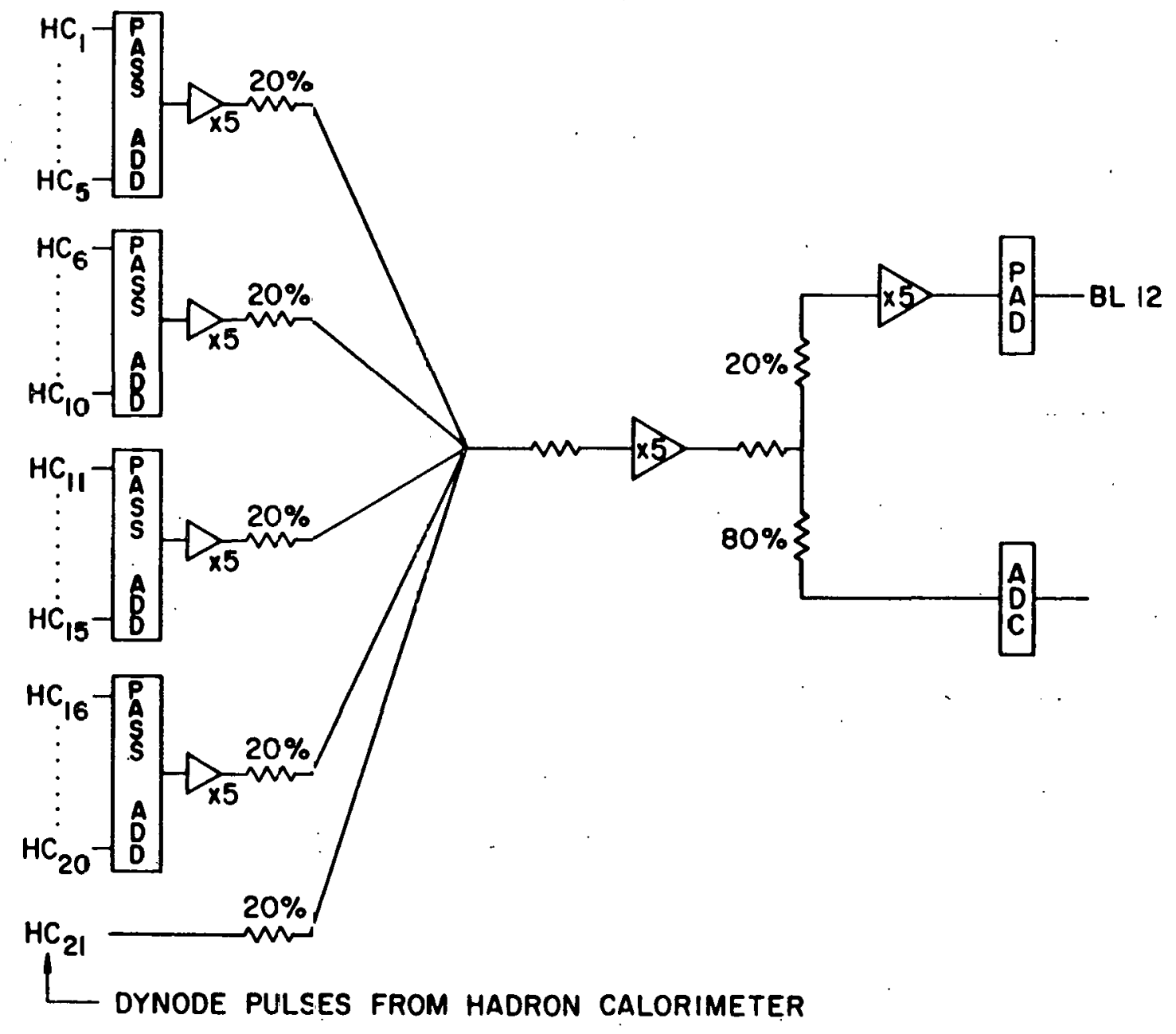


BEAM ON SIGNAL

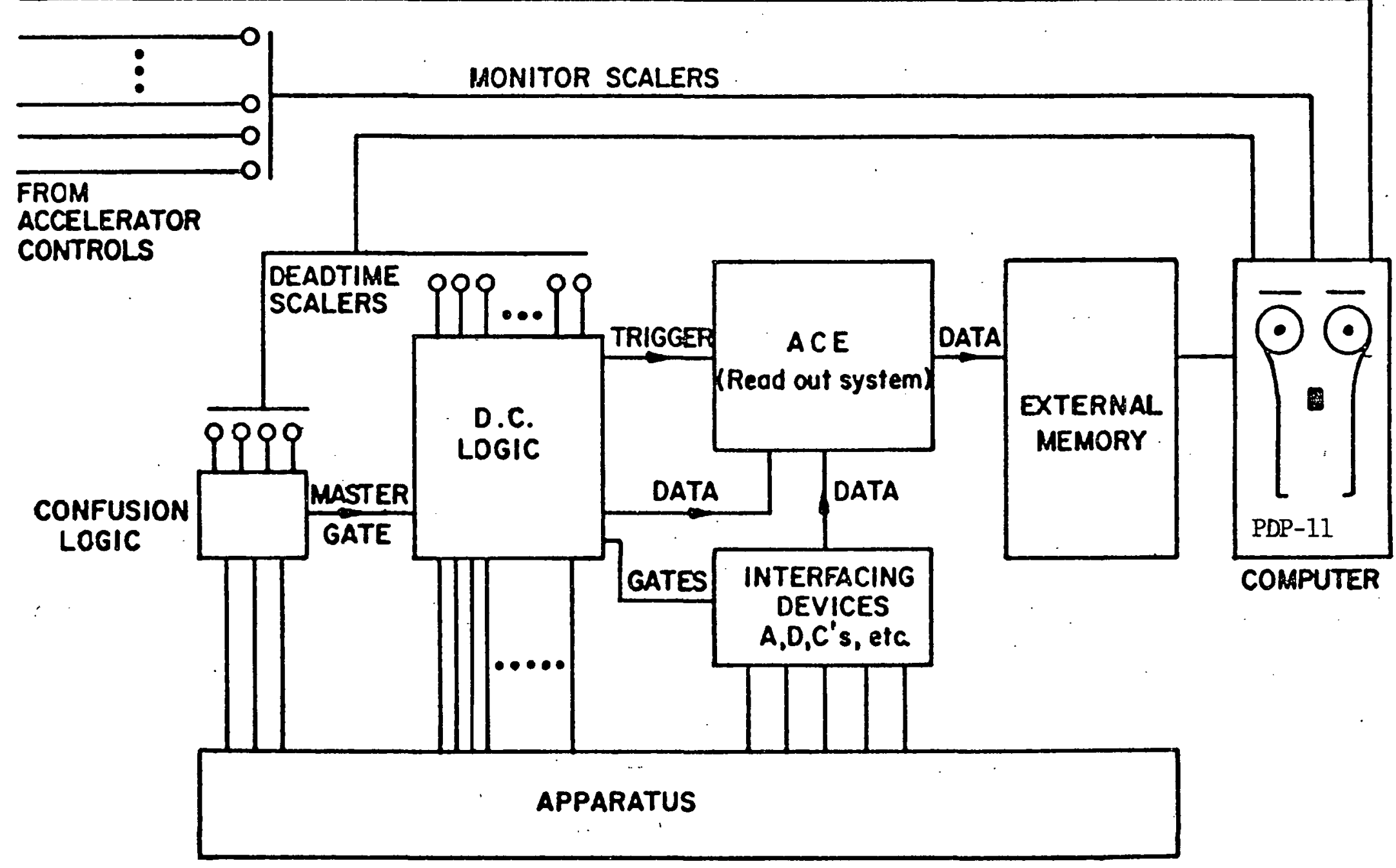

Figure 2.23 


\section{Chapter III}

Reconstruction and Edit for Diffractively produced 2-Track Final states

The data analyzed in this thesis were collected during February, 1979. Approximately 3 million triggers were recorded on 901600 BPI data tapes. $1.1 \times 10^{15}$ protons were delivered on target from the Fermilab accelerator ring yielding $1.7 \times 10^{10}$ photons with an energy $>35 \mathrm{GeV}$ on target from the neutral beam line. Twelve data tapes $(\sim 450,000$ triggers) were recorded with 6 radiation lengths of lead in the beam to attenuate the photon content and measure the background due to hadrons. These runs are referred to as the " $K_{L}^{0}$ runs" in this thesis. Four data tapes were taken with the $\mathrm{LH}_{2}$.target emptied of hydrogen to measure background production from the mylar target vessel. These runs are referred to as the "MT target" runs.

Section 1: Track Reconstruction

A track reconstruction program performed a pattern recognition algorithm on the raw NiWPC wire addresses. A description of this algorithm can be found in $[15,20]$. The program returned the real space track parameters, determined from the fitted tracks, listed on the following page. 
1) The $x$ position at the bend plane of $M 2$

2) $\hat{\mathrm{N}}_{x}$, the $x$ direction cosine

3) The $y$ position at the bend plane of M2

4) $\hat{\mathrm{N}}_{y}$, the $y$ direction cosine

5) $\Delta \hat{N}_{y}$, the bend angle in $M 2$

6) ITYP $=1: 5$ chamber track $P \emptyset-P 4$

$3: 4$ chamber track $P \emptyset-P 3$

$8: 3$ chamber track $P \emptyset-P 2$

(this type does not go

through M2)

The 5 chamber tracks (ITYP $=1$ ) are the best resolved. The resolution for these tracks is $\triangle P / P=2.58$ at $P=100 \mathrm{GeV}$. Only events with 5-chamber tracks were analyzed in this thesis. The track momentum is determined from the bend angle and the field integral of the analyzing magnet. The transverse momentum "kicks" of the magnets during data taking were $.523 \mathrm{GeV} / \mathrm{C}$ for Ml and $.808 \mathrm{GeV} / \mathrm{C}$ for M2.

Section 2: 2 Track Edit

Events analyzed from the reconstructed data tapes were required to satisfy the following criteria:

1) Pin 5: the hadronic trigger

2) 2 5-chamber tracks only (ITYP $=1$ )

3) $Q_{1}+Q_{2}=\emptyset$; two oppositely charged tracks 
4) $\left|\hat{N}_{x_{1}}-\hat{N}_{x_{2}}\right|>.3$ milliradians; $\hat{N}_{x_{i}}=\begin{aligned} & x \text { direction } \\ & \text { cosine of track } i\end{aligned}$

This requirement on the opening angle in the $x-p l a n e$ cuts the copiously produced $e^{+} e^{-}$ pairs produced at $-\emptyset^{\circ}$ opening angle. Figure 3.1 shows the $x$-plane opening angle for 2-track events. Note the $\mathrm{e}^{+} \mathrm{e}^{-}$pairs at zero opening angle.

Events remaining $=229,8 \emptyset \emptyset$

5) $-269.5^{\prime \prime}<z_{V}<-253.5^{\prime \prime}$ : target cut

This required the 2 -track vertex to be in the $\mathrm{LH}_{2}$ target volume. Figure 3.1 shows the vertex distribution at the $\mathrm{LH}_{2}$ target for 2-track events. Events remaining $=155,300$

6) Master gate cut :

Figure 3.2 shows the HxV trigger hodoscope multiplicity for 2-track events satisfying the first 5 requirements. $50 \%$ of the time there are more than 4 counters on. The $\mathrm{HxV}$ counters intercepted by the two measured tracks were required to logically satisfy the master gate independent of any. other hits at the trigger hodoscope. Events remaining $=144,000$

7) AW counters off :

The AW counters, bordering P2, could be triggered by a low energy charged particle in the final state ( $<5 \mathrm{GeV})$ swept out of the MWPC system by Ml. This requirement reduces the probability that any charged tracks in the final state were unobserved. Events remaining $=99,100$

8) 5" fiducial cut at the hadron calorimeter :

Both tracks were required to be more than $5 "$ inside the outside edges of the calorimeter. This minimized leakage of hadronic energy out the sides and therefore facilatated a better understanding of the energy bussline. This is discussed in detail in chapters 4 and 7 . Events remaining $=91,500$ 

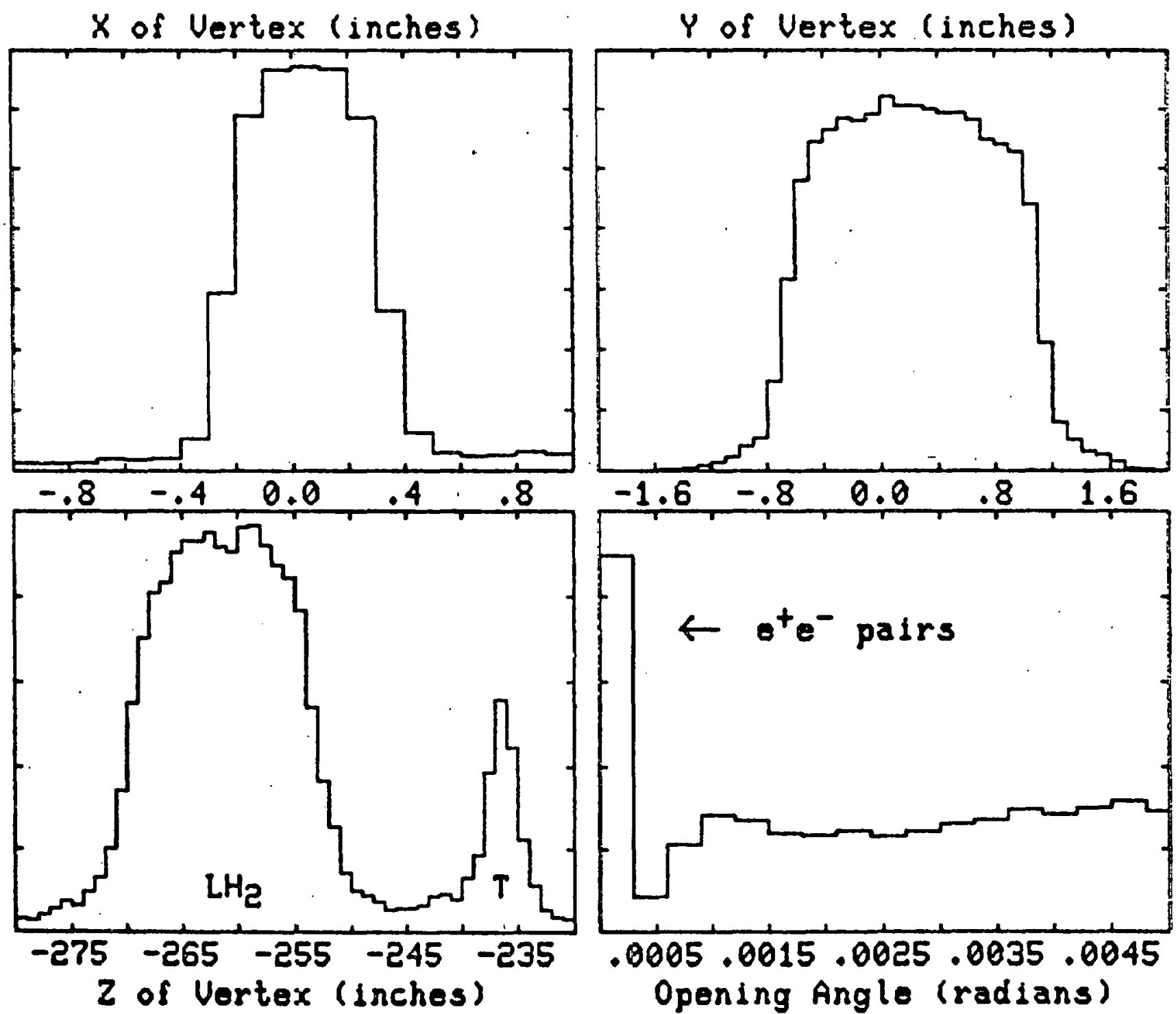

Figure 3.1 


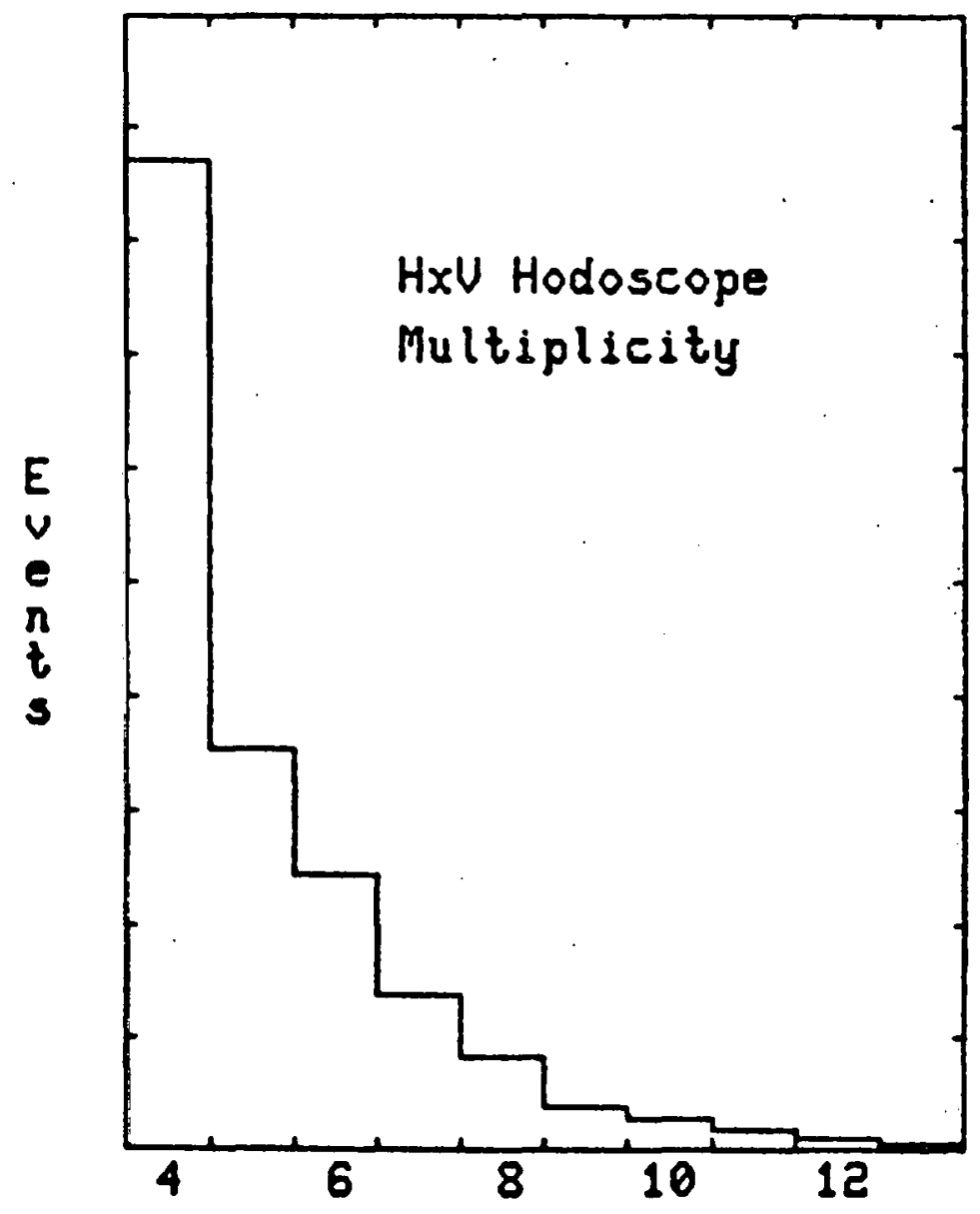

Figure 3.2 
Section 3: The Lead Glass Edit

The shower finding routines for the lead glass array operated independently of any charged track information. A description of the shower finding analysis can be found in [19]. Many of the showers found in the lead glass were produced by hadronic interactions. A shower was considered to be associated with a charged track if the distance of closest approach between them at the lead glass (DCA) satisfied the following criteria:

DCA 5": shower fully contained in large blocks $\mathrm{DCA}<3.5 "$ : shower shared between large and small blocks DCÁ3.5": shower fully contained in small blocks

The energy of the charged track associated showers was used to better determine the remaining hadronic energy entering the calorimeter. This is discussed in detail in chapter 7. Any unassociated showers left atter scanning the charged tracks were called photons. All 2-photon masses were computed for showers greater than $6 \mathrm{GeV}$. Any 2-photon mass within $+/-40 \mathrm{MeV}$ of $135 \mathrm{MeV}$ was called a $\pi^{\circ}$. Any event containing a fully reconstructed $\pi^{\circ}$ was cut from this analysis (events remaining $=84,200$ ). 
Section 4: The Cerenkov Edit

A description of the Cerenkov counter analysis can be found in [16]. The Cerenkov analysis was used loosely as a clean up cut. In looking for the $\phi \rightarrow \mathrm{k}^{+} \mathrm{K}^{-}$, the event was cut if both tracks were unambiguously identified as pions by the Cerenkov analysis. This reduced the reflected low mass. di-pion background under the $\phi$ peak by 208 . Less than 68 of the actual $2 \mathrm{~K}$ events failed to pass this cut. The Monte Carlo (chapter 6) modeled the small inefficiency due to this cut.

In looking at the $\rho \rightarrow \pi^{+} \pi^{-}$, the event was cut if either track was unambiguously identified as a kaon by the cerenkov analysis. This reduced any distortion in the $\rho$ shape due to $\mathrm{K}^{*} \rightarrow \mathrm{K} \dot{\pi}$ backround. Less than $3 \%$ of the actual $2 \pi$ events within $+/-150 \mathrm{MeV}$ of the $\rho$ mass failed to pass this cut. The residual inefficiency of this cut was modeled by the Monte Carlo.

Section 5: The Recoil Detector Ed1t

The analysis of the recoil detector is an essential part of this thesis and is therefore described in detail in chapter 5 and in appendix D. Events that were not consistent with elastic production, as determined by analysis of the recoil detector, were cut from the analysis. $65 \%$ of the events with a di-pion mass within $+/-150 \mathrm{MeV}$ of the $\rho$ mass passed this cut. $50 \%$ of the events with a di-kaon mass within $+/-7 \mathrm{MaV}$ of the $\phi$ mass passed this cut. 


\section{Chapter IV}

Measurement of the Hadron Calorimeter Threshold

The hadronic energy bussline requirement in Pin 5 (chapter 2, section 7c) was "set" based on the sum of the dynode signals from the hadron calorimeter $(\mathrm{HC})$. The sum of the dynode signals was compared to a preset level which would reject events not containing a minimum amount of hadronic energy. This requirement was necessary to reduce the ever-present electromagnetically produced $\mathrm{e}^{+} \mathrm{e}^{-}$backround in photoproduction experiments. Pin 5 therefore contained an energy dependence (other than geometrical) through the hadronic energy bussline.

This thesis presents the energy dependence of the $\rho$ and $\phi$ vector meson cross sections. Understanding the energy bias induced by the HC trigger threshold was therefore crucial to making these measurements. The uncertainty in this understanding is, in fact, the limiting systematic uncertainty in this thesis. Measuring the HC threshold also presented a major obstacle to the completion of this analysis.

Ordinarily, the solution to the problem of understanding the energy bias in Pin 5 would have been to record, simultaneously with $\mathrm{Pin} 5$, an event trigger identical to Pin 5 without the $\mathrm{HC}$ requirement. To monitor a high multiplicity trigger, for example, dropping the hadronic energy bussine requirement would be feasible. For a 2-track final state trigger in a photoproduction experiment, using a monitor 
trigger without the hadronic energy bussline would admit enough electromagnetic backround so that the monitor trigger rate would limit, to an unacceptable extent, the number of real hadronic events recorded.

Being under pressure to fully utilize the available beam time at Fermilab, data taking went ahead recording only $p$ in 5 . The problem of understanding the energy bias due to the HC threshold was deferred for later consideration.

Approximately half way through our data taking run, the comparator setting for the energy bussline was doubled to further reduce the trigger rate due to non-hadronic final states. The analysis of the HC threshold presented in this chapter exploits this change in the calorimeter threshold to measure the energy bussline bias.

In the following discussion, the term "PAD" refers to the sum of the HC dynode signals computed from the anode signals (each recorded individually) and the anode/dynode ratio for each HC module. During data taking, the dynode signals were summed in hardware (see figure 2.22) before they went to the triqqer, and only this sum was recorded. In the off-line analysis it was found that the threshold behavior could be seen more clearly by computing the PAD from the anode signals instead of using the hardware dynode sum. The anode 'signals are typicaily ten times larger than the dynode signals and therefore more accurately represent the hadronic energy in the HC. See appendix B for a detailed discussion about computing 
the PAD for each event.

The data taken before the energy comparator setting was doubled are referred to as the low threshold.(LT) data and the data taken after the comparator setting was doubled are referred to as the high threshold (HT) data. These two data sets are identical in all respects except for the change in the HC threshold. Figure 4.1 shows the PAD distribution for the two different running periods (using identical event cuts).

By normajizing the two running periods to each other (W.R.T. electronic live-time and total beam exposure), and then taking the ratio of these two normalized distributions, the function $f_{R}$ (PAD) representing the $H T$ efficiency, $\epsilon_{H T}(P A D)$, divided by the LT efficiency, $\epsilon_{L T}$ (PAD), was measured. Note: for the rest of this chapter we use the variable $x$ to represent the PAD channel number as the argument of a function.

$$
\mathrm{f}_{R}(\mathrm{x})=.7826 * \mathrm{~N}_{H T}(\mathrm{x}) / \mathrm{N}_{L T}(\mathrm{x})=\epsilon_{H T}(\mathrm{x}) / \epsilon_{\angle T}(\mathrm{x})
$$

where:

$$
\begin{aligned}
\mathrm{N}_{H T}(\mathrm{x})= & \text { \#events in } \mathrm{HT} \text { data set with a } \mathrm{PAD}=\mathrm{x} \\
\mathrm{N}_{L T}(\mathrm{x})= & \text { \#events in LT data set with a PAD }=\mathrm{x} \\
.7826= & \text { Normalization factor between the two } \\
& \text { data sets } \\
\epsilon_{H T}(\mathrm{x})= & \text { Function representing the HT trigger } \\
& \text { efficiency as a function of PAD channel }=\mathrm{x} \\
\epsilon_{L T}(\mathrm{x})= & \text { Funtion representing the LT trigger } \\
& \text { efficiency as a function of PAD channel }=\mathrm{x}
\end{aligned}
$$




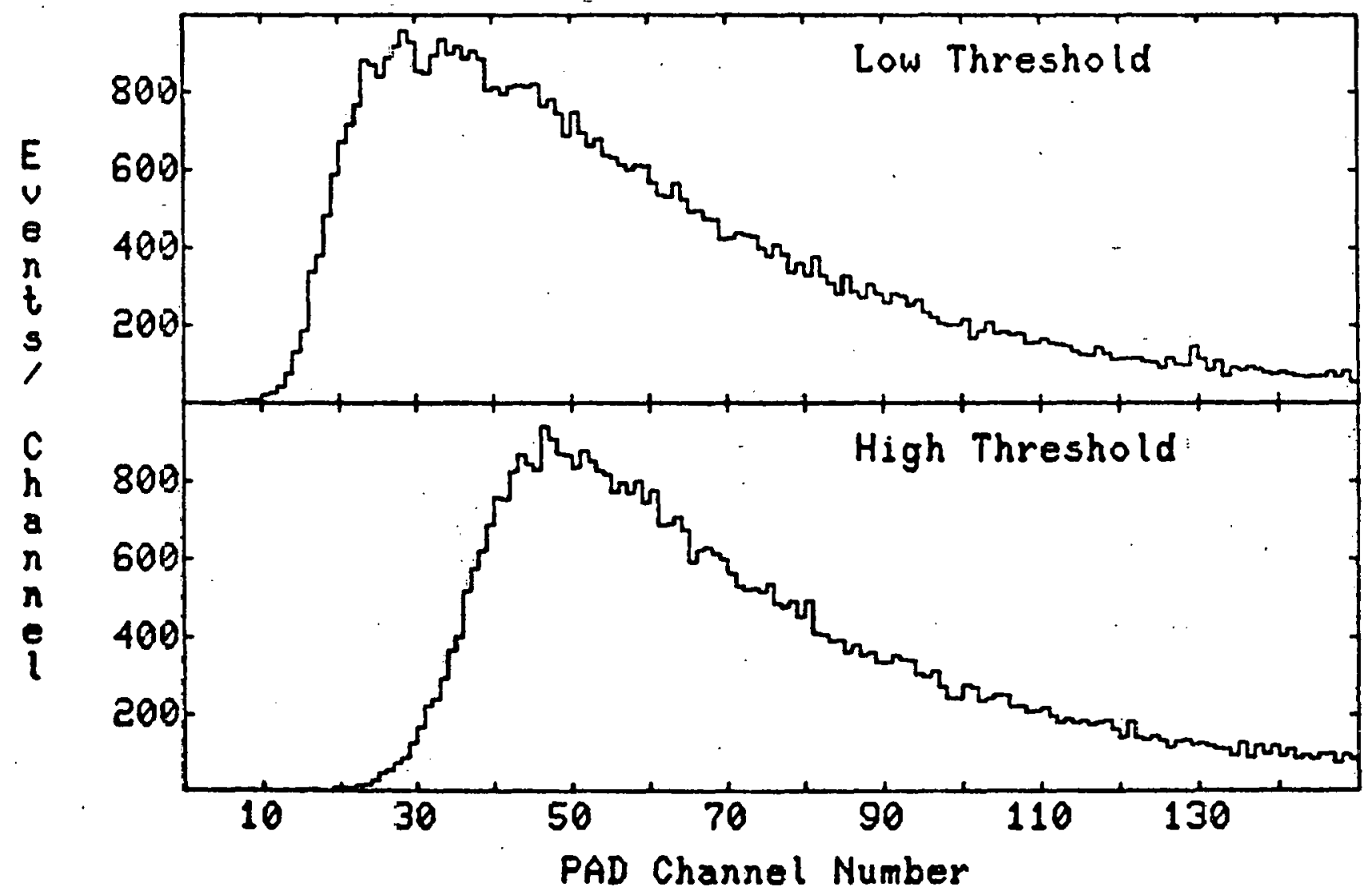



Figure 4.1 
Figure 4.2 shows a fit to $f_{R}$ (PAD) using the intergral of a Gaussian as the fitting function. The best fit obtained a threshold point of 37.5 PAD channels and a width of 6.1 channels.

For values of the $\mathrm{PAD}$ such that $\epsilon_{L T} \approx 1, f_{R}$ is equal to the HT efficiency function $\epsilon_{H T}$. The LT point is approximately a factor of two lower ( channel \#19) than the HT point. In the region where $f_{R}$ is non-zero $\left(\epsilon_{H T}>\emptyset\right)$, $\epsilon_{L T} \approx 1$. The measurement of $E_{R}$ is therefore equivalent to a measurement of $\epsilon_{H T}$. This function $\epsilon_{H T}$ (PAD) is the oniy measurement available to determine the energy dependence of Pin 5. Unfortunately, $\epsilon_{H T}$ measures the bussline efficiency in terms of the PAD channel number and not in terms of the energy.

The problem next becomes understanding how the PAD signal from the $\mathrm{HC}$ is related to the total hadronic energy in the event. The resolution of the $\mathrm{HC}$ is so poor (40\% a.t $50 \mathrm{GeV})$ that understanding the relationship between pulse height and energy deposition is an inherently difficult problem. This problem is further complicated by two other factors. First, the location of the lead glass array and the back shower counters immediately upstream of the HC place $\sim 2$ interaction lengths of material in front of the HC. Even a very high energy hadron has a chance of interacting in this material and losing a substantial part of its energy, particularly if a $\pi^{\circ}$ is produced, before entering the calorimeter. Second, all 


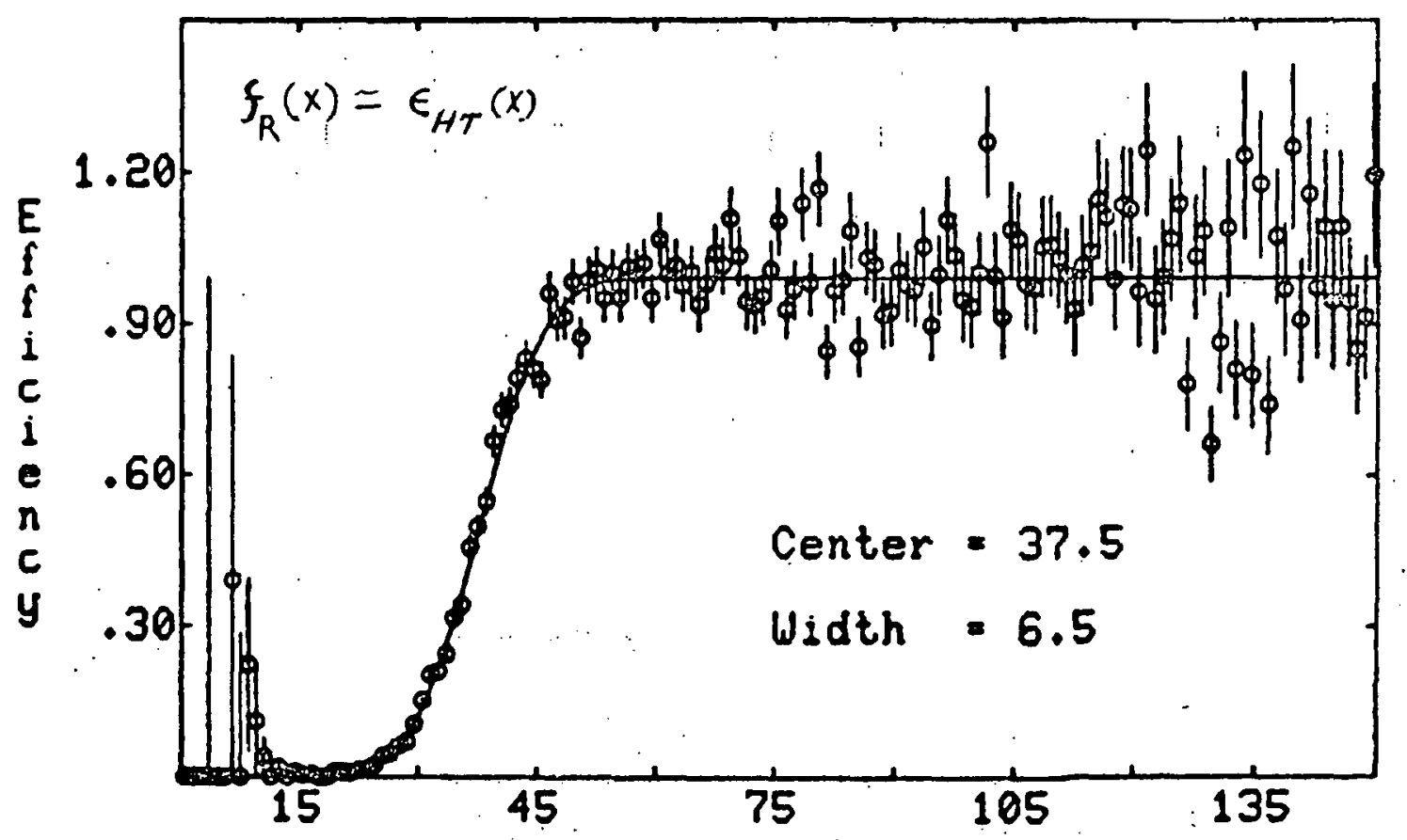

PAD Channel Number

Figure 4.2 
data recorded required the $\mathrm{HC}$ energy bussline. No unbiased events were recorded. Unbiased events would be required to study pulse height variation versus energy deposition and to develop a detailed Monte Carlo which could, ideally, predict a PAD for a generated event. A simulated PAD could then be tested against $\epsilon_{H T}$ to measure the acceptance.

The solution to the problem was based on a very simple idea. By definition, the acceptance (A) for any final state is the number of observed events $\left(\mathrm{N}_{0}\right)$ divided by the number of "true events". $\left(\mathrm{N}_{T}\right)$ :

$$
A=N_{0} / N_{T}
$$

The fact that the $50 \%$ efficiency point in $\epsilon_{H T}$ is channel 37 implies that the number of observed events with a PAD of 37 represents one half of the true number of events with a PAD of 37 . Or, in general,

$$
N_{T}(x)=N_{0}(x) / \epsilon_{H T}(x)
$$

The acceptance in an energy region $E_{1}-E_{2}$ (or any variable, for that matter) can be computed by plotting the PAD distribution for events in that energy window and dividing the PAD distribution by $\epsilon_{H T}$. The PAD distribution will still be very broad because of the resolution and energy loss problems mentioned before. However, the acceptance can be ascertained 
by integrating the above equation over the entire PAD distribution:

$$
A(E)=\frac{\sum_{\substack{C H A N N E L \\ N U M B E R}} N_{0}(X)}{\sum_{\substack{C, H A N N E L \\ N U M B E R}}\left[N_{0}(X) / \epsilon_{H T}(X)\right]} ; E_{1}<E<E_{2}
$$

Figure 4.3 illustrates this technique. The observed PAD distribution and the computed "true" PAD distribution, $x / \epsilon_{H T}(x)$, are plotted for the $e$ data. The ratio of the areas under the two curves in figure 4.3 represents the total acceptance for the $\rho$ due to the calorimeter threshold.

The virtues of this technique are many. It is an inherently simple and direct use of the only measurement of the calorimeter threshold, even though this threshold function is a function of PAD channel number and not an explicit function of energy. The energy dependence is unfolded simply by looking at the PAD distribution in different energy regions and computing $A(E)$. Using this technique for computing the acceptance by-passes completely the difficult problem of developing a Monte Carlo to simulate hadron shower development in the HC. The effects of energy loss in the material preceeding the calorimeter are folded in automatically, along with any other extraneous problems such as having one or two dead phototubes during data taking. 


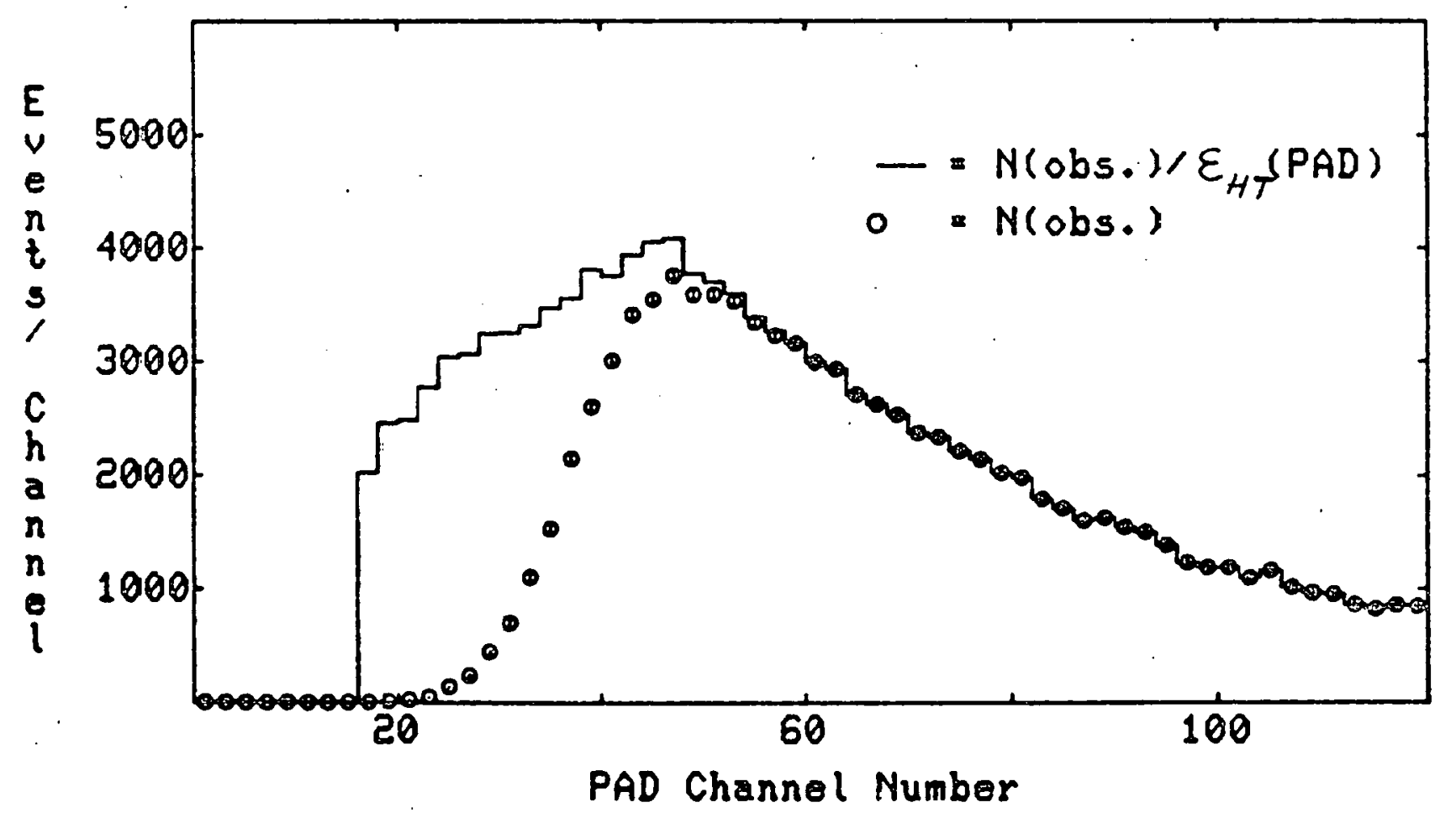

$\stackrel{\infty}{\infty}$

Figure 4.3 
There are two limiting situations for using this technique. First, nothing can be said about the number of "true" events that have a PAD value such that $\epsilon_{H T}=0$, since none, or essentially none, will be observed $\therefore$ In the region where $\epsilon_{H T}$ is small, but non-zero, only a small fraction of the true events are observed. Dividing the observed number of events by this small fraction results in a large systematic uncertainty in the acceptance calculation. The accuracy of the $\rho$ and $\phi$ analyses at lower energies, where more and more of the events leave small pulse heights in the $\mathrm{HC}$, is limited by the uncertainty in the acceptance. No attempt has been made to analyze $\rho$ and $\phi$ events with an energy less than 35 $\mathrm{GeV}$, where the systematic uncertainty in the acceptance has reached $30 \%-40 \%$. The second limitation is statistical. There must be a sufficiently large number of observed events to detine the PAD distribution well enough so that dividing it by $\epsilon_{H T}$ is meaningful and reasonably well defined.

Fortunately the analyses of the $\rho$ and $\phi$ are not over 1 y constrained by these considerations. There are enough $\rho$ data to define the PAD distribution in several different energy regions and compute the acceptance. There are enough $\phi$ data to determine that the calorimeter acceptance for the $\phi$ is consistent with the calorimeter acceptance measured with the e data. 
The low threshold data present another problem. The low threshold function $\epsilon_{L T}$ is not known, other than that it is $\approx$ I for $P A D$ values where the high threshold is non-zero. This one fact allowed another approach to be taken, however. The LT data were analyzed the same way as the HT data atter first requiring each LT event to satisfy a software "trigger" equivalent to $\epsilon_{H T}$. Including LT events that satisfied a software trigger equivalent to $\epsilon_{H T}$ in the analysis, created, in effect, one large data set as if it had all been recorded subject to the high calorimeter threshold requirement. This approach allowed recovery of $70 \%$ of the LT data for analysis. 


\section{Chapter $\mathrm{V}$}

\section{Analysis of the Recoil Detector}

Use of the recoil protion detector plays a central part in the analysis of the $\rho$ and $\phi$ vector mesons in this thesis. The optical theorem (eq. 1.4) relates the total cross section to the elastic cross section at $t=\emptyset \mathrm{GeV}^{2} / \mathrm{c}^{2}$. The recoil detector is used to select events that are consistent with elastic production assuming the two downstream tracks are-the decay products. Using the recoil detector eliminates three types of contamination to elastic $\rho$ and $\phi$ data.

First, a higher multiplicity final state could pass the diffractive event cuts (chapter 3 ) if the extra particles were unobserved by the downstream spectrometer. Extra particles in the final state could be unobserved if one or more of the decay products was a neutral particle or was a very low momentum track $(<5 \mathrm{GeV})$ and was swept out of the spectrometer by the first magnet (MI). The target nucleon recoiling against a higher multiplicity final state would not be coplanar with the two observed downstream tracks.

Second, if excitation of the target nucleon occurred:

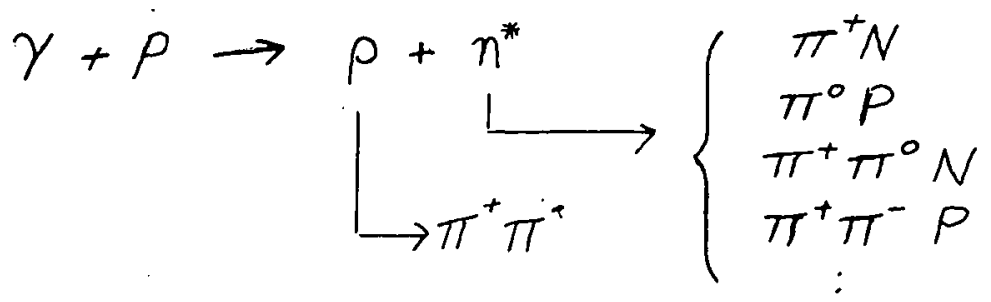

the subsequent $n^{*}$. decay could produce pions that enter the 
recoil detector. A pion would have different range-energy loss characteristics in the recoil detector scintillation counters than a proton. The transverse momentum of a pion could also violate coplanarity with the transverse momentum of the downstream tracks.

Third, the photon beam is contaminated with neutrons and $K_{L}^{0}$ mesons. This fact is particularly important for the $\phi$ analysis. A major background to elastic $\phi$ photoproduction is $\phi$ production from a $\mathrm{K}_{\mathrm{L}}^{0}$ :

$$
\begin{aligned}
& K_{L}^{0}+P \rightarrow \phi+K_{s}^{0}+P
\end{aligned}
$$

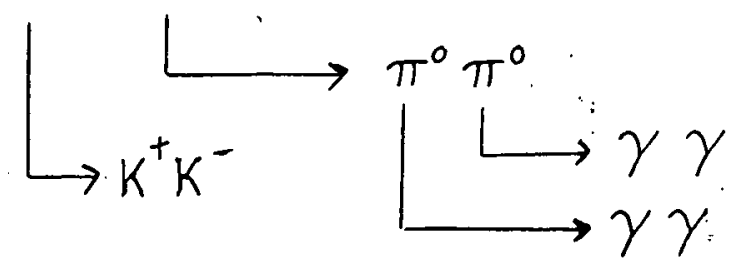

The other neutral particles in the final state could very weli be unobserved, By analyzing the recoil proton, wnich is coplanar to the $\phi K_{S}^{0}$ pair and not to the $\phi$, events produced by $K_{L}^{0}$ 's can be differentiated from photoproduced events and eliminated from the data sample. Without the recoil detector anaysis $30 \%$ of the $\phi$ signal would be $K_{L}^{0}$ induced. Correcting for this $K_{L}^{0}$ induced backqround would necessitate making a major subtraction requiring $K_{L}^{0}$ data of a sufficient quantity in each energy region of interest to make the subtraction meaningful. The errors from the subtraction would still be large. 
Thus, the use of the recoil detector analysis allows the selection of elastically produced events. The t-distribution for these events can be extrapolated to $t=0 \mathrm{Gev}^{2} / \mathrm{c}^{2}$ and the optical theorem applied to extract the total meson-nucleon cross section. The selection of elastic events cleans up the $\rho$ data and eliminates a major background to the photoproduced $\phi$ data.

There were four main stages in the development of the recoil detector analysis program, $\mathrm{RC} 6$.

1) The determination of the recoil detector geometry with respect to MWPC coordinate system of the downstream spectrometer.

2). Modeling the effects of the $\mathrm{LH}_{2}$ target materials (figure 2.4) on the range-energy characteristics of the recoil proton.

3) Determination of how the position and energy resolution of the downstream spectrometer affect the sensitivity of the recoil detector analysis.

4) Classification of events, based on the analysis of the signals in the recoil detector, into distinct event types. The final classification is into one of three categories:

i) Consistent with elastic

ii) Inelastic

i.i.i) Don't know 
Section 1: Geometry

There are two reasons why determining the geometry of the recoil detector is a non-trivial problem. First, the recoil detector $(R C)$ is suspended around the $\mathrm{LH}_{2}$ target and is essentially insensitive to the position of the downstream spectrometer. Tracks measured with the MWPC system cannot be projected to the RC to see where they intersect the counters. Second, the $\mathrm{LH}_{2}$ target is 4 " in diameter and the photon beam spot is 1 " $x 2$ ". The photon beam spot can be moved anywhere within the 12.5 in. $^{2}$ target cross section without attecting the downstream spectrometer. Only if the target is moved out of the beam spot all together will the change be seen because the trigger rates would drop. However, where the beam spot is with respect to the $\mathrm{LH}_{2}$ target does affect the recoil detector analysis because it varies the amount of $\mathrm{LH}_{2}$ that a proton will have to penetrate to exit the target from one side or the other.

From the kinematics for elastic scattering an expression for the 4-momentum transfer squared ( $t)$ is obtained:

$$
-t=\frac{M_{v}^{2}-2 E_{v}\left(E_{v}-\hat{N}_{y} \cdot \vec{P}_{v}\right)}{1-\frac{1}{M_{p}}\left(E_{v}-\hat{N}_{y} \cdot \vec{P}_{v}\right)}
$$


where

$$
\begin{aligned}
& \mathrm{M}_{V}: \text { Vector meson mass } \\
&\left(\mathrm{E}_{V}, \overrightarrow{\mathrm{P}}_{V}\right): \begin{array}{l}
\text { Vector meson energy } \\
\text { and momentum }
\end{array} \\
& \mathrm{M}_{P}: \text { Proton mass } \\
& \hat{\mathrm{N}}_{\gamma}: \begin{array}{l}
\text { Direction cosine for incident } \\
\text { photon }
\end{array}
\end{aligned}
$$

It is known from the measurement of $e^{+} e^{-}$pairs in the spectrometer that the photon beam has a net angular displacement with respect to the MWPC coordinate system.

$$
\widehat{N}_{\gamma}=. \emptyset \emptyset 02 \hat{x}-.00021 \hat{y} \text { radians }
$$

By measuring the momentum of the downstream tracks and assuming a mass for the meson produced, $t$ is determined for the event. The kinematics for elastic scattering also determine the reco'il proton kinetic energy:

$$
T=t / 2 M
$$

Thus, measuring $t$ and requiring conservation of momentum determine the magnitude and direction of the recoil proton momentum with respect to the beam axis.

To determine the counter positions, a sample of events with. 2 downstream tracks and a 2-pion mass within $+/-150 \mathrm{MeV}$ of the $\rho$ mass was chosen. A cut was made requiring that $t$ be large enough $\left(t \gtrsim .15 \mathrm{Gev}^{2} / \mathrm{c}^{2}\right)$ for the proton to unambiguously exit the $\mathrm{LH}_{2}$ target and trigger the $\overline{\mathrm{KC}}$. 
A projection along the momentum vector of the proton was made to the plane assumed to contain the counters of the RC. By rotating to a coodinate system in the plane of the counters the variable $d$, the distance from the counter edge, was determined (figure 5.1). By scanning the variable $d$ for all sides of the RC and plotting against $d$ the efticiency for a particular counter to be "on" the counter edges were determined. These edges are broad due to multiple scattering and resolution effects (section 3 ) but by overplotting all of the counters on one side together, unique boundaries were determined (figure 5.2). Since the event vertex is calculated using the chamber coordinates, these are the counter positions with respect to the MWPC system. The location of the counters with respect to the $\mathrm{LH}_{z}$ target and beam spot was still undetermined.

Next the target center and beam spot coodinates were determined. Figure 5.3 shows how a 2 " $x 2$ "beam spot could be arbitrarily located in the $\mathrm{LH}_{2}$ target. It also shows how the $\mathrm{LH}_{2}$ target could be arbitrarily located with respect to the MWPC coordinate system.

The displacement from the MWPC system to the beam spot center $\left(\vec{x}_{C B}\right)$ was easily determined. Histogramming event vertices at the target showed the beam profile clearly (figure 3.1 ). The centers of this profile in $x$ and $y$ are the 


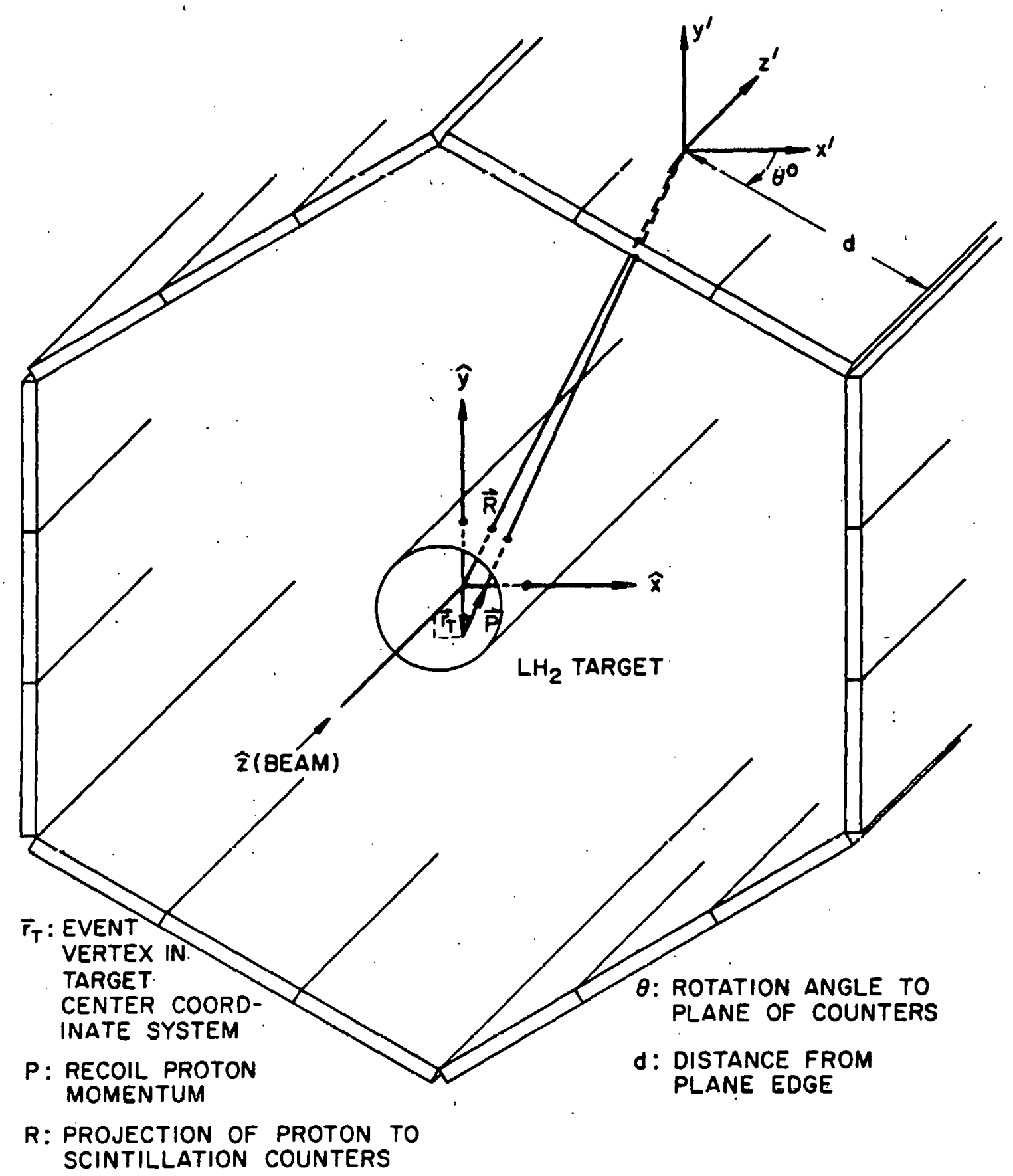

Figure 5.1 


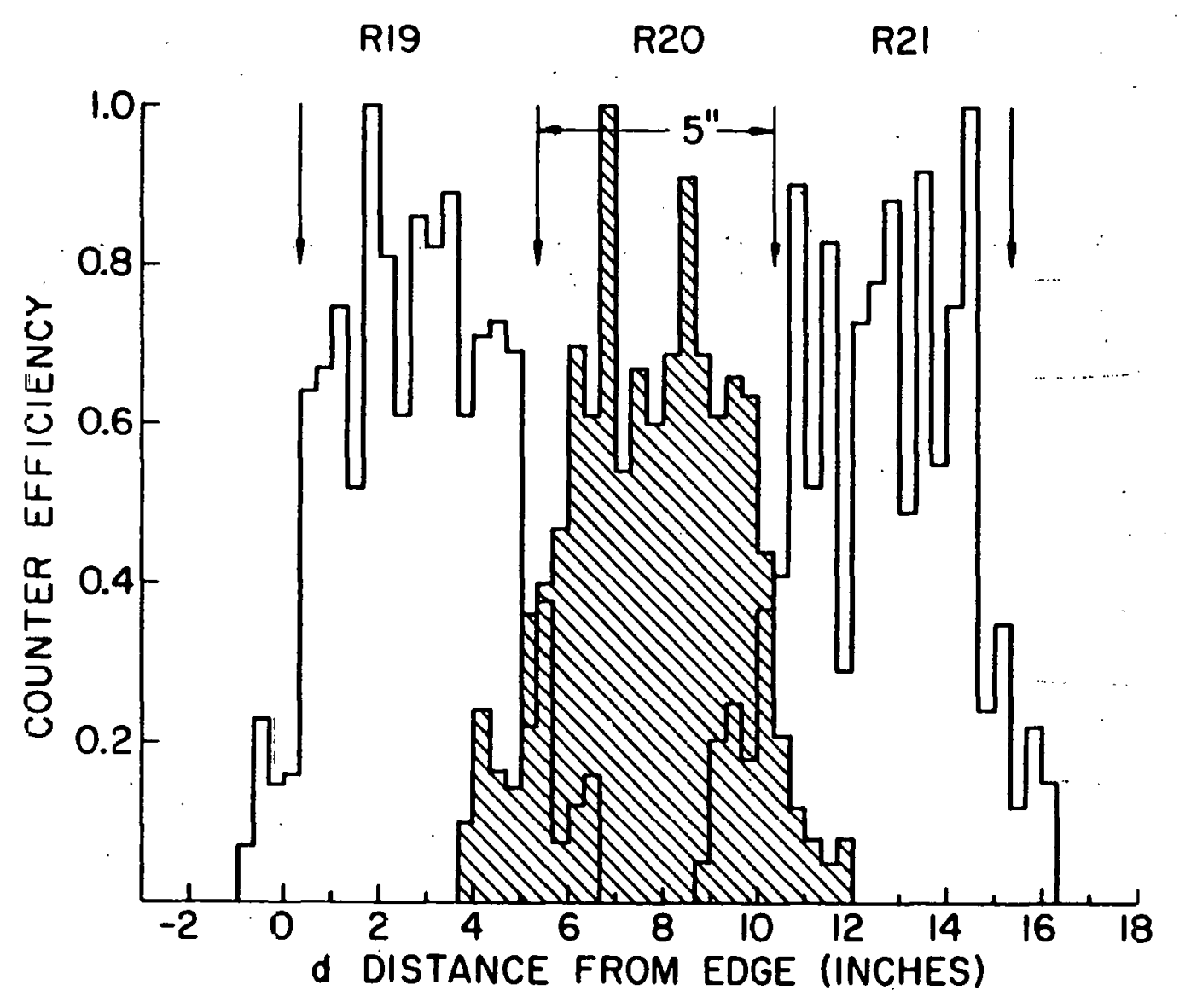




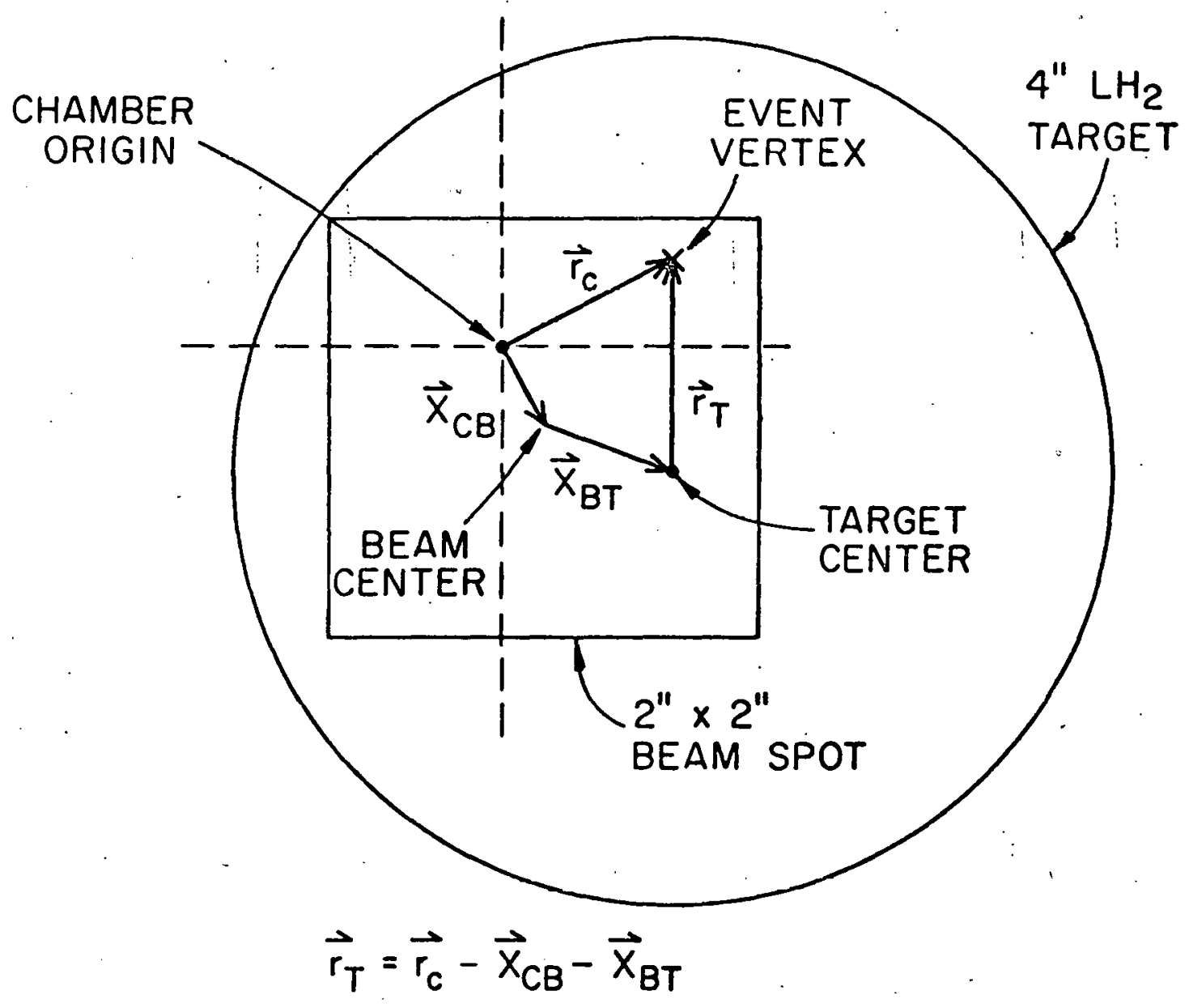

WHERE :

$$
\begin{aligned}
\vec{r}_{c}= & \text { CHAMBER COORDINATES FOR EVENT } \\
\vec{r}_{T}= & \text { TARGET COOROINATES } \\
\vec{x}_{C B}= & \text { VECTOR FROM CHAMBER ORIGIN TO } \\
& \text { BEAM CENTER. } \\
\vec{x}_{B T}= & \text { VECTOR FROM BEAM CENTER TO } \\
& \text { TARGET CENTER }
\end{aligned}
$$


required offsets. It was found that:

$$
\vec{x}_{C B}=.10 " \hat{x}+.22 " \hat{y}
$$

Determining the displacement of the beam spot with respect to the $\mathrm{LH}_{2}$ target center $\left(\vec{x}_{B T}\right.$ in figure 5.3) was more difficult. The program RC6 accurately computes the range of a recoil proton, with a given $t$, through the target materials $\left(\mathrm{LH}_{2}\right.$, mylar, Al-mylar insulation, Al casing; see figure 2.4). Therefore, if the true beam spot location were known $\left(\vec{x}_{B T}\right)$, a prediction could be made by RC6 as to whether or not a recoil proton with a given $t$ was energetic enough to exit the target and trigger the appropriate RC counter. If, for example, the beam spot was centered, the measured counting rates for counters on opposite sides of the $\mathrm{LH}_{2}$ target (figure 2.5A), for a given $t$, would be approximately equal. If one then assumed a centered beam spot for $\vec{x}_{B T}$ in RC6, a prediction of symmetric counting rates would also be made. Conversely, if the beam spot was off to one side of the $\mathrm{LH}_{2}$ target, the counting rate, for a given $t$, would be much higher for the counter on the near side than for the counter on the far side. Likewise, if one assumed a beam spot off-center in RC6, the same asymmetric counting rate woulc be predicted. The solution then was to try a series of hypothetical beam spot positions $\left(\vec{x}_{B T}\right)$ and choose the one where the observed counting rates for all counters best matched the predicted counting rates from $\mathrm{RC} 6$. Although the absolute counting rate depends 
on the assumed t-distribution, the relative asymmetry on opposite sides of the RC does not.

The inner ring contains three pairs of opposing counters. One pair of opposing counters was analyzed at a time. A plane between these two counters was chosen as the beam center so that the best match between the predicted and the observed counting rates was obtained. After balancing all three pairs of counters this way, the intersection of the three planes uniquely defined a region in the target as the best region for the beam center $\left(\vec{x}_{B T}\right)$. Figure. 5.4 shows this solution in detail. It was found that:

$$
\overrightarrow{\mathrm{x}}_{B T}=-.35 " \hat{\mathrm{x}}+.1 \mathrm{i} \| \hat{\mathrm{y}}
$$

- Figure 5.5 shows that the resulting predicted threshold and observed threshold for protons exiting the target agree extremely well. A fit to the threshold point (the value of the abscissa at the halfway point between the minimum and maximum asymptotes) for each was done and agreement was found to within

$$
\Delta t_{\text {THRESHOLD }} \sim .004 \mathrm{GeV}^{2} / \mathrm{c}^{2}
$$

which, in position space in $\mathrm{LH}_{2}$, corresponds to .25 inches:

$$
\Delta\left|\overrightarrow{\mathrm{x}}_{B T}\right| \sim 1 / 4
$$




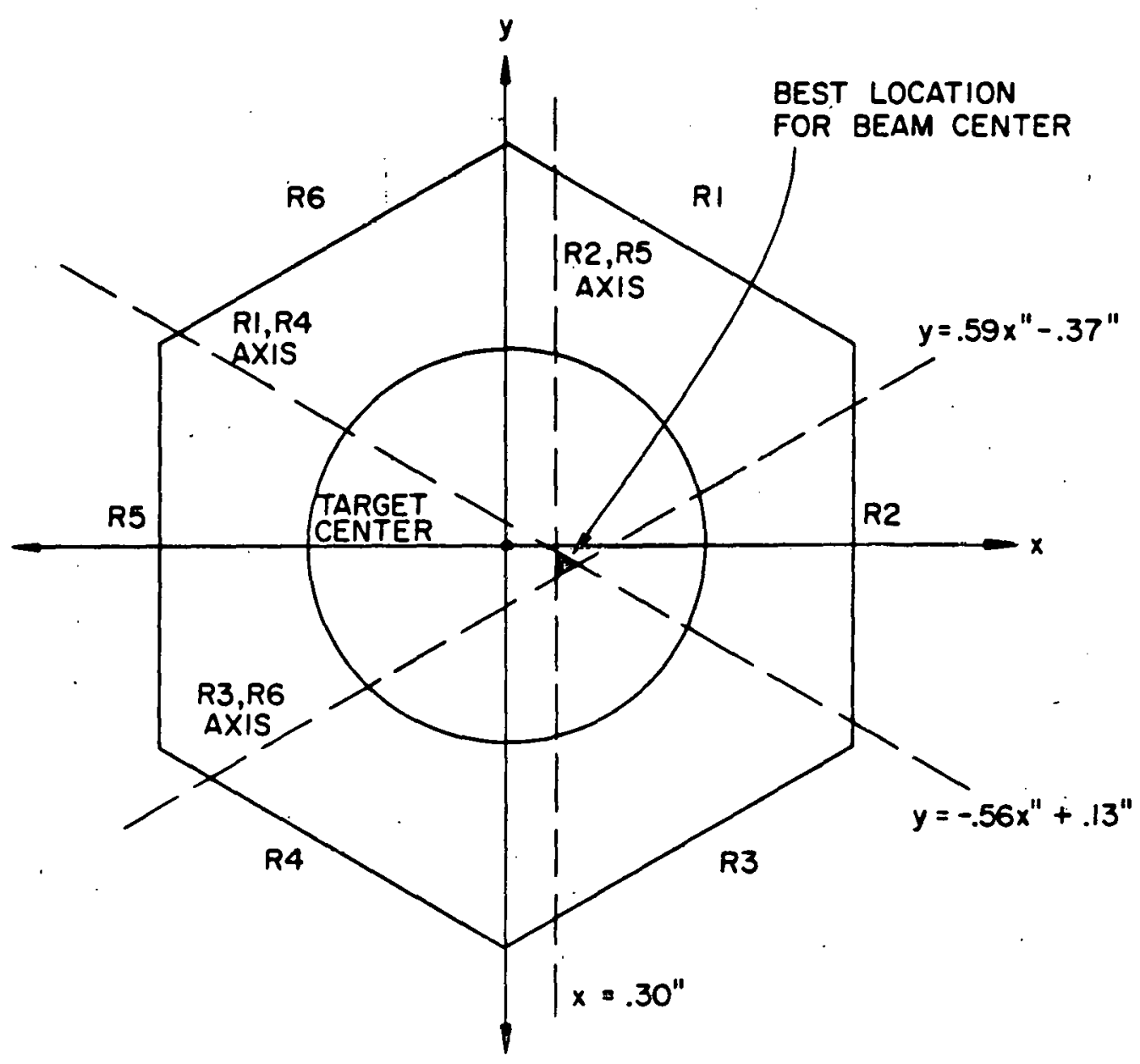

Figure 5.4 


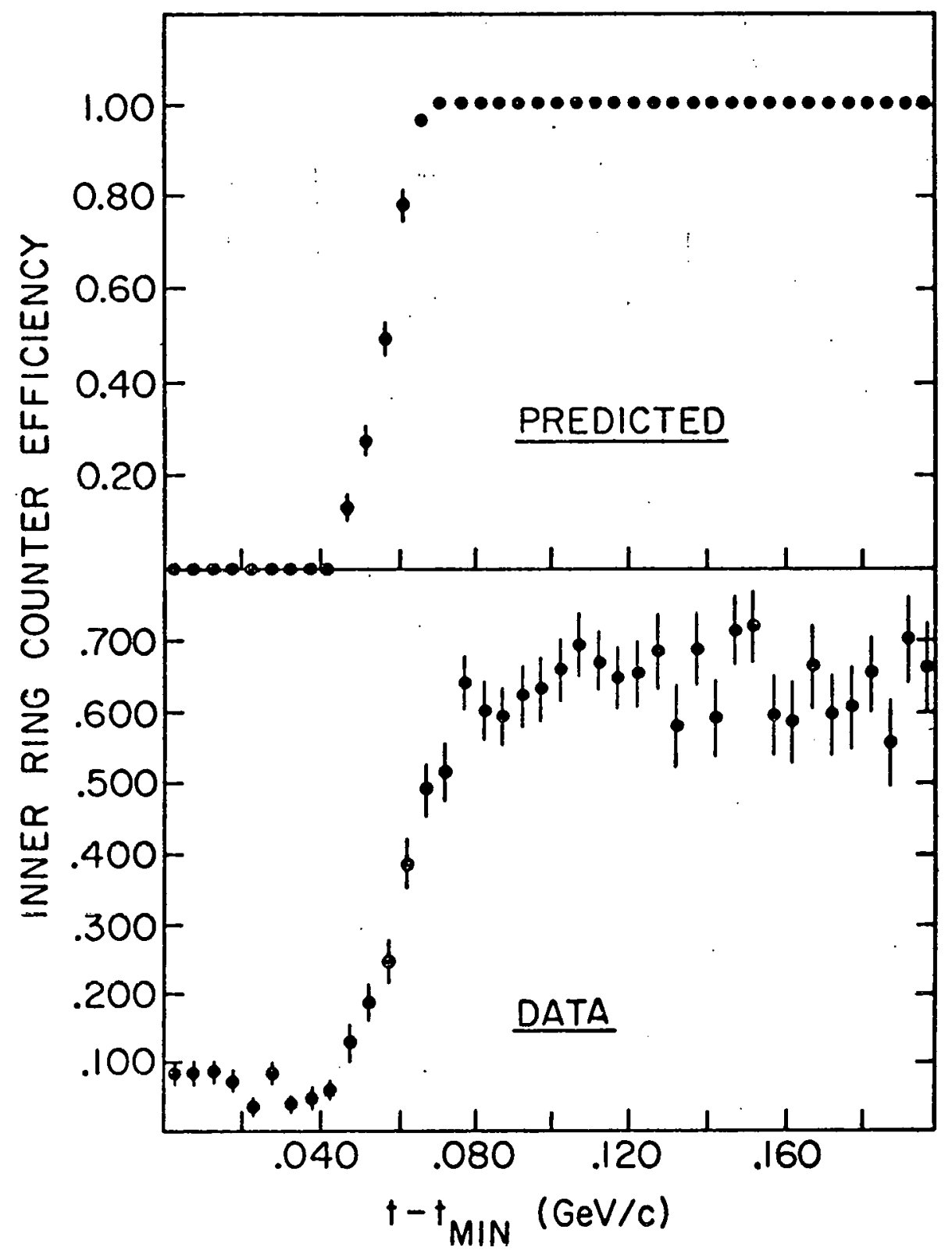

Figure 5.5 
Therefore, the counter positions, beam center, and $\mathrm{LH}_{2}$ center were determined. Coupled with the accurate range calculation for a proton, a prediction as to which scintillation counters would fire for an elastic event was made. The discriminated anode signals for each counter from an event were compared with this prediction to determine whether or not it was consistent with elastic production.

Section 2: ADC Calibration

The dynode signals from the RC were summed in groups of three and recorded by ADC's. No two adjacent counters were summed. There was no ambiguity as to which counter the dynode signal was associated with because elach anode signal was recorded individually at the coincidence register. This pulse height information was used for particle identification based on the different range-energy loss characteristics for protons and pions in this energy region.

A recoil proton with a typical energy of $t \sim .1 \mathrm{Gev}^{2} / \mathrm{c}^{2}$ $(p=330 \mathrm{MeV})$ is in the energy loss region for charged particles in matter well below the velocity for minimum ionization. Instead of a constant energy loss, we have

$$
\mathrm{dE} / \mathrm{dx} \propto 1 / \mathrm{E}
$$

so the energy loss in a scintillation counter must be computed for each event. The larger the computed energy loss, the 
larger the observed pulse height should be (assuming the phototube response is linear). Thus, the relationship

$$
C=\Delta E_{c} / P H
$$

where

$$
\begin{aligned}
& \Delta \mathrm{E}_{\mathrm{c}}=\text { Computed energy loss in } \\
& \text { scintillation counter for } \\
& \text { a recoil proton } \\
& \mathrm{PH}=\text { Observed pulse height } \\
& C=\text {. A constant, characteristic } \\
& \text { of a specific counter }
\end{aligned}
$$

is expected to hold. The number $\mathrm{C}$ will be a characteristic of the counter only for protons. A pion in the same energy range would already be minimum ionizing $(\beta \geq .96)$ and deposit ten times less energy than a proton. The pulse height would be a factor of ten smaller and the number $C$ ten times larger.'

To measure $C$ for each counter, a sample of 2-track events with a 2-pion mass within $+/-150 \mathrm{MeV}$ of the $\rho$ mass was used. On the assumption that all tracks observed in the RC were protons, plots of $C$ for each counter were made. Figure 5.6 shows the calibration constant C (called "calb." in graph) for two typical recoil counters compared with the raw ADC distribution observed for those counters. There is a clear, 


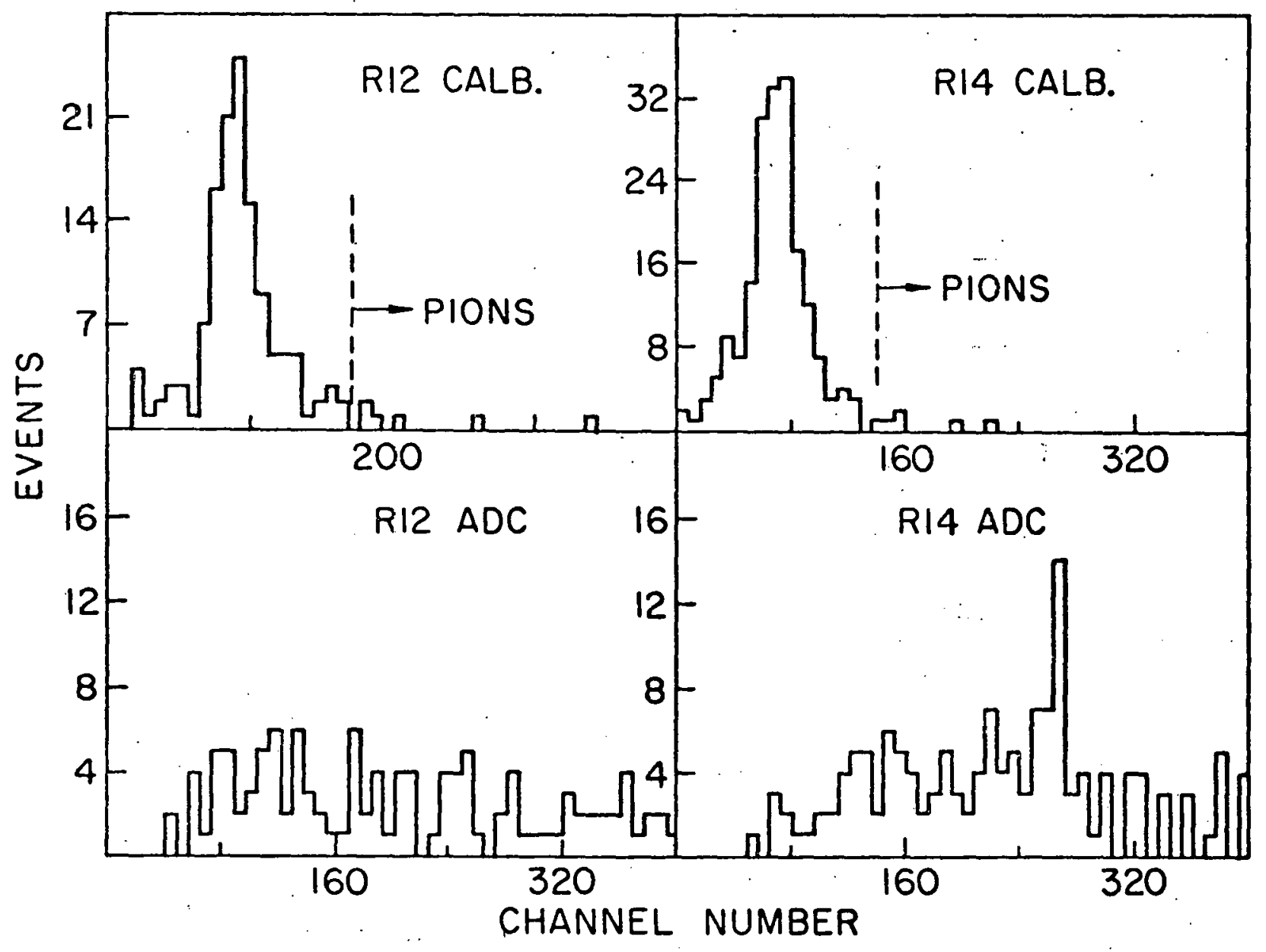

Figure 5.6 
well identified peak for each counter. This peak corresponds to protons. Thus, by computing expected proton energy losses and studying the pulse heights observed in the $R C$, proton identification is attained.

Section 3: Resolution

When RC6 predicts which RC counter should be "on"'for' an elastic event, it makes allowances for multiple scattering and range straggling. It was found that these two effects alone were not enough to account for the broadening actually observed in position space (figure 5.2) or in momentum space (figure 5.5). This implied that the downstream spectrometer resolution was affecting the ability of $\mathrm{RC} 6$ to predict elastic events because either the vertex, $z$, and/or $t$ for the event was poorly measured.

Analytical expressions were derived to represent the expected measurement errors in $z$ and $t$ on an event-by-event basis. The many correlations between measurement errors make a detailed solution to this problem impossible. A qualitative solution was obtained by retaining only the most necessary correlations between uncertainties. The measurement errors are due predominantly to the uncertainty in the bend angle for a track, which becomes extremely small for high energy tracks, and to the finite wire spacing of the MWPC system. Another bend-angle error is introduced when projecting tracks upstream of $P \emptyset$ and through $M I$ to an intersection point in the target. 
Rather than burden the reader with any of the details, I will simply quote the results. For the vertex resolution, we have:

$$
\sigma_{z}=\frac{\sqrt{2} \cdot Z}{\left|\hat{N}_{1}-\hat{N}_{2}\right|} \cdot \Delta \hat{N} .
$$

where

$$
\begin{aligned}
\sigma_{z}= & \text { standard deviation for the vertex resolution } \\
z & =\text { z position of the } 2 \text {-track intersection } \\
\hat{N}_{i}= & \text { direction cosine of track i at the vertex } \\
\Delta \hat{\mathrm{N}}= & \text {.l milliradians - the angular } \\
& \begin{array}{l}
\text { resolution of the MWP system } \\
\text { due to the finite wire spacing }
\end{array}
\end{aligned}
$$

The dominant effect is the inverse dependence on the opening angle. Two tracks with a small opening angle would be expected to have a large uncertainty in their point of closest approach. For the $t$ resolution we have:

$$
\begin{aligned}
& \sigma_{t} \approx 2 \sqrt{t}\left[\delta P_{x}^{2} \cdot \sin ^{2} \phi+\delta P_{y}^{2} \cdot \cos ^{2} \phi\right]^{1 / 2} \\
& \delta P_{x} \approx\left[\rho+\frac{\sqrt{10}}{P_{1}^{M 2}}\left(P_{1}^{2} \cdot \hat{N}_{x_{1}}+P_{2}^{2} \cdot \hat{N}_{x_{2}}\right)\right] \cdot \Delta \hat{N} \\
& \delta P_{y}=\left[P \cdot \sqrt{1+10 R_{\perp}^{2}}+\frac{\sqrt{10}}{P_{1}^{M 2}}\left(P_{1}^{2} \cdot \hat{N}_{y_{1}}+P_{2}^{2} \cdot \hat{N}_{y_{2}}\right)\right] \cdot \Delta \hat{N}
\end{aligned}
$$


where

$$
\begin{aligned}
& \sigma_{t}=\text { standard deviation for the } t \text { resolution } \\
& \phi \quad=\text { azimuthal angle of the 2-track state } \\
& \mathrm{P}=\text { total 2-track momentum } \\
& \mathrm{P}_{\mathrm{i}} \quad=\text { momentum of track } i \\
& \mathrm{P}_{\perp}^{M 2}=\underset{\text { magnet, } M 2}{\text { field strength of the main analyzing }} \\
& \begin{aligned}
\mathrm{R}_{\perp}= & \text { The ratio of the field strengths of the } \\
& \text { two magnets, MI/M2 }
\end{aligned} \\
& \hat{\mathrm{N}}_{\mathrm{x}_{i}}=\underset{\text { target }}{\mathrm{x} \text { direction cosine of track } i \text { at the }} \\
& \hat{\mathrm{N}}_{y_{i}}=\underset{\text { target }}{y} \text { direction cosine of track } i \text { at the } \\
& \Delta \hat{\mathrm{N}}=.1 \text { milliradians }
\end{aligned}
$$

The dominant features in equation 5.3 are that $\sigma_{t}$ increases like the $\sqrt{t}$ and that $\delta \mathrm{P}_{x}$ and $\delta \mathrm{P}_{y}$ are asymmetric due to the direction of the magnetic field. The factor $\sqrt{10}$ comes about because the angular resolution for a line segment in the two wire chambers downstream of M2 (P3\& P4, figure 2.3) is three times larger than the angular resolution for a line segment in the three upstream chambers (Pø, PI\& P2). Note that the resolution improves it we increase M2's field strength, $P_{\perp}^{M 2}$, thus obtaining larger bend angles. The resolution degrades with increasing momentum. The resolution is worse in the bend plane, $P_{y}$, than in the non-bend plane, $\mathrm{P}_{\mathbf{X}}$ 
To test whether these formulas were capable of estimating measurement errors on an event-by-event basis, a detailed detector simulation program was used (chapter 6). The simulated "measured" quantities were used, and plots of $\left(z_{\text {GENERATEO }}-z_{\text {MEASURED }}\right)$ and $\left(t_{\text {GENERATED }}-t_{\text {MEASURED }}\right)$ were made. The widths of these distributions in different regions of opening angle and $t_{r}$ respectively, were taken as estimates of the true resolution, $\sigma_{t}$ and $\sigma_{z}$, for the detector (figure 5.7). Equations 5.2 and 5.3 were then used to compute $\sigma_{z}$ and $\sigma_{t}$ analytically for each event from a sample of $\rho$ data. These results are overplotted with the Monte Carlo results in figure 5.7. The agreement is excellent.

RC6 uses equations 5.2 and 5.3 to compute an estimated uncertainty in the vertex position and $t$ for each event. RC6 makes no statements about the elasticity of an event stronger than what is allowed by the resolution for the event. The uncertainty in $t$ induces an uncertainty in the direction and the most probable range for a recoil proton. The uncertainty in $z$ implies an uncertainty in whether or not a recoil proton entered the fiducial volume of the recoil counter (figure 2.5B). 

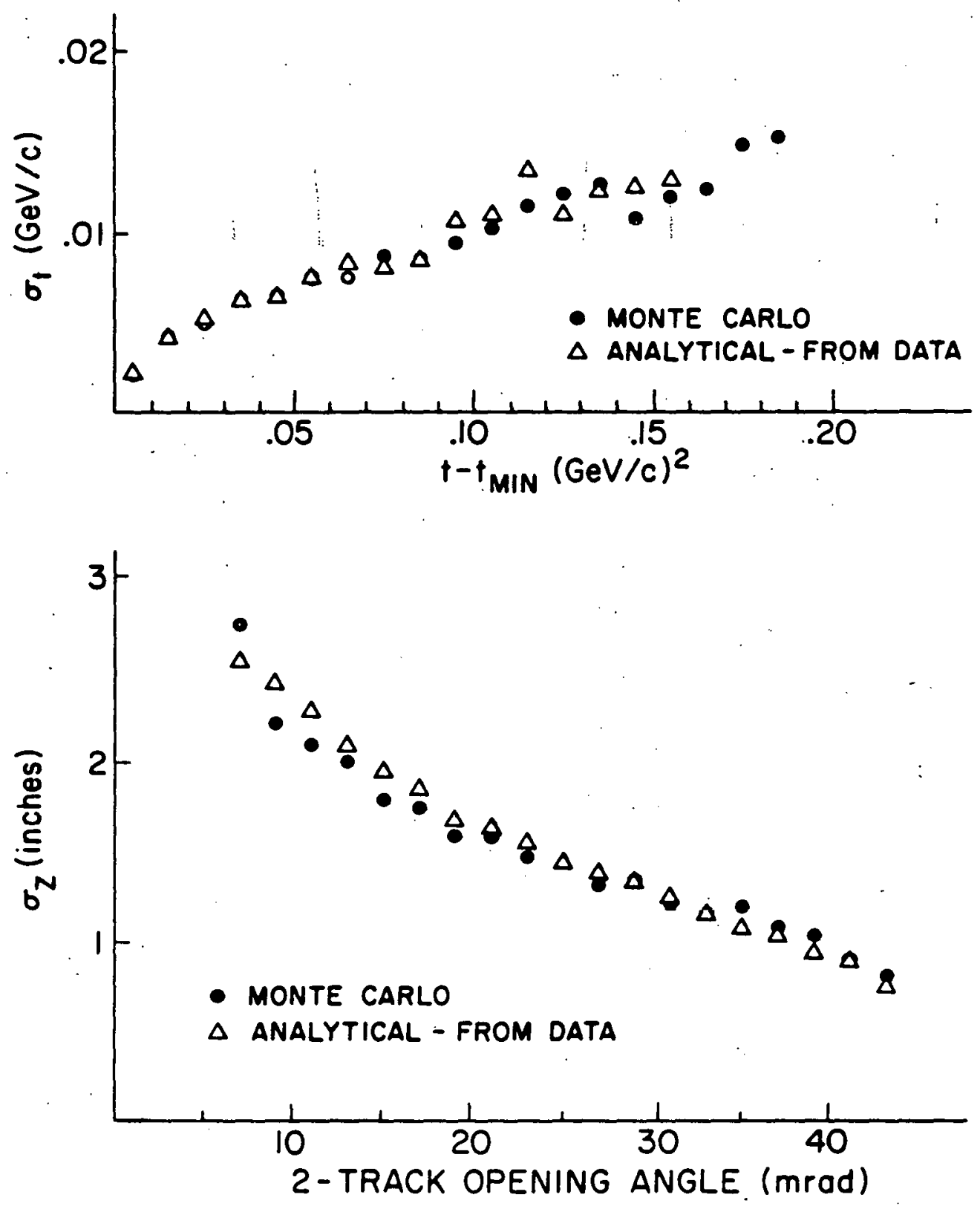

Figure 5.7 
The typical magnitude of these uncertainties for the $\rho$ is:

$$
\begin{gathered}
\sigma_{z}=+/-2 " \\
\sigma_{t}(t=.05)=+/-.007 \mathrm{GeV}^{2} / \mathrm{c}^{2}
\end{gathered}
$$

At $t=.05 \mathrm{GeV}^{2} / \mathrm{c}^{2}$ a proton is near threshold for exiting the $\mathrm{LH}_{2}$ target. At this $t_{1}$ a proton has a probable range of approximately $3.5^{\prime \prime}$ in $\mathrm{LH}_{2} \cdot \mathrm{A} 158$ uncertainty in $t$ is therefore consistent with the proton actually emerging from the target and triggering the recoil detector. RC6 allows for this and other types of problems arising from resolution effects. The induced uncertainty in the projected position due to the incertainty in $t$ is typically $10 \%$.

Listed below are all of the effects taken into account in determining acceptance as an elastic event:

1) predicted range of proton through material in target

2) straggling tolerance about this range

3) multiple scattering radius due to target and detector materials

4) correction for net beam divergence

5) error in range and direction due to measurement errors in $t$

6) uncertainty in $z$ of the target vertex in in deciding whether an event is in RC fiducial volume

7) other geometrical factors (cracks, etc.)

8) calculation of proton energy loss in scintillators and comparison of ADCS with calibration constants

9) accidental hit rates - whether the number of unassociated hits is consistent with expected backround for this event type 
Appendix $D$ details how this information is used to make the categories corresponding to elastic and inelastic events.

Section 4: Recoil Analysis of the $k_{L}^{0}$ Data

Recall that one of the major backgrounds to elastic $\phi$ photoproduction is $\phi$ production from $k_{L}^{0}$ mesons:

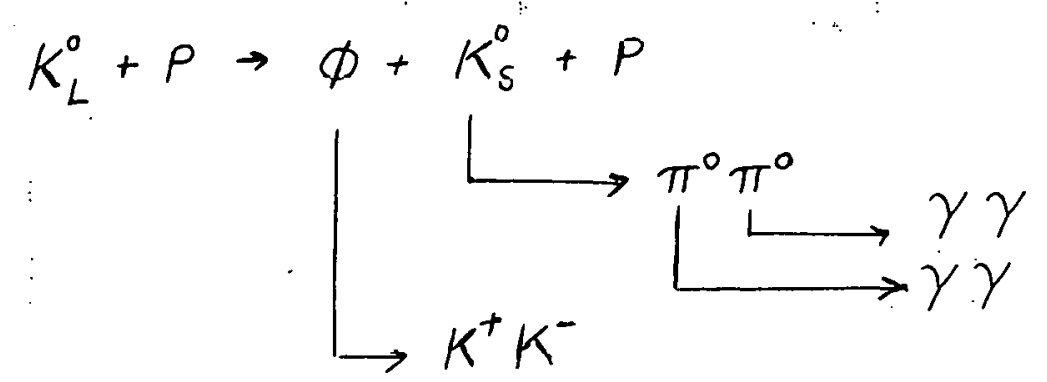

Using the special runs taken with 6 radiation lengths of lead in the beam to attenuate the photon content, the recoil analysis RC6 was performed on the $K_{L}^{0}$ production data. All of the diffractive event cuts were applied to the $K_{L}^{0}$ data that were applied to the photoproduced data (chapter 3). Also, it was required that there be at least one kaon identified by the Cerenkov counter system. This Cerenkov requirement enhanced the signal to noise ratio. Figure 5.8 shows the beautiful result that essentially all of the $\phi$ signal from $K_{L}^{0}$ production is classified as inelastic by the analysis of the recoil detector. 


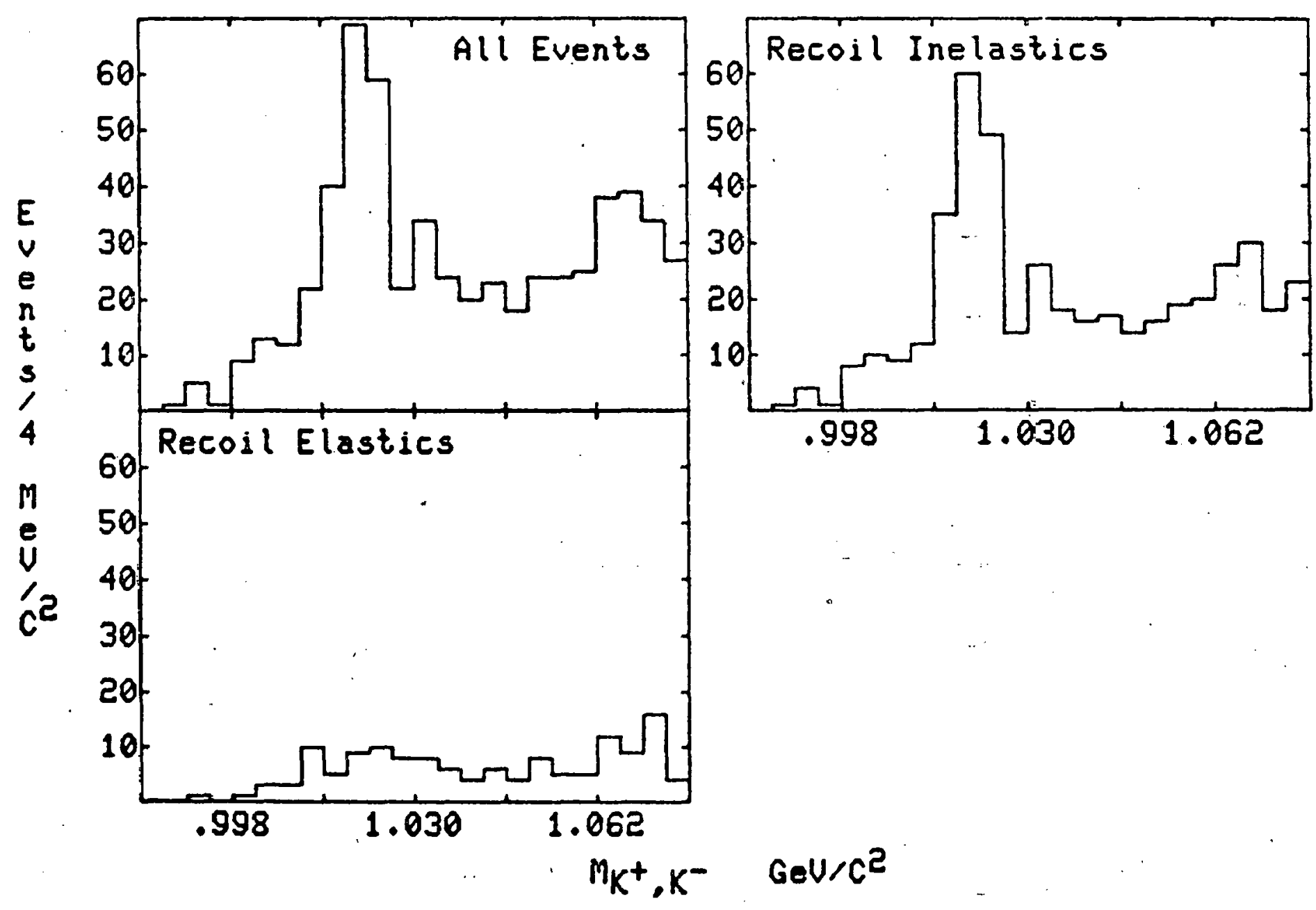

Figure 5.8 


\section{Chapter VI}

The Monte Carlo

A detector simulation program was written to model the geometry, device performance, and hardware logic of the actual detector as described in chapter 2 . The detection efficiency and resolution for measuring the final states considered in this thesis were determined by analyzing the Monte carlo.

Events were generated uniformly over the target volume with an energy distribution like that of the observed photon spectrum (figure 2.3). The parent state was allowed to decay with a $\sin ^{2} \theta_{C M}$ decay angle distribution. This is the decay angle distribution expected with s-channel helicity conservation. S-channel helicity conservation is consistent with the decay angle distribution observed in the datá [21].

The mass distribution for the $\rho$ was modeled after the mass distribution described by Spital and Yennie [2] for a $\rho$ resonance interfering with a non-resonant 2-pion background. Figure 6.1A shows the generated ai-pion invariant mass spectrum from the $\rho$ Monte carlo. The $\ell$ mass was generated between $285 \mathrm{MeV}$ and $1085 \mathrm{MeV}$. This broad 2-pion mass spectrum was generated because of the variation in the acceptance as a function of mass. Figure 6.1B shows the acceptance as a function of $2 \pi$ mass, integrated over all energies, for our detector. As illustrated, to use this parametrization of the $2 \pi$ spectrum, to fit the data, a correction for the acceptance as a function of mass must be 


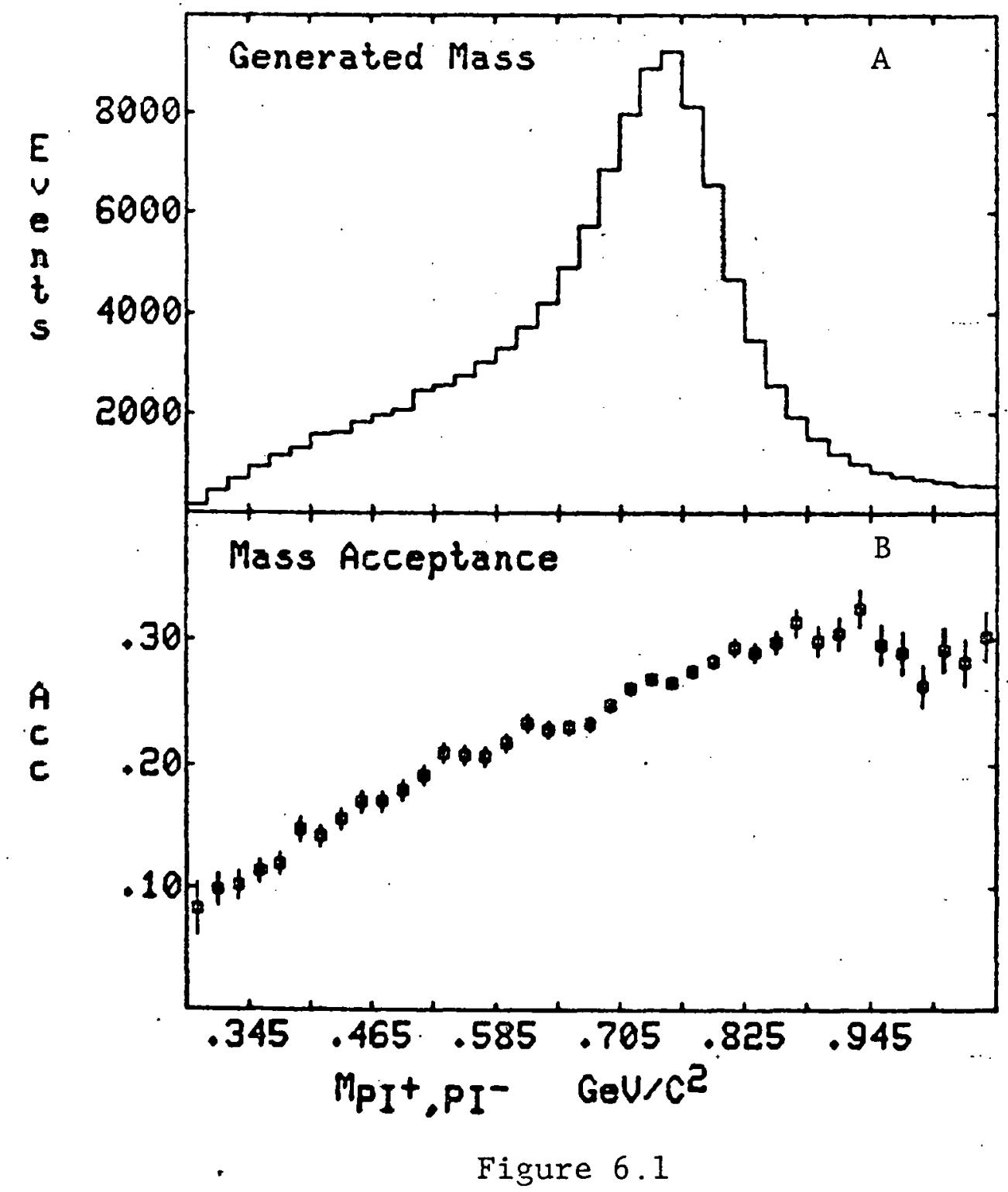


made. Generating this mass distribution allows the study of the reflected low mass di-pion background under the $\varnothing$ and reproduces observed target distributions and opening angle distributions as well.

The $\phi$ mass was generated as a Breit-Wigner resonance with a mean of $1019.6 \mathrm{MeV}$ and a width of $4.1 \mathrm{MeV}$.

The $t$ distribution was generated as $\exp (-b t)$ with $b=8.5$ (6.5) $(\mathrm{GeV} / \mathrm{c})^{-2}$. for the $\rho(\phi)$. The acceptance is very insensitive to the t distribution, varying by only 208 if a slope of $b=60(\mathrm{GeV} / \mathrm{c})^{-2}$ is used. The values used here are in fact very close to the measured values (chapters 7 and 8 ).

Once $t$ is determined, the recoil proton simulation is performed. The proton loses energy in the target materials, undergoes range straggling, and multiple scatters in a fashion similar to what is described in chapter 5. Counter inefficiencies and accidental hits are also included. ADC pulses are generated using the calibration constants discussed in chapter 5, section 3. Evidence of the accuracy of the recoil detector Monte Carlo is shown in figure 6.2. The pounts with the error bars are positively identified elastic events (see appendix D) from a sample of $\rho$ data. 
The bar graphs are the predicted $t$ distributions for events of this type for three different assumptions by the Monte Carlo:

1) The inner ring scintillation counter is triggered in the air before the proton reaches the inner ring.

2) The inner ring counter is triggered when the proton reaches the front edge of the scintillator.

3) The inner ring counter is triggered only atter the. proton reaches the outside edge of the scintiliator.

The t-distribution predicted by the Monte Carlo is quite sensitive to this test in the threshold region. Figure 6.2 shows clearly how well assumption \#2, the most realistic, agrees with the data.

The Monte Carlo then boosts the four vectors for the daughter particles to the laboratory frame and projects them through the apparatus, "swimming" them through the magnetic fields and setting bits for intercepted scintillation counters. If at any point a track leaves the fiducial volume of the dector the projection 1 s terminaled.

The performance of the MWPC's was modeled by truncating the true position of a track in the chamber to one on a lattice with a granularity equivalent to the wire spacing in the chamber. These "measured" positions were then fit to straight line segments simulating a measured track. By intersecting these "measured" line segments in the bend plane of the ma1n analyzing magnet, M2, a "measured" momentum was obtained from the bend angle. This technique works extremely 


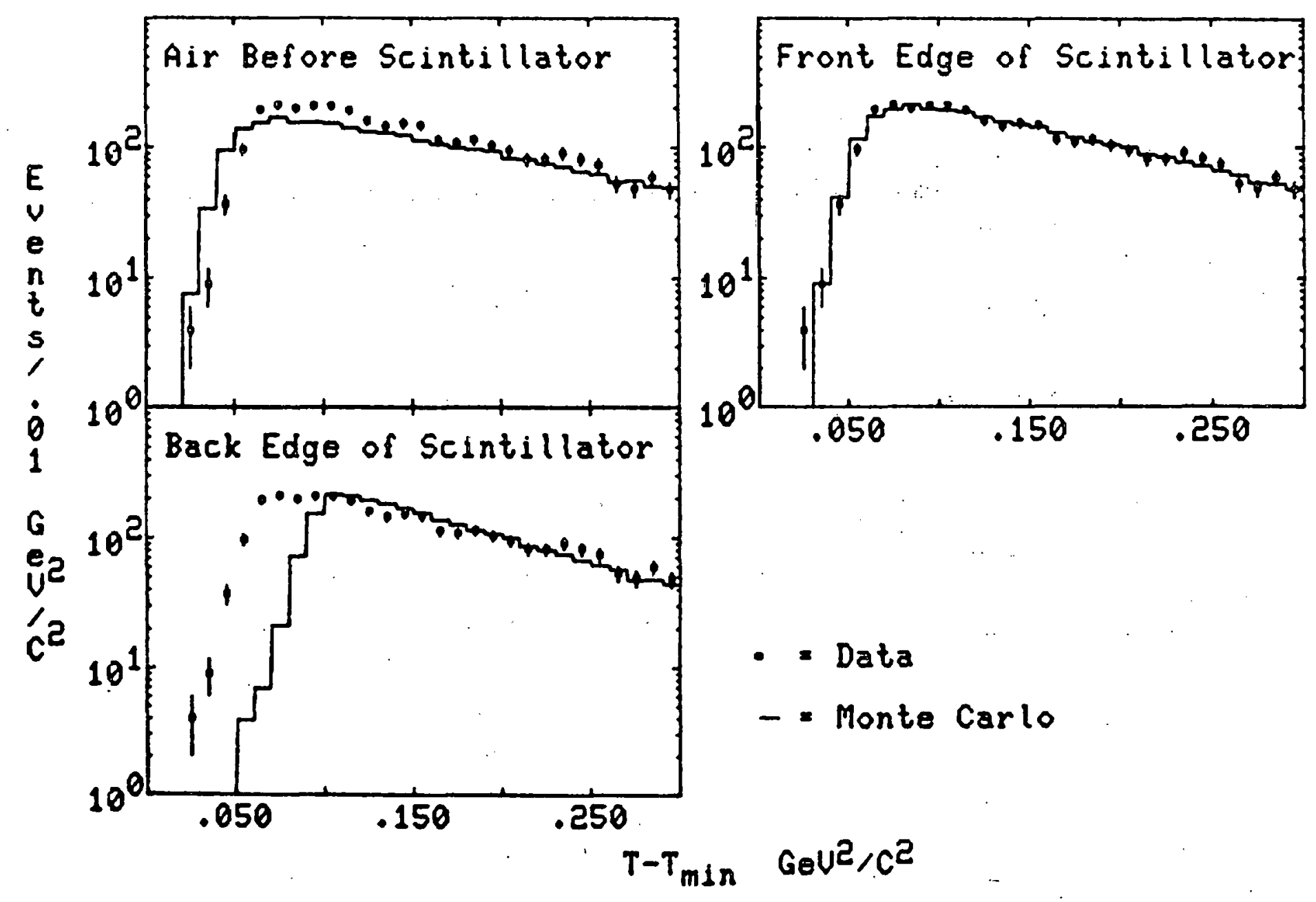

Figure 6.2 
well. for modeling the resolution as can be seen by comparing the "measured" vertex distribution at the target with that of the real data (figure 6.3). It also faithfully reproduces the observed width of the $\psi(3095)$ [8]. The slight excess of events at each end of the target distribution for the data are due to coherent $\rho$ production from the mylar endcaps of the $\mathrm{LH}_{2}$ target vessel. This mechanism is not included, in, the Monte Carlo, and is subtracted in the analysis (see chapter 8).

If a charged track went through either Cerenkov counter, it was allowed to radiate photons randomly along the cerenkov cone. These photons were then traced to phototubes where a pulse height was set according to the calibration and resolution parameters relevant to that phototube.

Charged tracks striking the lead glass array: lost a fraction of their energy according to the following scheme:

Fraction of Generated Events Fractional Energy Loss, f

$\pi \quad \pi \quad K$

$\underline{E<30} \quad \underline{3 \theta<E<50} \quad \underline{E} \geq \underline{50} \quad \underline{A} 1 \mathrm{E} \quad \underline{E=E}$ LOSS $\angle E$ TRACK

\begin{tabular}{|c|c|c|c|c|}
\hline $\begin{array}{r}.30 \\
.064 \\
.35 \\
.32 \\
4.07\end{array}$ & $\begin{array}{r}.30 \\
.985 \\
.42 \\
.41 \\
5.27\end{array}$ & $\begin{array}{r}.30 \\
.19 \\
.36 \\
.40 \\
6.43\end{array}$ & $\begin{array}{r}.39 \\
.066 \\
.32 \\
.29 \\
5.90\end{array}$ & $\begin{array}{l}\mathrm{f}=\emptyset \\
\emptyset<\mathrm{f}<. \emptyset 5 \\
. \emptyset 5<\mathrm{f}<\mathrm{fl} \\
\mathrm{fl} \\
\mathrm{b}: \mathrm{dN} / \mathrm{df}\end{array}$ \\
\hline
\end{tabular}




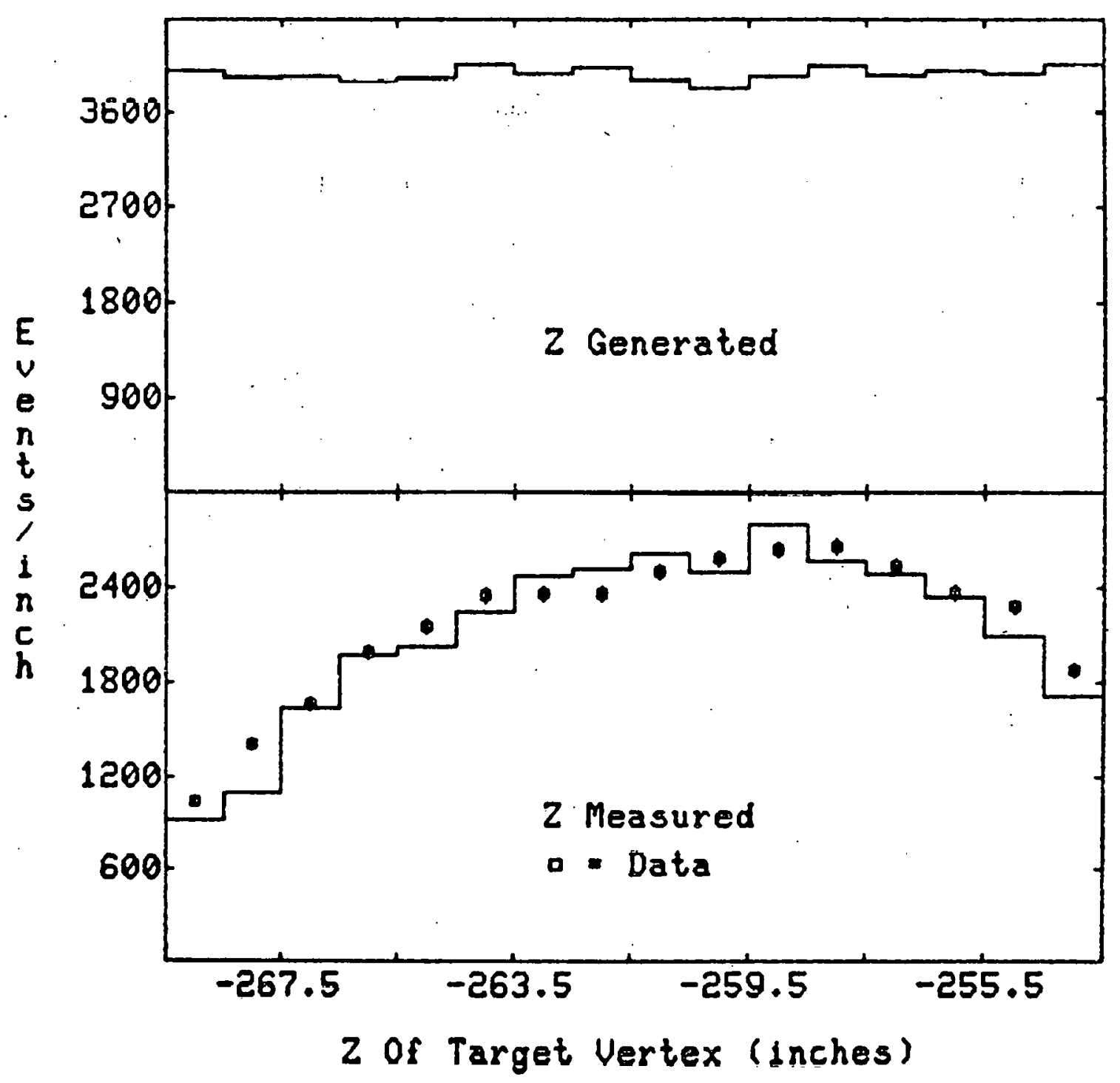

Figure 6.3 
The parametrization described above for generating charged track energy loss in the lead glass array was obtained from a study of $2 \pi$ and $2 \mathrm{~K}$ events, as determined by the Cerenkov counter analysis, where at least one track was pointing at an HC module with a PAD count greater than 60 channels. The requirement that one track be associated with an HC module having a PAD count $>60$ channels obtained a sample of events, where the PAD count of the second track was relatively insensitive to trigger bias (figure 4.2 ). The unbiased track was opposite the track with an associated 60 channel HC PAD count. The frequency with which the opposite track, was associated with a lead glass shower, and the energy of the associated shower, were studied to obtain the above model. .

As discussed in chapter 4 , there was no need to model the behavior of the hadron calorimeter for this study.

The results of the Monte Carlo were written out to magnetic tape in a format consistent with the format of a reconstructed data tape. This facilitated analyzing the Monte Carlo tapes with the identical programs used for the data analysis and thereby unfolded any systematic bias or errors in these programs. 
103

Chapter VII

$\rho$ Analysis

Elastic $\rho$ photoproduction, $\gamma+p \rightarrow \rho\left(\rightarrow \pi^{+} \pi^{-}\right)+p$ has been observed for incident photon energies from 35 to $225 \mathrm{GeV}$. The four momentum transfer squared $(t)$ distributions have been measured from $\emptyset$ to $1 \mathrm{Gev}^{2} / \mathrm{c}^{2}$ in four different energy regions. The fraction of diffractive $\rho$ :production that is inelastic has been determined as a function of $t$. A total of 42,000 photoproduced 2-track events with a di-pion mass from 285 to $1085 \mathrm{MeV}$ were analyzed.

The $\rho$ is a broad resonance with a mass shape that is skewed towards lower mass when compared to a relativistic p-wave Breit-wigner shape. To parametrize the $2 \pi$ mass distribution, we use the boding Model [22] which successfully explains the skewing as an interference effect between the $\rho$ and a non-resonant $\pi^{+} \pi^{-}$background. In fitting the di-pion invariant mass distribution, we follow the suggestion of Spital and Yennie [2] and fit the spectrum to:

$$
\begin{aligned}
& \frac{d N}{d M_{\pi \pi}}=\frac{C_{0} M \cdot M_{\rho} \cdot \Gamma}{\left(M^{2}-M_{\rho}^{2}\right)^{2}+M_{\rho}^{2} \Gamma^{2}}\left\{1+C_{1}\left(\frac{M_{\rho}^{2}}{M^{2}}-1\right)+C_{2}\left(\frac{M_{\rho}^{2}}{M^{2}}-1\right)^{2}\right\} \\
& (7.1)
\end{aligned}
$$


and define the $\rho$ yield as:

$$
N_{p}=\frac{\pi}{2} C_{0}
$$

$\Gamma$ is a mass dependent width [26] given by:

$$
\Gamma(M)=\left[\frac{q(M)}{q\left(M_{\rho}\right)}\right]^{3} \cdot \frac{2 \Gamma_{0}}{1+\left[q(M) / q\left(M_{\rho}\right)\right]^{2}}
$$

$q(M)$ is the magnitude of the three momentum in the $\pi^{+} \pi^{-}$ center-of-mass frame.

Figure 7.1 shows the total $2 \pi$ invariant mass spectrum in the region of the $\rho$ that satisfied all elastic event cuts (see chapter 3 ). Also shown is the residual $\mathrm{K}_{L}^{0}$ induced backround surviving these cuts.

Figure 7.2 shows a fit to the $K_{L}^{0}$ subtracted mass distribution from figure 7.1 using equation 7.1. Note the quality of the fit over the entire $80 \emptyset \mathrm{MeV}$ spectrum. The peak at $365 \mathrm{MeV}$ is a reflection of the $\phi$ meson. The best values for the mass $M_{\rho}$ and the width $\Gamma_{0}$ from this fit are:

$$
\begin{aligned}
& M_{\rho}=774+/-1 \mathrm{MeV} \\
& \Gamma_{0}=152+/-2.5 \mathrm{MeV}
\end{aligned}
$$

The mass and width of the $\rho$ were fixed at these values throughout the rest of the analysis in different energy and $t$ regions. Only the parameters $C_{0}, C_{1}$ and $C_{2}$ were allowed to vary . 


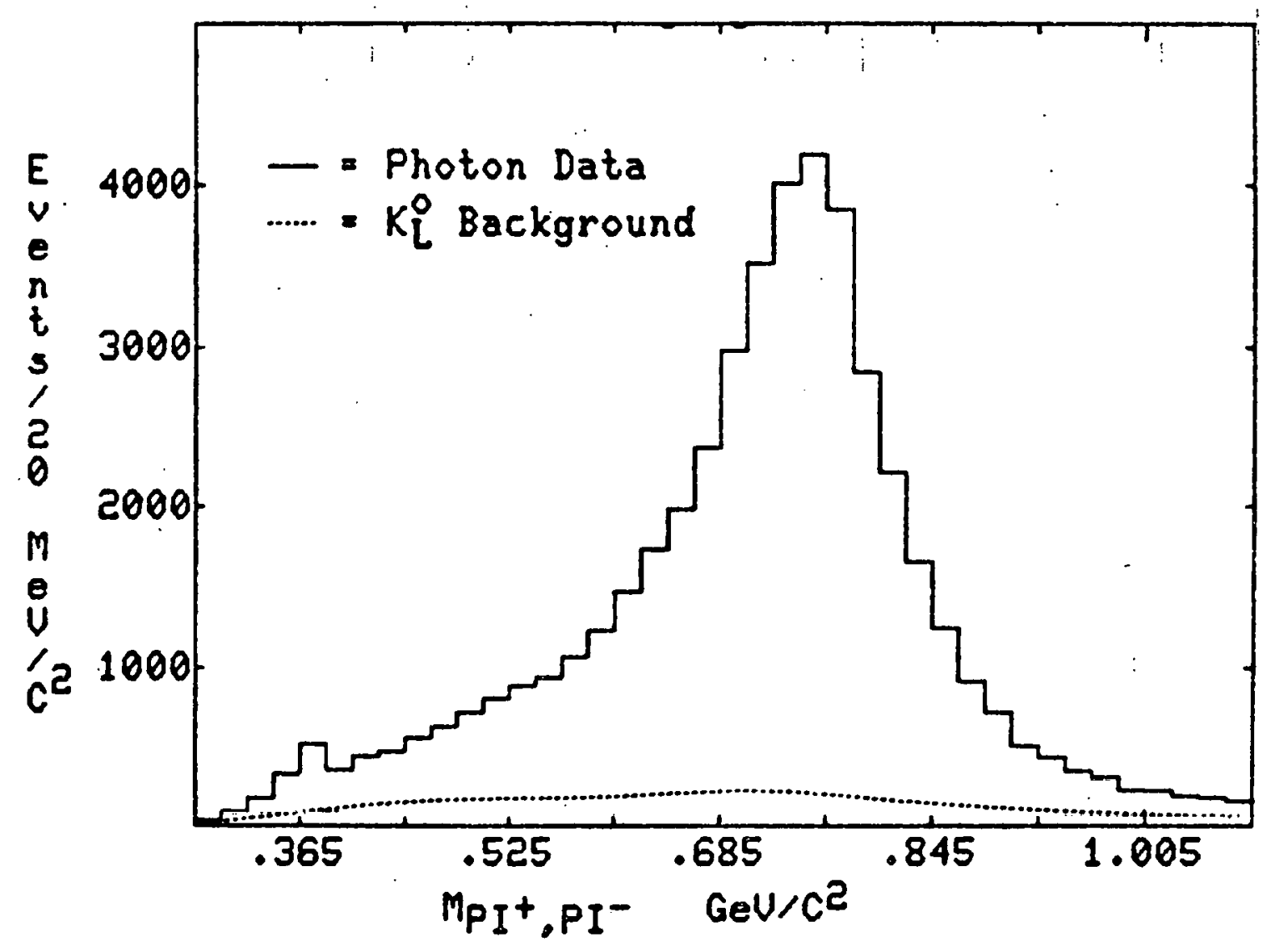

Figure 7.1 


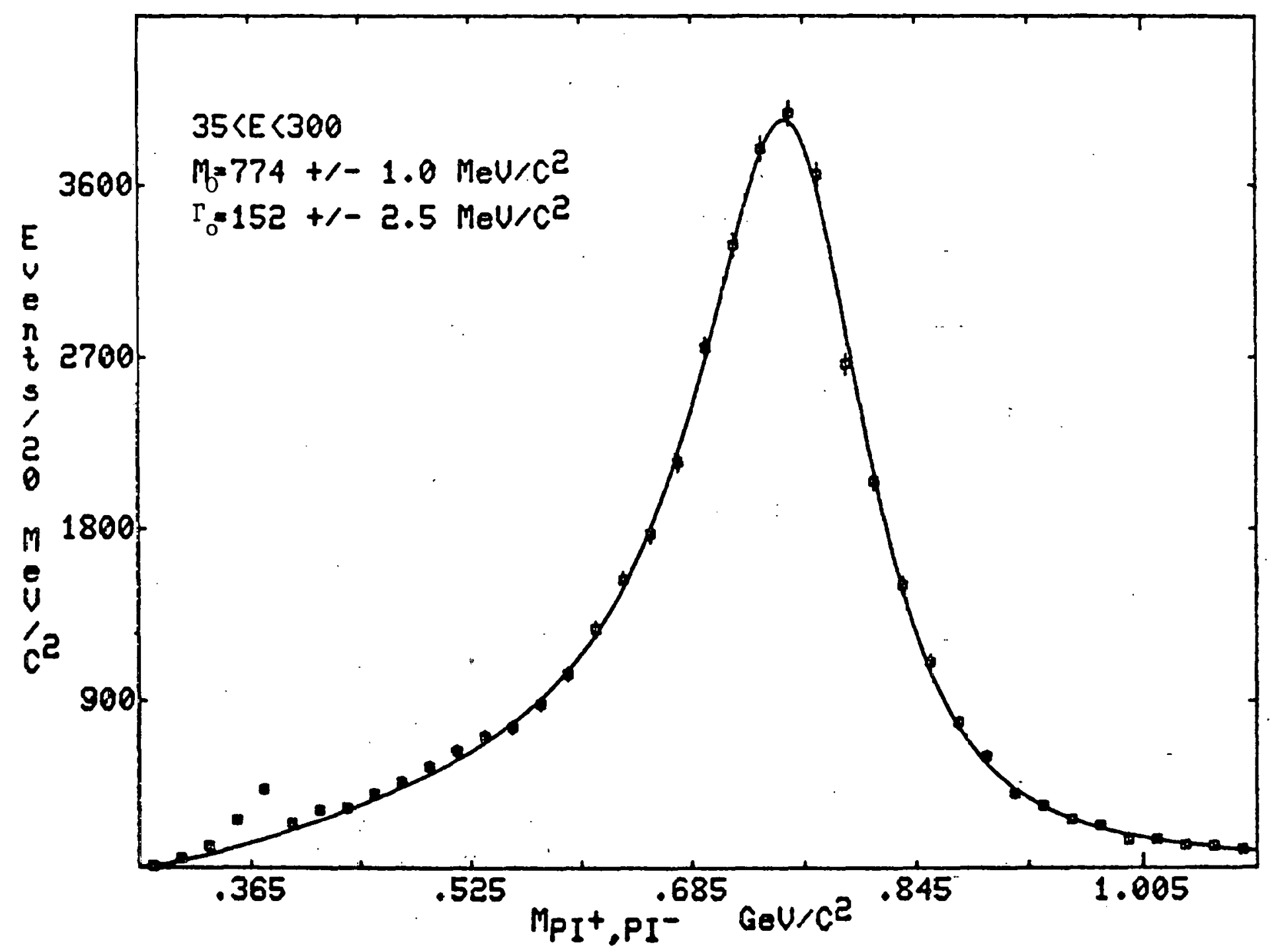

Figure 7.2 
Section 1: Calorimeter acceptance for the $p$

The technique for measuring the calorimeter acceptance has been discussed in detail in Chapter 4. The acceptance for the $e$ was found to be more clearly defined if the PAD distributions were measured in different regions of $E_{H C}$ rather than just different regions of $E_{\text {TOT }}$, where:

$$
E_{H C}=E_{T O T}-E_{L G}
$$

where

$$
\begin{aligned}
& \mathrm{E}_{H C}=\text { Energy entering calorimeter } \\
& \mathrm{E}_{\text {TOT }}=\text { Observed charged track momentum } \\
& \mathrm{E}_{L G}=\begin{array}{l}
\text { Energy associated with a charged track } \\
\text { in the lead glass array }
\end{array}
\end{aligned}
$$

The energy $\mathrm{E}_{H C}$ approximates the hadronic energy "seen" by the calorimeter more accurately than $E_{\text {TOT }}$.

The PAD distributions for the $e$ were plotted in bins of $E_{H C}$ that were $1 \emptyset \mathrm{GeV}$ wide and started at $35 \mathrm{GeV}$. The acceptance in each bin was then computed as described in chapter 4. Figure 7.3 shows the resulting plot of the calorimeter acceptance as a function of $E_{H C} \cdot$ Appendix $A$ describes how the error estinates were computed.

The Monte Carlo, as described in chapter 6, generates charged track associated showers in the lead glass array. 


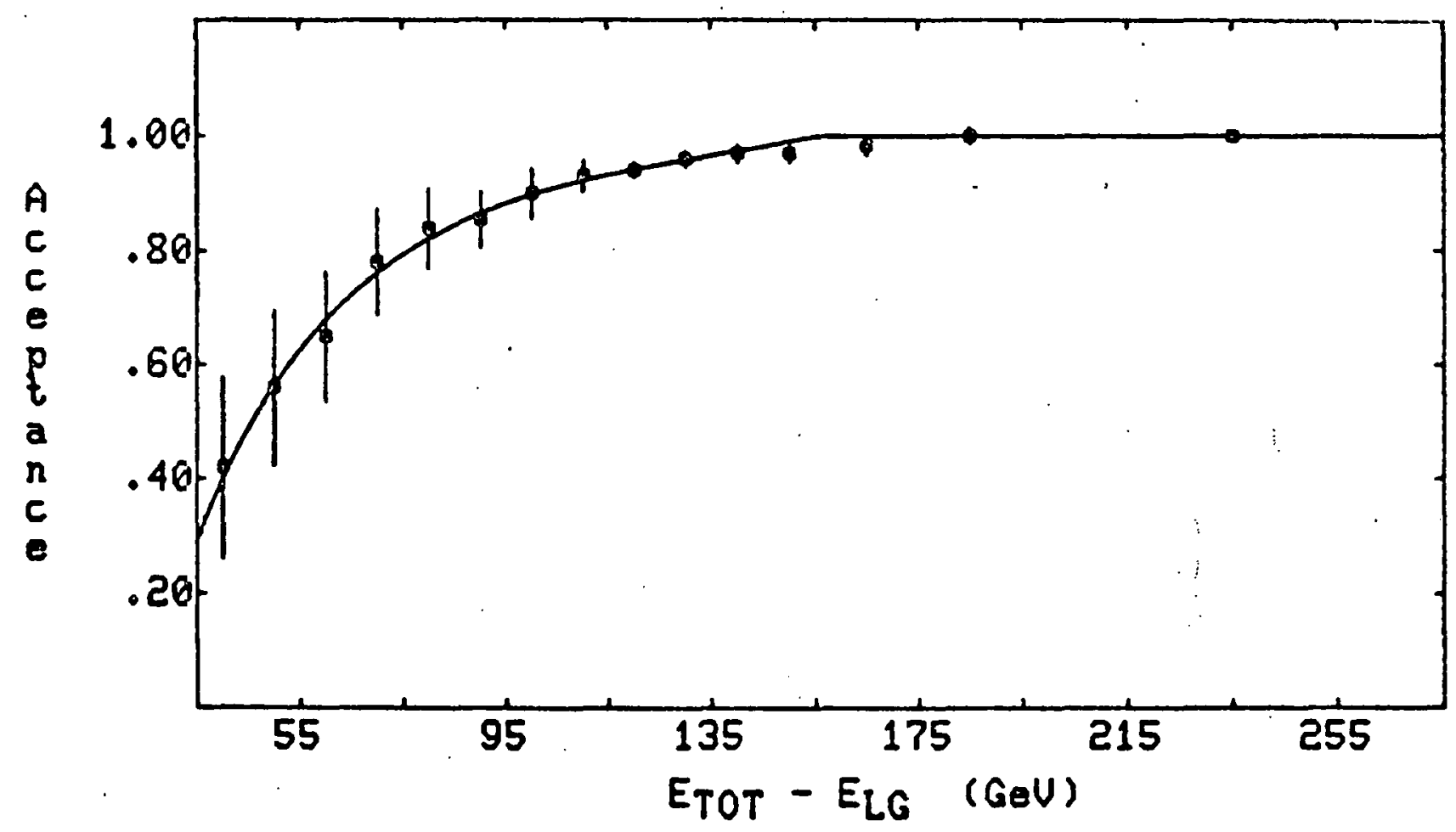

•

Figure 7.3 
The "measured" lead glass energy, $E_{L G}$, is derived from the generated shower energy by smearing it with the resolution characteristic of a lead glass block:

$$
\sigma(E) / E=.03+.05 / \sqrt{E} .
$$

Using the measured quantities, $E_{\text {ToT }}$ and $E_{L G}$, the Monte Carlo then tests $E_{H C}$ against the acceptance curve of figure 7.3, and sets the energy bussline accordingly.

Section 2: The energy dependence of the $\rho$ cross section

With the parametrization for the $\rho$ yield and the HC acceptance determined, the $\rho$ cross section can be computed in different energy regions.

The number of observed events can be expressed in terms of the cross section as:

$N=N_{\gamma} \cdot L T \cdot n t \cdot A C C \cdot \epsilon_{D E C A Y}(E) \cdot \epsilon_{M T} \cdot \prod_{i} \epsilon_{i} \cdot \sigma(\gamma+P \rightarrow e+P)$

where

$$
\begin{aligned}
N_{\gamma} & =\text { number of incident photons } \\
L T & =\text { electronic livetime } \\
n t & =\text { number of target particles/cm } \\
\epsilon_{O E C A Y}(E)= & \text { correction for pion decay in flight } \\
\epsilon_{M T}= & \text { correction for } e \text { production } \\
& \text { from target end caps } \\
A C C= & \text { acceptance (geometry and } \\
& \text { trigger) } \\
\pi \epsilon_{i}= & \text { other corrections (appendix } \mathrm{C})
\end{aligned}
$$


$\mathrm{N}$ : The number of photons on target is obtained indirectly from measurements of the quantameter charge, Q, recorded after each beam spill. The total beam power for the data analyzed here was

$$
Q=2.5909 \times 10^{-3} \quad \text { Coulombs }
$$

The quantameter was calibrated in an electron beam at SLAC and has a quantameter constant

$$
C_{Q}^{\vdots}=9800 \text { ions } / \mathrm{GeV}
$$

The flux in any energy region is obtained by using the total beam power and the shape of the photon spectrum.

$$
N_{\gamma}\left(E_{1}, E_{2}\right)=\frac{Q_{E_{1}} \int_{A_{2}}^{E_{2}} \frac{d N}{d k} d K}{C_{Q} \cdot e \cdot \int_{0}^{\infty} A_{0} \frac{d N}{d K} K d K}
$$

where

$$
\begin{aligned}
A_{0} \frac{d N}{d K}= & \begin{array}{l}
\text { number of photons with energy } \\
\text { between } k \text { and } k+\Delta k ; A_{0} \text { is } \\
\text { unknown, but divides out this } \\
\text { way }
\end{array} \\
= & \begin{array}{l}
\text { electrunic charge/ion in the } \\
\text { quantameter }
\end{array}
\end{aligned}
$$

The following representation of the photon spectrum, $\mathrm{dN} / \mathrm{dk}$ (figure 7.22), was used:

$$
\frac{d N}{d K}=\left\{\begin{array}{l}
10308 e^{-K / 6.05}+3916 e^{-k / 48.6} ; k \leq 98 \mathrm{GeV} \\
A_{1} e^{-k / 43.7} ; \quad ; \quad k \geq k<189 \mathrm{GeV} \\
A_{2} e^{-K / 23.8} ; \quad k 189 \mathrm{GeV}
\end{array}\right.
$$


111

LT: The total electronic livetime is the product of two livetimes (chapter 2 , section 7):

$L T=\left(\right.$ mastergate livetime, $\left.L T_{M G}\right) \times\left(D C\right.$ logic livetime, $\left.L T_{D C}\right)$

$$
L_{M G}=\frac{M G>1_{D T}}{M G>1_{N D T}}
$$

where MG > $1_{\text {NOT }}$ is the 2-body master gate rate and MG $>1_{\text {OT }}$ is the 2-body master gate rate with confusion logic deadtime (the confusion logic gates the DC logic)

$$
L T_{D C}=\frac{\rho_{\text {iN }} 1_{S D}}{P_{\text {iN }} 1_{S}}
$$

To obtain the DC logic livetime, the master gate was also recorded as $\mathrm{P}$ in 1 . Pin $1_{\mathrm{s}}$ is the. 2-body master gate rate at the DC logic and $P$ in $I_{S D}$ is the 2-body $P$ in 1 rate with DC logic rack deadtime. The DC logic rack deadtime is generated to allow higher level logic decisions to "settle". If the appropriate bussline conditions are met, a trigger is generated and the event is written out to tape.

$$
\begin{aligned}
& L T=L T_{M G} \times L T_{D C}=.762 \\
& n t=\frac{.0708 \mathrm{~g}}{\mathrm{~cm}^{3}} \times 40.64 \mathrm{~cm} \times \frac{1 \mathrm{MndE}}{\mathrm{g}} \times \frac{6.022 \times 10^{23}}{\mathrm{~mole}_{\mathrm{O}}} \times 10^{-30} \frac{\mathrm{cm}}{\mu b} \\
& =1.733 \times 10^{-6} \mu b^{-1} \\
& \epsilon_{D E C A \gamma}(E)=e^{-4 M X / E_{\gamma} C \tau} \text { is the probe- } \\
& \text { has decayed after traversing } \\
& \text { a distance } x \text { in the spec- } \\
& \text { trimeter (appendix } C \text {, section 2). }
\end{aligned}
$$




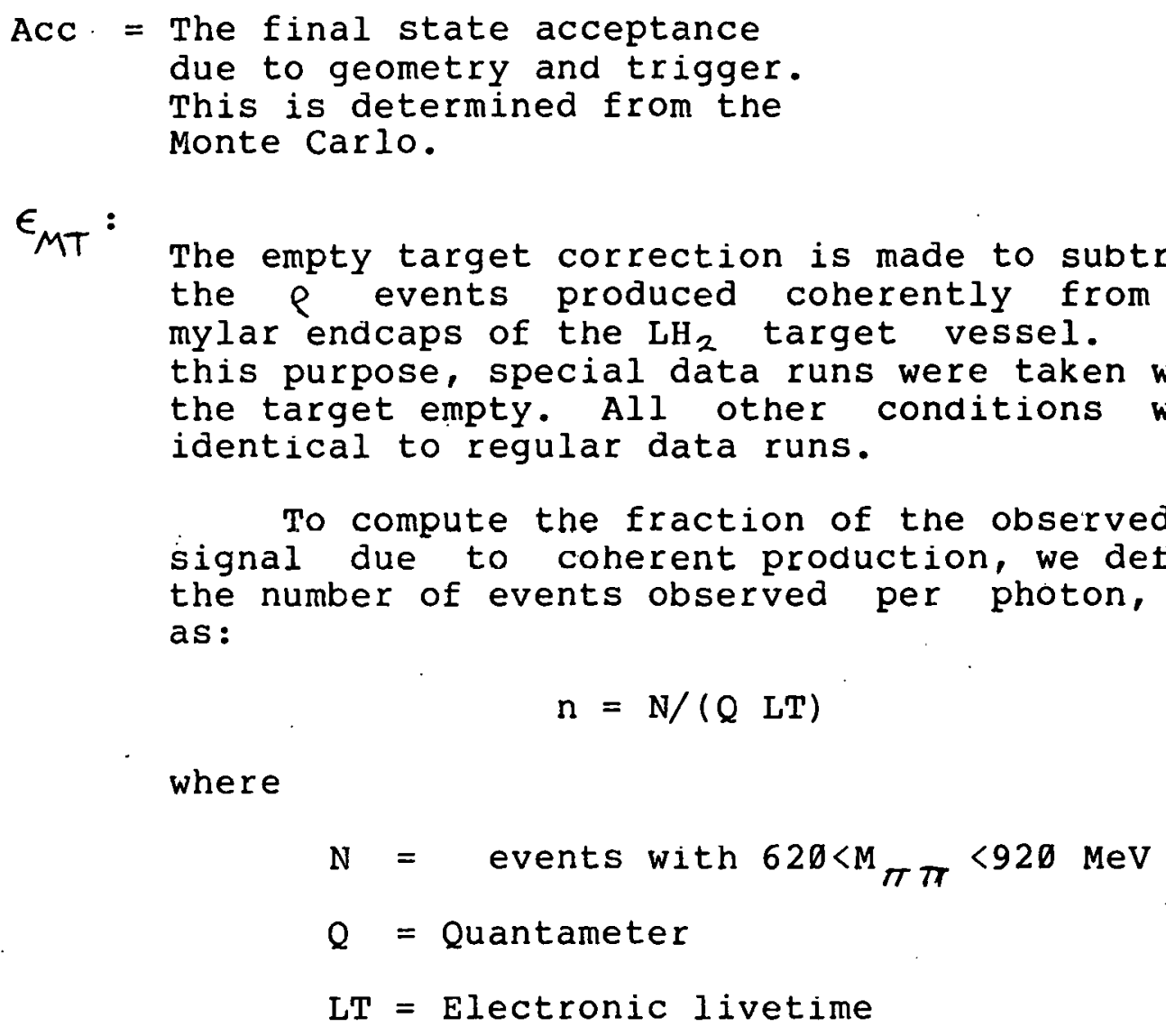

The number of events observed per photon from the empty target runs is:

$$
n_{M T}=(N /(Q \cdot L T))_{M T}
$$

where $N, Q$ and $L T$ are the appropriate quantities for the empty target runs. 
The regular data runs include both production from $\mathrm{LH}_{2}$ and from the endcaps, so the fraction due to the end caps is:

$$
\begin{aligned}
f_{M T} & =n_{M T} / n \\
& =\frac{49 \times 259.1 \times 10^{-5} \text { CoulomBs } \times .762}{43468 \times 8.264 \times 10^{-5} \text { CoulomBs } \times .821} \\
f_{M T} & =.033 \\
\epsilon_{M T} & =\frac{1}{1-f_{M T}}=1.034
\end{aligned}
$$

Putting all of this together, we obtain:

$$
\sigma(\gamma+p \rightarrow \rho+p)=\frac{9.101 \times 10^{5}}{A_{C C}(E) \cdot \epsilon_{D E C A y}(E)} \times \frac{N_{\rho}}{N_{\gamma}} \mu b
$$

The $\rho$ yield, Ne/ACC was obtained by fitting the $\mathrm{K}_{L}^{0}$-subtracted 2-pion mass spectrum in each energy region of interest. Because the acceptance varies with mass (see figure 6.13) as well as energy, the data were fit to a function $f$ where

$$
f\left(N_{\rho}, E, M_{\pi \pi}\right)=\frac{d N}{d M_{\pi \pi}}(e q .7 .1) \times \operatorname{Acc}\left(E, M_{\pi \pi}\right)
$$

and the yield taken as $(\pi / 2) c_{0}$. Recall that the mass and width for these fits were fixed at $M_{\rho}=774 \mathrm{MeV}$ and $\Gamma_{0}=152$ MeV. Figures 7.4 to 7.10 show the $K_{L}^{0}$ subtracted $2 \pi$ mass spectra and the fit $f\left(N_{\rho}, E, M_{\pi \pi}\right)$ for the 27 different energy regions analyzed. Table 7.1 summarizes the cross sections 


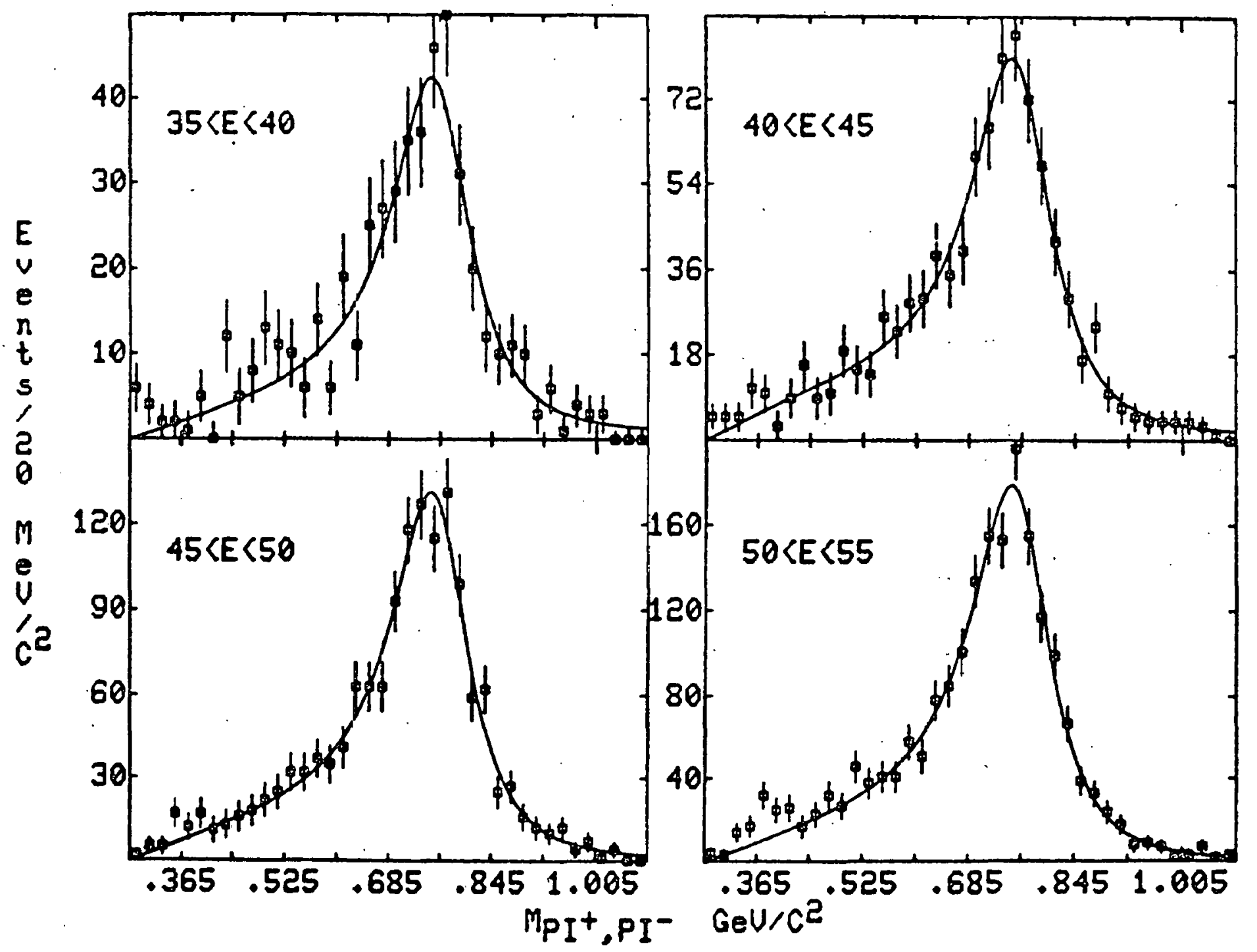

$\vec{F}$

Figure 7.4 


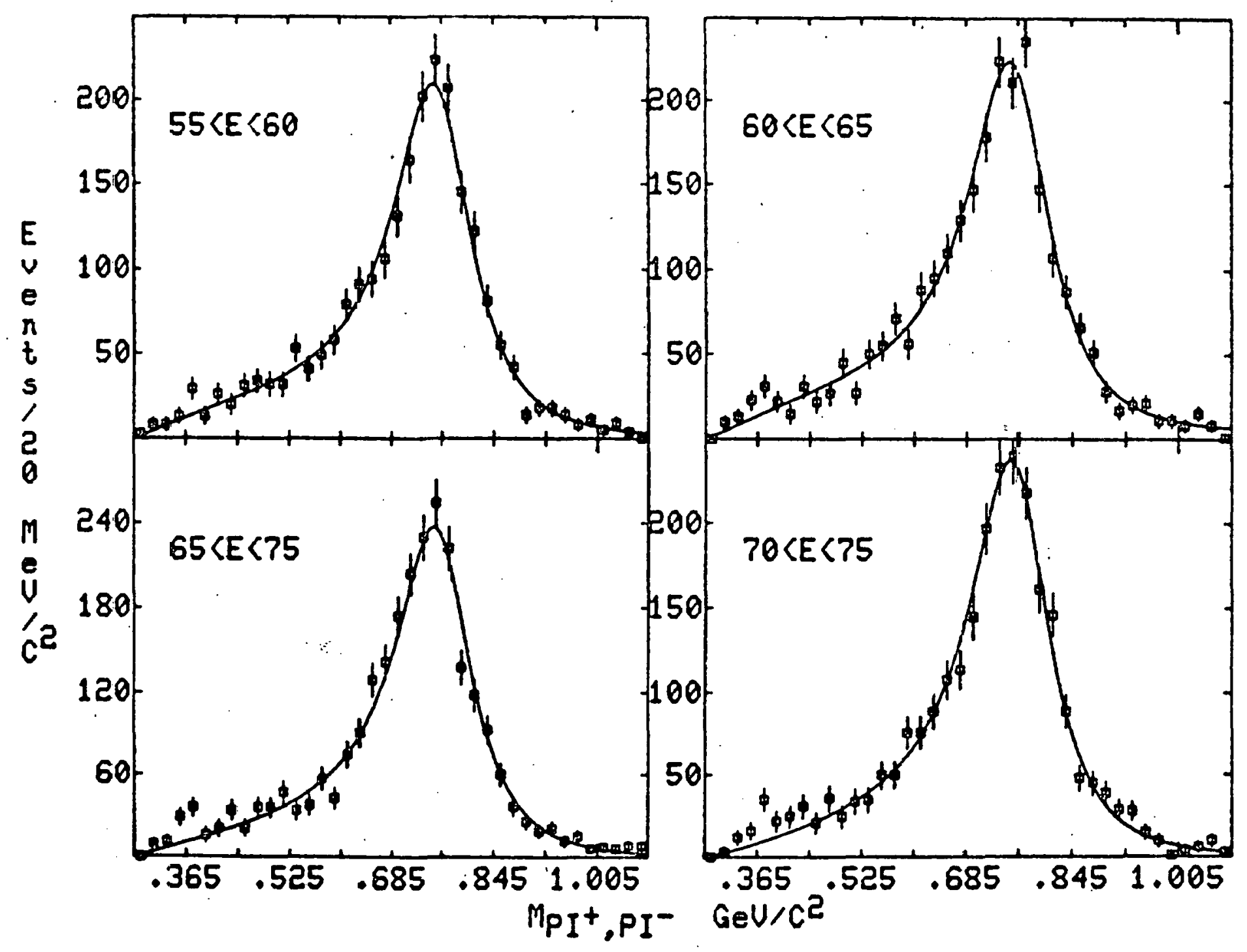

Figure 7.5 


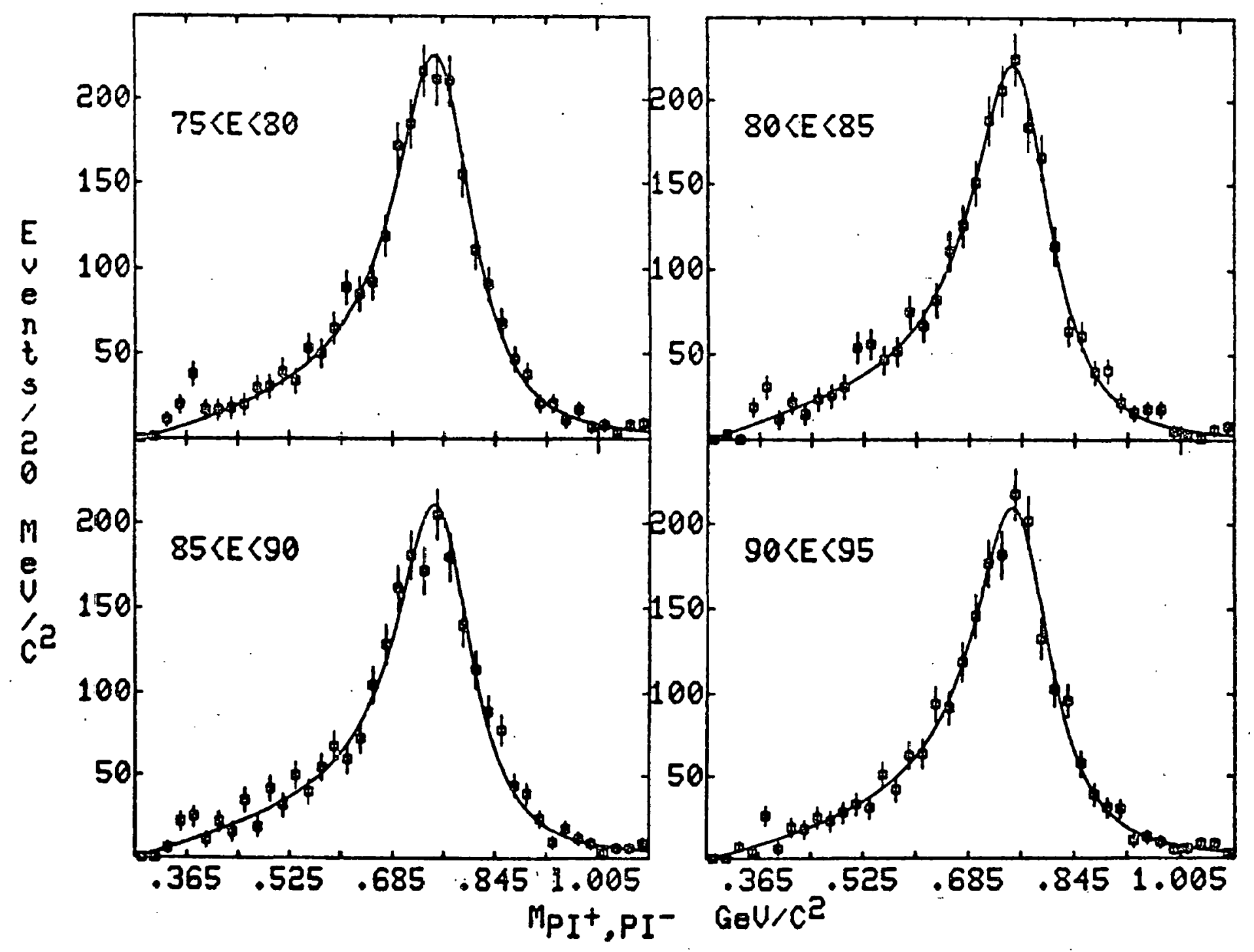

占

Figure 7.6 


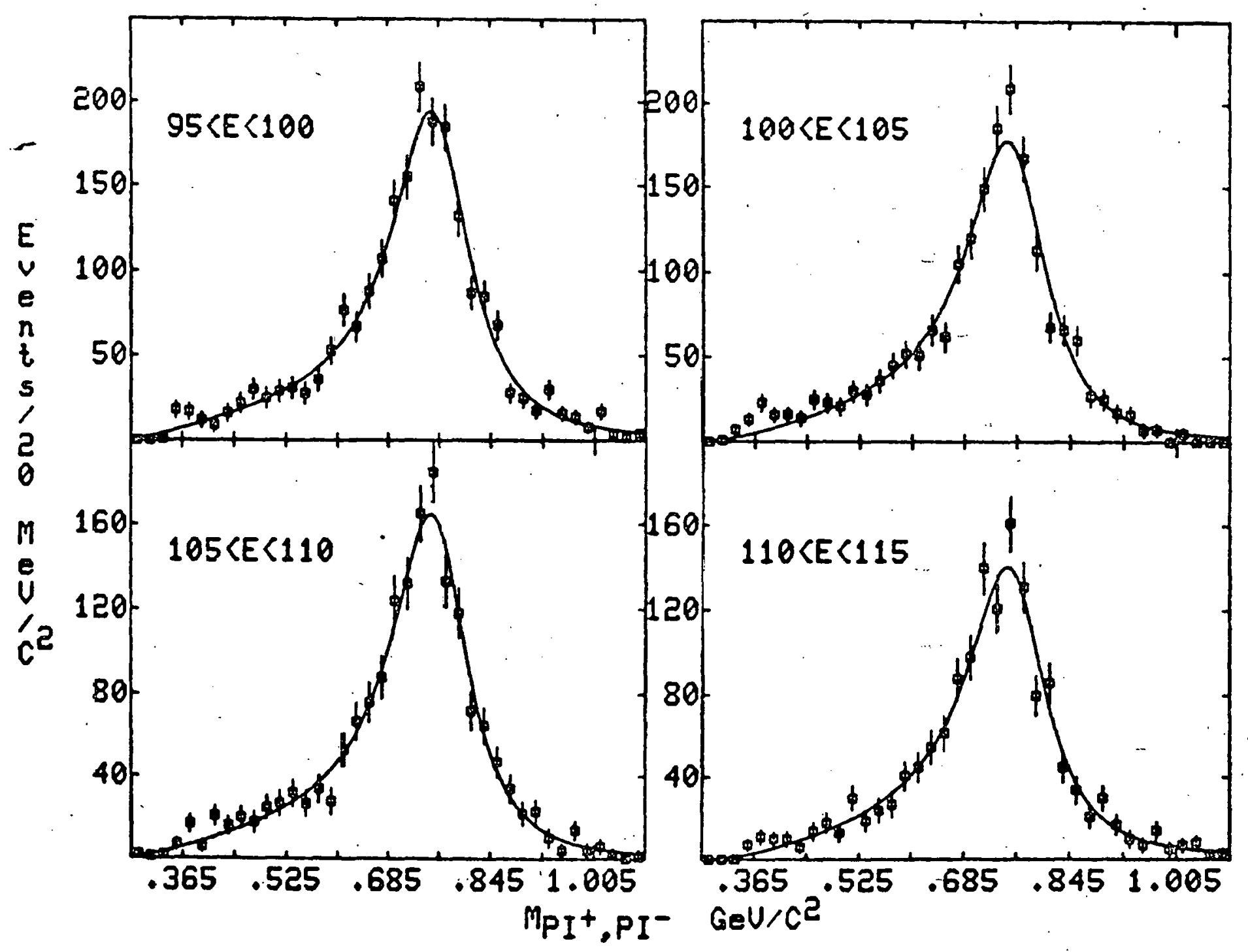

Figure 7.7 


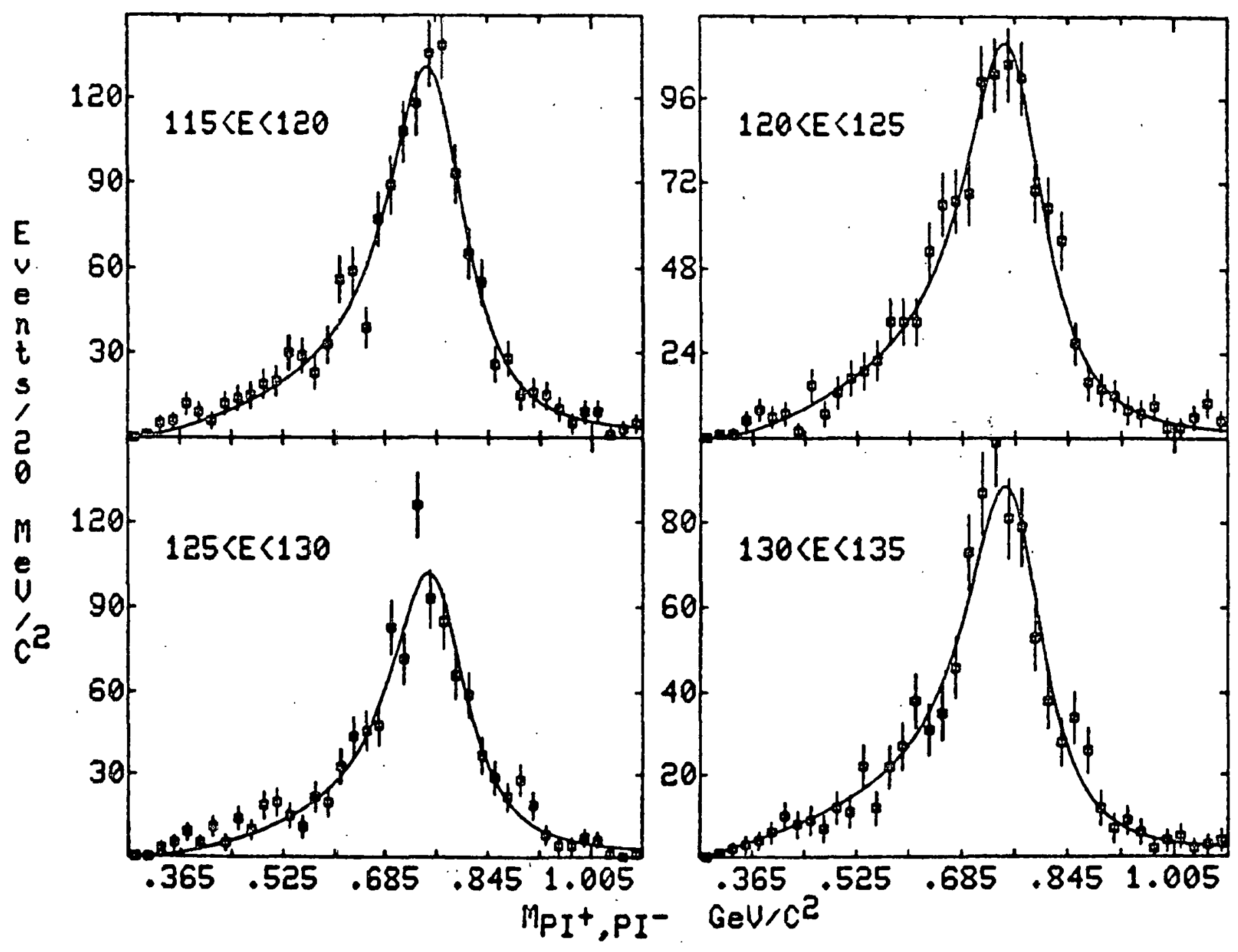

Figure 7.8 


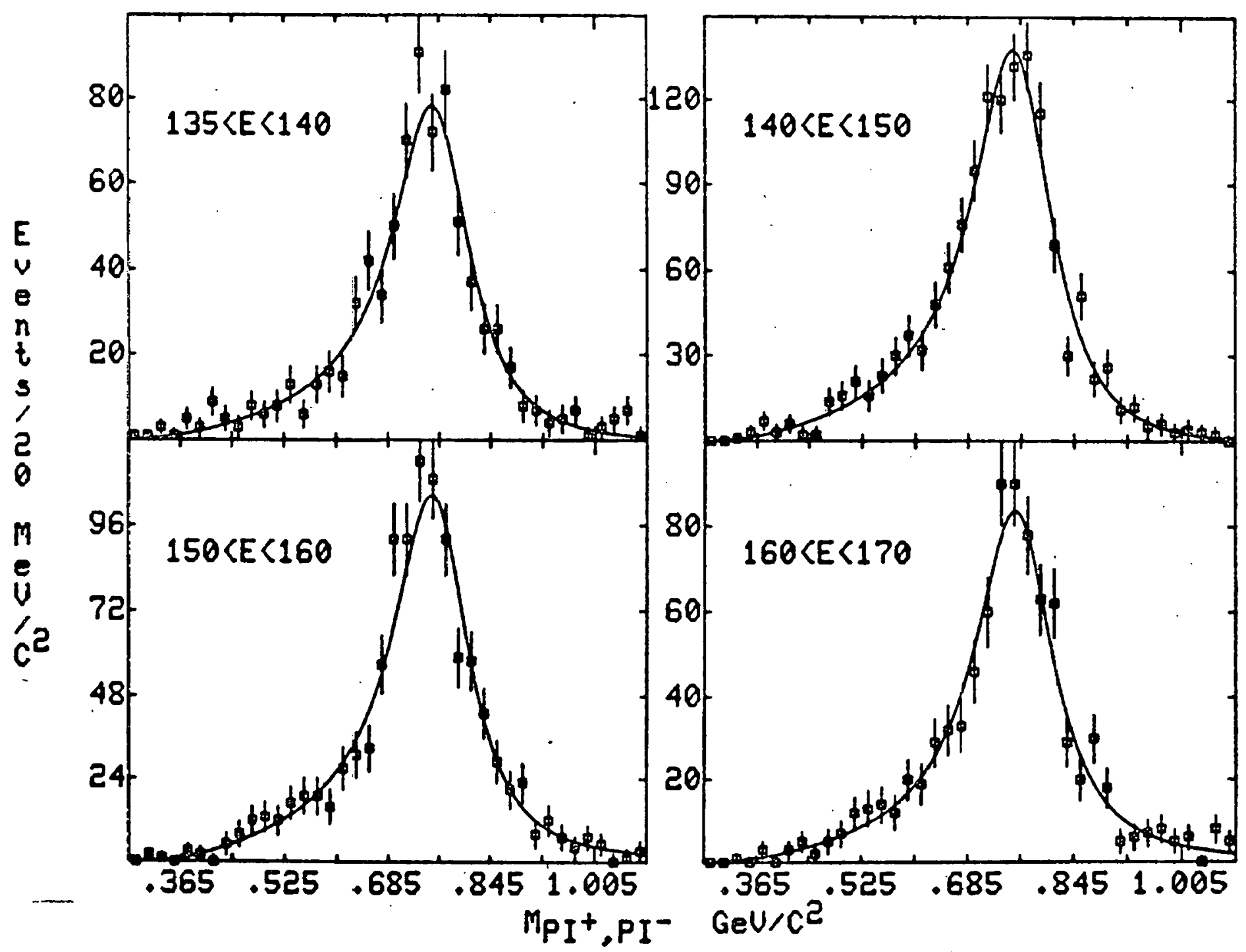

Figure 7.9 


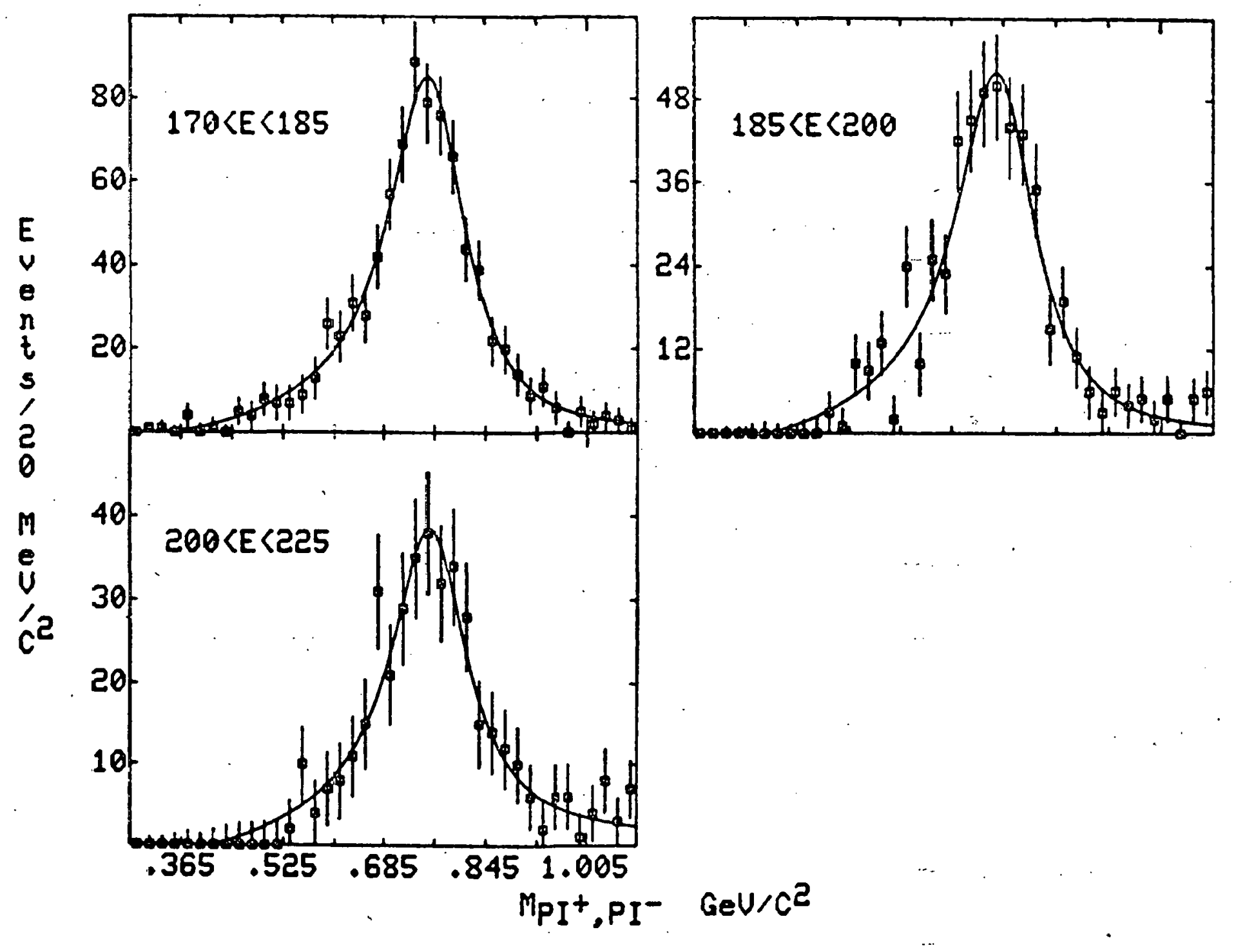

Figure 7.10 
obtained with equation 7.1 and the yields from these fits. The errors are the statistical errors from the fit only. Figure 7.11 shows a plot of the $e$ cross section as a function of energy.

This measurement is consistent with a very slow rise in the $\rho$ cross section of about 10 f from $\sim 10.5$ ub at low energy to -11.5 ub at high energy. With the known uncertainties in the calorimeter acceptance, this measurement is also consistent with a flat cross section of $10.5<\sigma<11 . \emptyset$ ub over the entire energy region. 
Table 7.1

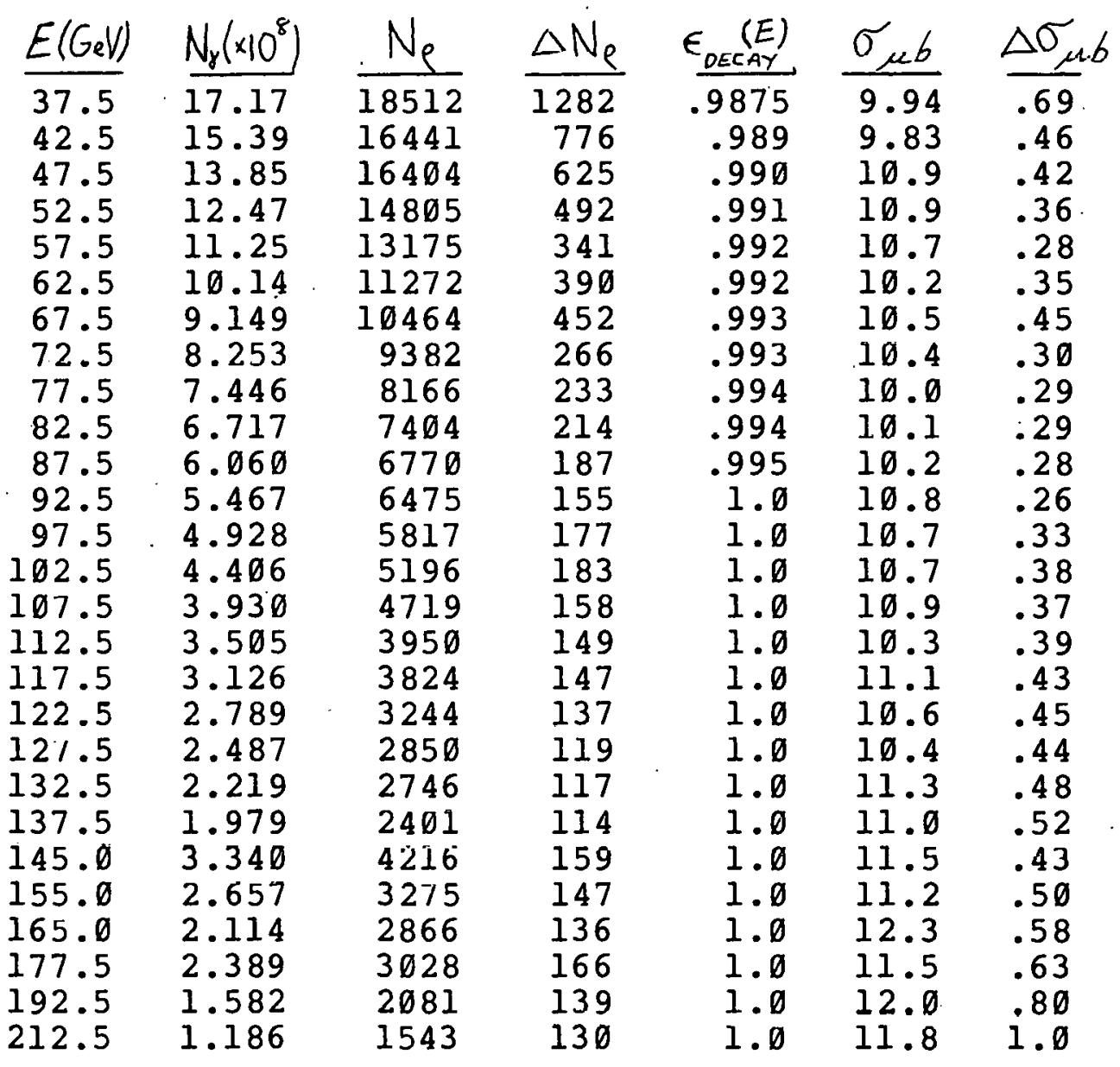




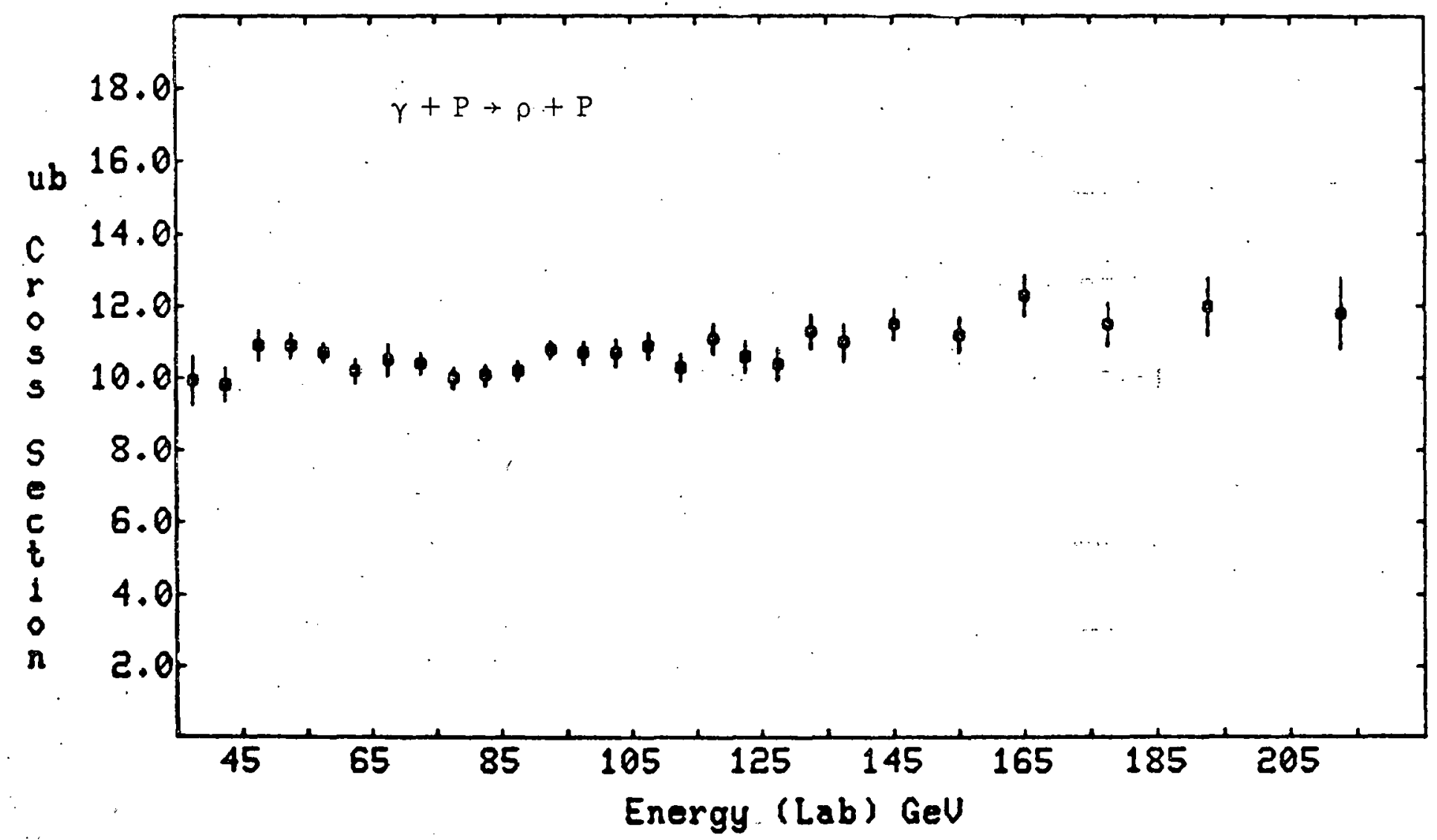

Figure 7.11 
The technique of calculating the calorimeter acceptance assumes that the acceptance is only a function of the total energy in the calorimeter and is not dependent on geometrical effects. To minimize any effects due to geometry, a 5" fiducial cut at the $\mathrm{HC}$ has been used in the analysis (see chapter 3). Leakage of hadronic energy near the edges of the $\mathrm{HC}$ is thereby minimized.

The geometric sensitivity was tested by measuring the cross section for events with varying energy asymmetry. The asymmetry parameter, A, was defined as:

$$
A=\frac{\left|P_{1}-P_{2}\right|}{p_{1}+P_{2}} ; P_{i}=\text { momentum of TRACKi }
$$

Symmetric and asymmetric events populate different geometrical regions of the HC. The data were divided into 3 data sets of roughly comparable statistics representing different regions of $A$. The same regions were defined in the Monte Carlo to properly measure the acceptance. Figures 7.12 to 7.14 show the cross sections computed in each region of $A$ overplotted with the cross section for the total sample. Statistical consistency is seen supporting the assumption that the total energy in the calorimeter is the dominant effect. 


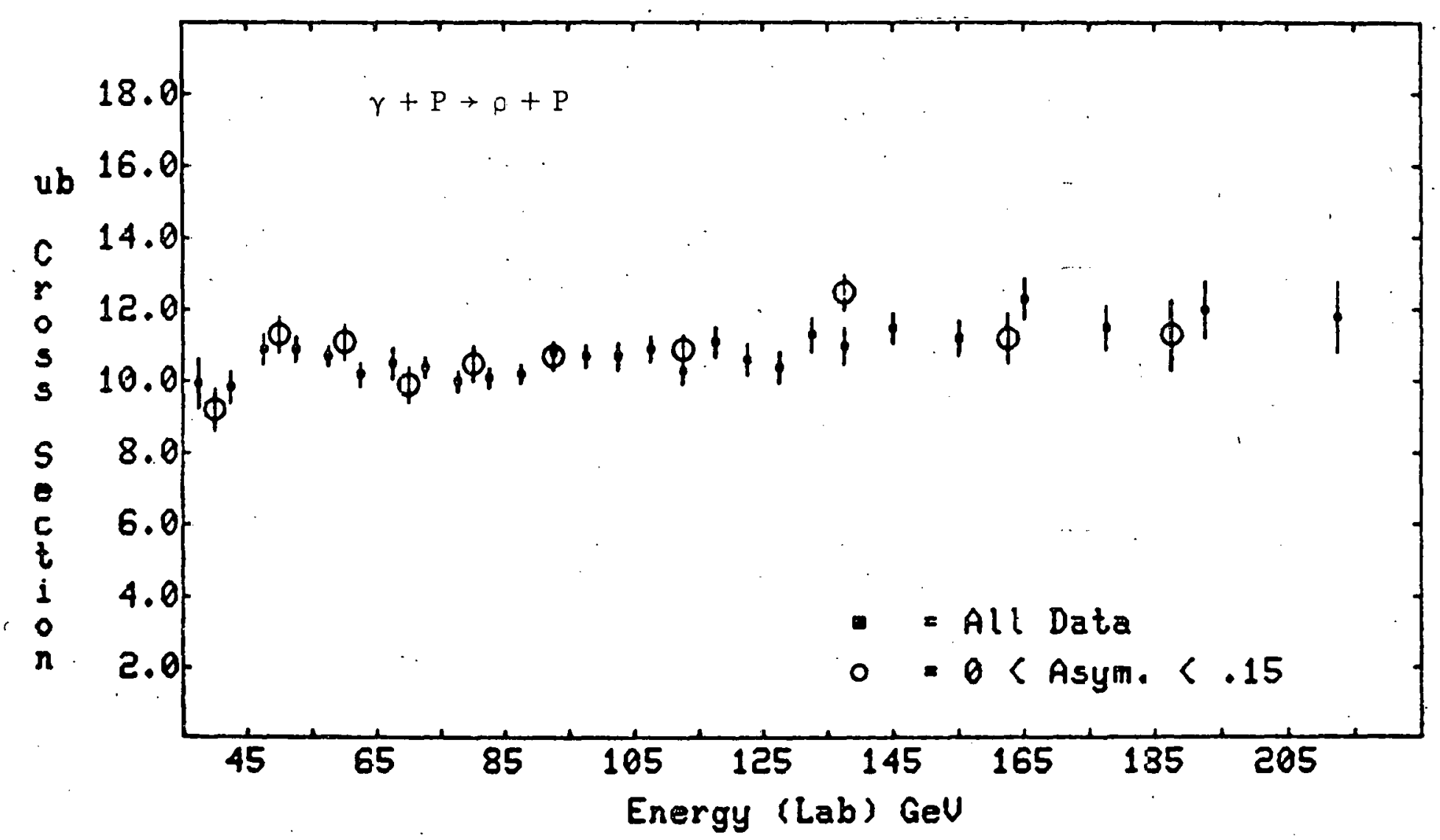

$\stackrel{\leftarrow}{N}$

Figure 7.12 


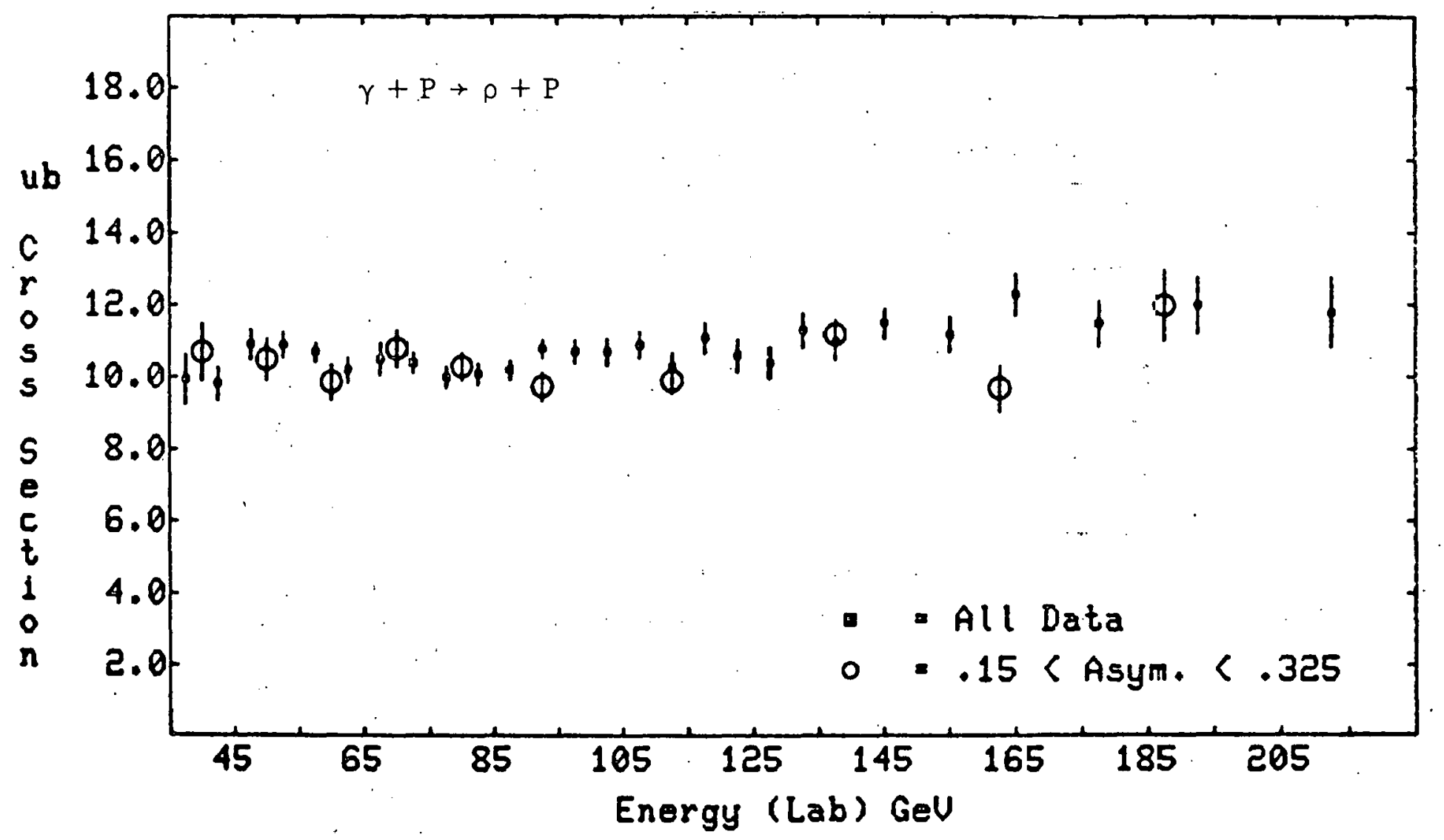

స్.

Figure 7.13 


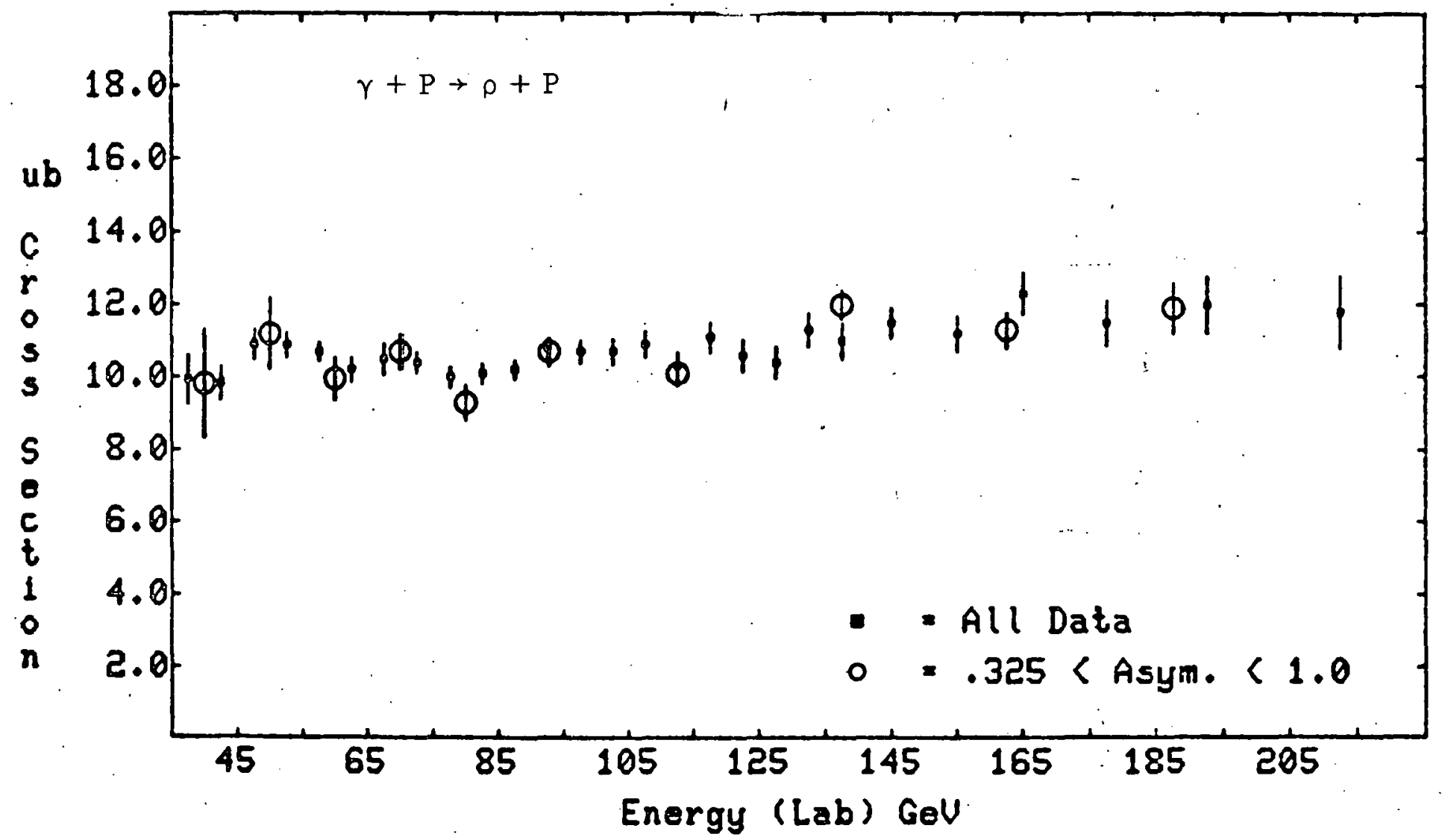


Section 3: $t$ Dependence for Elastic $\rho$ Photoproduction

The four-momentum transfer squared ( $t$ ) was computed for each event from equation 5.1. The yield in any given $t$ interval was determined by fitting the $k_{L}^{0}$ subtracted 2-pion mass distribution in that interval. The fitting function ( $f$ ) was: that of equation 7.4 with the added $t$ dimension:

$$
f\left(N_{\rho}, E, M_{\pi \pi}, t\right)=\frac{d N}{d M_{\pi \pi}}(e q .7 .1) \times A c c\left(E, M_{\pi \pi}, t\right)
$$

$\operatorname{Acc}\left(E, M_{\pi \pi}, t\right)$ is now the variation in the acceptance as a function of $2 \pi$ mass in a given energy and $t$ interval.

The bin widths for the $t$ distribution were chosen to be no less than twice the $t$ resolution in that region, as determined by the Monte carlo. The bin widths were made larger as more statistics were needed to define the mass distributions at higher energies and larger $t$.

The $t$ distribution was analyzed for the entire data set $(35<E<300)$ and in four different energy regions $(45-75,75-105,105-145,145-225)$. This was done so that the possible variation in the shape of the t-distribution and in $\mathrm{d} \sigma /\left.\mathrm{dt}\right|_{t=0}$ could be studied. 
The number of observed events can be related to the differential cross section as:

$$
N=N_{\gamma} \cdot L T \cdot n t \cdot A_{C C} \cdot \underset{\text { DECAY }}{\epsilon(E)} \cdot \epsilon_{M T}(t) \cdot \pi_{i} \epsilon_{i} \cdot B W \cdot \frac{d \sigma}{d t}(\gamma+p \rightarrow e+p)
$$

The variables are all as described in section 3 with one addition and one exception

$$
\begin{aligned}
\mathrm{BW}= & \mathrm{t} \text { bin width } \\
\epsilon_{M T}(t)= & \text { backround due to coherent production } \\
& \text { from the target end caps as a function } \\
& \text { of } t \text {; coherent production tends to be } \\
& \text { sharply peaked at low } t, \text { thus the } \\
& \text { correction is large there and very small } \\
& \text { elsewhere }
\end{aligned}
$$

To determine the correction $\epsilon_{M T}(t)$, events with a $2 \pi$ mass in the region $620<M_{\pi \pi}<92 \emptyset \mathrm{MeV}$ were studied that had a vertex at the T-counter (figure 3.1). Carbon is the predominant nucleus for coherent production from both the mylar target end caps and from the T-counter scintillator. Figure 7.15 shows the measured $t$ distribution at the T-counters. Note the large coherent peak at small $t$ and the emergence of the single nucleon slope at larger $t$. Both of these processes contribute background to $e$ production from hydrogen. The fitted function is the sum of two exponentials:

$$
A_{1} e^{-b_{1} t}+A_{2} e^{-b_{2} t}
$$

giving slope parameters:

$$
\begin{aligned}
& b_{1}=65+/-4(\mathrm{GeV} / \mathrm{c})^{-2} \\
& b_{2}=7.4+/-1.2(\mathrm{GeV} / \mathrm{c})^{-2}
\end{aligned}
$$




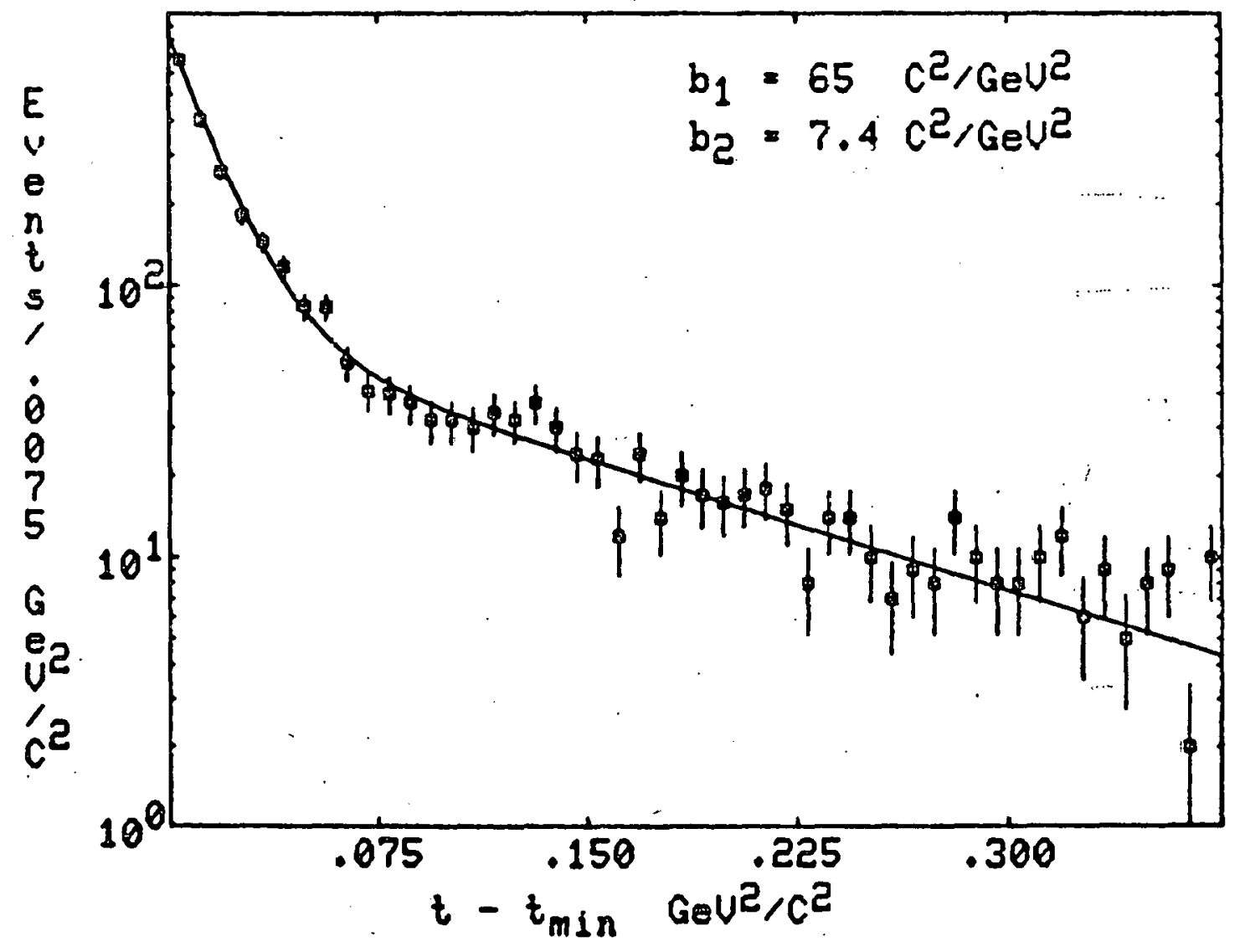


131

This distribution was normalized using the special empty target runs described in chapter 3 and chapter 7 , section 3 . It was determined there that the integrated backround was 3.3\%. Using the measured shape for the coherent background and knowing the absolute normalization, we obtain:

$$
f_{M T}=.033=\frac{\int_{0}^{\infty} N_{c}(t) d t}{N(0, \infty)}
$$

where

$$
\begin{aligned}
& N_{c}=\underset{\text { endcap contamination as a }}{\text { function of } t} \\
& N(0, \infty)=\# \text { events observed from the reg- } \\
& \text { lar data taking in the } t \text { inter- } \\
& \text { val } \emptyset<t<\infty
\end{aligned}
$$

Using the equation 7.7 to represent $N_{c}(t)$ we have:

$$
f_{M T}=\frac{A_{1} \int_{0}^{\infty}\left(e^{-b_{1} t}+R e^{-b_{2} t}\right) d t}{N(0, \infty)}
$$

where

$$
\mathrm{R}=\mathrm{A}_{2} / \mathrm{A}_{1}
$$

This equation can be integrated and solved for $A_{1}$ in terms of known parameters:

$$
A_{1}=\frac{f_{M T} \cdot b_{1} \cdot N(0, \infty)}{1+R\left(b_{1} / b_{2}\right)} \quad(7.9)
$$


For the contamination in any region $t_{1}<t<t_{2}$ we integrate $N_{c}(t)$ over that region and divide by the number of events observed in that region from the regular data runs:

$$
f\left(t_{1}, t_{2}\right)=\frac{A_{1} \int_{t_{1}}^{t_{2}}\left(e^{-b_{1} t}+R e^{-b_{2} t}\right) d t}{N\left(t_{1}, t_{2}\right)}
$$

Integrating this and substituting for ' $A_{1}$ ', we obtain the fractional contamination for any region $t_{1}<t<t_{2}$ :

$$
f\left(t_{1}, t_{2}\right)=\frac{f_{M T} \times N(0, \infty) \times\left[\left(e^{-b_{1} t_{1}}-e^{-b_{1} t_{2}}\right)+R\left[b_{1} / b_{2}\right]\left(e^{-b_{2} t_{1}}-e^{-b_{2} t_{2}}\right)\right]}{N\left(t_{1}, t_{2}\right) \times\left(1+R\left(b_{1} / b_{2}\right)\right)}
$$

All the parameters are known:

$$
\begin{aligned}
& \mathrm{f}_{M T}=.033 \\
& \mathrm{~b}_{1}=65 \mathrm{C}^{2} / G e V^{2} \\
& \mathrm{~b}_{2}=7.4 \mathrm{C}^{2} / G e V^{2} \\
& \mathrm{R}^{2}=\mathrm{A}_{2} / \mathrm{A}_{1}=69.55 / 740.3=.094
\end{aligned}
$$

The empty target correction is then

$$
\epsilon_{M T}(t)=\left[1-f\left(t_{1}, t_{2}\right)\right]^{-I}
$$

We can now compute the cross section as a function of $t$ making the proper corrections for coherent production from the target endcaps:

$$
\frac{d \sigma}{d t}(\gamma+\rho \rightarrow \rho+\rho)=\frac{9.101 \times 10^{5}}{B W} \times \frac{\epsilon_{M T}^{-1}(t)}{\epsilon_{D E C A Y}(E)} \times \frac{N_{\rho}}{N_{\gamma}} \frac{\mu b}{\left(G_{e V} / c\right)^{2}}
$$


Tables $7.2 \mathrm{~A}$ to $7.2 \mathrm{E}$ summarize the results for the 73 different regions of $E$ and $t$ considered. The errors quoted are the statistical errors only from the fits to the mass distribution.

Section 4: Fitting the $t$ distribution

The contribution to the $X^{2}$ in fitting a function $f(t)$ to an observed value $d \sigma / d t$ at some $t_{0}$ would ordinarily be:

$$
x^{2}=\left(\frac{d \sigma}{d t}\left(t_{0}\right)-f\left(t_{0}\right)\right)^{2} / \sigma^{2}
$$

where: $\sigma^{2}$ is the measurement error associated with $d \sigma / d t$

This is correct when $f(t)$ does not vary appreciably over the region of interest. Some of the $t$ bins in this study are wide compared to the probable variation in $\mathrm{d} \sigma / d t$. Therefore, the mean value theorem was used to determine the average value of $t$ in a bin $(t)$ :

$$
f(\bar{t})=\frac{1}{2 \Delta t} \times \int_{t_{0}-\Delta t}^{t_{0}+\Delta t} f\left(t^{\prime}\right) d t^{\prime}
$$

and the $X^{2}$ computed as:

$$
x^{2}=\left(\frac{d \sigma}{d t}\left(t_{0}\right)-f(\bar{t})\right)^{2} / \sigma^{2}
$$


Table 7.2A

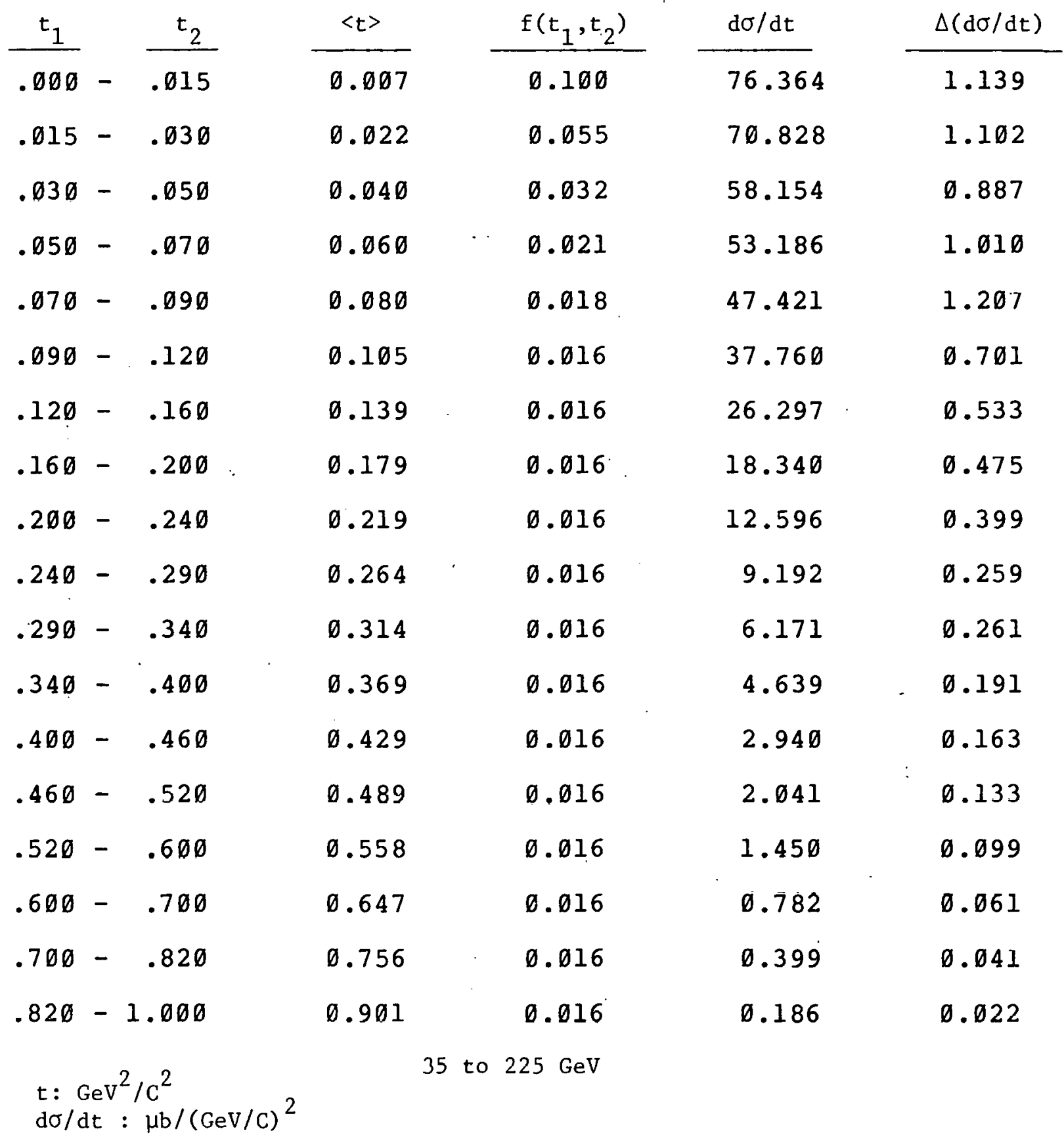


Table 7.2B

\begin{tabular}{|c|c|c|c|c|c|}
\hline $\mathrm{t}_{1}$ & $\mathrm{t}_{2}$ & $\langle\mathrm{t}\rangle$ & $f\left(t_{1}, t_{2}\right)$ & $\mathrm{d} \sigma / \mathrm{dt}$ & $\Delta(\mathrm{d} \sigma / \mathrm{d} \mathrm{t})$ \\
\hline.$\varnothing 0 \emptyset$ & -.015 & 0.007 & 0.100 & 76.783 & 3.273 \\
\hline .015 & -.030 & 0.022 & 0.055 & 71.275 & 2.219 \\
\hline .030 & -.050 & 0.040 & 0.032 & 59.010 & 1.740 \\
\hline .050 & -.070 & 0.060 & 0.021 & 57.277 & 2.139 \\
\hline .070 & -.090 & $\quad 0.080$ & 0.018 & 44.509 & 2.007 \\
\hline.$\varnothing 9 \emptyset$ & -.120 & 0.105 & 0.016 & 35.431 & 1.289 \\
\hline .120 & -.160 & 0.139 & 0.016 & 26.954 & 0.932 \\
\hline .160 & -.200 & 0.179 & 0.016 & 16.041 & 0.745 \\
\hline .200 & -.240 & $\emptyset .219$ & 0.016 & 12.160 & 0.617 \\
\hline .240 & -.290 & 0.264 & 0.016 & 9.479 & 0.446 \\
\hline .290 & -.340 & 0.314 & 0.016 & 6.070 & $\emptyset .388$ \\
\hline .340 & -.400 & 0.369 & 0.016 & 4.712 & 0.337 \\
\hline .400 & -.520 & 0.455 & 0.016 & 2.714 & 0.187 \\
\hline .520 & -.820 & 0.644 & $\emptyset . \emptyset 16$ & 1.178 & 0.077 \\
\hline $.82 \emptyset$ & -1.000 & 0.901 & 0.016 & 0.160 & 0.025 \\
\hline
\end{tabular}


Table $7.2 \mathrm{C}$

$$
\frac{t_{1}}{.000}-\frac{t_{2}}{.015}
$$$$
.015-.030
$$$$
.030-.050
$$$$
.050-.070
$$$$
.07 \emptyset-.09 \emptyset
$$$$
.090-.120
$$$$
.120-.160
$$$$
.160-.200
$$$$
.200-.240
$$$$
.240-.290
$$$$
.290-.340
$$$$
.340-.400
$$$$
.400-.520
$$$$
.520-.820
$$$$
.820-1.000
$$

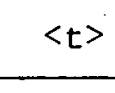

0.007

0.022

$\emptyset .040$

0.060

0.080

0.105

0.139

$\emptyset .179$

0.219

$\emptyset .264$

$\emptyset .314$

0.369

$\emptyset .455$

0.644

0.901

$\frac{f\left(t_{1}, t_{2}\right)}{0.100}$

0.055

0.032

0.021

0.018

0.016

0.016

0.016

0.016

$\emptyset .016$

0.016

0.016

$\emptyset .016$

0.016

0.016

75 to $105 \mathrm{GeV}$

\begin{tabular}{|c|c|}
\hline $\mathrm{d} \sigma / \mathrm{dt}$ & $\Delta(\mathrm{d} \sigma / \mathrm{d} t$ \\
\hline 77.276 & 2.273 \\
\hline 75.848 & 1.843 \\
\hline 59.939 & 1.442 \\
\hline 54.876 & 2.175 \\
\hline 48.244 & 2.040 \\
\hline 38.788 & 1.490 \\
\hline 27.357 & 1.008 \\
\hline 17.789 & 0.783 \\
\hline 12.587 & 0.694 \\
\hline 9.915 & 0.560 \\
\hline 6.730 & 0.406 \\
\hline 4.367 & 0.313 \\
\hline 2.505 & $\emptyset .169$ \\
\hline 0.849 & 0.063 \\
\hline 0.164 & 0.030 \\
\hline
\end{tabular}


Table 7.2D

\begin{tabular}{|c|c|c|c|c|c|}
\hline$t_{1}$ & $t_{2}$ & $\langle t\rangle$ & $f\left(t_{1}, t_{2}\right)$ & $\mathrm{d} \sigma / \mathrm{dt}$ & $\Delta(\mathrm{d} \sigma / \mathrm{d} t)$ \\
\hline.$\emptyset \emptyset \emptyset$ & -.015 & 0.007 & 0.100 & 92.067 & 2.616 \\
\hline .015 & -.030 & 0.022 & 0.055 & 75.251 & 2.772 \\
\hline .030 & -.050 & $\emptyset .040$ & 0.032 & 64.939 & 2.171 \\
\hline .050 & -.070 & 0.060 & 0.021 & 57.273 & 2.545 \\
\hline .070 & -.090 & $\emptyset . \emptyset 8 \emptyset$ & .0 .018 & 54.978 & 2.656 \\
\hline .090 & $-\quad 120$ & 0.105 & 0.016 & 41.361 & 1.731 \\
\hline .120 & -.160 & 0.139 & 0.016 & 27.723 & 1.206 \\
\hline .160 & -.200 & 0.179 & 0.016 & 21.333 & 1.072 \\
\hline .200 & -.240 & 0.219 & 0.016 & 14.634 & 0.928 \\
\hline .240 & $-\quad .290$ & 0.264 & 0.016 & 9.564 & 0.602 \\
\hline .290 & -.370 & 0.328 & 0.016 & 6.699 & 0.423 \\
\hline .370 & $-\quad .520$ & 0.438 & 0.016 & 2.913 & 0.206 \\
\hline .520 & $-\quad .820$ & 0.644 & 0.016 & 0.690 & 0.047 \\
\hline .820 & -1.000 & 0.901 & 0.016 & 0.330 & 0.069 \\
\hline
\end{tabular}


Table 7.2E.

\begin{tabular}{|c|c|c|c|c|c|}
\hline$t_{1}$ & & $t_{2}$ & $\langle t\rangle$ & & $f\left(t_{1}, t_{2}\right)$ \\
\hline .000 & - & .015 & 0.007 & & 0.100 \\
\hline .015 & - & .030 & 0.022 & & 0.055 \\
\hline .030 & - & .050 & 0.040 & & 0.032 \\
\hline .050 & - & .070 & 0.060 & " & 0.021 \\
\hline .070 & - & .090 & 0.080 & & $\emptyset .018$ \\
\hline .090 & - & .120 & 0.105 & & 0.016 \\
\hline .120 & - & .160 & 0.139 & & 0.016 \\
\hline .160 & - & .220 & 0.189 & & 0.016 \\
\hline .220 & - & .340 & 0.275 & & 0.016 \\
\hline .340 & - & .600 & 0.449 & & 0.016 \\
\hline .600 & & .000 & 0.756 & & $\emptyset .016$ \\
\hline & & & & 45 & $225 \mathrm{GeV}$ \\
\hline
\end{tabular}

$\begin{array}{cc}\frac{\mathrm{d} \sigma / \mathrm{d} t}{93.845} & \frac{\Delta(\mathrm{d} \sigma / \mathrm{d} t)}{4.526} \\ 86.004 & 4.35 \emptyset \\ 75.708 & 3.766 \\ 62.589 & 3.888 \\ 60.441 & 4.757 \\ 46.841 & 3.149 \\ 27.714 & 2.010 \\ 21.078 & 1.314 \\ 9.433 & \emptyset .599 \\ 2.870 & 0.216 \\ 0.416 & \emptyset .059\end{array}$


It was found that the $t$ distribution in all energy regions could be described by an exponential function with a quadratic term:

$$
f(t) \propto \exp \left(-b t+c t^{2}\right)
$$

Table 7.3 summarizes the results for the slope parameters $b$ and $c$ in different energy regions. There is no evidence for any systematic variation with energy. The best values for $b$ and $c$ were taken from the fit to the $t$ distribution integrated over all energies:

$$
\begin{aligned}
& \mathrm{b}=8.82+/-.04(\mathrm{GeV} / \mathrm{c})^{-2} \\
& \mathrm{c}=2.3 \emptyset+/-.08(\mathrm{GeV} / \mathrm{c})^{-4}
\end{aligned}
$$

Figure 7.16 shows this distribution and the fitted function plotted over it. The $t$ distribution can be represented by a single slope exponential function for $t<.24(\mathrm{GeV} / \mathrm{c})^{2}$ with slope parameter :

$$
\mathrm{b}=8.3+/-.08(\mathrm{GeV} / \mathrm{c})^{-2} ; \quad t<.24(\mathrm{GeV} / \mathrm{c})^{2}:
$$

Section 5: Measurement of $\mathrm{d} \sigma /\left.\mathrm{d} t\right|_{t=0}$

The cross sections in each energy region were fit by freezing the slope parameters at the values given in eq. 7.13 and allowing only the absolute normalization to vary. The shape of the $t$ distributions is extremely well described by these values of $b$ and $c$ in all energy regions. Figures 7.17 to 7.20 show these fits to the $t$ distribution in each energy region. 


\section{Table 7.3}

\begin{tabular}{|c|c|c|}
\hline$E(\mathrm{GeV})$ & $\mathrm{b}(\mathrm{GeV} / \mathrm{C})^{-2}$ & c. $(\mathrm{GeV} / \mathrm{C})^{-4}$ \\
\hline $35-225$ & $8.82+/-.04$ & $2.30+/-.08$ \\
\hline $45-75$ & $9.03+/-.17$ & $2.72+1-.24$ \\
\hline $75-105$ & $8.91+/-.05$ & $2.36+/-.15$ \\
\hline $105-145$ & $9.04+/-.25$ & $2.27+/-.44$ \\
\hline $145-225$ & $9.26+1-.32$ & $2.57+1-.55$ \\
\hline
\end{tabular}




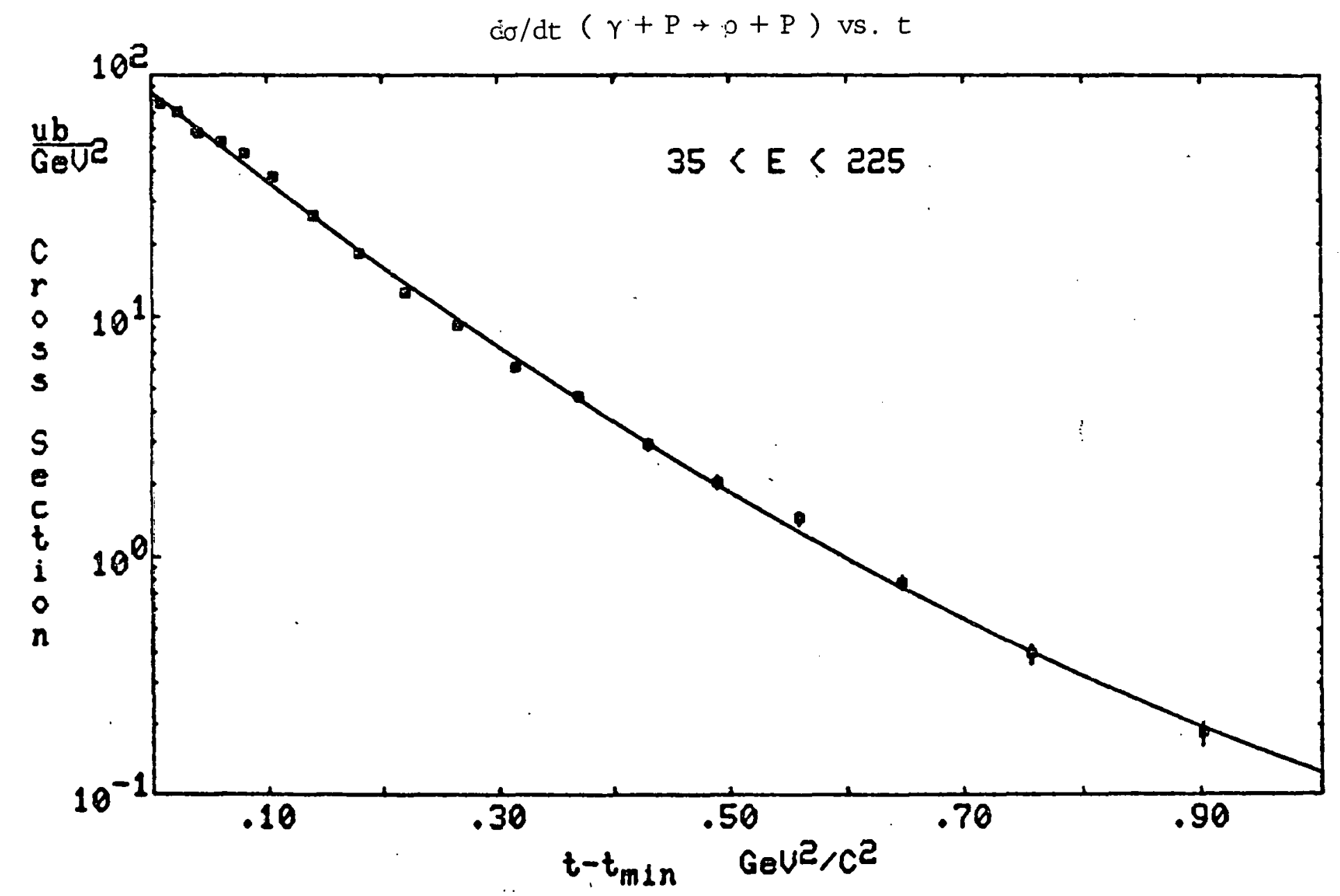

Figure 7.16 


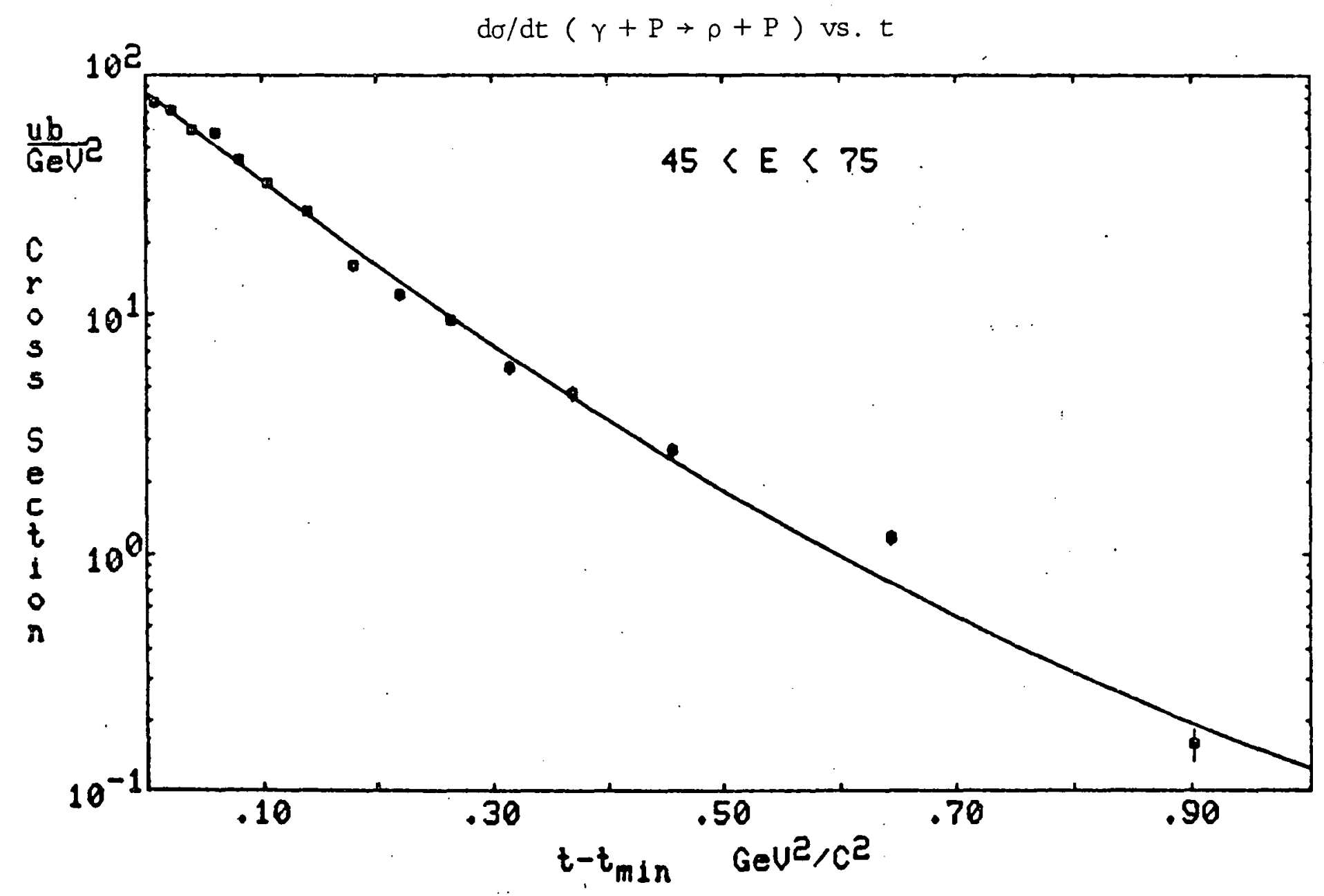

Figure 7.17 


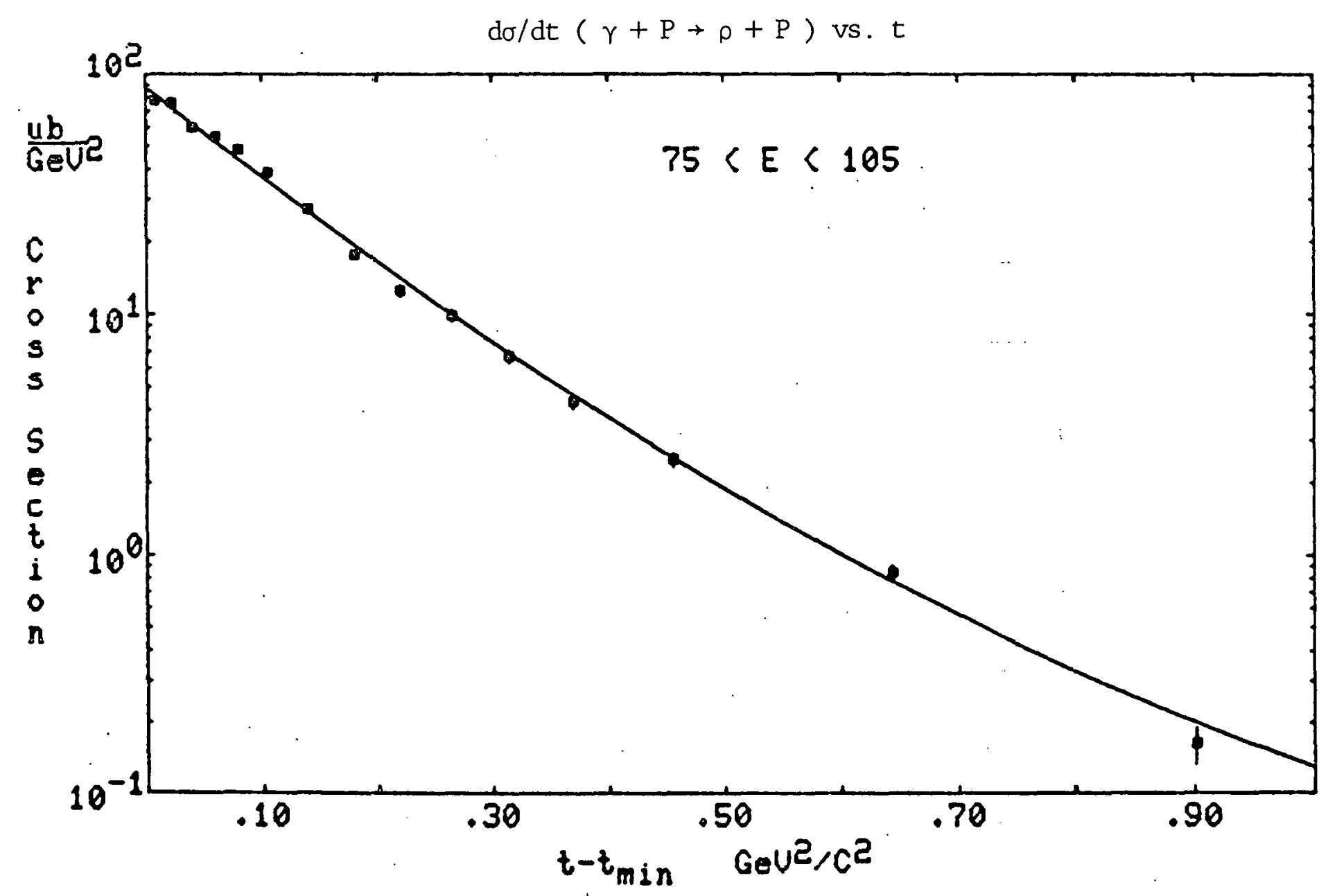

Figure 7.18 


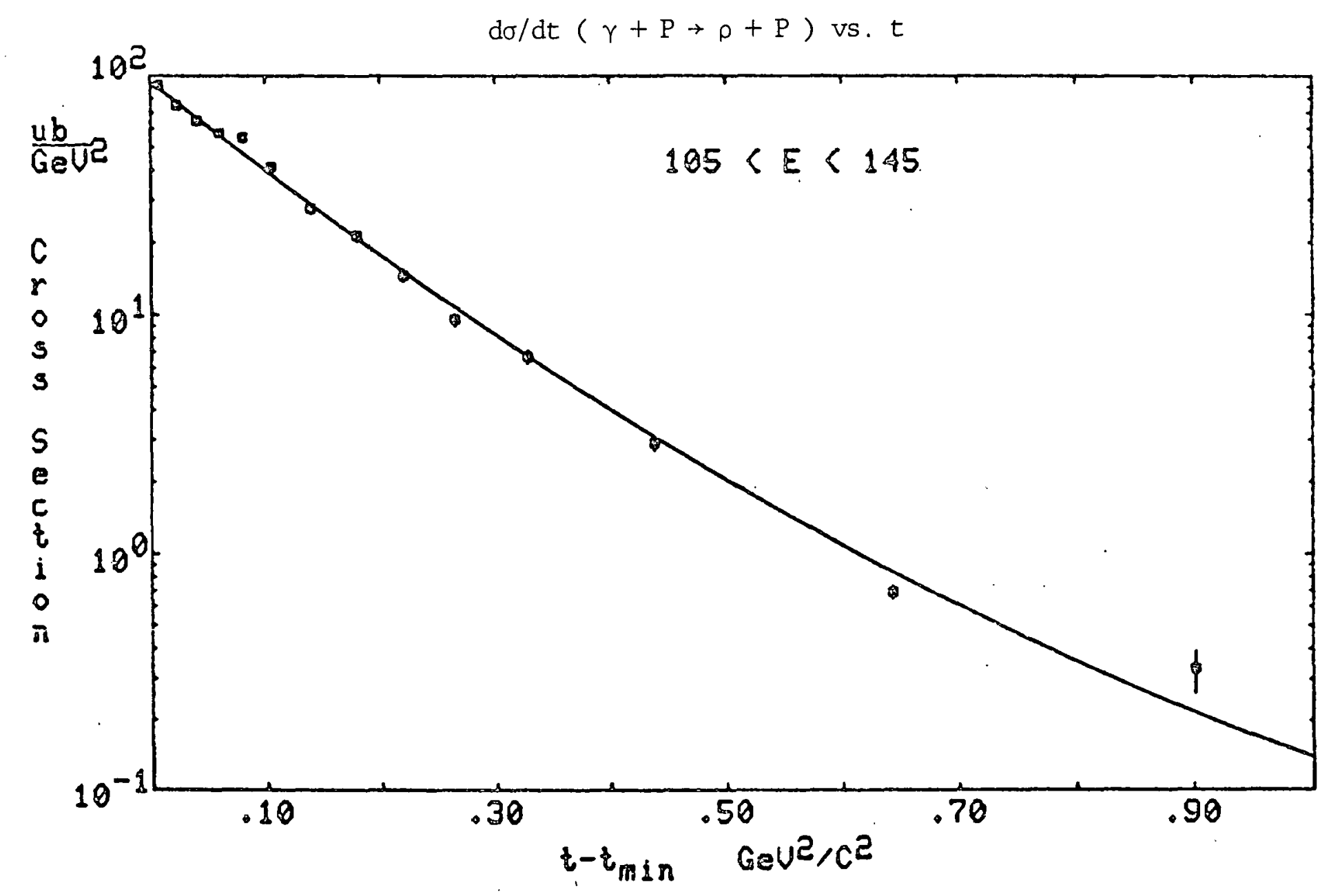

Figure 7.19 


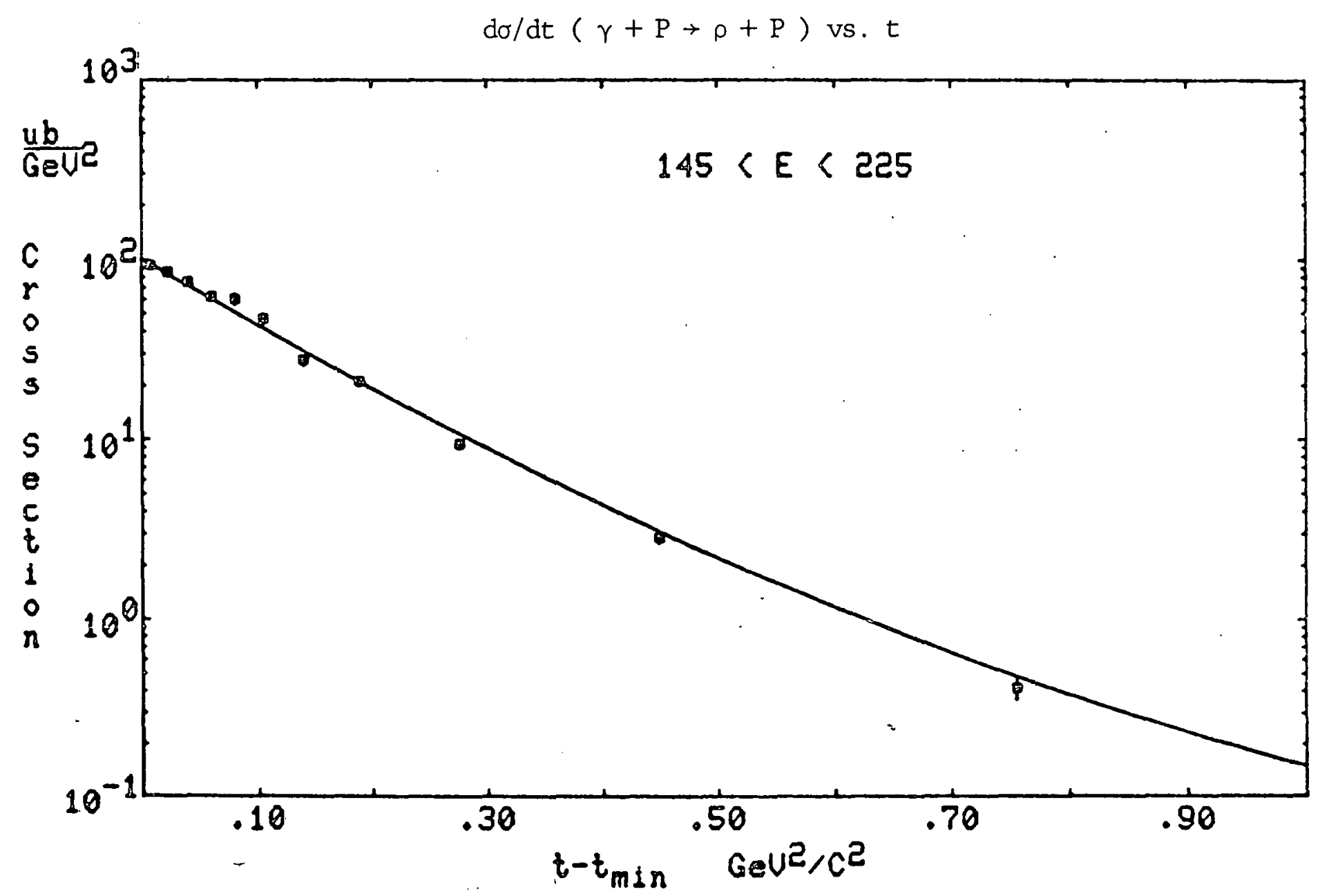


Table 7.4 summarizes the resultant values for $d \sigma / d t(t=\emptyset)$ :

\section{Table 7.4}

$\begin{array}{ll}\underline{\mathrm{E}(\mathrm{GeV})} & \underline{\mathrm{d} \sigma / \mathrm{dt}} \quad \underline{\mathrm{ub} /\left(\mathrm{GeV}^{2} / \mathrm{c}^{2}\right)} \\ 61+/-8 & 85.0+/-.97 \\ 89+/-8.5 & 87.4+/-.91 \\ 122+/-11 & 94.1+/-1.2 \\ 171+/-20 & 101.5+/-1.9\end{array}$

Section 6: Diffractive Inelastic $\rho$ production

The fraction of diffractive $\rho$ production that is inelastic was measured for $\emptyset<t<.9 \mathrm{GeV}^{2} / \mathrm{c}^{2}$. The inelastic events were those that were inconsistent with elastic production as determined by the analysis of the recoil detector (see chapter 5 and appendix D). Events with a di-pion mass within $150 \mathrm{MeV}$ of the $\rho$ mass were used. : A tight target cut $(-262.5 "<z<-256.5 ")$ was made to ensure acceptance in the $\mathrm{RC}$ fiducial volume and to eliminate any contamination from the target end caps. The same criteria were applied to the $K_{L}^{0}$ data and the necessary subtractions were made. For $t>.15 \mathrm{GeV}^{2} / \mathrm{c}^{2}, 77 \%$ of the $\mathrm{K}_{L}^{0}$ data were inelastic compared with $34 \%$ for the photoproduced $\rho$ data. 
The fraction of diffractive $\rho$ production that is inelastic as a function of $t, f(t)$, is

$$
f(t)=\frac{N_{i}^{\top}(t)}{N_{i}^{\top}(t)+N_{e}^{\top}(t)}
$$

where

$$
\begin{aligned}
& N_{i}^{\top}(t)=\text { the "true" number of inelastic diffractive events } \\
& N_{e}^{\top}(t)=\text { the "true" number of elastic diffractive events }
\end{aligned}
$$

The observed number of inelastic events is contaminated with elastic events that have been misidentified by RC6. A Monte Carlo study was made to determine the fraction of true elastic events, $\varepsilon$, that are misidentified as a function of $t$. The resulting correction had a maximum value of 6.58 for $t$ in the threshold region for the proton to exit the $\mathrm{LH}_{2}$ target and was typically $2 \%$ to $3 \%$ otherwise. Expressing the true quantities in tems of the observed quantities, we obtain:

$$
\begin{aligned}
N_{e}^{\top}(t) & =\frac{N_{e}^{0}(t)}{(1-\varepsilon)} \\
N_{i}^{\top}(t) & =N_{i}^{0}-\varepsilon \times N_{e}^{\top}(t) \\
& =N_{i}^{0}(t)-\frac{\varepsilon}{(1-\varepsilon)} N_{e}^{0}(t)
\end{aligned}
$$


The superscript "o" implies an observed quanitiy. Plugging into equation 7.14 for $\mathrm{N}_{e}^{\top}$ and $\mathrm{N}_{i}^{\top}$ obtains:

$$
f(t)=\frac{N_{i}^{0}(t)-\frac{\varepsilon}{(1-\varepsilon)} N_{e}^{0}(t)}{N_{i}^{0}(t)+N_{e}^{0}(t)}
$$

The fraction of diffractive $\rho$ events that is inelastic, $f(t)$, is plotted as a function of $t$ in figure 7.21. The curve is a fit to a. function of the form:

$$
f(t)=\left[1+R_{0} x \exp \left(-b_{1} t+c_{1} t^{2}-b_{2} t\right)\right]^{-1}
$$

where:

$$
\begin{aligned}
& b_{1}=8.22(\mathrm{GeV} / \mathrm{c})^{-2} \\
& c_{1}=2.3 \emptyset(\mathrm{GeV} / \mathrm{c})^{-4}\left\{\begin{array}{l}
\text { Fixed from the } \\
\text { measurement of the } \\
\text { elastic t-distribu- } \\
\text { tion (see eq. } 7.13)
\end{array}\right. \\
& \mathrm{b}_{2}=\begin{array}{l}
\text { slope parameter for the inelastic } b_{2} t \\
\text { distribution assuming a form e }-b_{2}
\end{array} \\
& \mathrm{R}_{0}=\begin{array}{l}
\text { ratio of elastic/inelastic events } \\
\text { at } t=\emptyset
\end{array}
\end{aligned}
$$

From the best fit we obtain an inelastic slope parameter of:

$$
\mathrm{b}_{2} \quad=4.0+/-.2(\mathrm{GeV} / \mathrm{c})^{-2}
$$

For the ratio of elastic/inelastic events at $t=\emptyset$ we obtain :

$$
\mathrm{R}_{0}=8.1+/-0.2
$$

The fraction of diffractive $\rho$ events that is inelastic is seen to be in the neighborhood of $10 \%$ near $t=0$ and slowly rising to $55 \%$ at $t=.8 \mathrm{GeV}^{2} / \mathrm{c}^{2}$. A previous measurement [24] at low energy $(E=8.5 \mathrm{GeV})$ determined the fraction of 


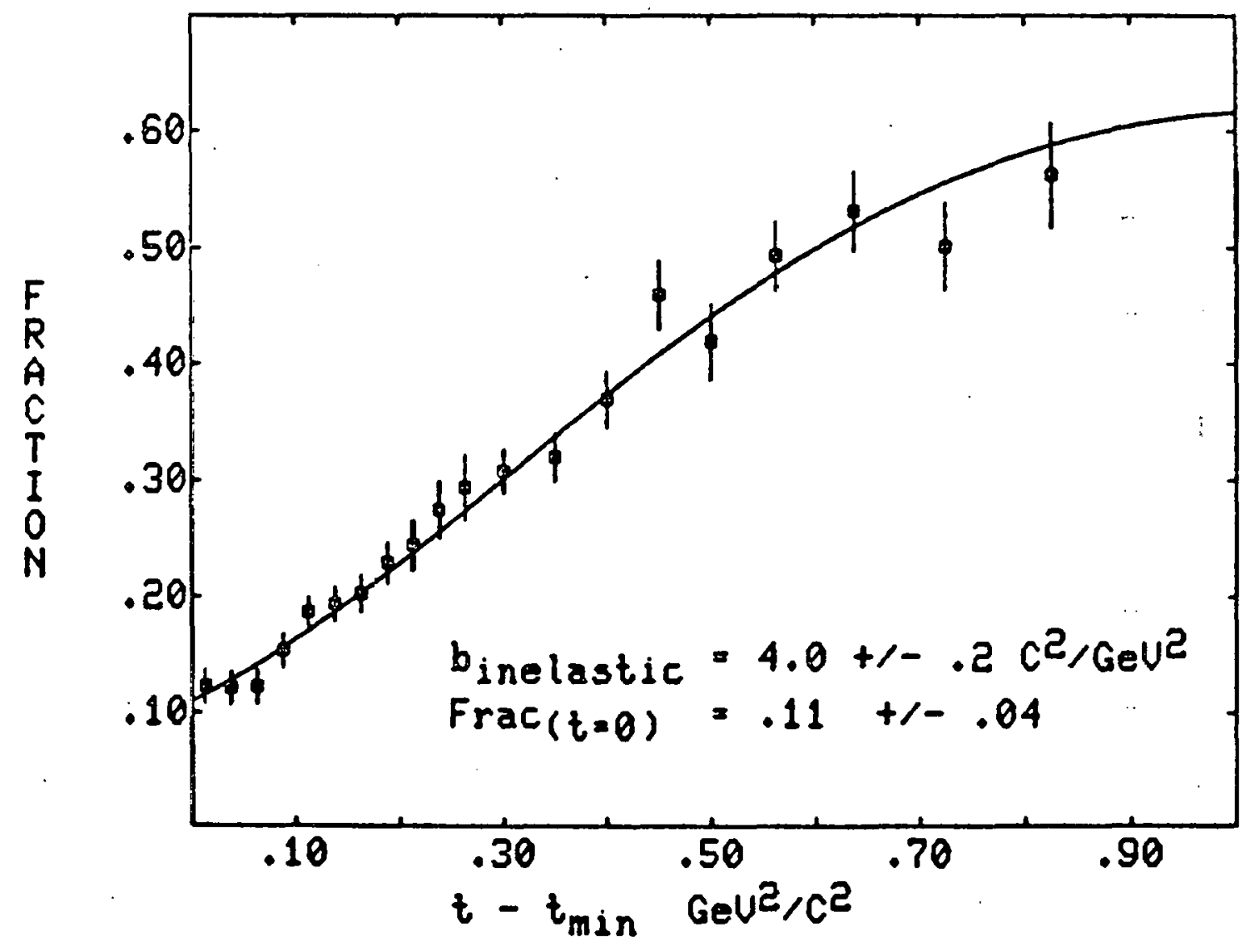


diffractive $\rho$ events that are inelastic to be $\sim 5 \%$ near $t=\emptyset$. rising to 258 at $t=.4 \mathrm{GeV}^{2} / \mathrm{c}^{2}$. We measure the fraction to be $35 \%$ at $t=.4 \mathrm{GeV}^{2} / \mathrm{c}^{2}$. 


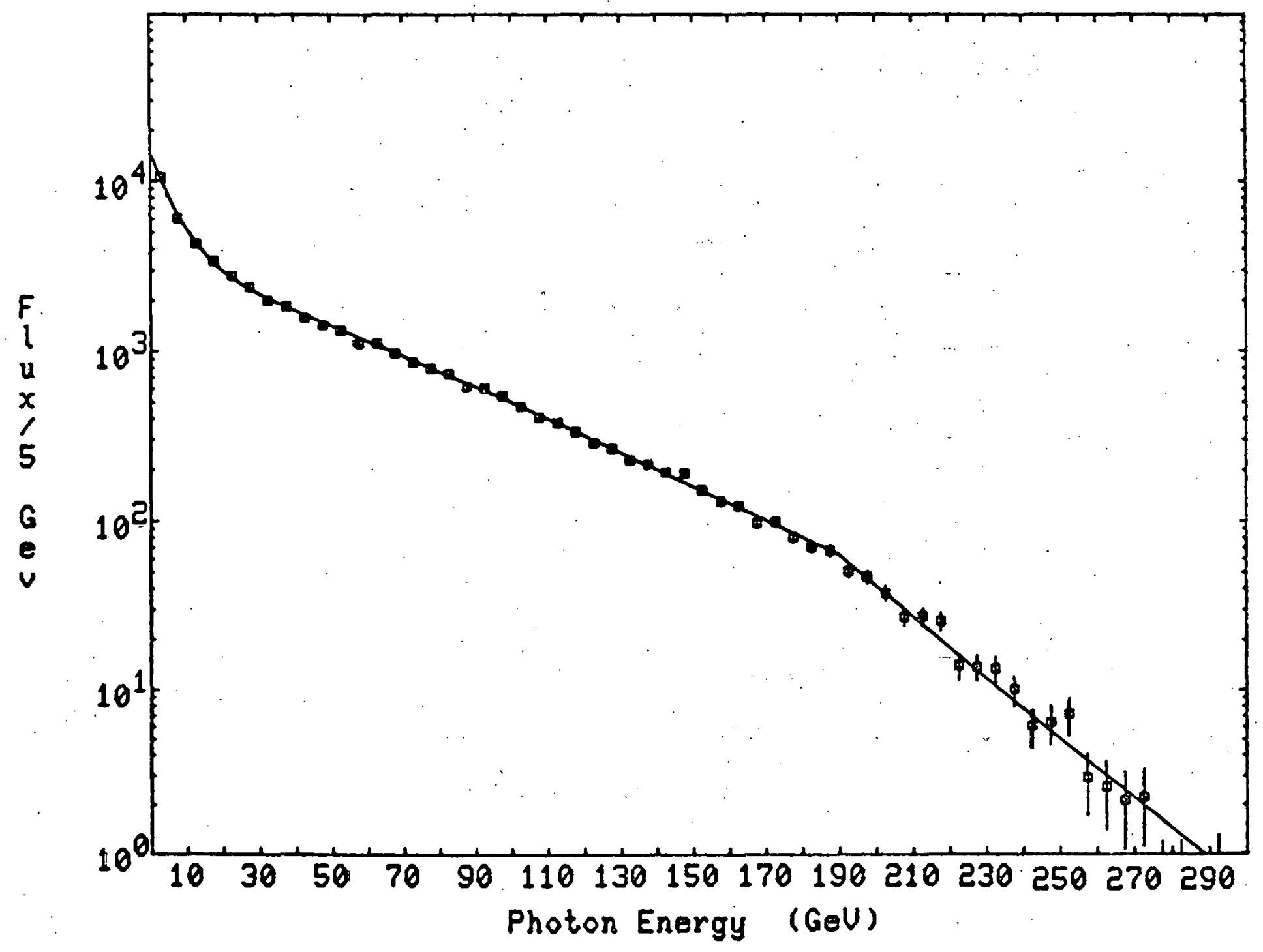

Figure 7.22 


\section{2 .}

\section{Chapter VIII}

\section{$\phi$ Analysis}

Elastic $\phi$ photoproduction, $\gamma+\mathrm{p} \rightarrow \phi\left(\rightarrow \mathrm{k}^{+} \mathrm{K}^{-}\right)+\mathrm{p}$, has been observed for incident photon energies from 35 to 165 Gev. The $t$ distribution has been measured from $\emptyset$ to 1 $\mathrm{GeV}^{2} / \mathrm{c}^{2}$. The fraction of diffractive $\phi$ production that is inelastic has been determined. A total of 800 photoproduced 2-track events with a $\mathrm{K}^{+} \mathrm{K}^{-}$invariant mass in the region of the $\phi$ were analyzed.

The $\phi$ is a narrow resonance with a natural width of $4.1+/-.2 \mathrm{MeV}[25]$. The measured width from this experiment, due to resolution, is $7.5 \mathrm{MeV}$. Therefore, the shape of the observed resonance is not properly described by either a Breit-Wigner distribution (too narrow) or by a Gaussian distribution (too broad). To fit the observed resonance, we have used a "smeared" relativistic p-wave Breit-Wigner lıne shape.

$$
\frac{d N}{d M_{K K}}=A_{0} \int_{M-3 \sigma}^{M+3 \sigma} \frac{M_{0}^{2} \Gamma\left(M^{\prime}\right)}{\left(M_{0}^{2}-M^{\prime 2}\right)^{2}+M_{0}^{2} \Gamma^{2}\left(M^{\prime}\right)} \times \frac{e^{-\left(M-M^{\prime}\right)^{2} / 2 \sigma^{2}}}{\sqrt{2 \pi \sigma^{2}}} d M^{\prime}+B G
$$


where $\Gamma(M)$ is the mass dependent width:

$$
\begin{aligned}
& \Gamma(M)=\Gamma_{0}\left\{\frac{M^{2}-4 M_{k}^{2}}{M_{0}^{2}-4 M_{k}^{2}}\right\}^{3 / 2} \\
& M_{0}=\text { resonance mass } \\
& \Gamma_{0}=\text { natural width } \\
& \sigma=\text { experimental mass resolution, which is } \\
& \text { determined from the Monte Carlo } \\
& \text { BG = polynomial for background (which is } \\
& \text { mainly the reflection of low mass } \\
& \text { di-pions) }
\end{aligned}
$$

In the fit to the entire $\phi$ sample, $\Gamma_{0}$ was fixed at 4.1 $\mathrm{MeV}$ and the mass resolution, $\sigma$, at $2 \mathrm{MeV}$. The resonance mass and the overall normalization were allowed to vary. Figure 8.1 shows the entire $\mathrm{K}^{+} \mathrm{K}^{-}$invariant mass distribution and the fit using equation 8.1. The peak is very accurately described by this technique. From the. fit we obtain a resonance mass of:

$$
\mathrm{M}_{0}=1019.6+/-.17 \mathrm{MeV}
$$

where a $0.2 \mathrm{MeV}$ net measurement offset, determined by the Monte Carlo, has been subtracted from $M_{0}$.

In fitting the data in different energy regions, $M_{0}$ was fixed at the value determined from the fit to the entire sample. The mass resolution, $\sigma$, was determined separately in each energy region by the Monte Carlo and then fixed in the 


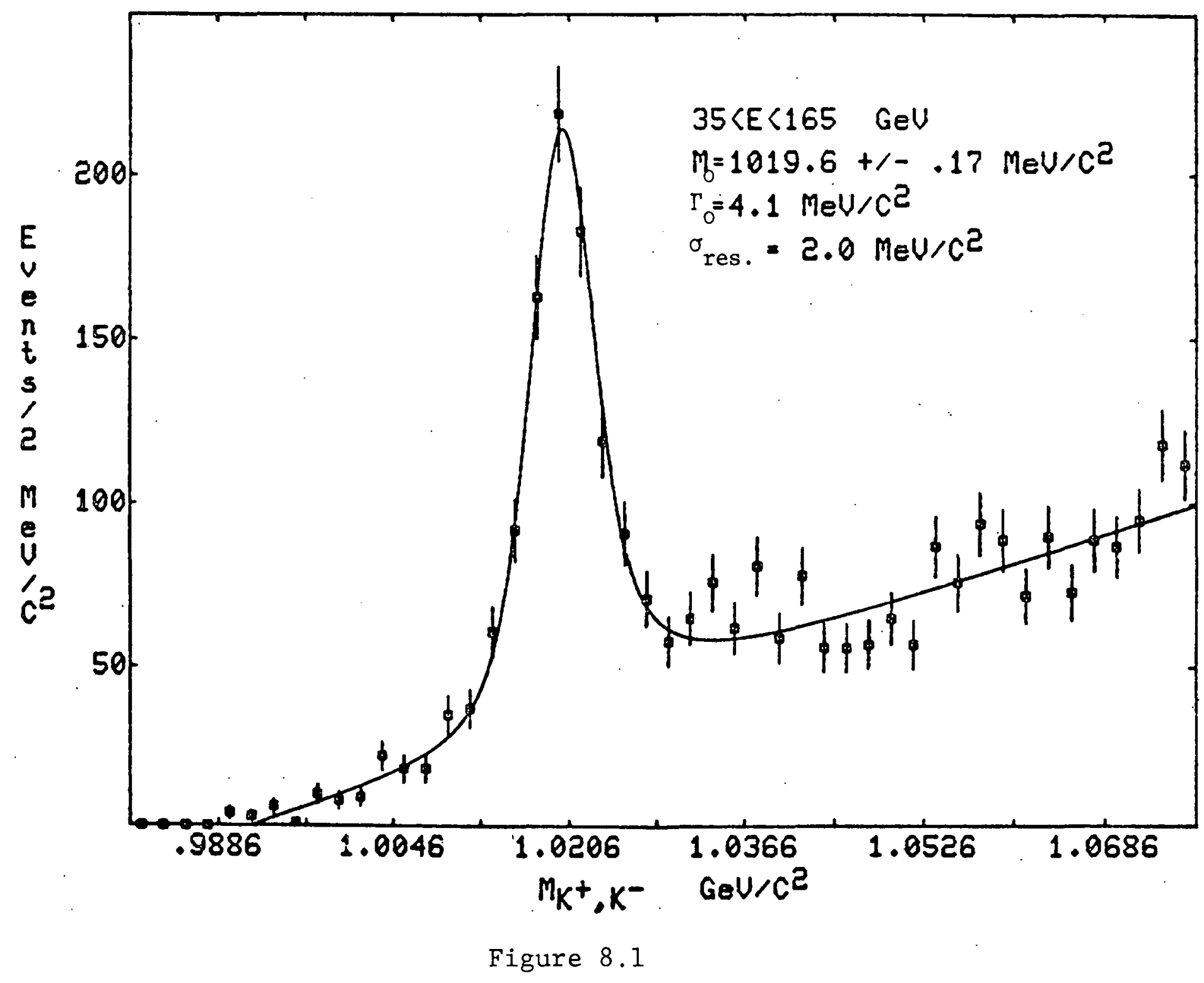

$\stackrel{n}{*}$ 
fit. Only the background distribution and the overall normalization were allowed to vary in these fits.

The technique just described for fitting the mass distribution has three principal advantages. First, it is entirely self consistent at all energies, with the resonance mass, natural width and mass resolution being fixed. Secona, it eliminates the $20 \%$ systematic difference in the yield obtained by comparing the yield from a Gaussian with the yleld from a Breit-wigner. Third, since the peak is so rigidly defined, the need for Cerenkov identification of kaons is eliminated. Using the Cerenkov analysis would put an upper limit of $\sim 1 \emptyset \emptyset \mathrm{GeV}$ on the energy for analyzable events (see figure 2.13). By not relying on the Cerenkov analysis we are able to fit the $\phi$ peak out to $\sim 165 \mathrm{GeV}$ where the geometric acceptance approaches zero.

Section 1: Calorimeter Acceptance for the $\phi$

The method for measuring the calorimeter acceptance has been discussed in chapters 4 and 7 . For the $\phi$ acceptance, a sample of events within $+/-7 \mathrm{MeV}$ of the $\phi$ mass was chosen. If either track was a well identified pion in the cerenkov counters, the event was cut. The charged track energy was required to be within $35<E_{\text {TOT }}<165 \mathrm{GeV}$. The PAD distributions were measured in three different regions of $E_{H C}=\left(E_{\text {TOT }}-E_{L G}\right) ; 35-55,55-75$ and $75-150 \mathrm{GeV}$. 
Due to the much smaller sample of $\varnothing$ data, the acceptance function could not be measured as well for the $\phi$ as for the $\rho$. Figure 8.2 shows the three $\phi$ measurements plotted with the acceptance curve measured for the $\rho$. The measurements are totally consistent with the measurement from the $\rho$ data.

In chapter 7 it was shown that the calorimeter acceptance, with a $5^{\prime \prime}$ fiducial cut at the $\mathrm{HC}$, was dominated by the energy seen by the $\mathrm{HC}$ and not by geometric effects. Because the geometrical differences are not important and because of the consistency of the $\phi$ measurement with the $\rho$ measurement, the HC acceptance curve determined from the $\rho$ data was used for the $\phi$ analysis.

Section 2: The Energy Dependence of the $\phi$ cross Section

The number of observed events can be expressed in terms of the cross section as:

$N=N_{\gamma} \cdot L T \cdot n t \cdot A C C \cdot \epsilon_{D E C A Y}(E) \cdot \epsilon_{M T} \quad \cdot B R \cdot \prod_{i} \epsilon_{i} \cdot \sigma(\gamma+P \rightarrow \phi+P)$ where

$$
\begin{aligned}
\mathrm{BR} & =\varnothing \text { branching ratio into } \mathrm{K}^{+} \mathrm{K}^{-}=.486 \\
\epsilon_{O E C A Y}^{(E)} & =\text { correction for kaon decay in flight }
\end{aligned}
$$

All other quantities are as described in chapter 7 , and in 


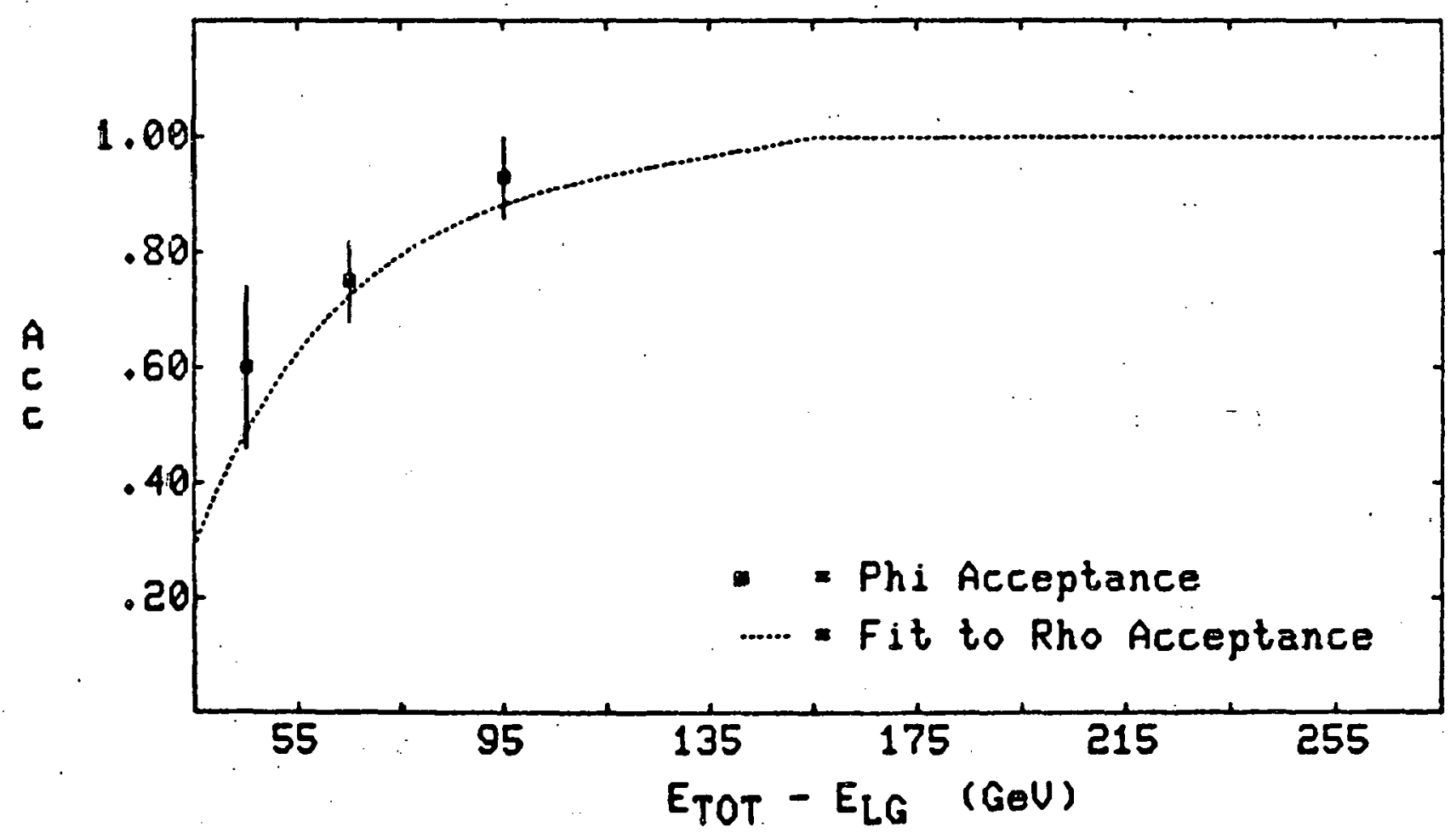


appendix D. The expression for the cross-section is thus:

$$
\sigma(\gamma+\rho \rightarrow \phi+\rho)=\frac{1.848 \times 10^{6}}{A_{C C}(E) \cdot \epsilon_{D E C A Y}^{(E)}} \times \frac{N_{\phi}}{N_{\gamma}} \mu b \quad(8.2)
$$

The $\phi$ yield, $\mathrm{N}_{\phi} / \mathrm{Acc}$, was obtained by integrating equation 8.1, excluding $B G$, over the $\mathrm{K}^{+} \mathrm{K}^{-}$spectrum in each energy region and dividing by the acceptance determined from the Monte carlo. There were no problems with the acceptance variation as a function of mass over the $\phi$ peak as there were with the broad $\rho$ peak.

Figures 8.3 to 8.5 show the $\phi$ signal in the 9 . different energy regions analyzed. Table 8.1 summarizes the cross sections obtained with equation 8.2 and the yields from the fits using equation 8.1 . Table 8.2 lists the mass resolution that was used in the fit for each energy region as determined from the Monte Carlo. The errors are the statistical errors from the fit only. Figure 8.6 shows a plot of the $\phi$ cross section as a function of energy.

The measurement of the $\phi$ cross section as a function of energy is consistent with a rise in the $\phi$ cross section from $\emptyset .60$ ub at low energy. ( $45 \mathrm{GeV}$ ) to $\emptyset .73$ ub at high energy ( 140 GeV). With the systematic uncertainties in the calorimeter 


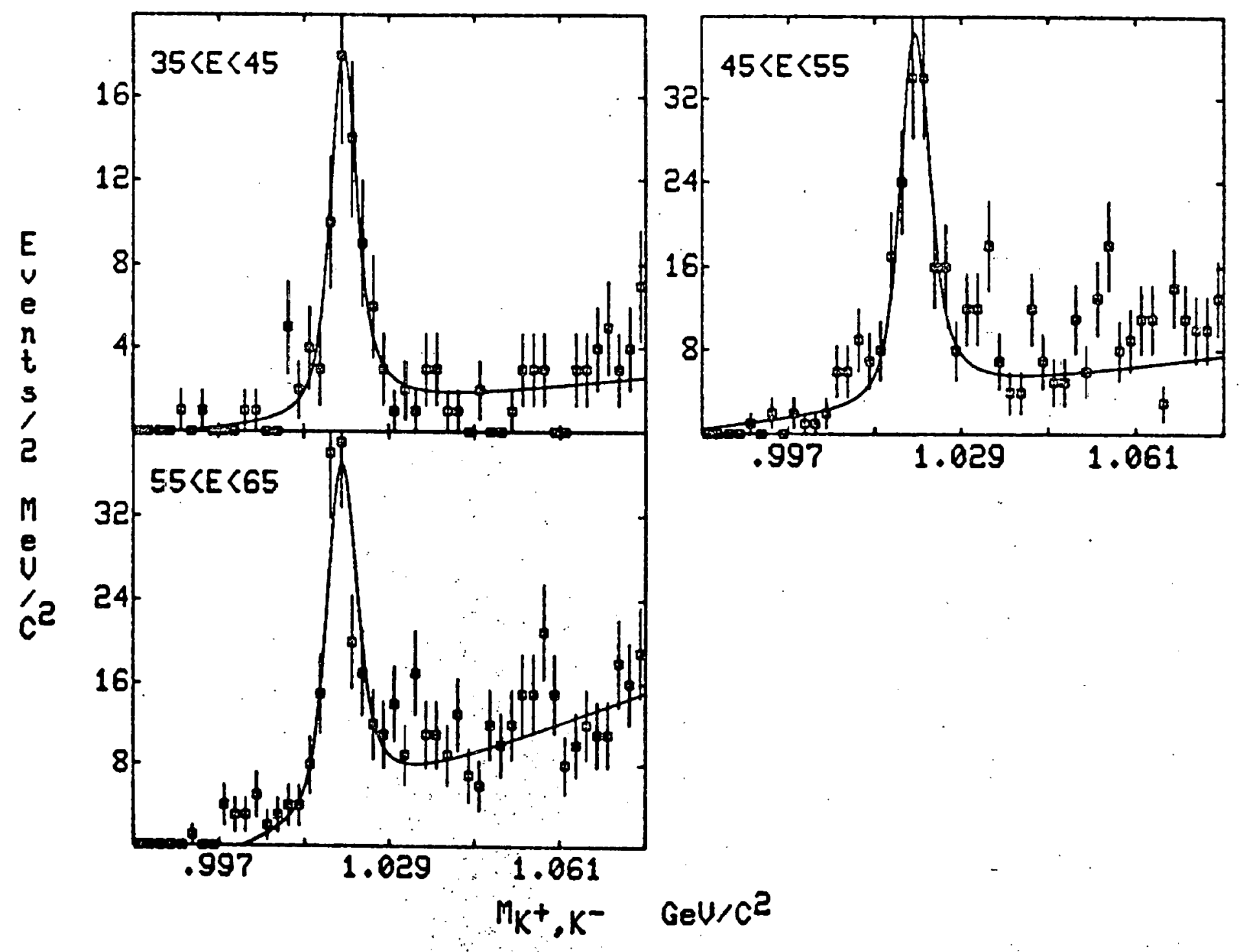

Figure 8.3 


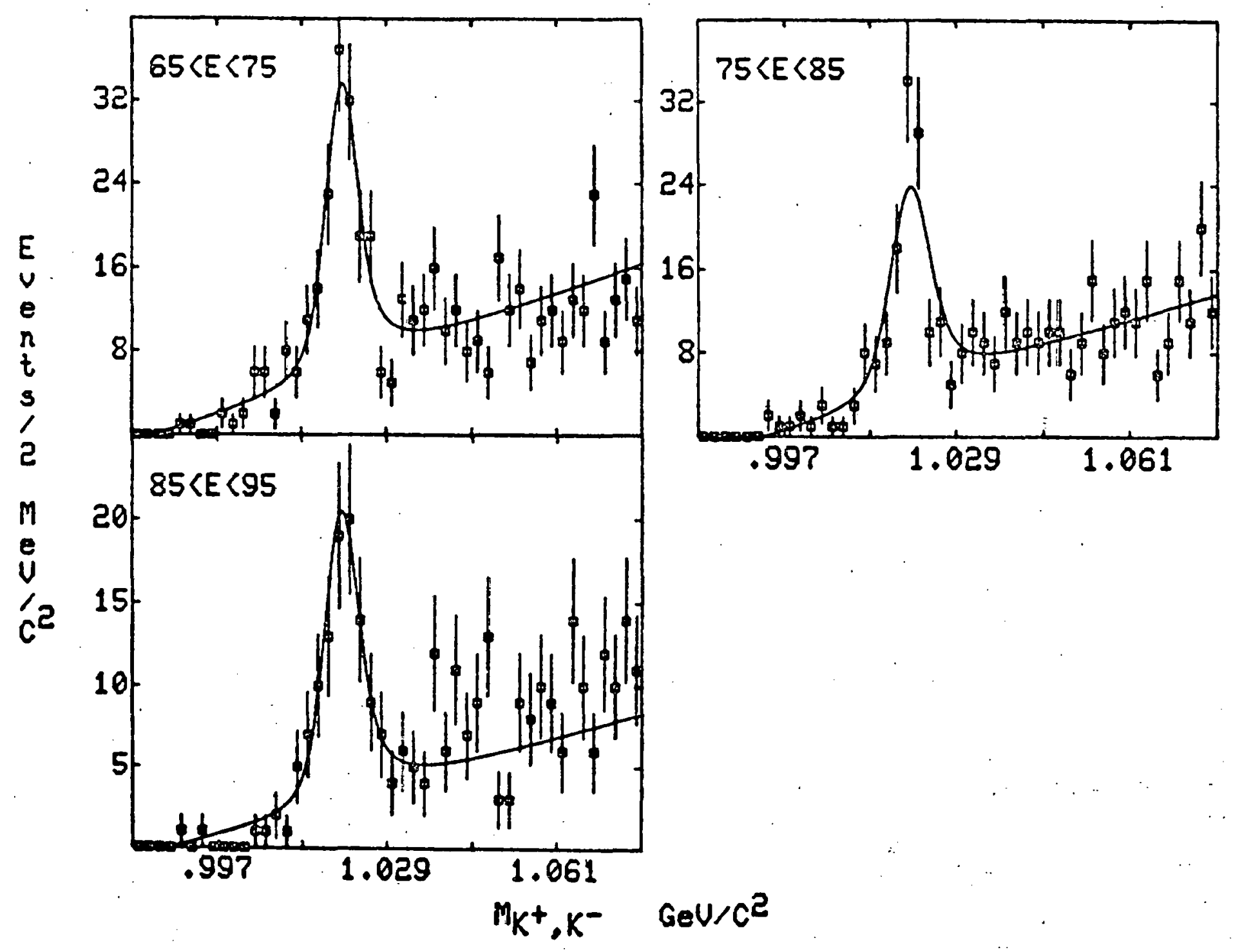

Figure 8.4 


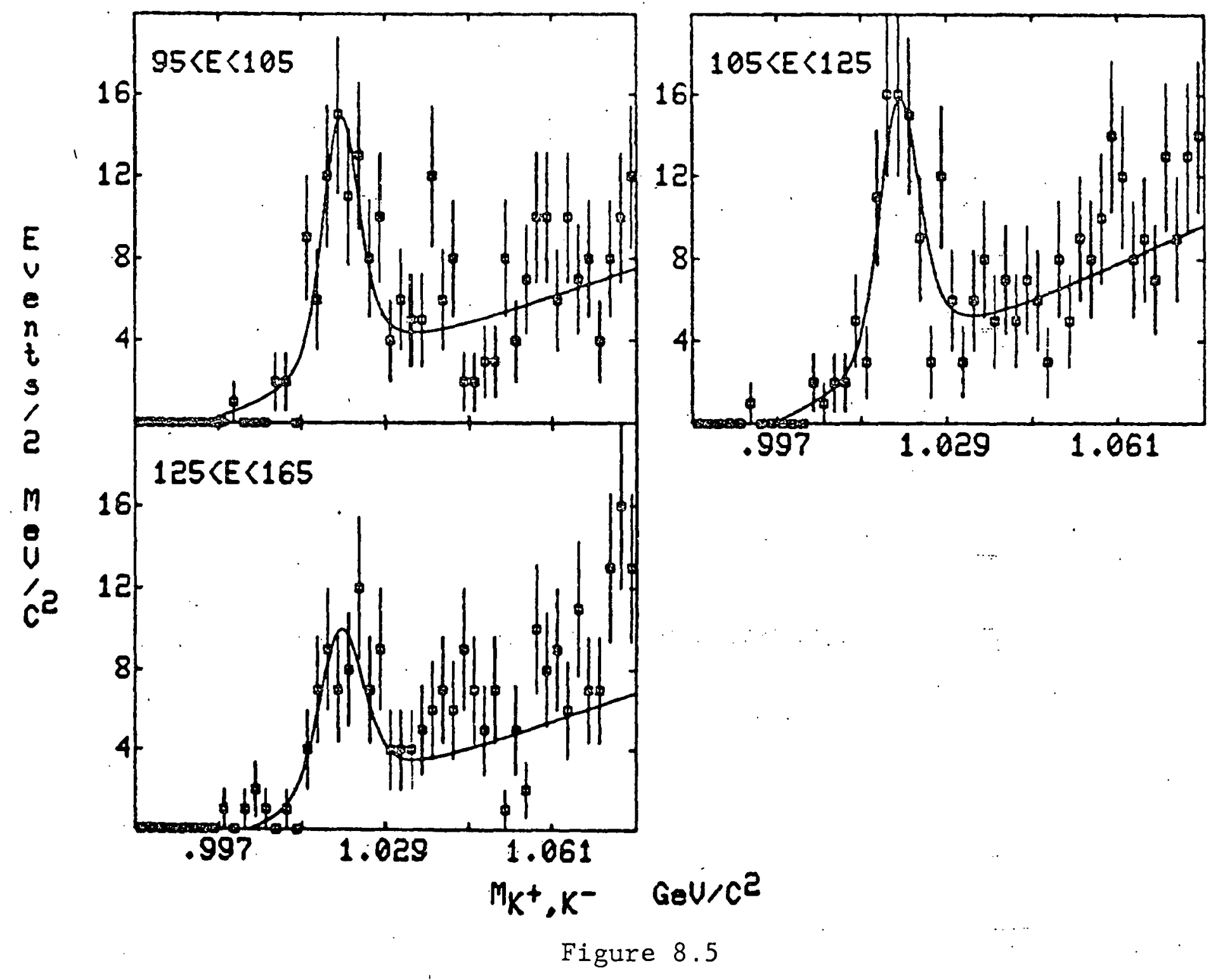


Table 8.1

\begin{tabular}{|c|c|c|c|c|c|c|}
\hline$E(\mathrm{GeV})$ & $\underline{\mathrm{N}}_{\phi}$ & $\underline{N}_{\gamma}\left(x \perp 0^{q}\right)$ & $\operatorname{Acc}(E)$ & $\epsilon_{D E C A Y} \frac{(E)}{1}$ & $\sigma(E) \mu b$ & $\Delta \sigma \mu b$ \\
\hline $35-45$ & 62.9 & 3.256 & .0619 & .916 & .630 & .079 \\
\hline $45-55$ & 137.3 & 2.632 & .1790 & .932 & .578 & .049 \\
\hline $55-65$ & 136.2 & 2.139 & .2130 & .943 & .586 & .050 \\
\hline $65-75$ & 118.1 & 1.740 & .2120 & .951 & .622 & .057 \\
\hline $75-85$ & 96.7 & 1.416 & .1990 & .957 & .663 & .067 \\
\hline $85-95$ & 79.8 & 1.153 & .1830 & .962 & .726 & .081 \\
\hline $95-105$ & 59.4 & .9334 & .1570 & .965 & .776 & .100 \\
\hline $105-125$ & $6 \dot{4.9}$ & 1.335 & .1320 & .970 & .702 & .087 \\
\hline $125-165$ & 46.7 & 1.380 & .0845 & .975 & .759 & .110 \\
\hline
\end{tabular}

Table. 8.2

E (GeV) Mass Resolution (MeV)

$\begin{array}{rl}35-45 & 1.40 \\ 45-55 & 1.69 \\ 55-65 & 1.89 \\ 65-75 & 2.10 \\ 75-85 & 2.28 \\ 85-95 & 2.37 \\ 95-105 & 2.58 \\ 105-125 & 2.81 \\ 125-165 & 3.42\end{array}$




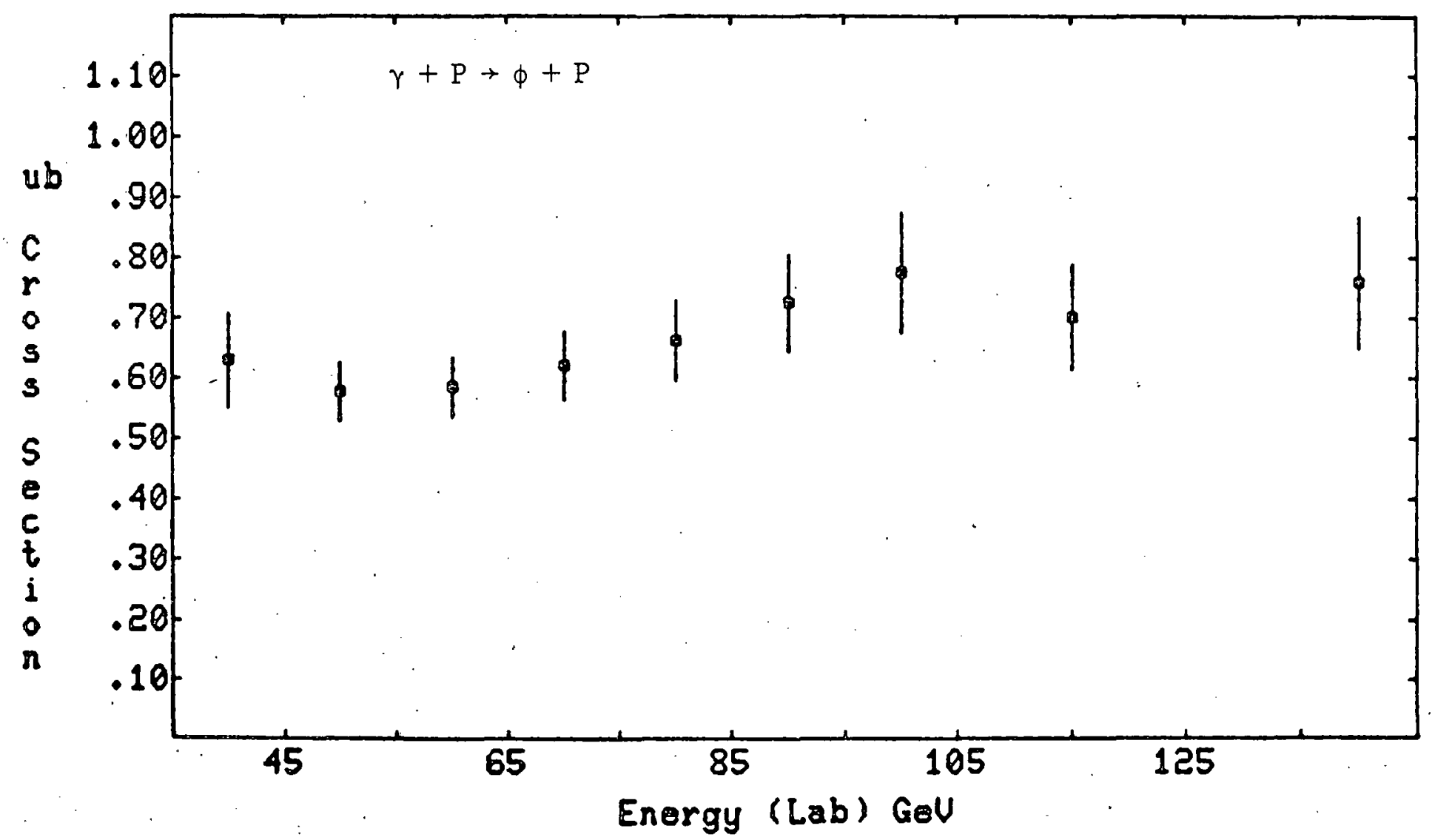


acceptance, this measurement is also consistent with a flat cross section of $0.61<\sigma<0.67$ ub over the entire energy region.

Section 3: $t$ Dependence for Elastic $\phi$ Photoproduction

The four-momentum squared transfer was computed for each event using equation 5.1. The yield in any given $t$ interval was determined by fitting the 2-kaon mass distribution in that interval with equation 8.1. Again, all the parameters were fixed except for the background polynomial and the overall normalization. The mass resolution parameter, $\sigma$, was determined from the Monte Carlo for each $t$ interval integrated over all energies. There were insufficient data to study the $t$ distribution in different energy regions.

The number of observed events can be related: to the differential cross section as:

$\mathrm{N}=\mathrm{N}_{\gamma} \cdot \mathrm{LT} \cdot \mathrm{nt} \cdot \mathrm{ACC} \cdot \epsilon_{O E C A Y}(E) \cdot \epsilon_{M T}(t) \cdot \mathrm{BR} \cdot \mathrm{BW} \cdot \prod_{i} \epsilon_{i} \cdot \frac{d \sigma}{d t}(\gamma+\mathrm{P} \rightarrow \psi+P)$

where all the terms are as described previously. For the differential cross section we obtain:

$\frac{d \sigma}{d t}(\gamma+\rho \rightarrow \phi+\rho)=\frac{9.101 \times 10^{5}}{B W} \times \frac{\epsilon_{M T}^{-1}(t)}{\epsilon_{D E C A Y}(E)} \times \frac{N_{\phi}}{N_{\gamma}} \frac{\mu i b}{(G V / C)^{2}}$ 
Figures 8.7 to 8.8 show the fits to the $\phi$ signal in each $t$ interval. Table 8.3 summarizes the cross sections obtained with equation 8.1 and yields from the fits. Table 8.4 lists the mass resolution that was used in the fit for each $t$ region as determined from the Monte Carlo. Figure 8.9 shows a plot of $d \sigma / d t$ verses $t$. The fit is an exponential with a quadratic term:

$$
\left.\frac{d \sigma}{d t}\right|_{t=0} \times e^{-b t+c t^{2}}
$$

where

$$
\begin{aligned}
d \sigma /\left.d t\right|_{t=0} & =4.06+/-.27 \mu b /(\mathrm{Gev} / \mathrm{c})^{2} \\
b & =7.53+/-.53(\mathrm{GeV} / \mathrm{c})^{-2} \\
c & =2.50+/-.73(\mathrm{GeV} / \mathrm{c})^{-4}
\end{aligned}
$$

For $t<.4 \mathrm{GeV}^{2} / \mathrm{c}^{2}$, the $t$ distribution can be represented by an exponential function with a sinqle slope parameter:

$$
b=6.74+/-.4(\mathrm{GeV} / \mathrm{c})^{-2}
$$

Section 4: Measurement of $\mathrm{d} \sigma /\left.\mathrm{d} t\right|_{t=0}$

If we assume that the shape of the $t$ distribution does not change appreciably with energy then the shape of the $t$ distribution measured in section 4 can be used with the cross sections of section 3 to compute $d \sigma /\left.d t\right|_{t=0}$ in different energy regions. 


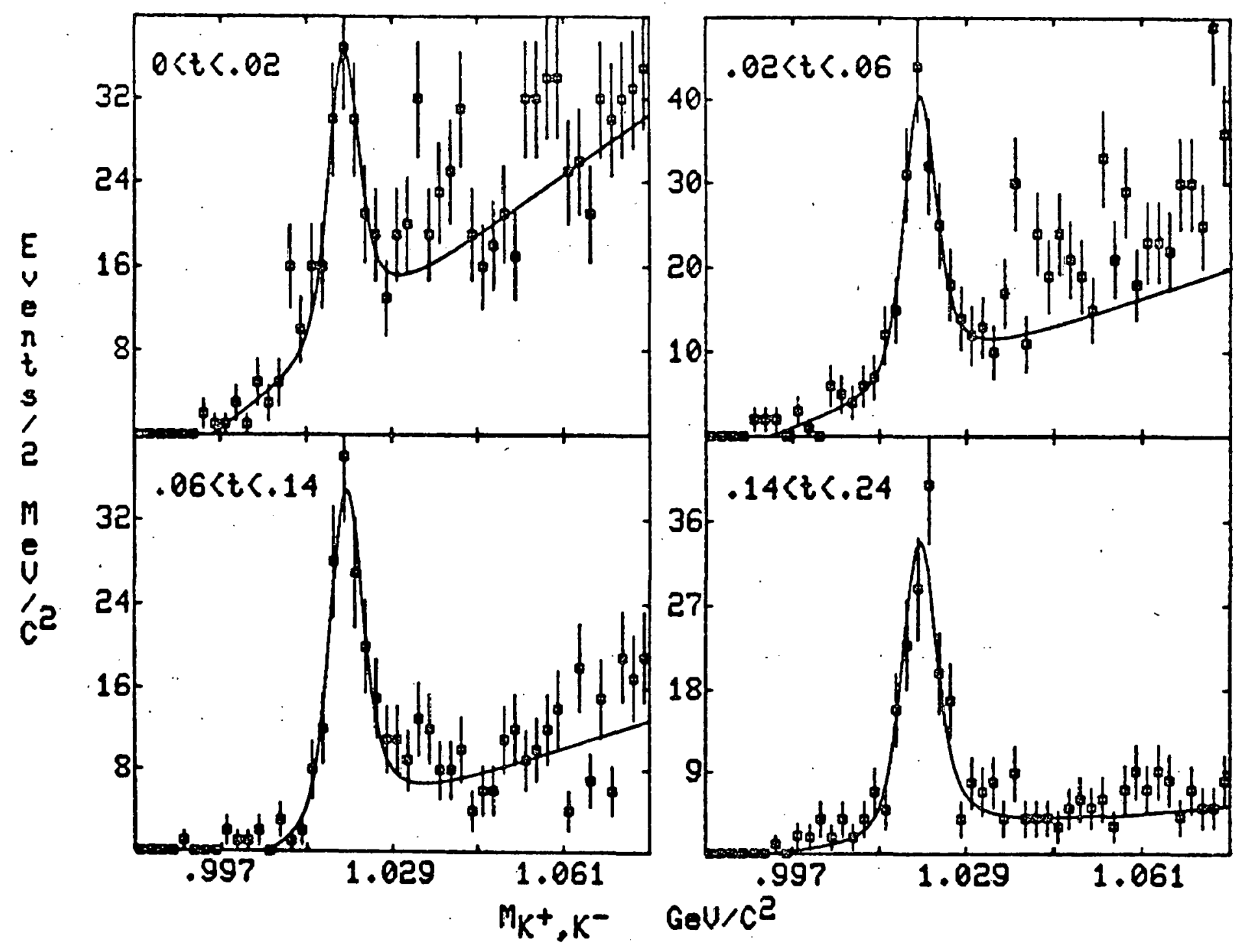

음

Figure 8.7 


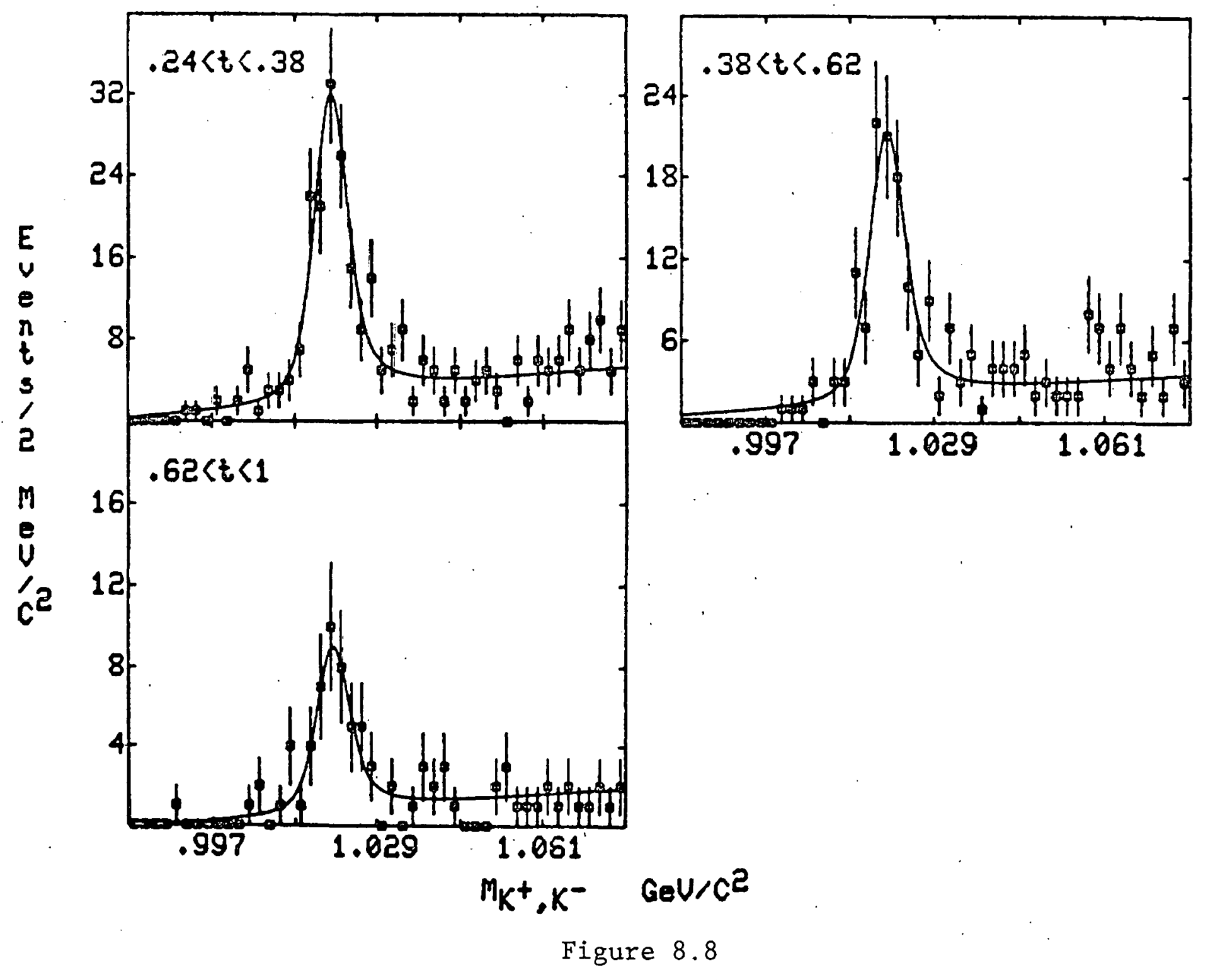


Table 8.3

\begin{tabular}{|c|c|c|c|c|c|}
\hline$t \underline{\mathrm{GeV}}{ }^{2} \angle \mathrm{c}^{2}$ & $\underline{N}_{\phi}$ & $\underline{A C C}(t)$ & $\epsilon_{M T}^{-1}(t)$ & $\mathrm{d} \sigma \angle \mathrm{dt}{ }^{*}$ & $\Delta\left(d \sigma\langle d t)^{*}\right.$ \\
\hline$\emptyset-.02$ & 111.6 & .169 & .908 & 3.62 & .34 \\
\hline $.02-.06$ & 140.5 & .119 & .965 & 3.43 & .29 \\
\hline $.06-.14$ & 142.1 & .110 & .983 & 1.91 & .16 \\
\hline $.14-.24$ & 141.2 & .167 & .983 & 1.00 & .084 \\
\hline $.24-.38$ & 131.8 & .202 & .983 & .553 & .048 \\
\hline $.38-.62$ & 88.9 & .230 & .983 & .191 & .020 \\
\hline $.62-1.0$ & 37.3 & $.23 \emptyset$ & .983 & .0506 & .0083 \\
\hline
\end{tabular}

* ub/(GeV/c $)^{2}$ 
Table 8.4

$\begin{array}{lc}\underline{t}\left(\mathrm{GeV}^{2} / \mathrm{c}^{2}\right) & \text { Mass Resolution(MeV) } \\ 0-.02 & 1.85 \\ .02-.06 & 1.96 \\ .06-.14 & 2.19 \\ .14-.24 & 2.22 \\ .24-.38 & 2.26 \\ .38-.62 & 2.36 \\ .62-1.0 & 2.30\end{array}$




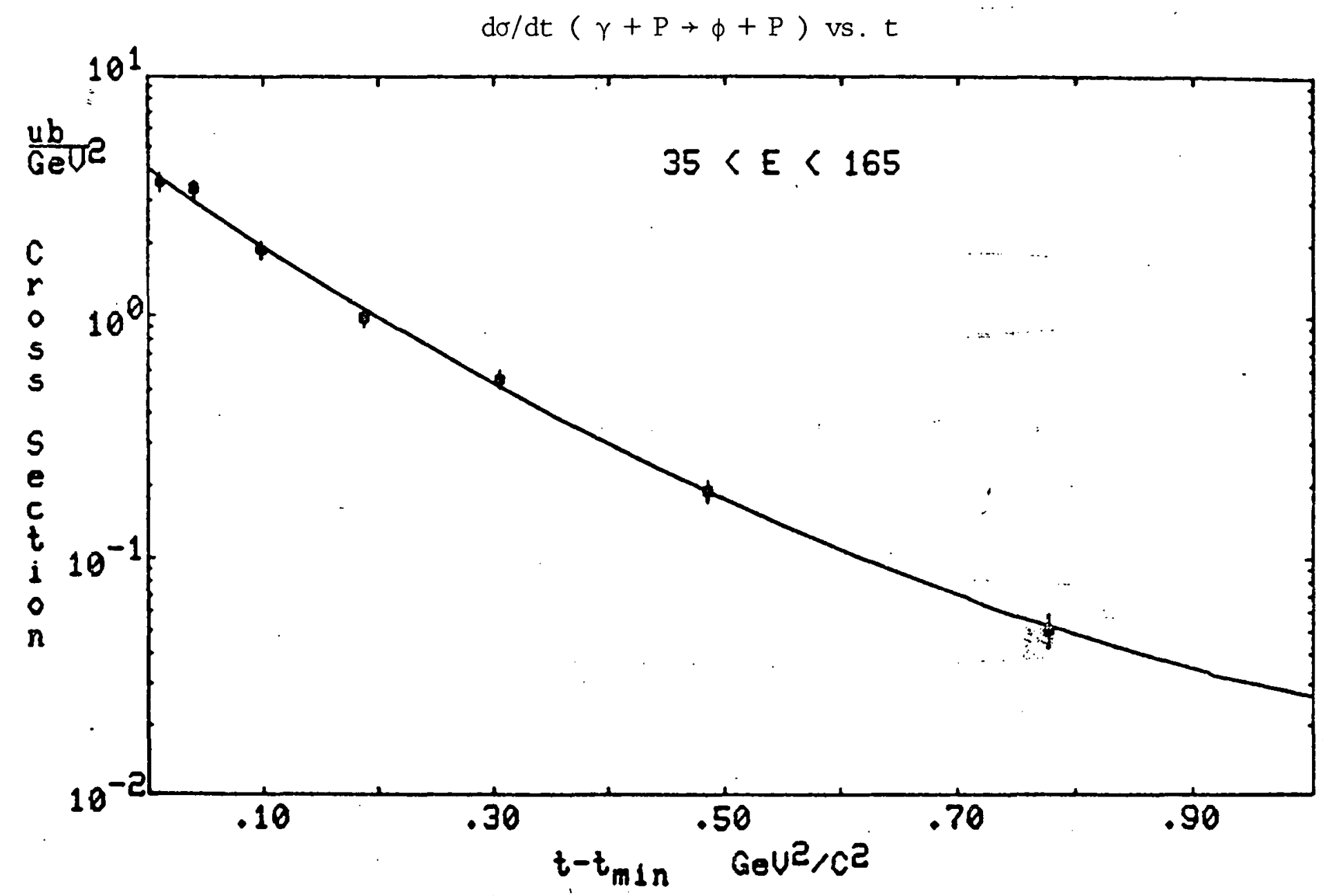

Figure 8.9 


$$
\left.\frac{d \sigma}{d t}\right|_{t=0}=\sigma(E) / \int e^{-b t+c t^{2}} d t
$$

Using the values for $b$ and $c$ from the previous section, we obtain:

$$
\int_{0}^{1} e^{-7.53 t+2.50 t^{2}} d t=.148
$$

Dividing the cross sections from section 3 by the value of the above integral obtains the results listed in table 8.5 for $d \sigma /\left.d t\right|_{t=0}$ as a function of energy.

Table 8.5

$\begin{array}{rl}\underline{E}(\mathrm{GeV}) & \left.\underline{\mathrm{d} \sigma} \angle \mathrm{dt}\right|_{t=0} \mathrm{ub} /\left(\mathrm{GeV}^{2} / \mathrm{c}^{2}:\right) \\ 35-45 & 4.25+/-.53 \\ 45-55 & 3.90+/-.33 \\ 55-65 & 3.95+/-.34 \\ 65-75 & 4.20+/-.38 \\ 75-85 & 4.47+/-.45 \\ 85-95 & 4.90+/-.55 \\ 95-105 & 5.24+/-.67 \\ 105-125 & 4.74+/-.59 \\ 125-165 & 5.12+/-.74\end{array}$


Section 5: Diffractive Inelastic $\phi$ Photoproduction

The diffractive inelastic $\phi$ cross section has been measured for incident photon energies between $35<E<165 \mathrm{GeV}$ and $\emptyset<t<1 \mathrm{GeV}^{2} / \mathrm{c}^{2}$. Inelastic events were those that were inconsistent with elastic... production as determined by the analysis of the recoil detector.

As discussed in chapter $6,30 \%$ of the $\phi$ signal was $k_{L}^{0}$ induced. Further, all of the $k_{L}^{0}$ background was inelastic. Although the observation that the $K_{L}^{0}$ induced background was inelastic eliminated the problem of making a $K_{L}^{0}$ subtraction for the measurement of the $s$ and $t$ dependence of elastic $\phi$ photoproduction, it necessitated making a large subtraction to measure the diffractive inelastic cross section. There were insufficient $k_{L}^{0}$ data to make this subtraction in different $t$ bins, therefore the diffractive inelastic cross : section integrated over $t$ was measured. Using the function representing the fraction of diffractive events that are inelastic as a function of $t$ measured from the $\rho$ analysis, $f(t)$ (eq. 7.16), and $d \sigma / d t$ for elastic production neasured from the $\phi$ analysis, a prediction for the diffractive inelastic $\phi$ cross section was also made. This prediction agrees extremely well with the measured diffractive inelastic $\phi$ cross section. 
Pin 5 events with a di-kaon mass in the $\varnothing$ mass region were used from the $K_{L}^{0}$ runs and the photon runs. A tight target cut was made $(-262.5 "<z<-255.5 ")$ to ensure acceptance in the RC fiducial volume and eliminate any contamination from the target end caps. It was required that at least one track be identified as a kaon by the Cerenkov counter system to enhance the signal to noise ratio. The cerenkov requirement was necessary for measuring the inelastic $\phi$ peak in the $k_{L}^{\circ}$ data. A Monte Carlo was analyzed to determine the mass resolution and the the fraction of elastic events that were misidentrfied as inelastic by $\mathrm{RC} 6$. Three $\mathrm{K}^{+} \mathrm{K}^{-}$. invariant mass distributions, inelastic photoproduced, inelastic $k_{L}^{\circ}$ produced and elastic photoproduced, were fit with equation 8.1 with all parameters fixed except the normalization and the background shape. Figure 8.10 shows the fits to these mass plots.

The ratio of the diffractive inelastic cross section to the total diffractive cross section is:

$$
\frac{\sigma_{\text {INEL }}}{\sigma_{\text {Tot }}}=\frac{N_{i}^{\top}}{N_{i}^{\top}+N_{e}^{\top}}
$$

where $\mathrm{N}_{i}^{\top}=$ \#true inelastic diffractive events

$$
\mathrm{N}_{e}^{\top}=\# \text { true elastic diffractive events }
$$

and all other common normalization factors cancel. 


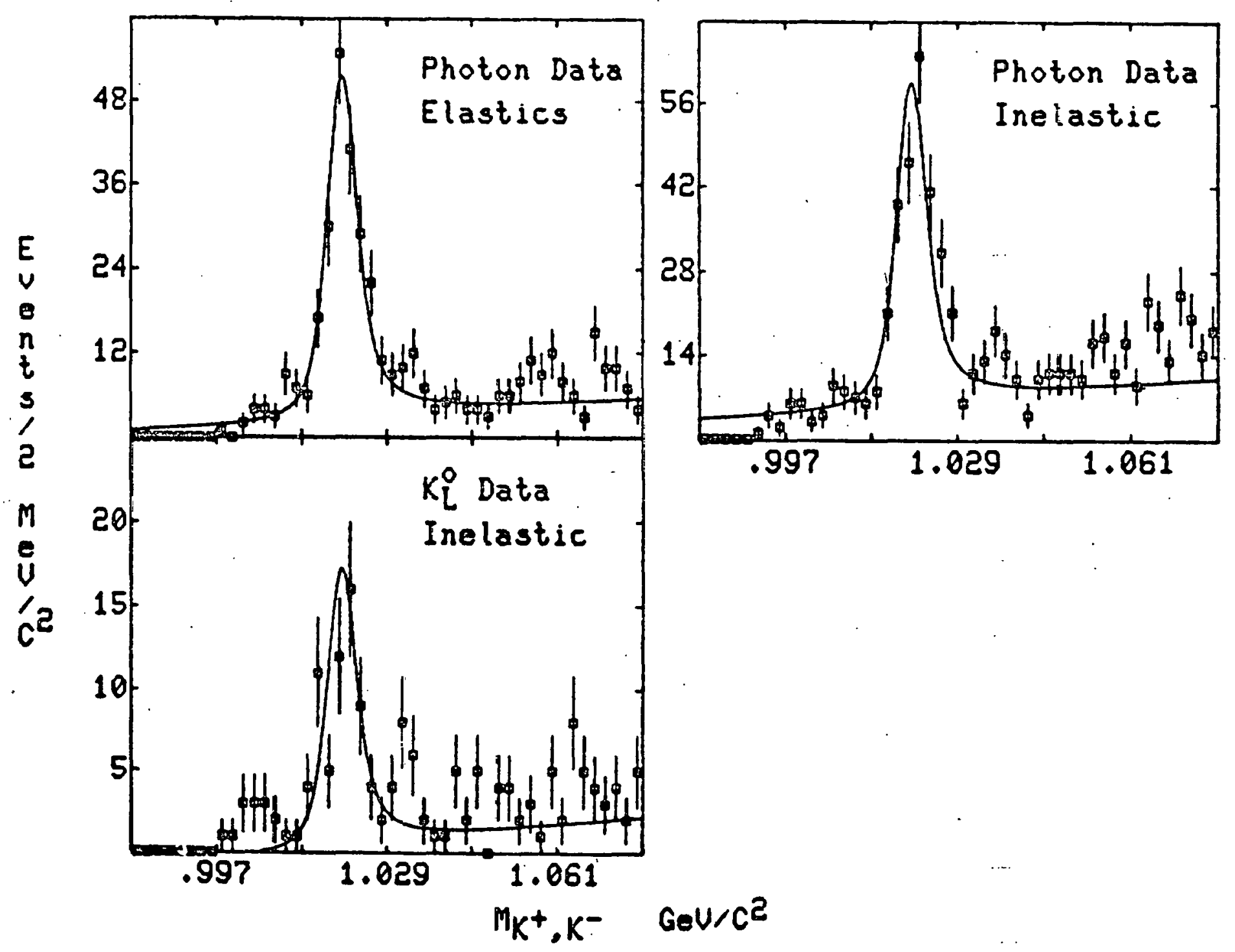

Figure 8.10 
$\mathrm{N}_{i}^{\top}$ must be corrected for $\mathrm{K}_{L}^{\sigma}$ contamination and elastic events misidentıfied as inelastic by RC6.

$$
N_{i}^{\top}=N_{i}^{0}-f \cdot N_{K}-\frac{\varepsilon}{(1-\varepsilon)} N_{e}^{0}
$$

where

$$
\begin{aligned}
& \mathrm{N}_{i}^{\top}=\text { \#true inelastic } \phi \text { 's } \\
& \mathrm{f} \quad=2.234: \mathrm{K}_{L}^{o} \text { scale factor (appendix E) } \\
& \mathrm{N}_{K}=67+/-8: \# \phi^{\prime} \mathrm{s} \text { from } \mathrm{K}_{L}^{0} \text { runs (figure } 8.1 \emptyset \text { ) } \\
& \varepsilon=.055 \text { : fraction of elastıc events misidenti- } \\
& \text { fied as inelastic by RC6 for } \emptyset<t<1 \quad \mathrm{GeV}^{2} / \mathrm{c}^{2} \\
& \mathrm{~N}_{i}^{0}=212+/-14: \text { \# observed inelastic } \phi \text { 's } \\
& \text { (figure 8.10) }
\end{aligned}
$$

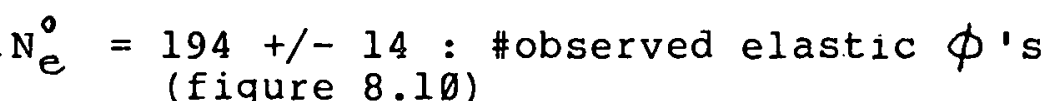

Substituting the above values into equation 8.5 we obtain:

$$
N_{i}^{\top}=51+/-23
$$

For the true number of elastic events we obtain:

$$
N_{e}^{\top}=\frac{N_{e}^{0}}{(1-\varepsilon)}=205 \pm 15
$$

The large error in $\mathrm{N}_{i}^{\top}$ is a result of the $\mathrm{K}_{L}^{0}$ subtraction. 
176

Substituting equations 8.6 and 8.7 into equation 8.4 we obtain for the ratio of the diffractive inelastic cross section to the total diffractive cross section:

$$
\frac{\sigma_{\text {INEL }}}{\sigma_{\text {TOT }}}=\frac{51}{205+51}=0.20 \pm .09(8.8)
$$

If it is assumed that the fraction of diffractive events that are inelastic as a function of $t$ is the same for all vector mesons, then $\sigma_{\text {INCL }} / \sigma_{\text {TOT }}$ for the $\phi$ can be computed using $f(t)$ from the $e$ analysis (eq. 7.16) and $d \sigma / d t$ for elastic production from the $\phi$ analysis:

$$
\frac{d \sigma}{d t_{\text {INELASTIC }}}(\gamma+P \rightarrow \varnothing+P)=\frac{f(t)}{1-f(t)} \times \frac{d \sigma}{d t_{\text {ELASTIC }}}(\gamma+P \rightarrow \varnothing+P)
$$

$$
\begin{aligned}
& \frac{d \sigma}{d t_{\text {TOTAL }}}(\gamma+P \rightarrow \varnothing+P)=\frac{d \sigma}{d t_{\text {iNELASTIC }}}(\gamma+P \rightarrow \varnothing+P)+\frac{d \sigma}{d t_{\text {ELASTIC }}}(\gamma \rightarrow P \rightarrow \phi) \\
& =\frac{1}{1-f(t)} \times \frac{d \sigma}{d t_{\text {EUSTC }}}(\gamma+P \rightarrow \varnothing+P)
\end{aligned}
$$


Integrating over $t$, we obtain:

$$
\frac{\sigma_{\text {INEL }}}{\sigma_{\text {TOT }}}=\frac{\int \frac{f(t)}{1-f(t)} \times \frac{d \sigma}{d t_{\text {ELAS }}} d t}{\int \frac{1}{1-f(t)} \times \frac{d \sigma}{d t_{\text {ELAS }}} d t} .
$$

using the results from section 3 :

$$
\frac{d \sigma}{d t_{\text {ELASTIC }}}(\gamma+P \rightarrow \varnothing+P)=4.06 e^{-7.53 t+2.50 t^{2}} \frac{\mu b}{(\mathrm{GeV} / \mathrm{C})^{2}}
$$

and the result for $f(t)$ from chapter 7 , section 6 :

$$
f(t)=\left[1+8.0905 e^{-8.22 t+2.3 t^{2}+4.0 t}\right]^{-1} \text {. }
$$

we substitute for $f(t)$ and $d \sigma / d t_{\text {Elastic }}$ in equation 8.9 and obtain;

$$
\frac{\sigma_{\text {INEL }}}{\sigma_{\text {TOT }}}=.20
$$

The agreement between equations 8.10 and 8.8 is excellent. 
Chapter IX

$\psi$ Analysis

The energy and $t$ dependence for diffractive $\psi$ photoproduction between 60 and $300 \mathrm{GeV}$ were studied. A total of 1650 2-track events on deuterium and 1752 -track events on hydrogen were recorded under $\mathrm{e}^{+} \mathrm{e}^{-}$and $\mu^{+} \mu^{-}$triggers. The majority of this work has been published already and the reader is referred to [12] for a detailed discussion. A brief summary of the published results is given here along with a new analysis of diffractive inelastic $\psi$ photoproduction.

Section 1: Energy Dependence of $\psi$ Photoproduction

The energy dependence for diffractive $\psi$ photoproduction on liquid hydrogen and liquid deuterium is shown in figure 9.1. The cross section rises significantly $(\sim x 3)$ from 60 to $3 \emptyset 0 \mathrm{GeV}$. Assuming a linear dependence on energy, a slope of $.007+/-.0014 \mathrm{nb} / \mathrm{GeV}$ describes this rise. This behavior is markedly different from the measurements presented in chapters 7 and 8 on the $\rho$ and $\phi$ cross sections, which are independent of energy, or perhaps rising approximately 108 , with increasing energy.

Section 2: $t$ Dependence for Elastic $\psi$ Photoproduction

The recoil analysis described in chapter 5 was pertormed on the liquid hydrogen $\psi$ data. Figure 9.2 shows the elastic differential cross section as a function of $t$. 


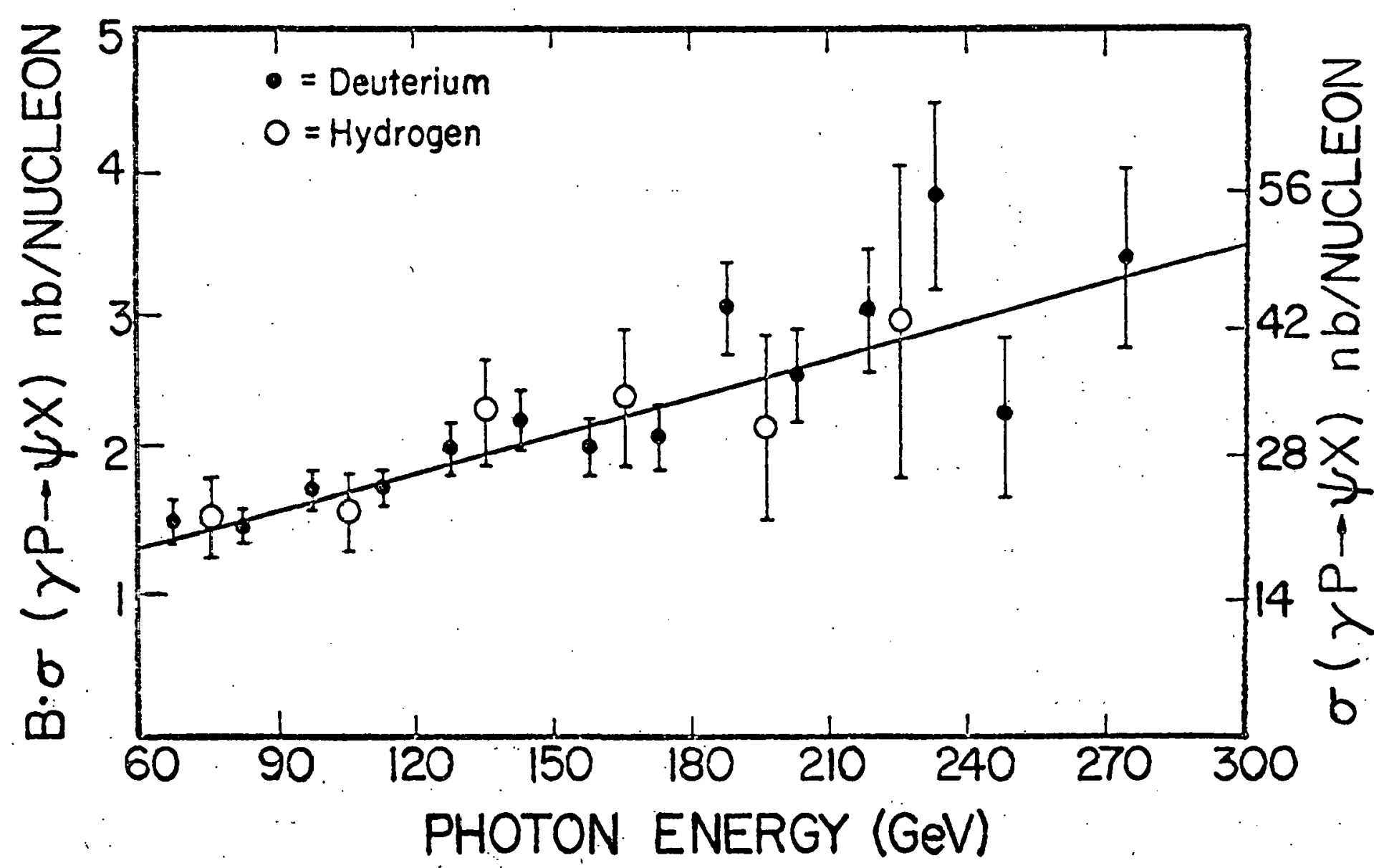


The curve is a fit to an exponential function with a quadratic term.

$$
\frac{d \sigma}{d t}=A_{0} e^{-b t+c t^{2}}
$$

with

$$
\begin{aligned}
& A=79.8+/-13.3 \mathrm{nb} /(\mathrm{GeV} / \mathrm{c})^{2} \\
& \mathrm{~b}=5.55+/-1.18(\mathrm{GeV} / \mathrm{c})^{-2} \\
& \mathrm{c}=2.85+/-1.32(\mathrm{GeV} / \mathrm{c})^{-4}
\end{aligned}
$$

As described in reference [12], the slope of the elastic differential cross section on hydrogen was used to extrapolate under the coherent peak in the deuterium differential cross section from $t=.1$ to $t=0$. Figure 9.3 shows the results for $d \sigma /\left.d t\right|_{t=0}$ from the deuterium data in four difterent energy regions obtained by extrapolation with the elastic differential cross section measured on hydrogen.

Section 3: Diffractive Inelastic $\psi$ Production

The fraction of diffractive $\psi$ photoproduction that is inelactic : was measured on hydrogen from $t=\emptyset$ to $t=1 . \emptyset 4 \mathrm{GeV}^{2} / \mathrm{c}^{2}$. Inelastic events were those that were inconsistent with elastic production as determined by the analysis of the recoil detector. Events with a di-muon or di-electron mass in the range $2.85 \mathrm{GeV} / \mathrm{c}^{2}$ to $3.35 \mathrm{GeV} / \mathrm{c}^{2}$ were used. At small $t$ the data were corrected for high mass 


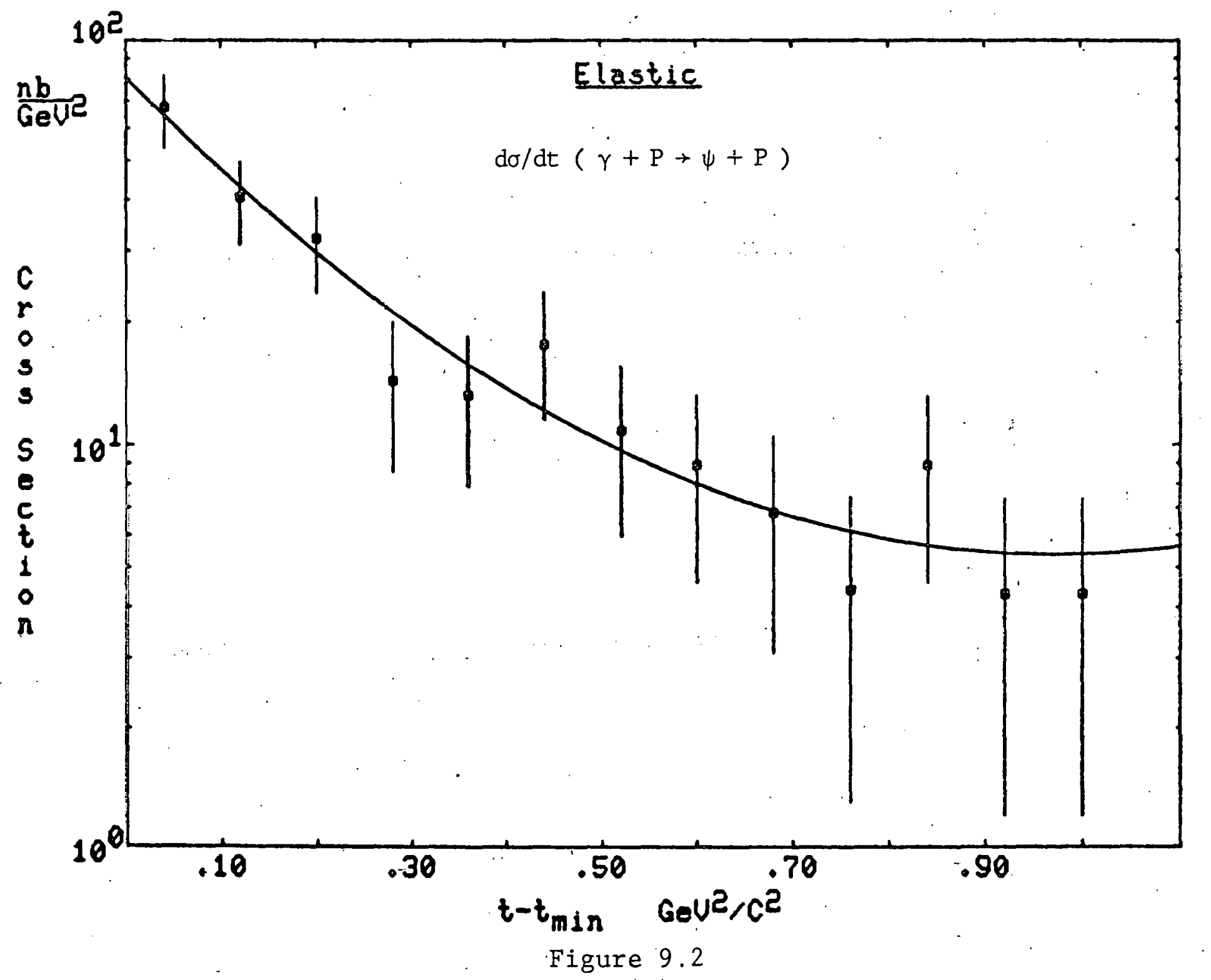

$\stackrel{\infty}{\bullet}$ 


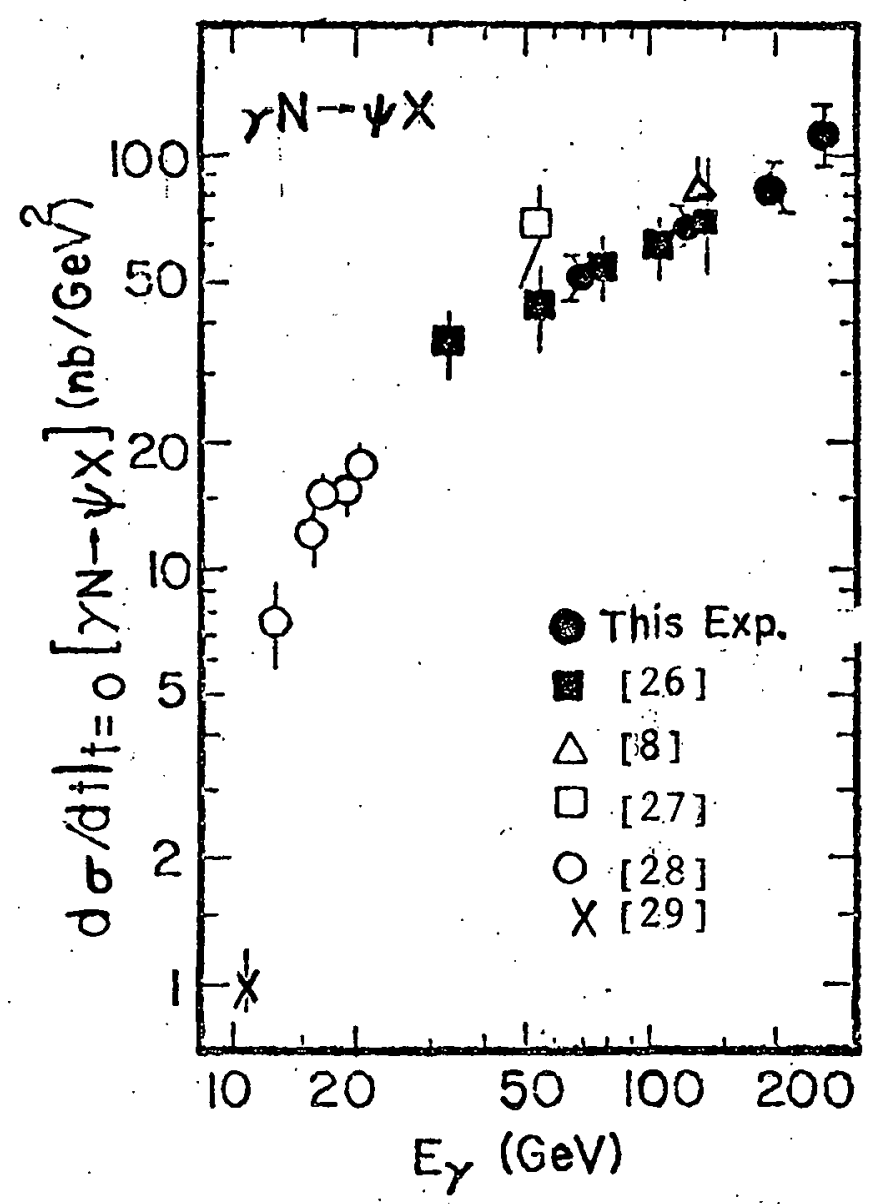

Figure 9.3 
Bethe-Heitler pair production, which is strongly peaked near $t=\emptyset \mathrm{GeV}^{2} / \mathrm{c}^{2}$. A Monte Carlo was analyzed to determine fraction of elastic events misidentified as inelastic by RC6. The true numbers of elastic and inelastic events are given by:

$$
\begin{aligned}
& N_{a}^{\top}=\left(N_{a}^{0}-N_{B H}\right) /(1-\epsilon) \\
& N_{i}^{\top}=N_{i}^{n}-\epsilon \cdot N_{a}^{\top}
\end{aligned}
$$

where

$$
\begin{aligned}
\mathrm{N}_{\mathrm{BH}} & =\text { Bethe-Heitler backround } \\
\epsilon & =\text { fraction misidentified by } \mathrm{RC} 6
\end{aligned}
$$

The ratio of the diffractive inelastic cross section to the total diffractive cross section as a function of $t$ is given by:

$$
\frac{\sigma_{\text {INEL }}(t)}{\sigma_{T O T}(t)}=\frac{N_{i}^{\top}(t)}{N_{i}^{\top}(t)+N_{e}^{\top}(t)}
$$

where other common normalization factors cancel. The results from this analysis are summarized in table 9.1. Figure 9.4 shows a plot of $\sigma_{\text {:NEL }}(t) / \sigma_{\text {ror }}(t)$ for the $\psi$. The curve is from equation 7.16, which is the fraction of diffractive $\rho$ production that is inelastic as a function of $t$ measured in chapter 7. The agreement is striking. 
Table 2.1

\begin{tabular}{rrrrrrr}
$\pm\left(\mathrm{GeV}^{2} / \mathrm{C}^{2}\right)$ & $\underline{\mathrm{N}}_{e}^{0}$ & \multicolumn{1}{c}{$\underline{\mathrm{N}}_{\mathrm{BH}}$} & $\underline{\underline{\mathrm{N}}}$ & $\underline{\sigma_{\text {weL }}} / \sigma_{\text {TOr }}+$ error \\
$0-.08$ & 41 & 11.57 & .031 & 3 & .064 & .037 \\
$.08-.16$ & 19 & 2.16 & .063 & 4 & .138 & .072 \\
$.16-.24$ & 15 & .76 & .032 & 5 & .236 & .095 \\
$.24-.4 \emptyset$ & 13 & .53 & .031 & .6 & .303 & .105 \\
$.40-.56$ & 13 & $\emptyset$ & .021 & 8 & .367 & .0105 \\
$.56-.72$ & 7 & $\emptyset$ & .02 & 7 & .49 & .13 \\
$.72-1.04$ & 10 & $\emptyset$ & .02 & 4 & .27 & .12
\end{tabular}




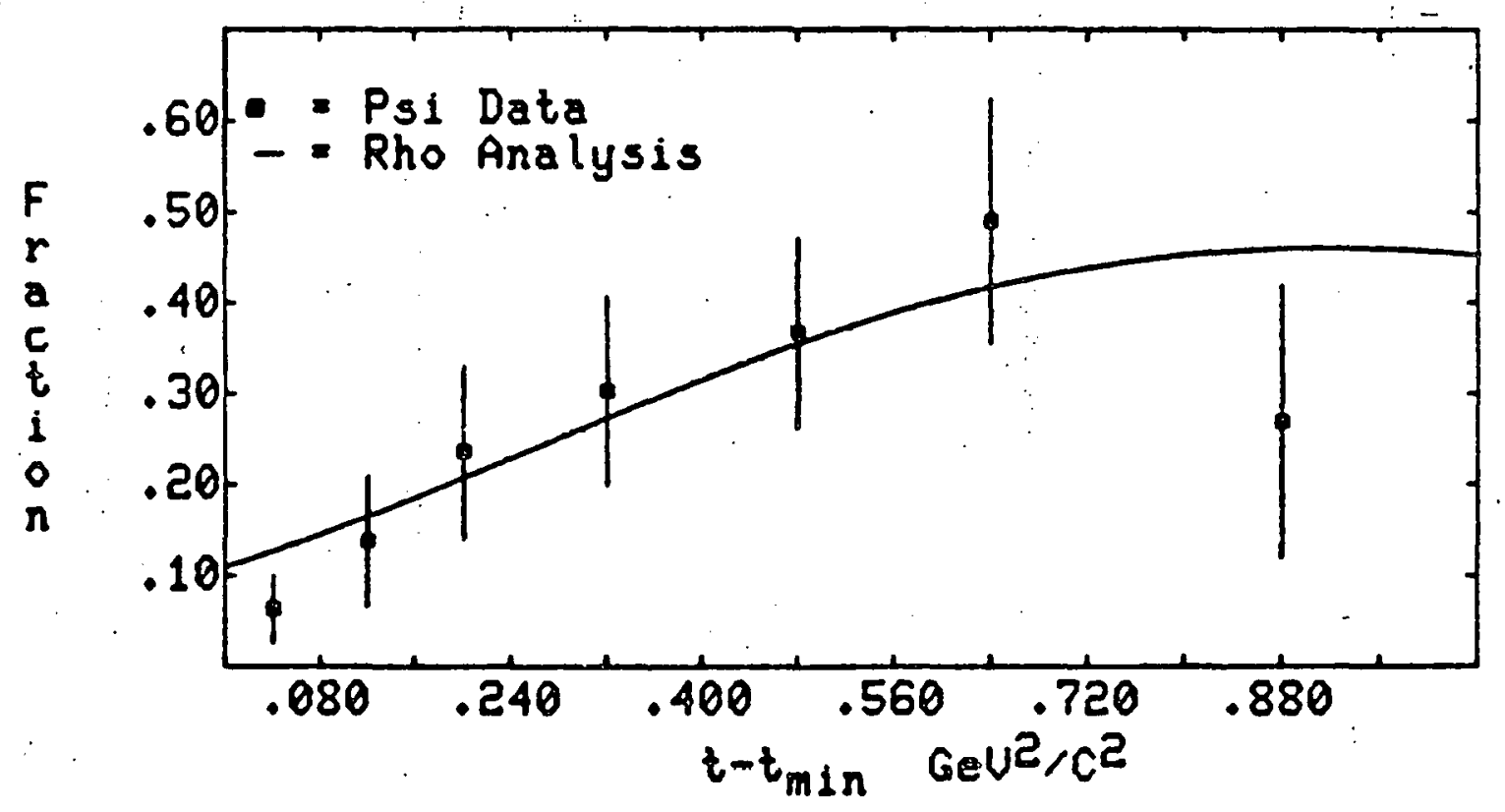

Figure 9.4 
Chapter $\underline{\mathrm{x}}$

Summary and Conclusions

New results from the high energy photoproduction of three vector mesons, the $\rho(770)$, the $\varnothing(1020)$, and the $\psi(3100)$ have been presented. The exclusive $\pi^{+} \pi^{-}$and $\mathrm{K}^{+} \mathrm{K}^{-}$invariant mass distributions have been determined for incident photon energies from $35 \mathrm{GeV}$ to $225 \mathrm{GeV}$ and for 4 -momentum transfers from $t=\emptyset \mathrm{GeV}^{2} / \mathrm{c}^{2}$ to $t=1 \mathrm{GeV}^{2} / \mathrm{c}^{2}$. The fraction of diffractive vector meson photoproduction that is inelastic has been determined as a function of $t$.

Section 1: The Energy Dependence of the elastic $\rho$ and $\phi$ Photoproduction Cross Sections

The elastic $\rho$ and $\varnothing$ photoproduction cross sections obtained are consistent with being independent of energy from $35 \mathrm{GeV}$ to $225 \mathrm{GeV}$ and $165 \mathrm{GeV}$, respectively. The: values determined for the elastic cross sections are:

$$
\begin{aligned}
& \sigma(\gamma+p \rightarrow \rho+p)=10.6+/-\emptyset .1 \text { ub } \\
& \sigma(\gamma+p \rightarrow \varnothing+p)=0.64+/-\emptyset .02 \mathrm{ub}
\end{aligned}
$$

The errors reflect the statistical uncertainty only. We estimate a 158 systematic uncertainty in these results, primarily due to the uncertainty in the calorimeter threshold. The dependence on energy of the elastic $\rho$ cross section (figure 7.11 ) is also consistent with a very slow rise of $19 \%$ from $\sim 10.5$ ub at low energy to $\sim 11.5$ ub at high energy. The depenaence on energy of the elastic $\varnothing$ cross section 
(figure 8.6) is also consistent with a slow rise of 158 from 0.60 ub at low energy to 0.73 ub at high energy.

The diffractive $\psi$ photoproduction cross section (figure 9.1) rises significantly with energy from 20 nb at 60 $\mathrm{GeV}$ to $5 \emptyset \mathrm{nb}$ at $3 \emptyset \emptyset \mathrm{GeV}$. The unambiguous energy dependence of the $\psi$ cross section is markedly different from the energy dependence of the $\rho$ and $\varnothing$ cross sections.

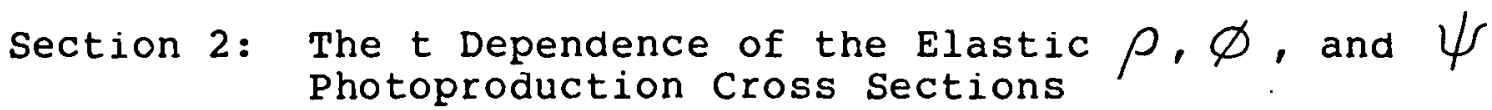

The elastic $t$ distributions for the $\rho, \phi$, and $\psi$ were fit to an exponential function with a quadratic term, $\exp \left(-\mathrm{bt}+c \mathrm{t}^{2}\right)$, from $t=\emptyset \mathrm{GeV}{ }^{2} / \mathrm{c}^{2}$ to $t=1 \mathrm{GeV}{ }^{2} / \mathrm{c}^{2}$. The resulting slope parameters were:

$$
\begin{array}{lll}
\underline{\mathrm{v}} & \underline{\mathrm{b}}(\mathrm{GeV} / \mathrm{c})^{-2} & \underline{\mathrm{c}(\mathrm{GeV} / \mathrm{c})^{-4}} \\
\rho & 8.82+/-.04 & 2.30+/-.08 \\
\varnothing & 7.53+/-.53 & 2.50+/-.73 \\
\psi & 5.55+/-1.18 & 2.85+/-1.32
\end{array}
$$

The errors reflect the statistical uncertainty from the fits only. The parameter $b$ is decreasing with the increasing mass of the scattered vector meson. This decrease in $b$ implies that the interaction radius decreases with increasing mass. The results from section 1 for the $\rho, \varnothing$, and $\psi$. cross sections verify that the interaction radius decreases with the increasing mass of the scattered vector meson. 
Extrapolating the fits to the elastic differential cross sections for the $\rho, \varnothing$, and $\psi$ just described to $t=0 \mathrm{GeV}{ }^{2} / \mathrm{c}^{2}$, we obtain $d \sigma /\left.d t\right|_{t=0}$. The optical theorem and VMD were used to determine the total vector meson-nucleon cross sections as described in chapter 1 :

\begin{tabular}{|c|c|c|c|}
\hline$\underline{V}$ & $\underline{a} \sigma / d t$ & $=\left.\right|_{t=0} \underline{\mathrm{ub}} /\left(\mathrm{GeV} \ddot{V}^{2} / \mathrm{c}^{2}\right)$ & $\sigma_{\text {TOT }}(\mathrm{VN})$ \\
\hline$\rho$ & 89.2 & $+1-$ & $24.7+/-$ \\
\hline$\varnothing$ & 4.06 & $+1-$ & $11.1+/-$ \\
\hline 1 & 0.080 & $+/-0.013$ & $1.6+/-$ \\
\hline
\end{tabular}

The errors reflect the statistical uncertainty only. It is again observed that the heavier particles have a smaller interaction radius. The ratio of the total cross sections for any two. of the vector mesons is approximately equal to the inverse of the ratio of the masses squared:

$$
\begin{array}{lll}
\sigma(\phi N) / \sigma(\rho N)=.45 & : & M_{\rho}^{2} / M_{\varnothing}^{2}=.57 \\
\sigma(\psi N) / \sigma(\rho N)=.065 & : & M_{\rho}^{2} / M_{\psi}^{2}=.063
\end{array}
$$

A comparison of $\sigma(\rho \mathrm{N})$ and $\sigma\left(\phi_{N}\right)$, as determined above, with the predictions of the additive quark model for these cross sections, as described in chapter 1, yields:

$$
\begin{array}{lll}
\sigma(\rho \mathrm{N}) & =24.7 \mathrm{mb}: \mathrm{VMD} \\
{\left[\sigma\left(\pi^{+} \mathrm{P}\right)+\sigma\left(\pi^{-} \mathrm{P}\right)\right] / 2} & =23.7 \mathrm{mb}: \text { AQM } \\
\sigma(\phi \mathrm{N}) & =11.1 \mathrm{mb}: \mathrm{VMD} \\
{\left[\sigma\left(\mathrm{K}^{+} \mathrm{P}\right)+\sigma\left(\mathrm{K}^{-} \mathrm{P}\right)-\sigma\left(\pi^{-} \mathrm{P}\right)\right]} & =15.0 \mathrm{mb},: \mathrm{AQM}
\end{array}
$$


The (KP) and ( $\pi P$ ) total cross sections have been taken from reterence [30]. The prediction of the AQM for $\sigma(\rho N)$ is -in excellent agreement with our result. The AQM prediction for $\sigma(\phi N)$ is 258 greater than our result. The values for $\sigma$ (VN) from E-401 are dependent upon the VMD model and the values of $\gamma_{y}^{2} / 4 \pi$ ueed in the calsulatinn.

Section 3: $\begin{aligned} & \text { Diffractive Inelastic } \rho, \phi ; \text { and } \psi \\ & \text { Photoproduction }\end{aligned}$

The fraction of diffractive $\rho, \phi$, and $\psi$ photoproduction that is inelastic was determined. A fit to the traction of diffractive inelastic production as a function of $t, f(t)$, as obtained from the $\rho$ analysis, was determined to be:

$$
f(t)=\left[1+8.1 \times \exp \left(-4.22 t+2.3 t^{2}\right)\right]^{-1}
$$

The function $f(t)$ was found to describe the diffractive inelastic $\phi$ cross section integrated over $t$, and the fraction of diffractive inelastic $\psi$ production as a function of $t$. We conclude that $f(t)$ is a universal function parametrizing the fraction of diffractive vector meson photoproduction that is inelastic as a function of $t$, regardless of the quark content of the scattered vector meson. This result strongly supports the idea that the contribution from the target nucleon vertex factors from the scattering amplitude illustrated in figure 1.3 . 
Appendix $\underline{A}$

Errors on the Calorimeter Acceptance Measurement

The acceptance, A, is computed by integrating the PAD distribution:

$$
A=\frac{\sum N(x)}{\Sigma N(x) / \varepsilon(x)}
$$

where:

$$
\begin{aligned}
& N(x)=\text { number of events with } P A D=x \\
& \varepsilon(x)=\text { PAD efficiency function }
\end{aligned}
$$

The errors are dominated by contributions at small $\varepsilon(x)$, so we compute the error from the denominator only:

$$
\sigma_{A} \simeq \frac{\Sigma N(x)}{\left[\Sigma \frac{N(x)}{\varepsilon(x)}\right]^{2}} \times \delta\left[\Sigma \frac{N(x)}{\varepsilon(x)}\right]
$$

The error represented by the 2 nd term on the rhs is equal to the sum of the errors in each bin addec in quadrature:

$$
\begin{aligned}
\delta\left[\Sigma \frac{N(x)}{\varepsilon(x)}\right] & =\left[\Sigma \delta\left(\frac{N(x)}{\varepsilon(x)}\right)^{2}\right]^{1 / 2} \\
& =\left[\Sigma \frac{N(x)}{\varepsilon(x)^{2}}\right]^{1 / 2}
\end{aligned}
$$


191

Substituting (A.3) and (A.1) into (A.2), we obtain:

$$
\sigma_{A}=\frac{A \times\left[\Sigma^{N(x)} / \varepsilon(x)^{2}\right]^{1 / 2}}{\sum N(x) / \varepsilon(x)}
$$


Appendix B

Computing the PAD from the Anode ADC's

To compute the PAD off-line, we use the equation:

$$
\operatorname{PAD}_{S}=\sum_{i=1}^{21} \mathbf{x}_{i} / R_{i}
$$

where

$$
\begin{aligned}
\mathrm{PAD}_{\mathrm{S}} & =\text { software } \mathrm{PAD} \\
\mathrm{x}_{\mathrm{i}} & =\text { anode for } H \mathrm{HC}_{i} \\
\mathrm{R}_{i} & =\text { anode/dynode ratio for } \mathrm{HC}_{i}
\end{aligned}
$$

The anode/dynode ratios, $R$, were measured by an iterative procedure. The first pass $R$ values, $R_{0}$, were obtained by scanning the 21 . HC modules for the module, with the largest anode signal. If this anode signal represented $>80 \%$ of the total pulse height in the calorimeter, the hardware dynode sum was assumed to be the dynode signal associated with this large anode:

$$
\begin{aligned}
R_{0} & =\frac{x(>80 \%)}{P A D_{H}} \\
\text { where } \mathrm{PAD}_{H} & =\text { hardware } \mathrm{PAD}
\end{aligned}
$$

The 2nd pass values, $R_{1}$, were obtained by iteration using the first pass valies and equation (R.1):

$$
R_{1}=\frac{x^{k}}{\left[P A D_{H}-\sum_{i \neq k}^{21} x_{i} / R_{0}^{i}\right]}
$$

After a few iterations, R stabilized (figure B.1). 


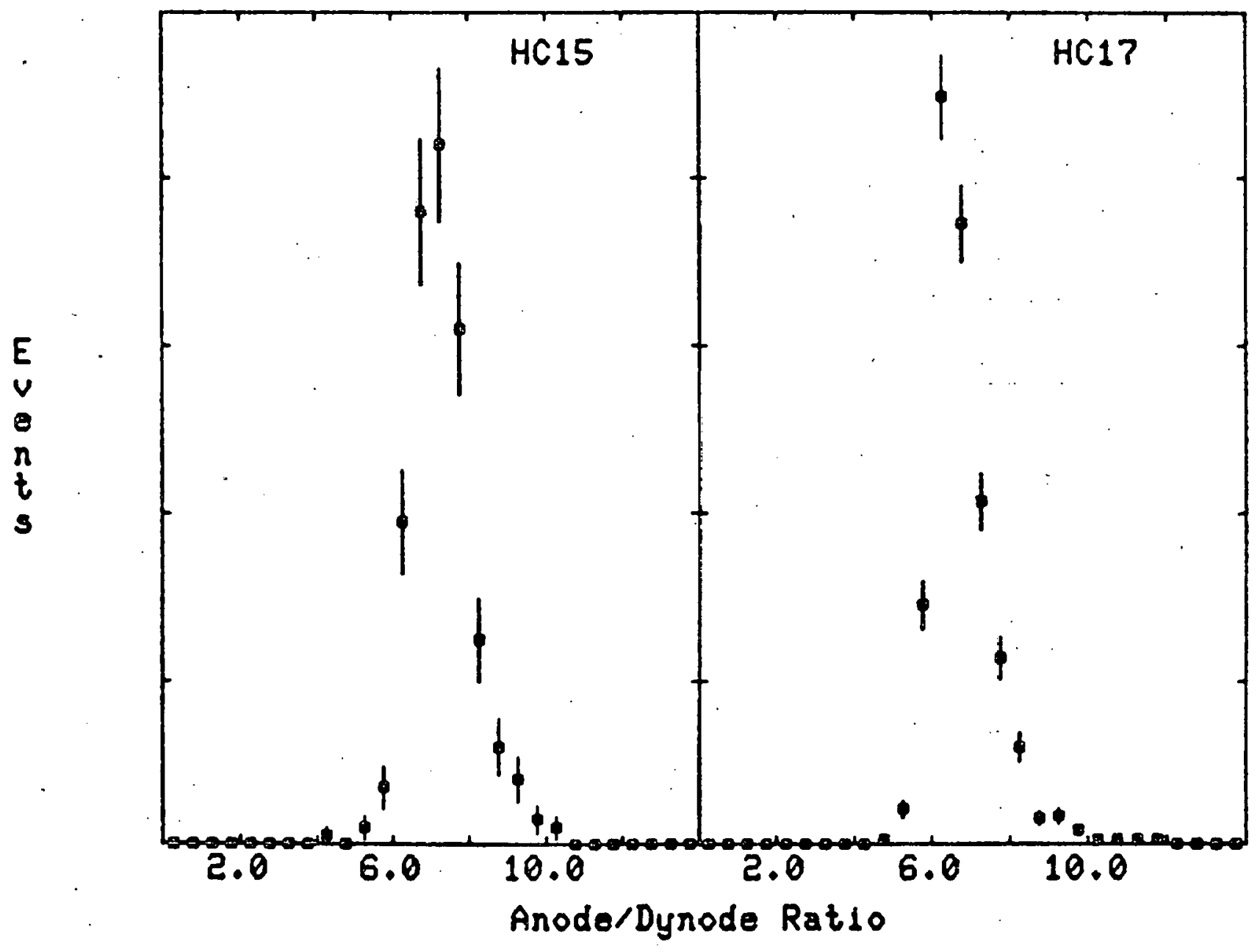


194

Appendix $\underline{C}$

Corrections to the cross section

Section 1: Absorption of $\pi, K$ in the spectrometer

For a set of $\mathrm{N}$ particles, the probability for $\mathrm{n}$. of them being absorbed within a distance $x$ is given by the binomial distribution:

$$
P(n, x)=\frac{N !}{n !(N-n) !} \cdot P(x)^{n} \cdot q(x)^{(N-n)} ; \quad P+q=1
$$

For $\rho \rightarrow \pi^{+} \pi^{-}$or $\varnothing \rightarrow \mathrm{K}^{+} \mathrm{K}^{-}, \mathrm{N}=2$. The probability that at least one particle has been absorbed is:

$$
\mathrm{P}_{A B S}=\mathrm{P}(1)+\mathrm{P}(2)=2 \mathrm{Pq}+\mathrm{P}^{2}
$$

The differential probability for absorption of a particle with an absorption length $\lambda$ in an amount of material $x$ is:

$$
\frac{d P}{d x}(x, \lambda)=\frac{1}{\lambda} e^{-x / \lambda}
$$

Therefore

$$
\begin{aligned}
& P(x)=\int_{0}^{-x} \frac{d P\left(x^{\prime}\right)}{d x^{\prime}} d x^{\prime}=1-e^{-x / \lambda}(c .3) \\
& q(x)=1-P(x)=e^{-x / \lambda}
\end{aligned}
$$


Substituting (C.3) and (C.4) into (C.1) obtains:

$$
P_{A B S}(x, \lambda)=1-e^{-2 x / \lambda} \quad(c .5)
$$

To compute the amount of material in the spectrometer seen by pions and kaons, we take the absorption length $\lambda$ from the particle properties book [8] for protons and scale it by the ratio, $R$, of the $\pi / P$ and $K / P$ cross sections. Thus, $\lambda \rightarrow R \lambda$, where:

$$
\begin{aligned}
& R_{\pi}=\sigma_{p} / \sigma_{\pi}=\frac{39 \mathrm{mb}}{24 \mathrm{mb}}=1.625 \\
& -R_{k}=\frac{\sigma_{p}}{\sigma_{k}}=\frac{39 \mathrm{mb}}{19 \mathrm{mb}}=2.053
\end{aligned}
$$

Material

$\mathrm{LH}_{2}$ in target

T-counter scintillator

Air (target to $\mathrm{HxV}$ ) $\underline{x(c m)}$

$$
\text { (cm) } \underline{P}_{\pi}
$$

$\underline{\underline{P}}_{K}$

$$
20.32
$$

790

.9 .31

.025

.508

68.5

.0091

$.00 / 2$

$127 \emptyset$

$67500 \quad .023$

Total 
Section 2: Decay of $\pi, k$ in spectrometer

The differential probability for a particle of mass $M$, proper lifetime $\mathcal{T}$, and momentum $P$ to decay at a distance $x$ is:

$$
\frac{d P}{d x}(x, P)=\frac{M}{P C T} \times e^{-M x / P C T}
$$

Using the arguments of section 1, we obtain:

$$
P_{D E C A Y}(X, P)=1-e^{-2 M X / P_{C} T}
$$

In the C.M. frame, the decay angle distribution for a spin 1 object decaying into 2 spin $\emptyset$ objects is proportional to $\sin ^{\pi} \theta \mathrm{cm}$. Consequently the $\pi / K$ pairs tend to be symmetric in cnergy in the laboratory and we use $\mathrm{p}_{\pi, k}=\mathrm{F}_{\beta, \infty} / 2$ :

$$
P_{D E C A Y}(X, E)=1-e^{-4 M x / E C T}
$$

There is some ambiguity as to what decay length to use. We choose the distance from the target center to the bend plane in M2 as the decay length. This results in $x=260 "$. See table 8.1 for examples of $1-P_{D E C A Y}(x, E)$. 
Section 3: Photon Conversion and Target "Self-Shielding"

If a photon in the primary beam converts to an $e^{+} e^{-}$pair upstream of M2, this pair will be swept away from the quantameter resulting in a systematically low measurement of the total beam power. A correction is made based on the amount of material, in: radiation lengths, between the upstream end of the target and M2. The photon energy loss due to pair conversion is given by

$$
I=I_{0} e^{-\lambda x / L}
$$

where

$$
\begin{aligned}
& I=\text { photon flux } \\
& \lambda=.773 \text { independent of absorber } \\
& \mathrm{X}=\text { length of material } \\
& L=1 \text { radiation length for material } \mathrm{x}
\end{aligned}
$$

material

Upstream mylar

target window

$\mathrm{LH}_{2}$ target

Downstream mylar

target window

T-counters

MWPC chamber

windows

Air $\left(-253^{\prime \prime} \rightarrow-36^{\prime \prime}\right)$

TOTAL $x(\mathrm{rm}) \quad \mathrm{L}(\mathrm{cm}) \quad(\mathrm{X} / \mathrm{L})$

$.01905 \quad 28.7 \quad .009664$

$40.64 \quad 89 \emptyset$

.0457

$.0254 \quad .28 .7 \quad .000885$

$\begin{array}{lll}.508 & 42.9 & .0118\end{array}$

.002

55130050

.0183 
The photon flux incident on the downstream target nucleons is less than the flux incident on the upstream target nucleons due to e e pair conversion. This is called "self shielding". The correction is the integral of equation (C.6):

$$
\begin{aligned}
I= & I_{0}\left(\frac{\lambda x}{L}\right)\left(1-e^{-\lambda x / L}\right)^{-1} \\
x / L & =\text { upstream target window }+L_{2} \\
& =.000664+.0457=.0464
\end{aligned}
$$

Putting together these two corrections we obtain for the true quantameter charge, $Q_{T}$ '

$$
\begin{aligned}
& Q=Q_{O B S} \times \text { pair conversion } \times \text { self shielding } \\
& Q_{T}=Q_{O B S} \times e^{(.773)(.0794)} \times \frac{\left(1-e^{-(.773)(.0464)}\right)}{(.773)(.0464)} \\
& Q_{T}=Q_{\text {OBS }} \times(1.963) \times(.982) \\
& Q_{T}=1.044 \times Q_{O B S} .
\end{aligned}
$$


Section 4: 2-Track MWPC Reconstruction Efficiency

The average efficiency per plane of the 15 MWPC wire planes was $\varepsilon_{P L}=.983+/-.0095$. The track reconstruction algorithm required at least 14 planes to reconstruct a : 5-chamber track. The efficiency for reconstructing a 5 -chamber track, $\varepsilon_{T K}$, is therefore given by:

$$
\varepsilon_{T k}=(.983)^{15}+\frac{15 !}{14 ! 1 !}(.983)^{14}(.017)^{1}=.974
$$

The efficiency for reconstructing an event with 2 -chamber tracks is therefore:

$$
\left(\varepsilon_{T K}\right)^{2}=.948
$$

Section 5: AW Counter Accidental Rate

The AW counters were used as a software veto. The accidental veto rate was measured using $P$ in 5 events within $+/-5 \emptyset \mathrm{MeV}$ of the $\rho$ mass. The recoil proton was required to be an unambiguously identified proton coplanar to the $\rho$ in at least the first two recoil detector rings. This ensured a sample of exclusive 2-track final states. The AW accidental rate was determined to be .083 giving a veto efficiency of .917 . 
Section 6: Other Corrections

$\begin{array}{ll}\text { T-counter efficiency } & =.99 \\ >20 \text { hits/plane in MWPC } & =.99 \\ \text { too many hits in PØ } & =.995 \\ \text { extra track from e e } & =.985 \\ \text { pair ( } 2 \text { photon bucket) } & \\ \text { AØ veto effiency } & =.985\end{array}$




\section{Appendix $\underline{D}$}

Classification of Events by the Recoil Detector Analysis

An event will be analyzed and classified as one of 16 different event types corresponding to different event topologies. Numbered as case -2 through case 13 these 16 categories all belong to one of four different group types:

1) Positive Elastic

These are events where $t$ is large enough to propel a recoil proton through the appararatus and all coplanarity and pulse height requirements are satisfied.

2) Consistent with Elastic

These are events where $t$ is small enough to trap the recoil proton in the target materials. No other evidence of inelasticity in the recoil detector is seen (too many tracks, hits, etc.).

3) Inelastic

These are events with many extra tracks and/or hits in the recoil detector. Also included are events with one track in the recoil detector that is inconsistent with coplanarity beyond resolution tolerances.

4) Not Accepted

Very low $t$ events in the upstream end of the target and very high $t$ events $\left(t>2.5 \mathrm{GeV}^{2} / \mathrm{c}^{2}\right)$ in the downstream end of the target will not enter the fiducial volume of the recoil detector.

The specific criteria for each of the 16 catagories are described below. 
Case -2: Events where z-predicted at the inner $r$ ing is within $1.2 * \sigma_{z}$ of being upstream of the detector and the recoil counter is off

Case -1: Predict proton range-out in target to within $2 * \sigma_{t}$ and there are $\leq 2$ unassociated hits in the recoil counter; if $\left(t+2 \sigma_{t}\right)$ would propel the proton out of the target, the event is reanalyzed on that basis

Case 0: Predict proton range-out in target to within $2 * \sigma_{t}$ and there are 1 unassociated hits in the recoil counter; if $\left(t+2 \sigma_{t}\right)$ would propel the proton out of the target, the event is reanalyzed on that basis

Case 1: Best elastic information; 2-ring matched track, positive proton pulse height in at least one ring, no unassociated hits

Case 2: Good elastic information; case\#l with 1 extra hit; 2-ring matched track without positive proton pulse height and no extra hits; l-ring matched track with positive proton pulse height and no extra hits

Case 3: Good elastic information; case \#l with 2 extra hits; case \#2 with 1 extra hit; 1-ring matched track without positive proton pulse height and no extra hits

Case 4: Sloppy elastic events; case \#l with $>2$ extra hits; case $\# 2$ with $\geq 2$ extra hits; case \#3 with 1 extra hit

Case 5: Predict 1-ring track and the recoil counter is off; the predicted hit is accepted in $z$ by at least $1.2 * \sigma_{t}$; if $\left(t-2 \sigma_{t}\right)$ is consistent with proton range-out, this gets changed to case $\emptyset$

Case 6: Predict 2-ring track and the recoil counter is off; the predicted track is accepted in $z$ by at least $1.2 * \sigma_{z}$; if $\left(t-2 \sigma_{t}\right)$ is consistent with proton range-out, this gets changed to case $\emptyset$

Cases 7\&8: Inelastic; $1 \& 2$ ring tracks with no positive proton pulse height and $\geq 3$ extra hits; also no matched tracks and $\geq 2$ extra hits 
Case 9: Inelastic; $1 \& 2$ ring matched tracks with positive proton pulse height and 1 extra track and possibly other unassociated hits in detector

Case 10: . Inelastic; case \#9 without positive proton pulse height

Case 11: Most inelastic; case \#9 and case \#10 with extra tracks 22 ; also no matched tracks with extra tracks $\geq 1$ and possibly other unassociated hits

Case 12: Predict proton range-out in target and $\geq 3$ extra hits or 1 extra tracks; if $\left(t+2 \sigma_{t}\right)$ would escape target material, event is re-analyzed under that assumption

Case 13: Events where z-predicted at the inner $r$ ing is within $1.2 \sigma_{z}$ of being downstream of the detector and the recoil counter is off; this occurs only for events with $t \gtrsim 2.5 \mathrm{GeV}^{2} / \mathrm{c}^{2}$

A study was done to determine into which group the somewhat ambiguous cases 4,5 and 6 should be placed. Pin 5 events with a di-pion mass within $+/-200 \mathrm{MeV}$ of the $\rho$ mass were chosen. A tight target cut $\left(-262 "<z<-255.5^{\prime \prime}\right)$ was made to ensure acceptance into the recoil detector fiducial volume. If either track was identified as a kaon by the Cerenkov system the event was cut. A $t$-cut of $t>15 \mathrm{GeV}^{2} / \mathrm{c}^{2}$ was made to ensure that the proton was energetic enough to exit the Larget materials.

The data were divided into three groups. Cases 1,2 and 3 were called elastic, cases 7 to 11 were called inelastic, and cases 4,5 and 6 were called ambiguous. Figure D.l shows the $t$ distribution measured for each group. 
The unambiguous result is that cases 4,5 and 6 are extremely "inelastic-like", closely resembling cases 7 to 11 .

The final condensation of cases used in this analysis was:
1) Elastic: cases $-1, \emptyset, 1,2,3$
2) Inelastic: cäses $4,5,6,7,8,9,10,11,12$
3) Not Accepted: cases $-2,13$ 


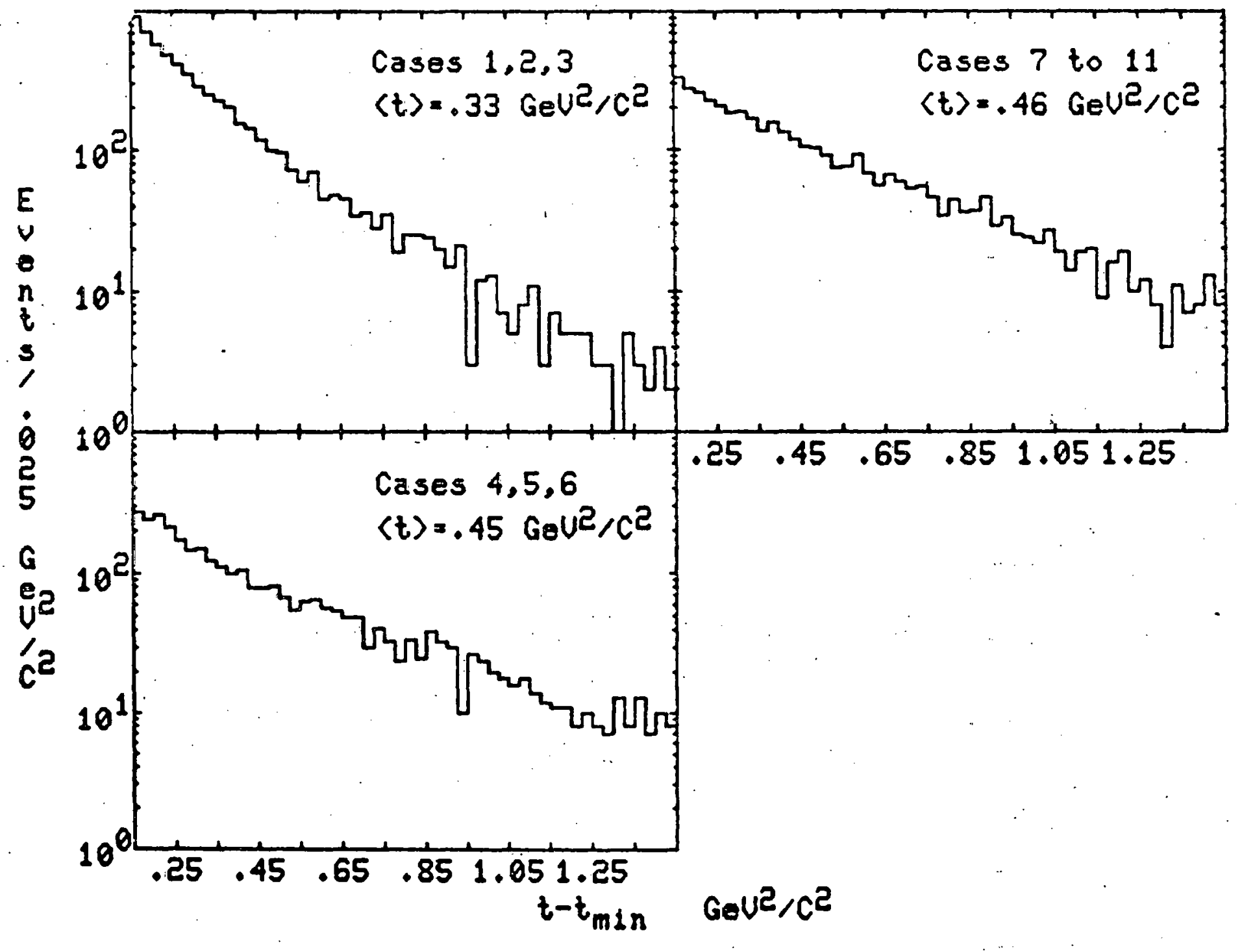

Figure D.1 


\section{Appendix E \\ $\underline{K}_{L}^{\circ}$ Scale Factor}

The $K_{L}^{0}$ backround is proportional to the numbers of protons on target, which was measured by a secondary emission monitor (SEM). The $K_{L}^{0}$ runs were recorded with 6 radiation lengths of lead in the beam. Therefore, a correction for $K_{L}$ absorption in the lead must be made: For the scale factor, f, we obtain:

$$
f=\frac{(L T * S E M)_{\gamma}}{(L T * S E M)_{K_{L}}} e^{x / L}
$$

where

$$
\begin{aligned}
L T \star S E M & =\begin{array}{l}
\text { Electronic livetime } \mathrm{x} \text { number of } \\
\text { protons on target }
\end{array} \\
\mathrm{L}= & \text { Kaon absorption length in lead } \\
\mathrm{x}= & \begin{array}{l}
\text { Nuclear absorption length of } 6 \\
\text { radiation lengths of lead }
\end{array}
\end{aligned}
$$

thus:

$$
\begin{aligned}
f & =\frac{.762 \times 1.077 \times 10^{16}}{899 \times 4.9011} e^{3.36 / 18.5} \\
& =2.234
\end{aligned}
$$




\section{References}

1. H.R. Crouch et al, Phys. Rev. Lett. 13, 640(1964)

2. R. Spital and D.R. Yennie, Nucl. Phys. B106, 269(1976)

3. J.J. Sakurai, Ann. Phys. 11, 1(1960)

4. M. Gell-Mann and F. Zachariasen, Phys. Rev. 124, 953(1961)

5. M. Gell-Mann, D. Sharp,"'and W.O. Wagner, Phys. Rev. Lett. 8, 261 (1962)

6. M. Ross and L. Stodolsky, Phys. Rev. 149, 1172(1966)

7. H. Joos, Phys. Lett. B24. $103(1967)$

8. B. Knapp et al, Phys. Rev. Lett. $37,882(1976)$

9. D.W.G.S. Leith, in Electromagnetic Interactions of Hadrons, edited by A. Donnachie and G. Shaw

(Plenum, New York), Vol. I, 345(1977)

10. G.J. Gounaris and J.J. Sakurai, Phys. Rev. Lett. 21, 244 (1968)

11. H.J. Lipkin, Phys. Rev. Lett. 16, 1015(1966)

12. M. Binkley at al, Phys. Rev. Lett. 48, 73(1982)

13. D.o. Caldwell et al, Phys. Rev. Lett. 40, 1222 (1978)

14. M.C. Goodman, Ph.D. thesis, University of Illinois, 1979

15. M.S. Atiya, Ph.D. thesis, Columbia University, 1984

16. C.J. Olszewski, private communication, University of Illinols

17. R.M. Egloff, Ph.D. thesis, University of Toronto, 1979

18. J.M. Bronstein, Ph.D. thesis, University of Illinois, 1977

19. P. "Chico" Avery, Ph.D. thesis, University of Illinois, 1980

20. J.J. Russell, Ph.D. thesis, University of Illinois, 198v

21. H.J. Behrend, Nucl. Phys. Bl44, 22(1978)

22. P. Soding and A. Krass, Phys. Lett. 19. 702(1965) 
208

23. J.D. Jackson, Nuavo Cimento, 34, 1644(1964)

24. C. Berger et al, Phys. Lett. B39, 659(1972)

25. Particle Data Group, Rev. Mod. Phys. 48, no. 2, Part II (Apri1, 1976)

26. A.R. Clark at al, Phys. Rev. Lett. 43, 187(1979)

27. T. Nash at al, Phys. Rev. Lett. 36, 1233(1976)

28. U. Camerini at el, Phys." Rev. Lett. 35,483(1975)

29. B. Gittleman at al, Phys, Rev. Lett. 35, 1616(1975)

30. A.s. Carroll et al, Phys. Rev. Lett. 33, $932(1974)$ 